\title{
Mesoionic Carbenes in Low- to High-Valent Vanadium Chemistry
}

Florian R. Neururer, ${ }^{a}$ Shenyu Liu, ${ }^{b}$ Daniel Leitner, ${ }^{a}$ Marc Baltrun, ${ }^{b}$ Katherine R. Fisher, ${ }^{c}$ Holger Kopacka, ${ }^{a}$ Klaus Wurst, ${ }^{a}$ Lena J. Daumann, ${ }^{\mathrm{c}}$ Dominik Munz ${ }^{* \mathrm{~d}}$ and Stephan Hohloch*a .

${ }^{a}$ University of Innsbruck, Institute of Inorganic, General and Theoretical Chemistry, Innrain 80-82, 6020 Innsbruck, Austria, E-Mail: Stephan.Hohloch@uibk.ac.at

${ }^{\mathrm{b}}$ University of Paderborn, Faculty of Science, Department of Chemistry, Warburger Straße 100, 33098 Paderborn, Germany

${ }^{c}$ Ludwigs-Maximilians-University Munich, Department Chemie, Butenandtstraße 5-13 Haus D, 81377 Munich, Germany

d Saarland University, Fakultät NT, Inorganic Chemistry: Coordination Chemistry, Campus C4.1, 66123 Saarbrücken, Germany, E-Mail: Dominik.Munz@uni-saarland.de

Supporting Information

\section{Table of contents}

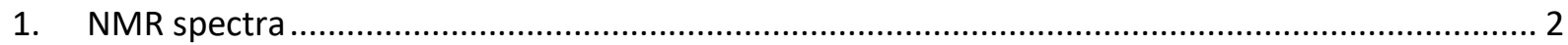

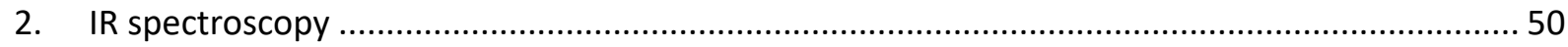

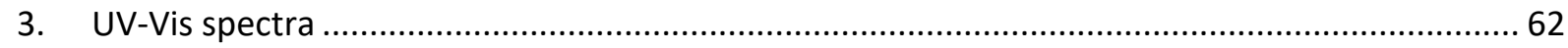

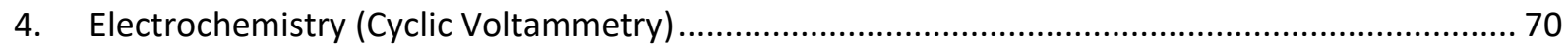

5. Quantum Chemical Calculations ……................................................................................. 72

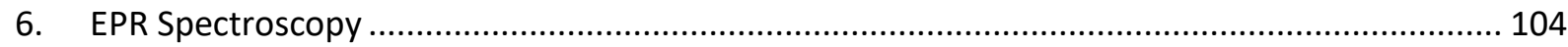

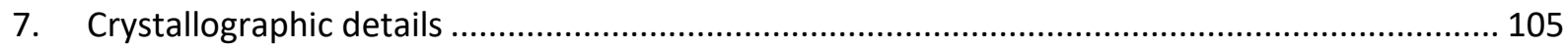

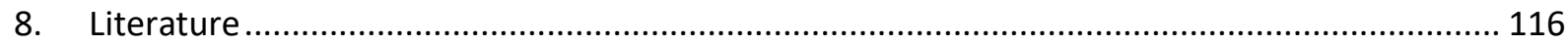




\section{NMR spectra}

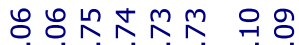

NNN No

$\stackrel{\text { mi }}{\text { mi }}$

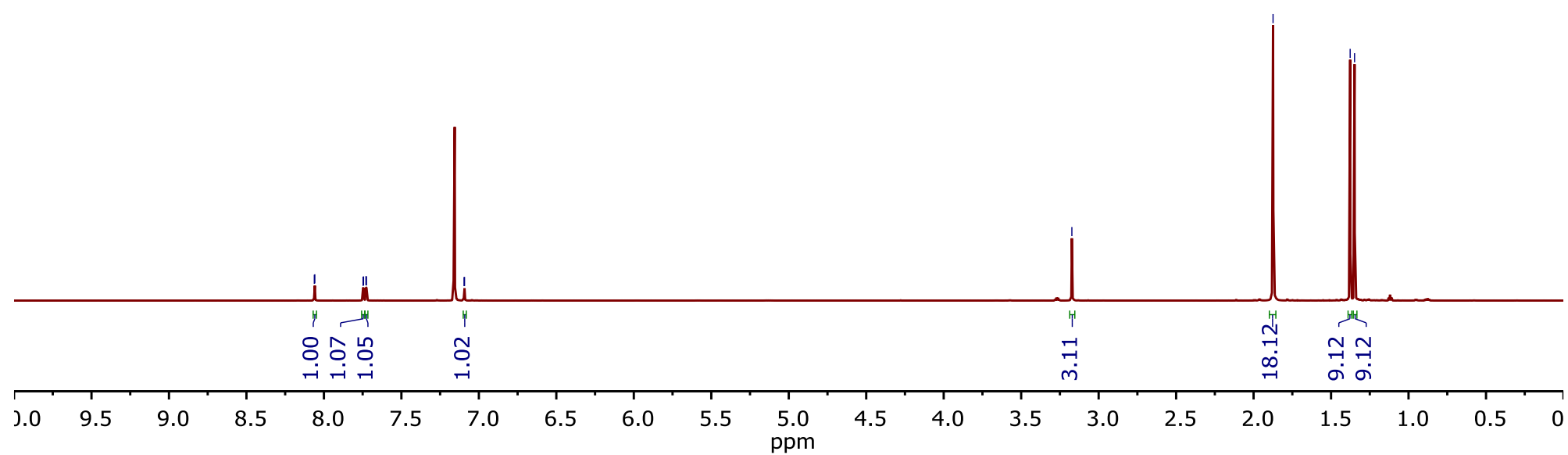

Figure S 1: $\quad{ }^{1} \mathrm{H}$ NMR of 1 in $\mathrm{C}_{6} \mathrm{D}_{6}$ at $298 \mathrm{~K}$.

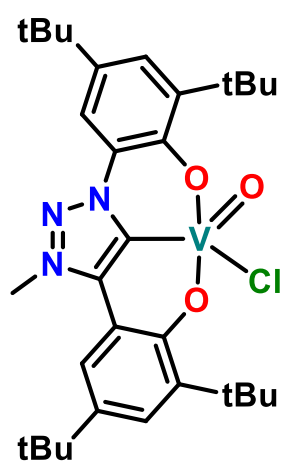



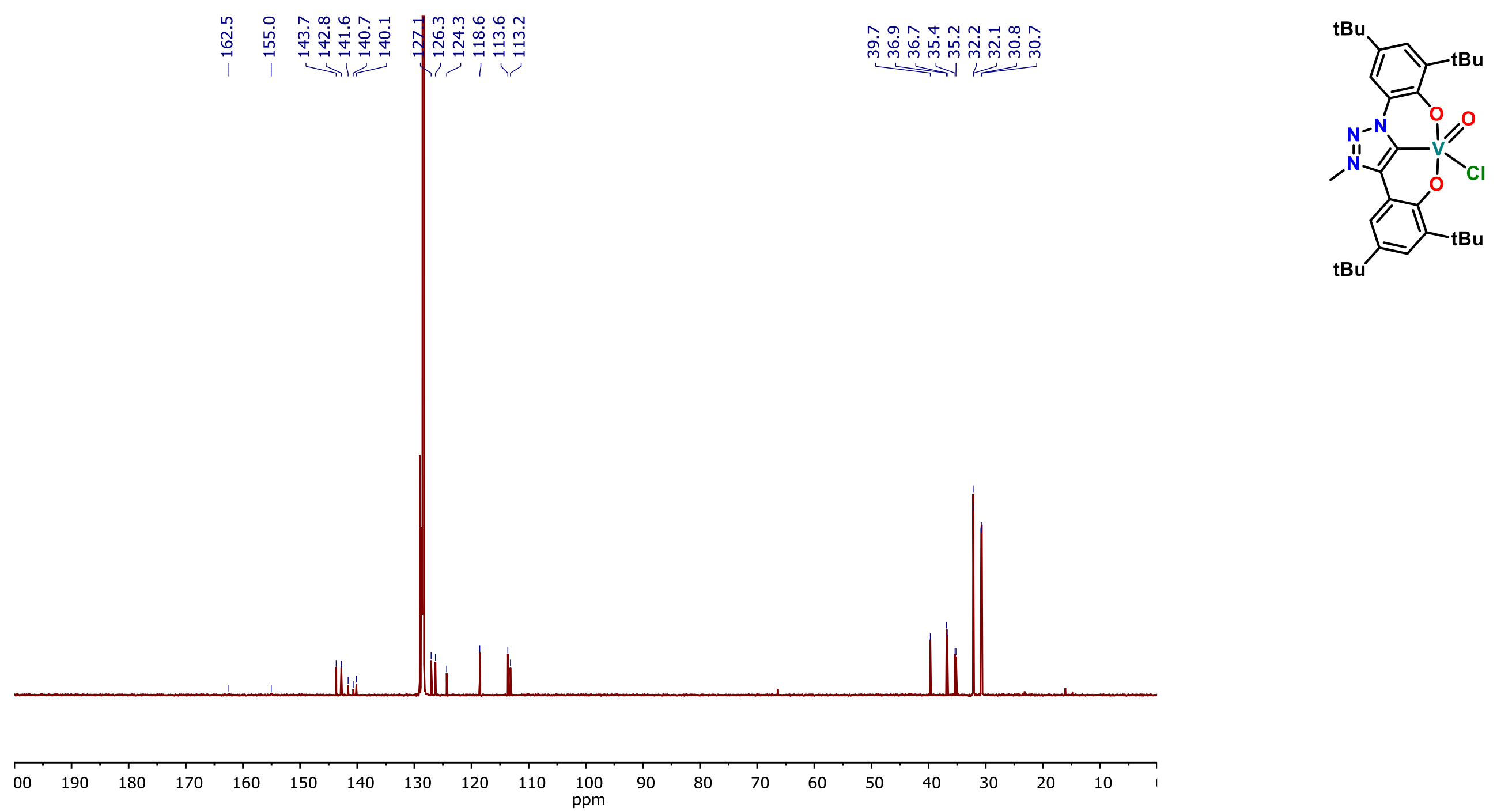

Figure S 2: $\quad{ }^{13} \mathrm{C}$ NMR of $\mathbf{1}$ in $\mathrm{C}_{6} \mathrm{D}_{6}$ at $298 \mathrm{~K}$. 

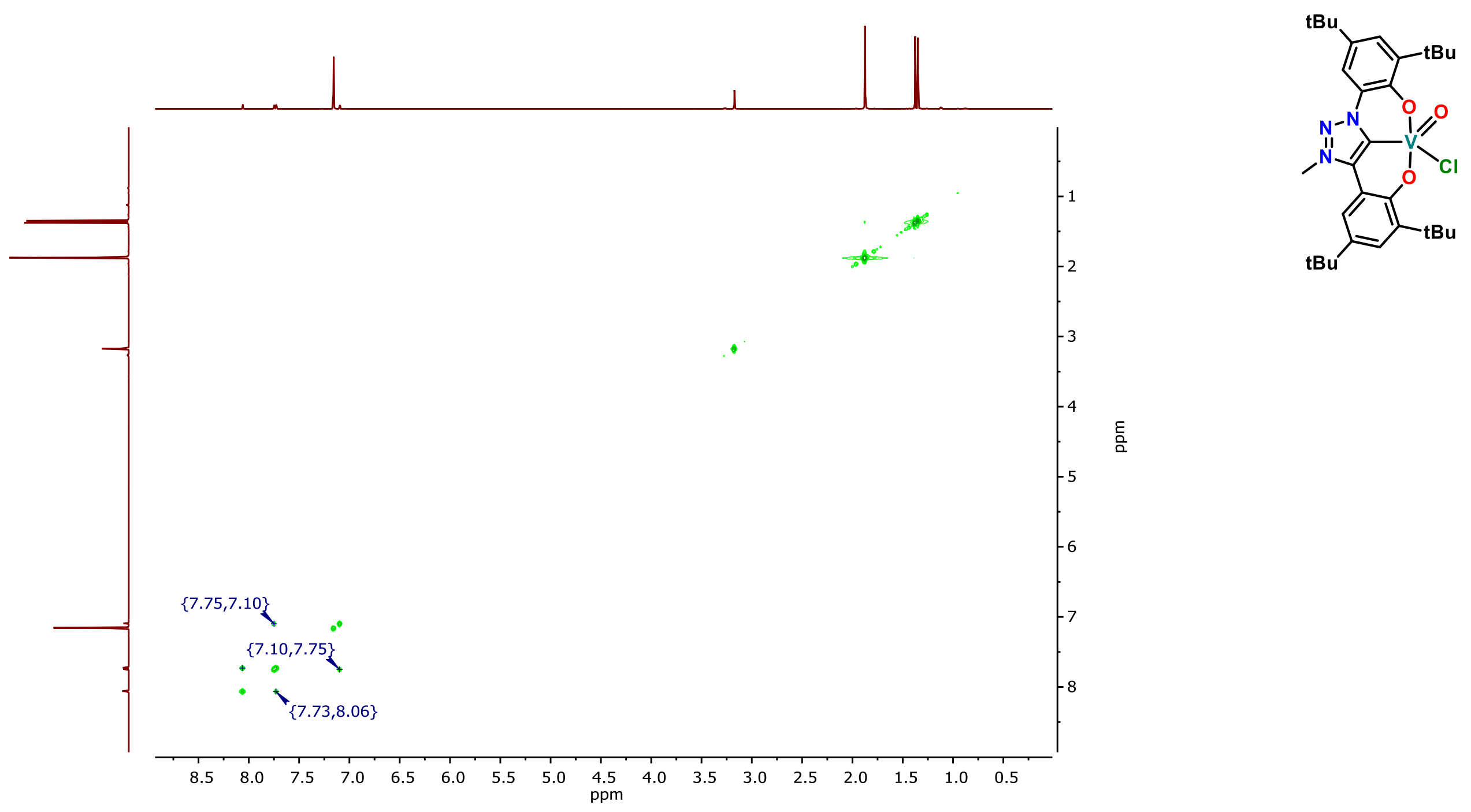

Figure S 3: $\quad{ }^{1} \mathrm{H}-{ }^{1} \mathrm{H}$ COSY of 1 in $\mathrm{C}_{6} \mathrm{D}_{6}$ at $298 \mathrm{~K}$. 

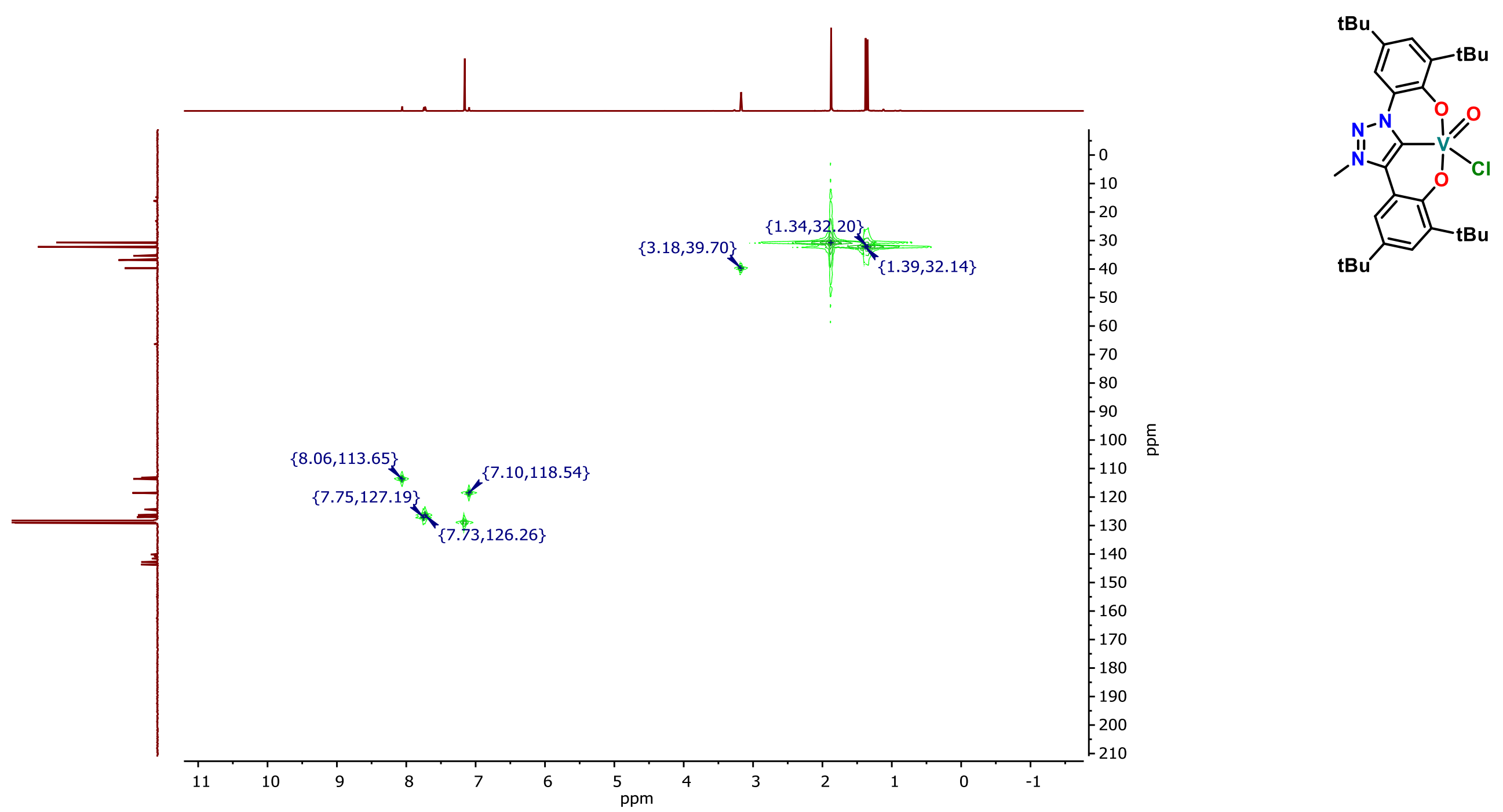

Figure S 4: $\quad{ }^{1} \mathrm{H}_{-}{ }^{13} \mathrm{C}$ HSQC of $\mathbf{1}$ in $\mathrm{C}_{6} \mathrm{D}_{6}$ at $298 \mathrm{~K}$. 

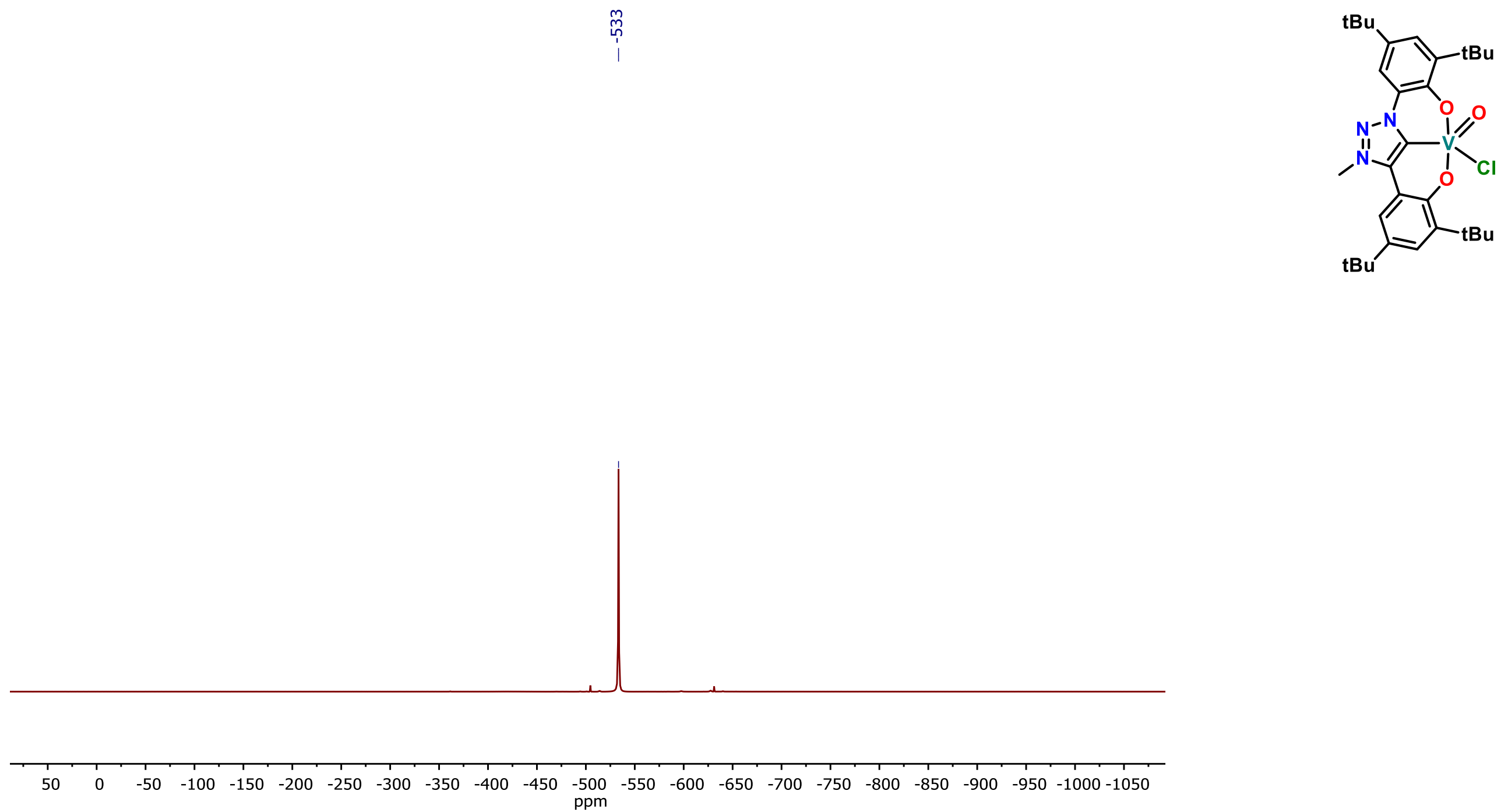

Figure S 5: $\quad{ }^{51} \mathrm{~V}$ NMR of $\mathbf{1}$ in $\mathrm{C}_{6} \mathrm{D}_{6}$ at $298 \mathrm{~K}$. 

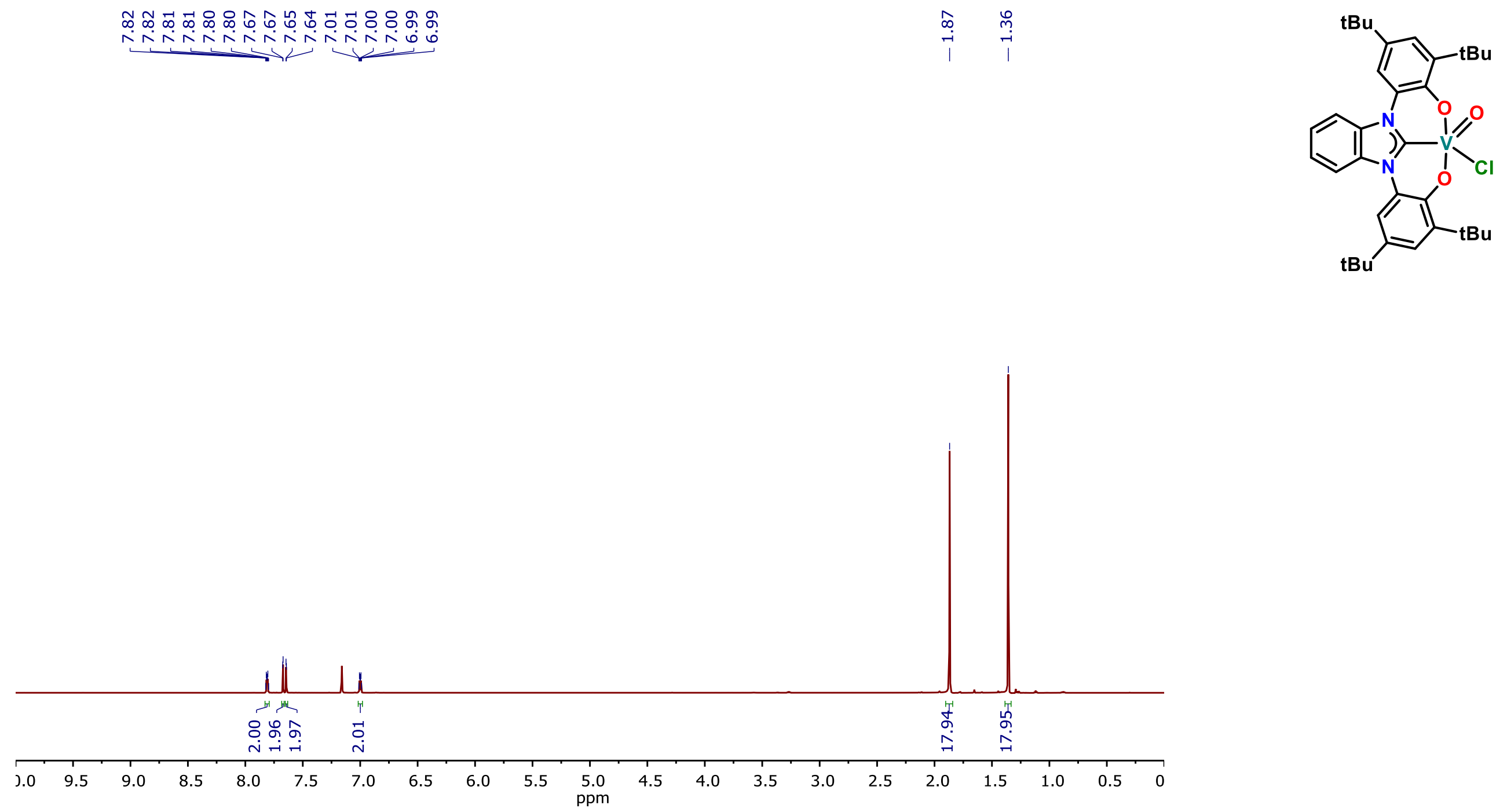

Figure S 6: $\quad{ }^{1} H$ NMR of 2 in $C_{6} D_{6}$ at $298 \mathrm{~K}$. 

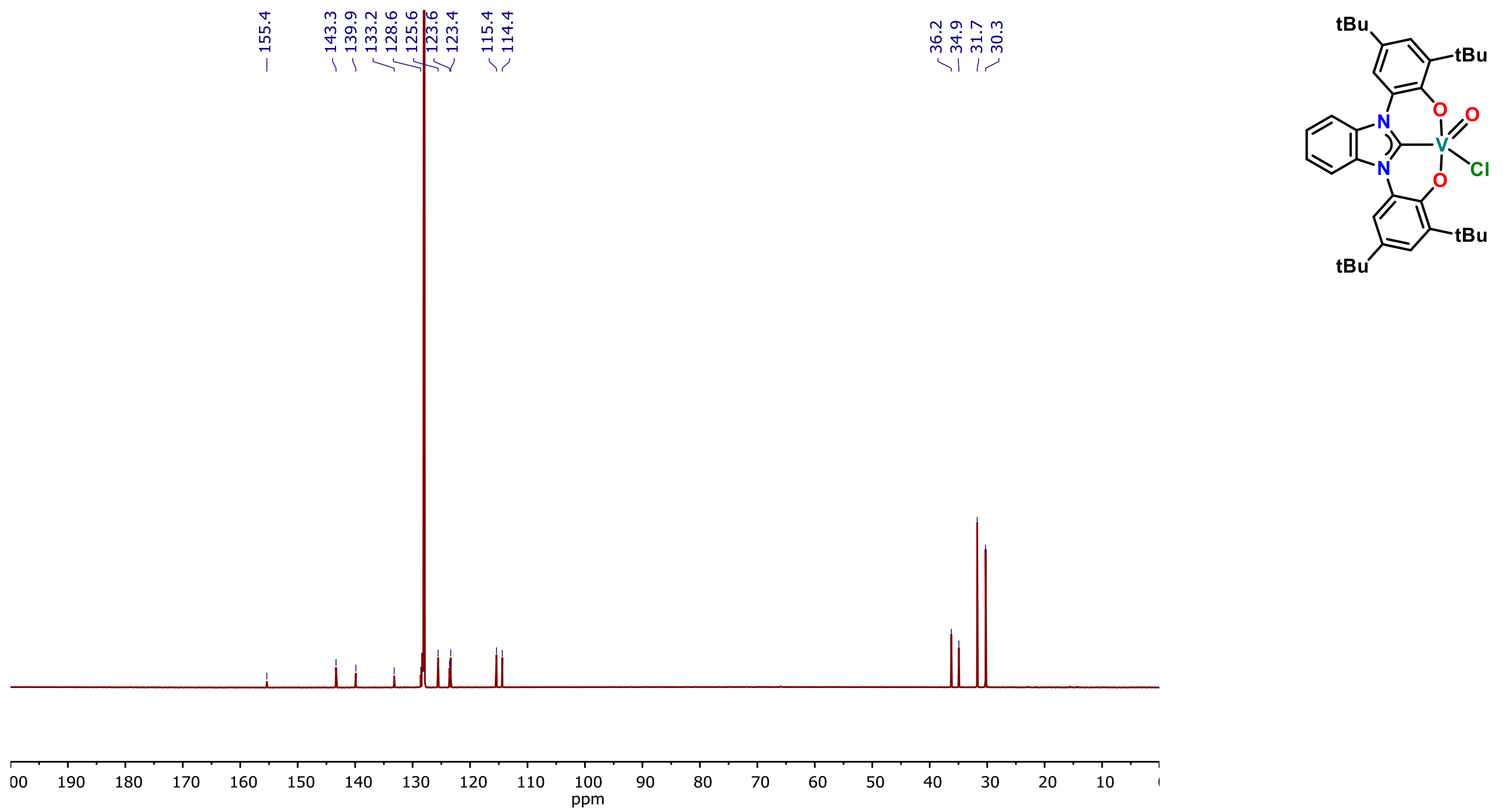

Figure S 7: $\quad{ }^{13} \mathrm{C}$ NMR of 2 in $\mathrm{C}_{6} \mathrm{D}_{6}$ at $298 \mathrm{~K}$. 

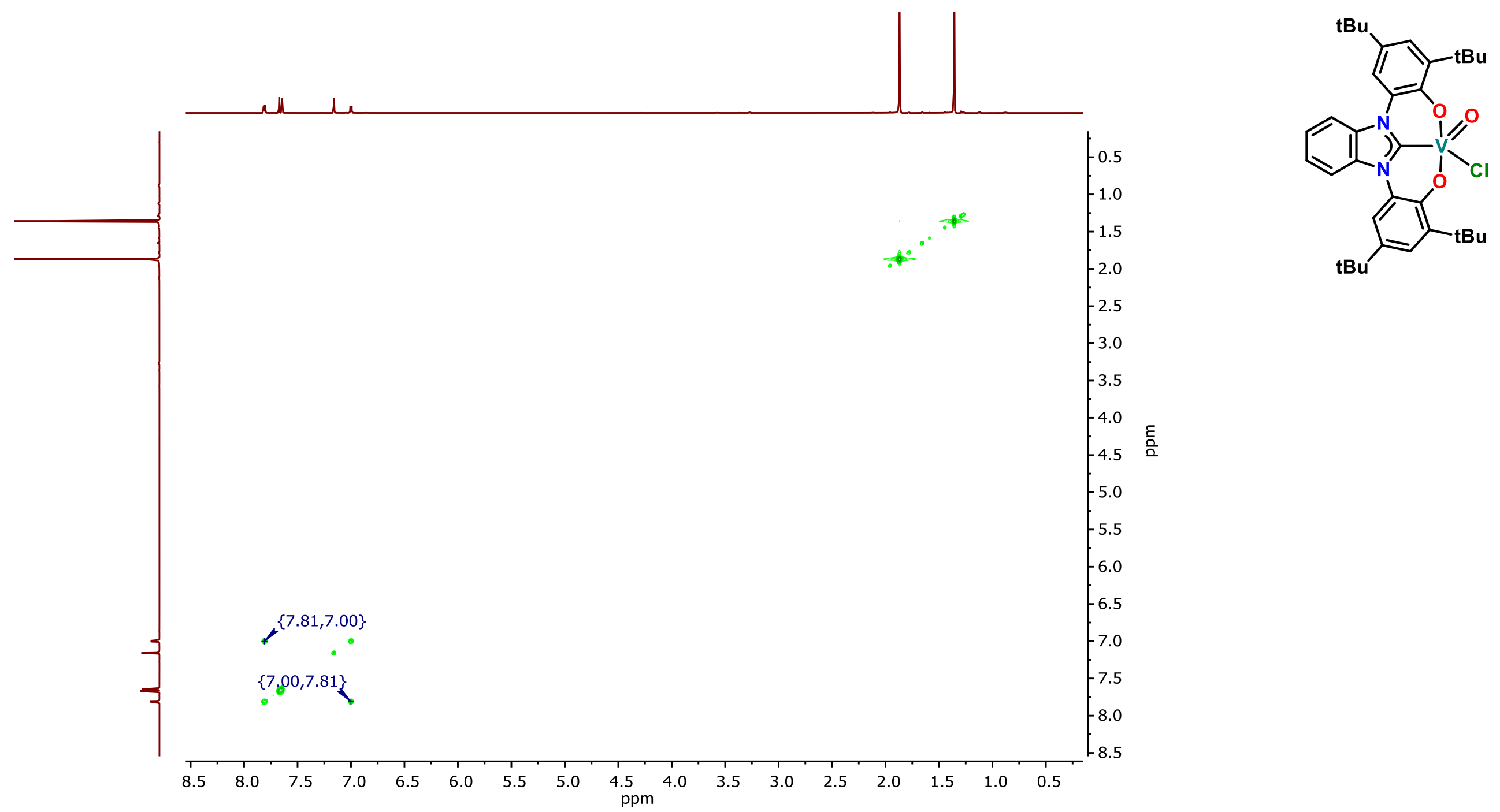

Figure S 8: $\quad{ }^{1} \mathrm{H}-{ }^{1} \mathrm{H}$ COSY of 2 in $\mathrm{C}_{6} \mathrm{D}_{6}$ at $298 \mathrm{~K}$. 

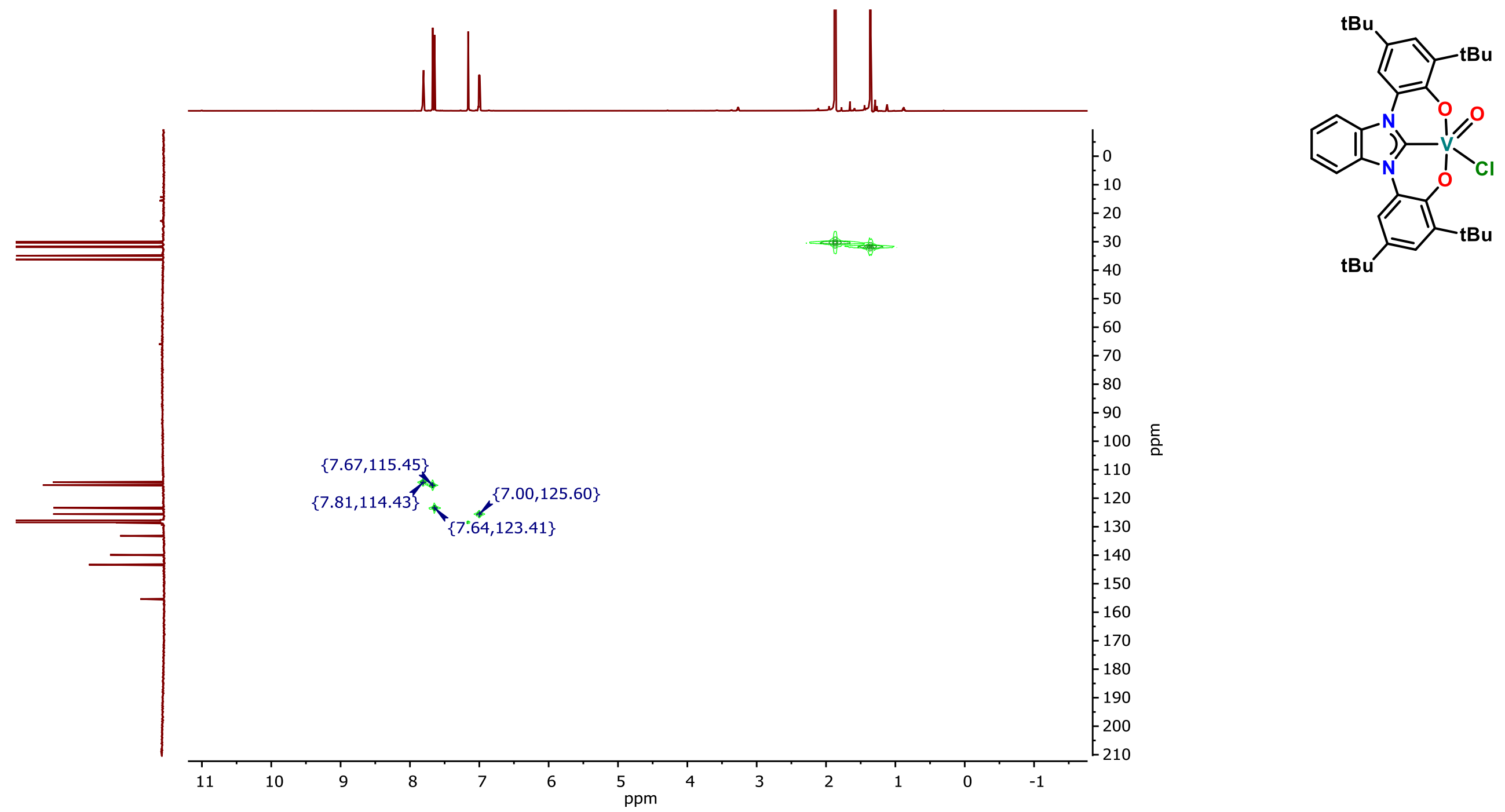

Figure S 9: $\quad{ }^{1} \mathrm{H}^{13}{ }^{13} \mathrm{CHSQC}$ of 2 in $\mathrm{C}_{6} \mathrm{D}_{6}$ at $298 \mathrm{~K}$. 

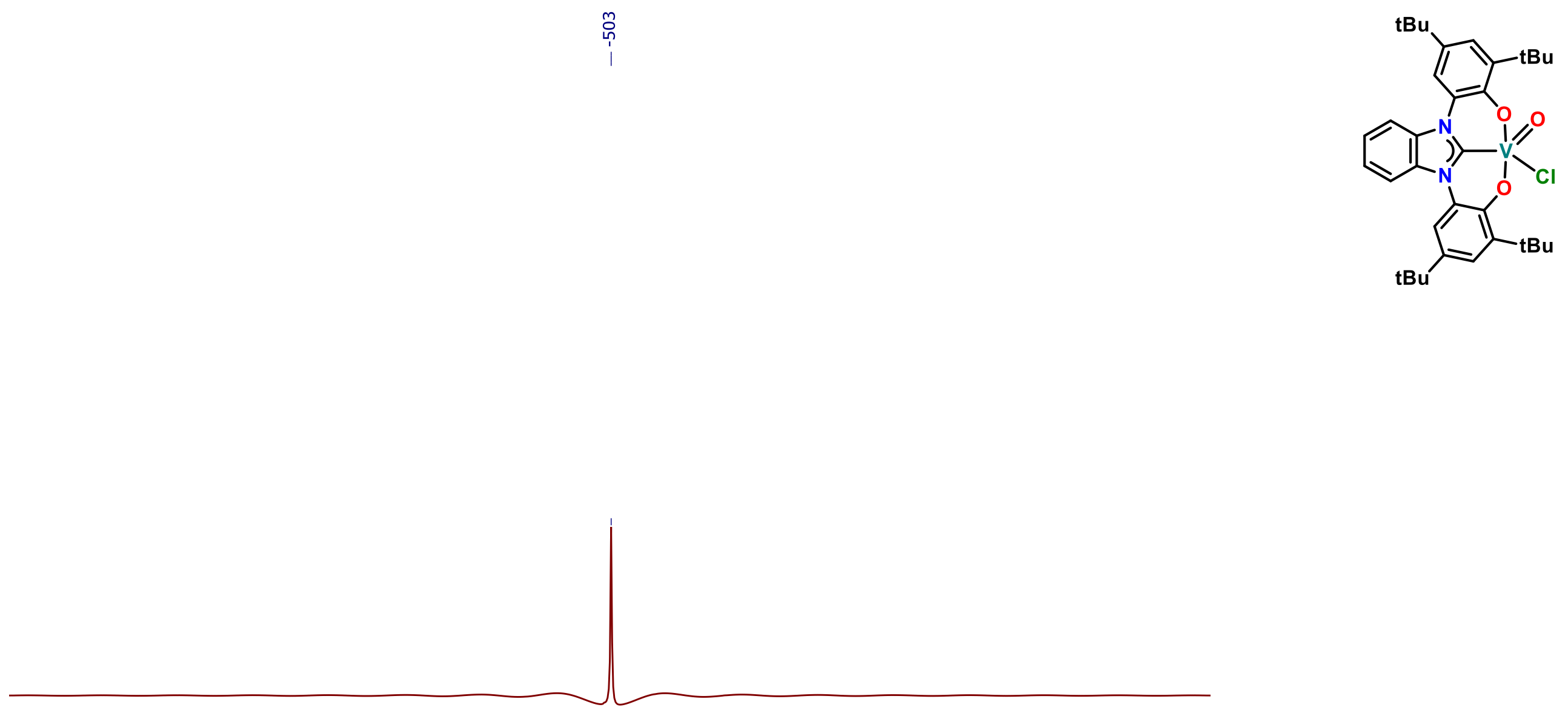

$\begin{array}{llllllllllllllllllllllllll}50 & 0 & -50 & -100 & -150 & -200 & -250 & -300 & -350 & -400 & -450 & -500 & -550 & -600 & -650 & -700 & -750 & -800 & -850 & -900 & -950 & -1000 & -1050\end{array}$

Figure S 10: $\quad{ }^{51} \mathrm{~V}$ NMR of 2 in $\mathrm{C}_{6} \mathrm{D}_{6}$ at $298 \mathrm{~K}$. 

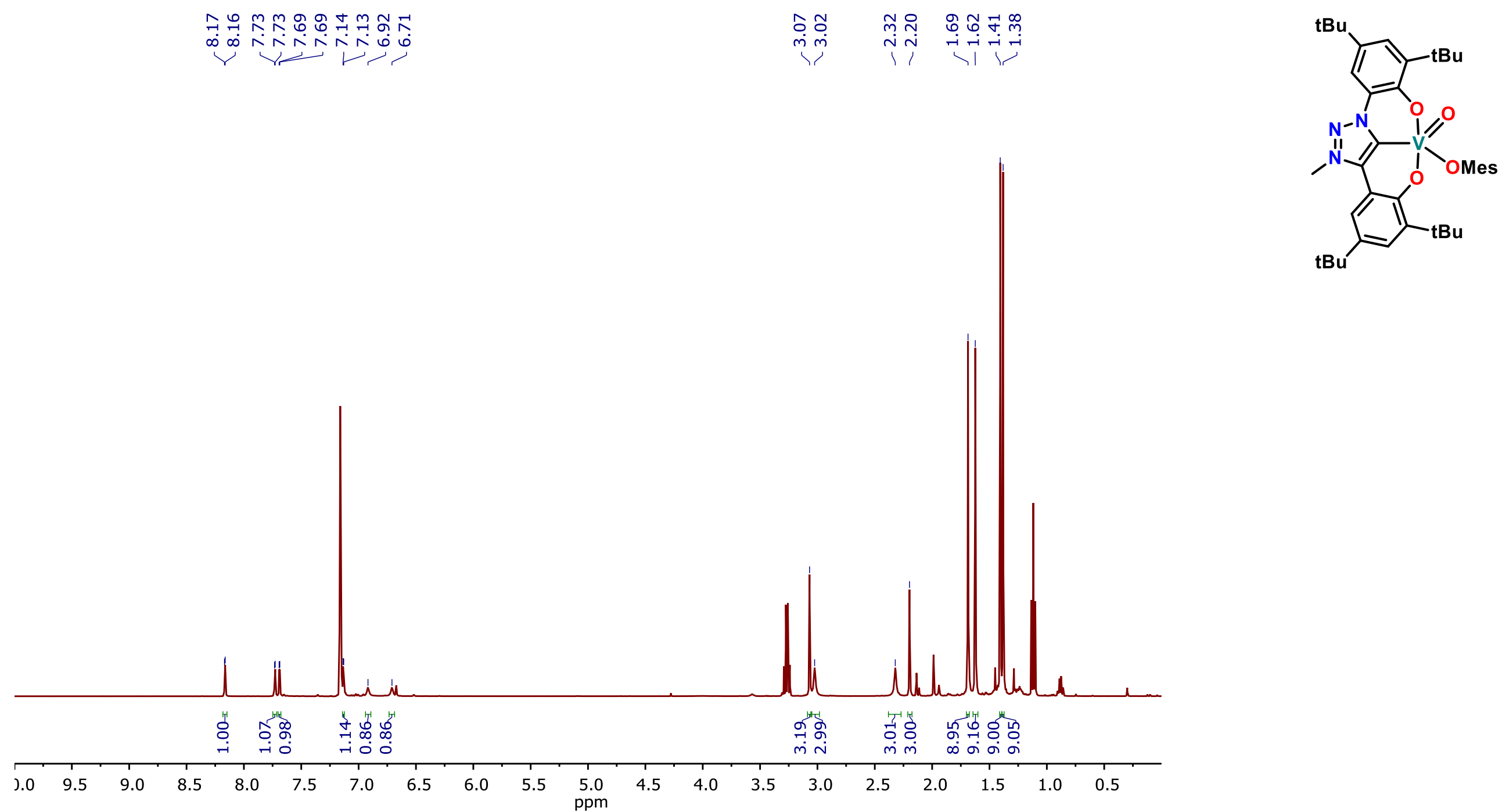

Figure $\mathrm{S}$ 11: $\quad{ }^{1} \mathrm{H}$ NMR of 3 in $\mathrm{C}_{6} \mathrm{D}_{6}$. Peaks at 6.672 .13 and $1.98 \mathrm{ppm}$ results from residual Mesitol. 

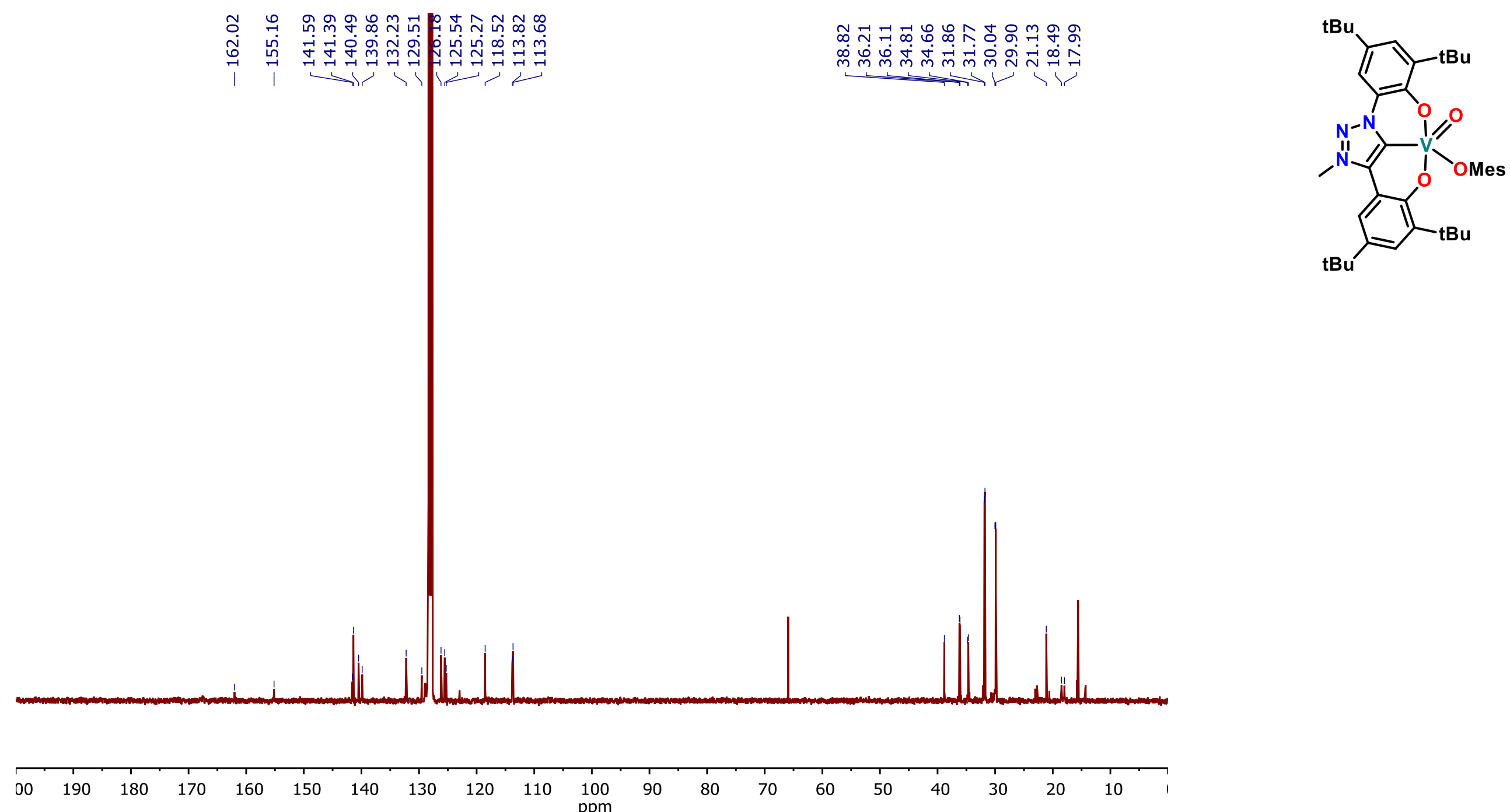

Figure $S$ 12: $\quad{ }^{13} \mathrm{C}$ NMR of $\mathbf{3}$ in $\mathrm{C}_{6} \mathrm{D}_{6}$ 

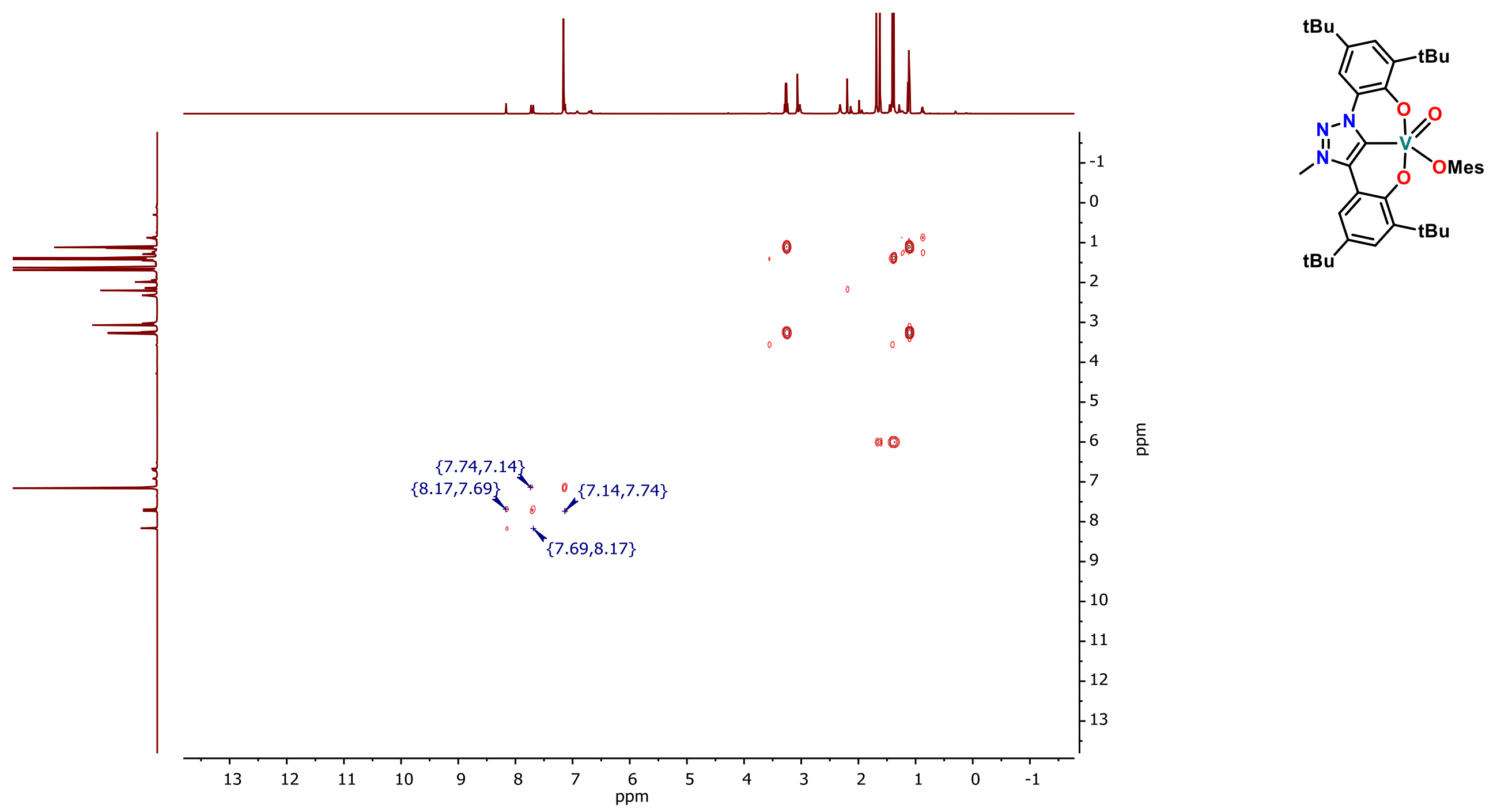

Figure $\mathrm{S}$ 13: $\quad{ }^{1} \mathrm{H}^{1} \mathrm{H}$ COSY of 3 in $\mathrm{C}_{6} \mathrm{D}_{6}$ 

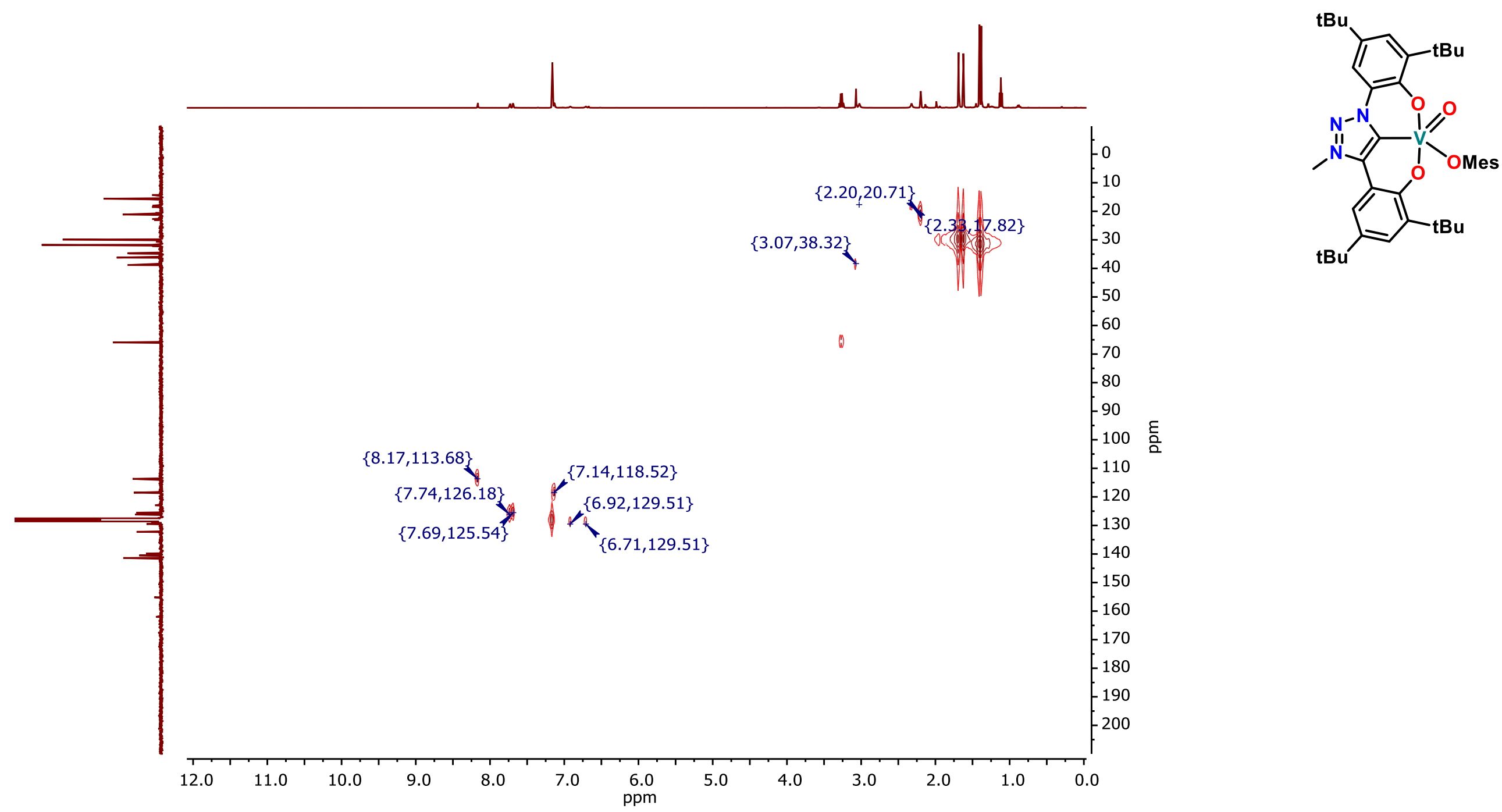

Figure $\mathrm{S} 14: \quad{ }^{1} \mathrm{H}^{13} \mathrm{C}$ HSQC of 3 in $\mathrm{C}_{6} \mathrm{D}_{6}$ 

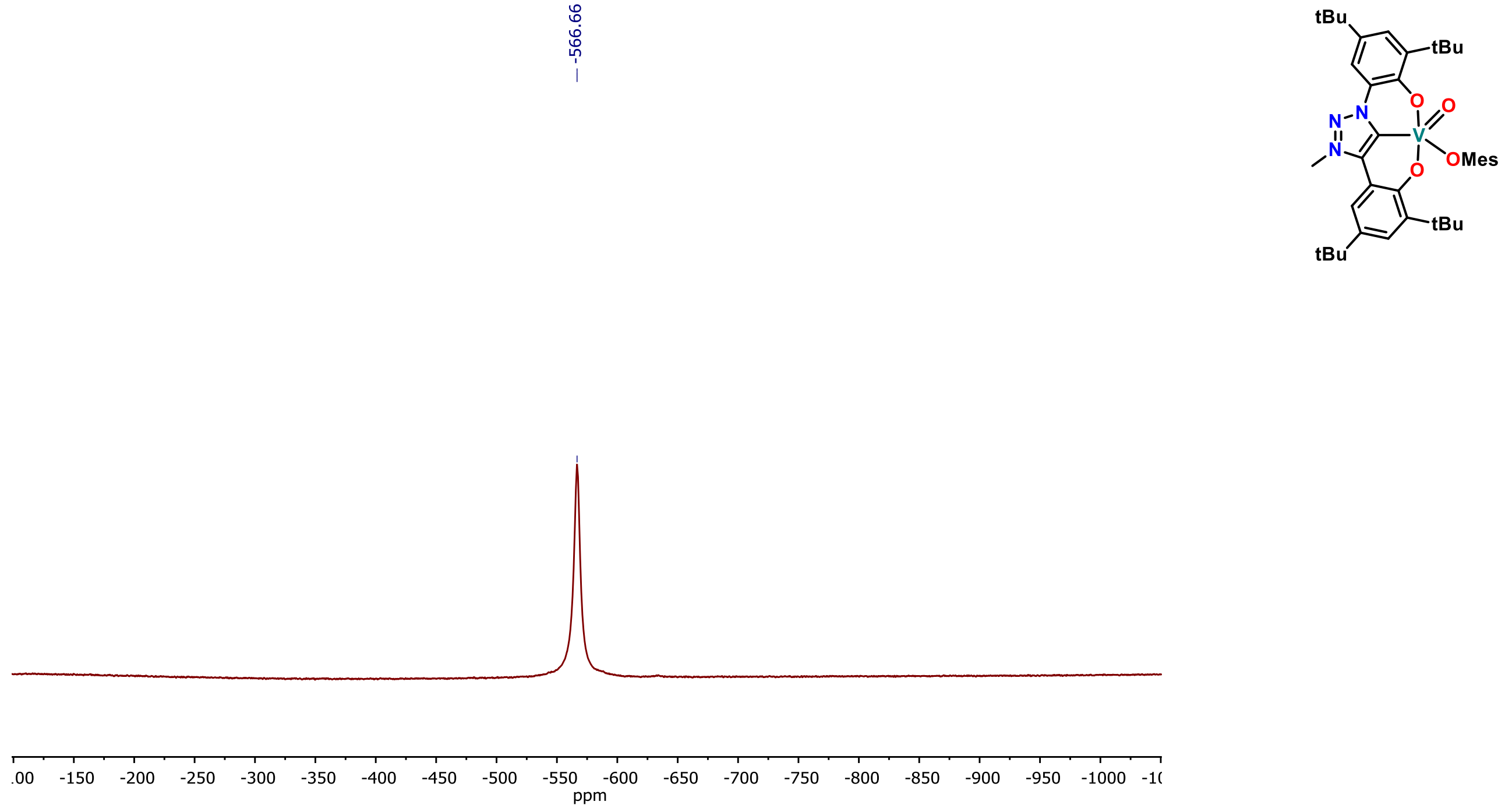

Figure S 15: $\quad{ }^{51} \mathrm{~V}$ NMR of $\mathbf{3}$ in $\mathrm{C}_{6} \mathrm{D}_{6}$ 


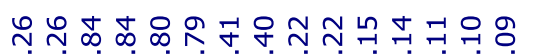

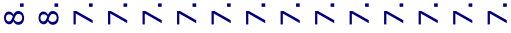

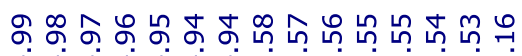

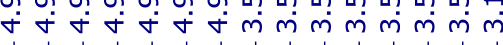

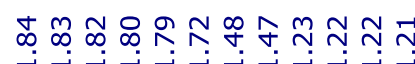

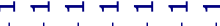

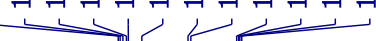

tBu

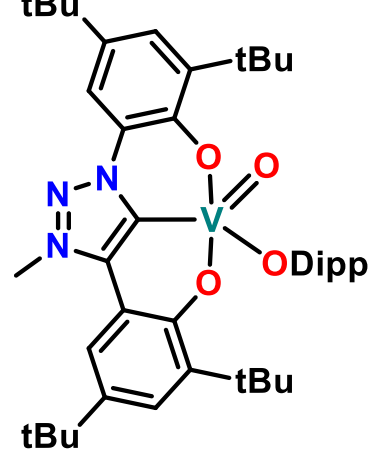

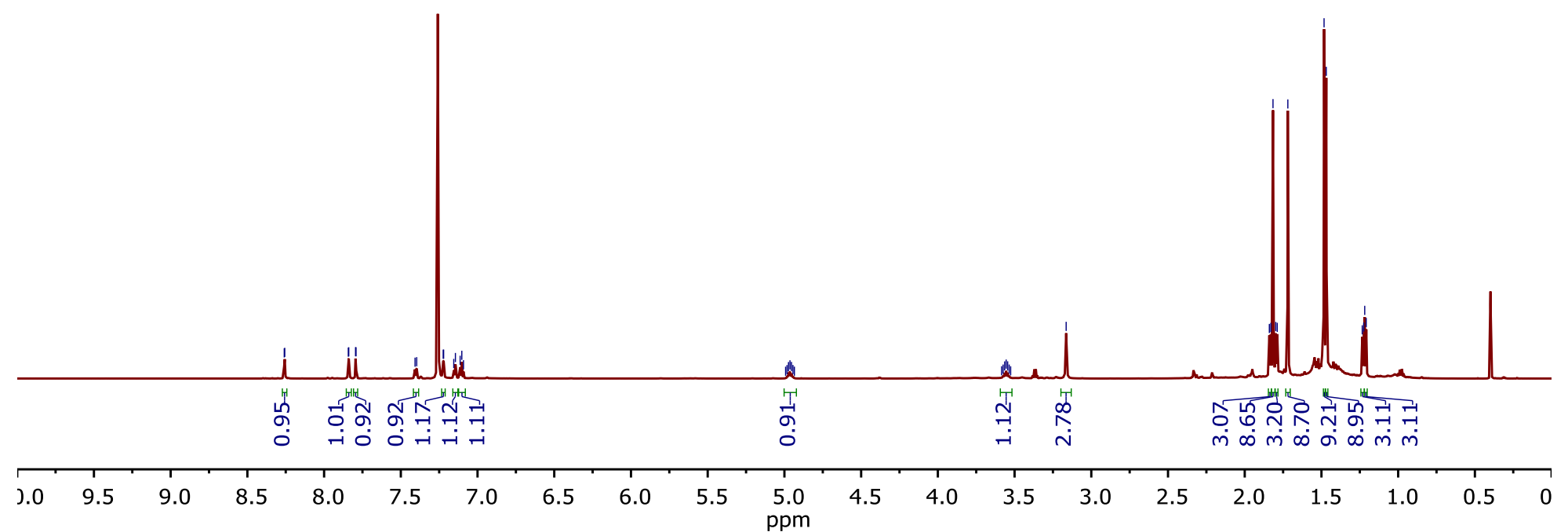

Figure $S 16:{ }^{1} \mathrm{H}$ NMR of 4 in $\mathrm{C}_{6} \mathrm{D}_{6}$ at $298 \mathrm{~K}$. 

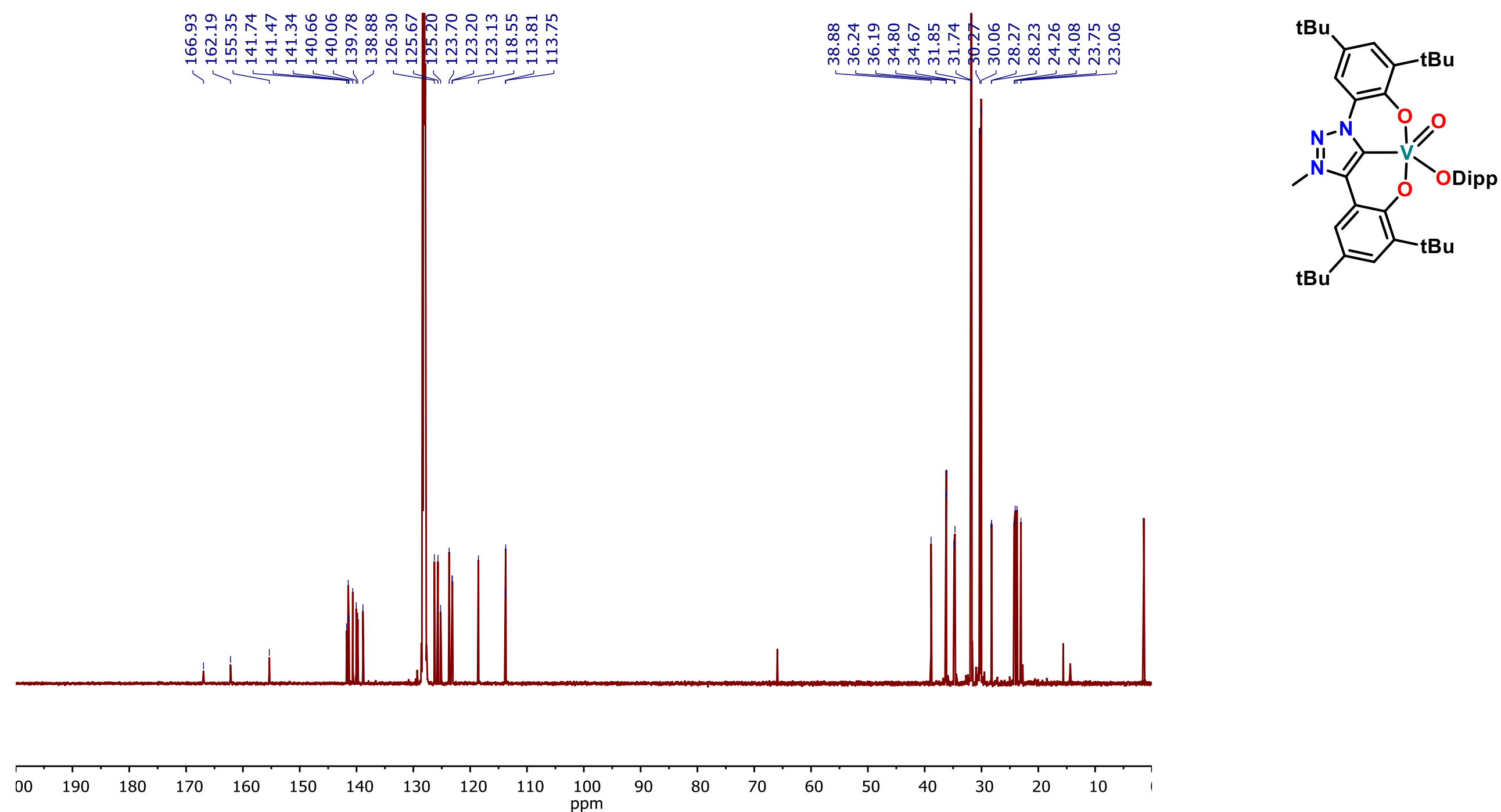

Figure $S$ 17: $\quad{ }^{13} \mathrm{C}$ NMR of 4 in $C_{6} D_{6}$ at $298 \mathrm{~K}$. 

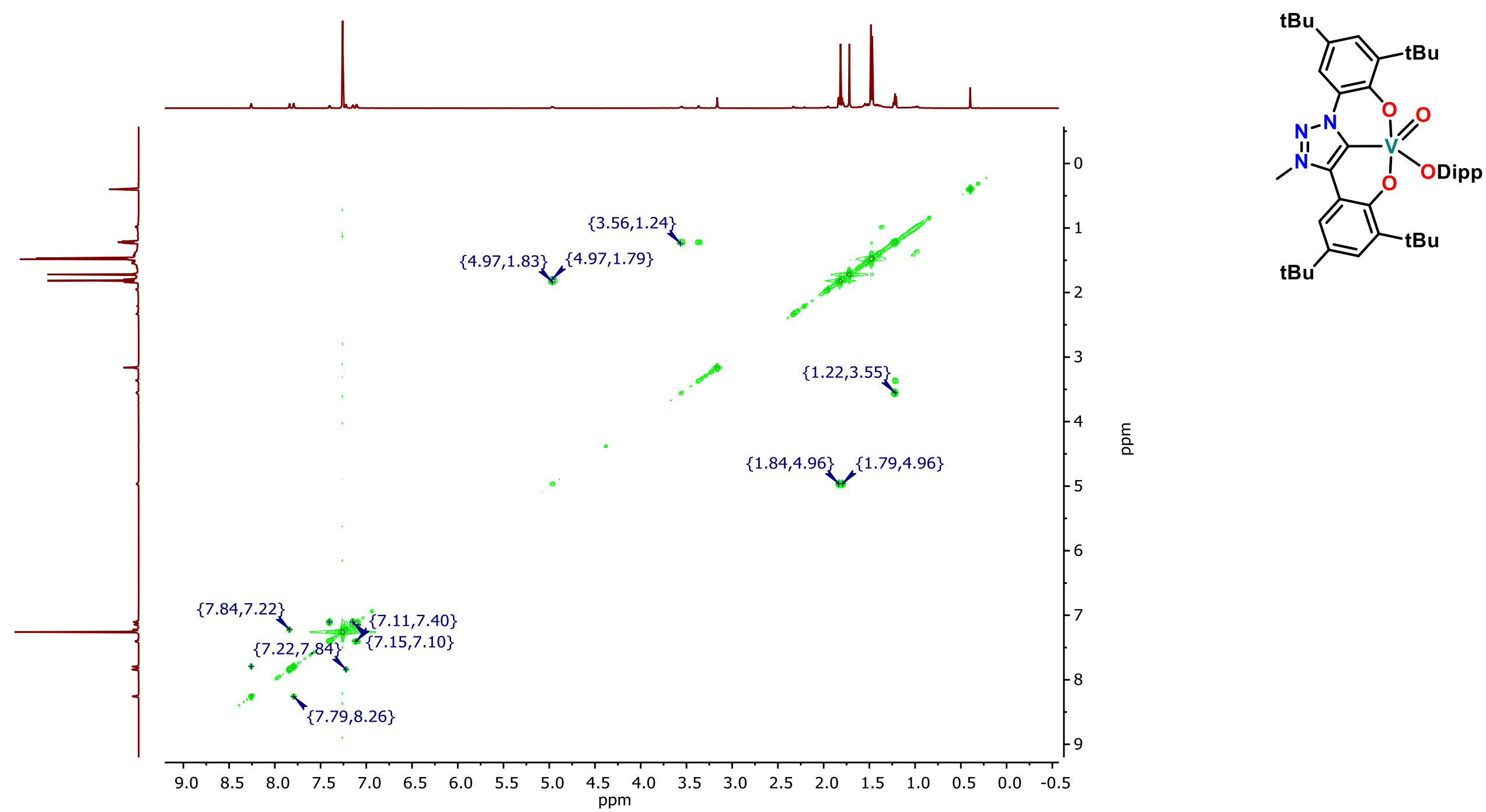

Figure $\mathrm{S} 18: \quad{ }^{1} \mathrm{H}-{ }^{1} \mathrm{H}$ COSY of 4 in $\mathrm{C}_{6} \mathrm{D}_{6}$ at $298 \mathrm{~K}$ 

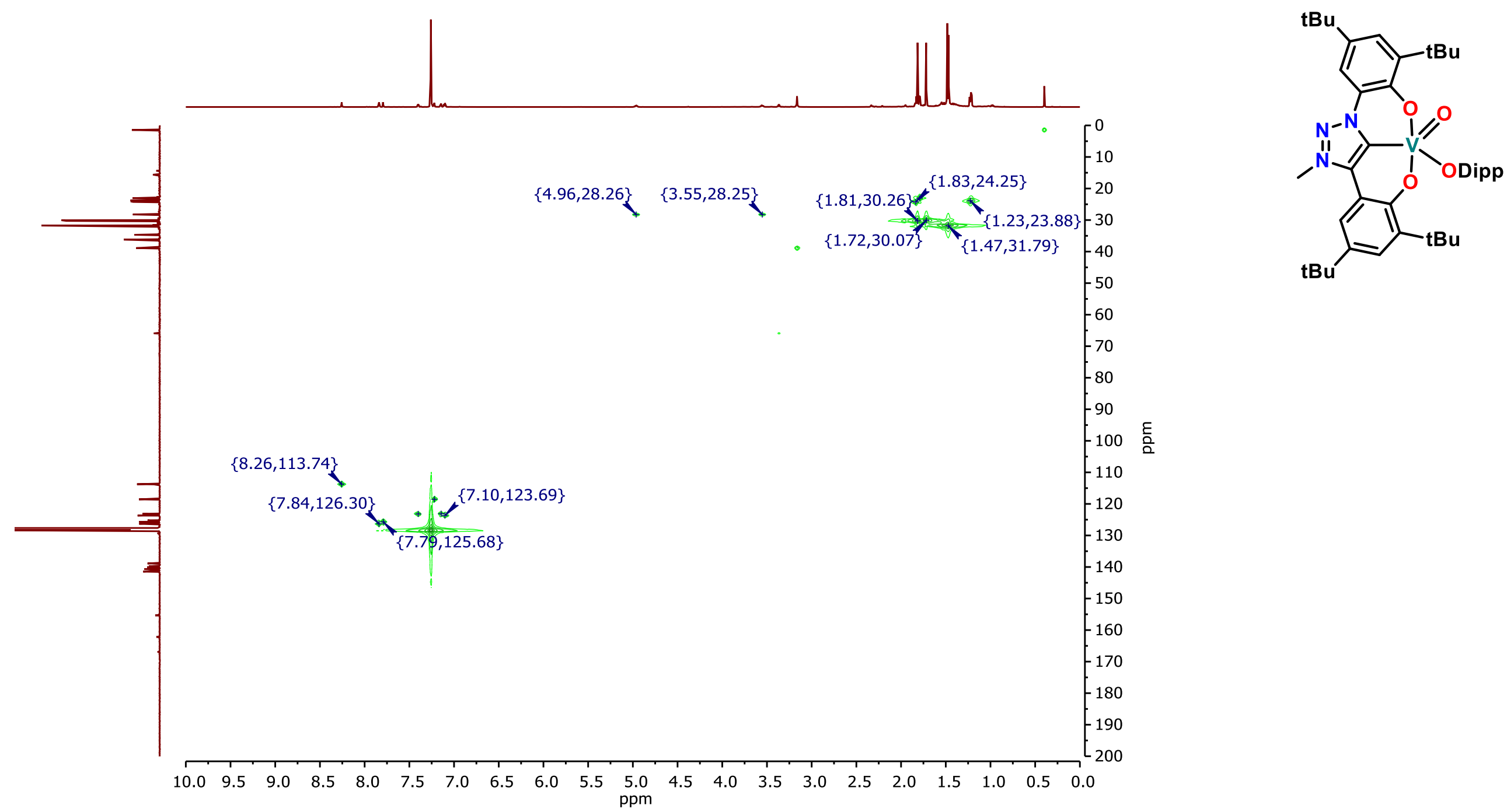

Figure $\mathrm{S}$ 19: $\quad{ }^{1} \mathrm{H}^{-13} \mathrm{C}$ HSQC of 4 in $\mathrm{C}_{6} \mathrm{D}_{6}$ at $298 \mathrm{~K}$ 

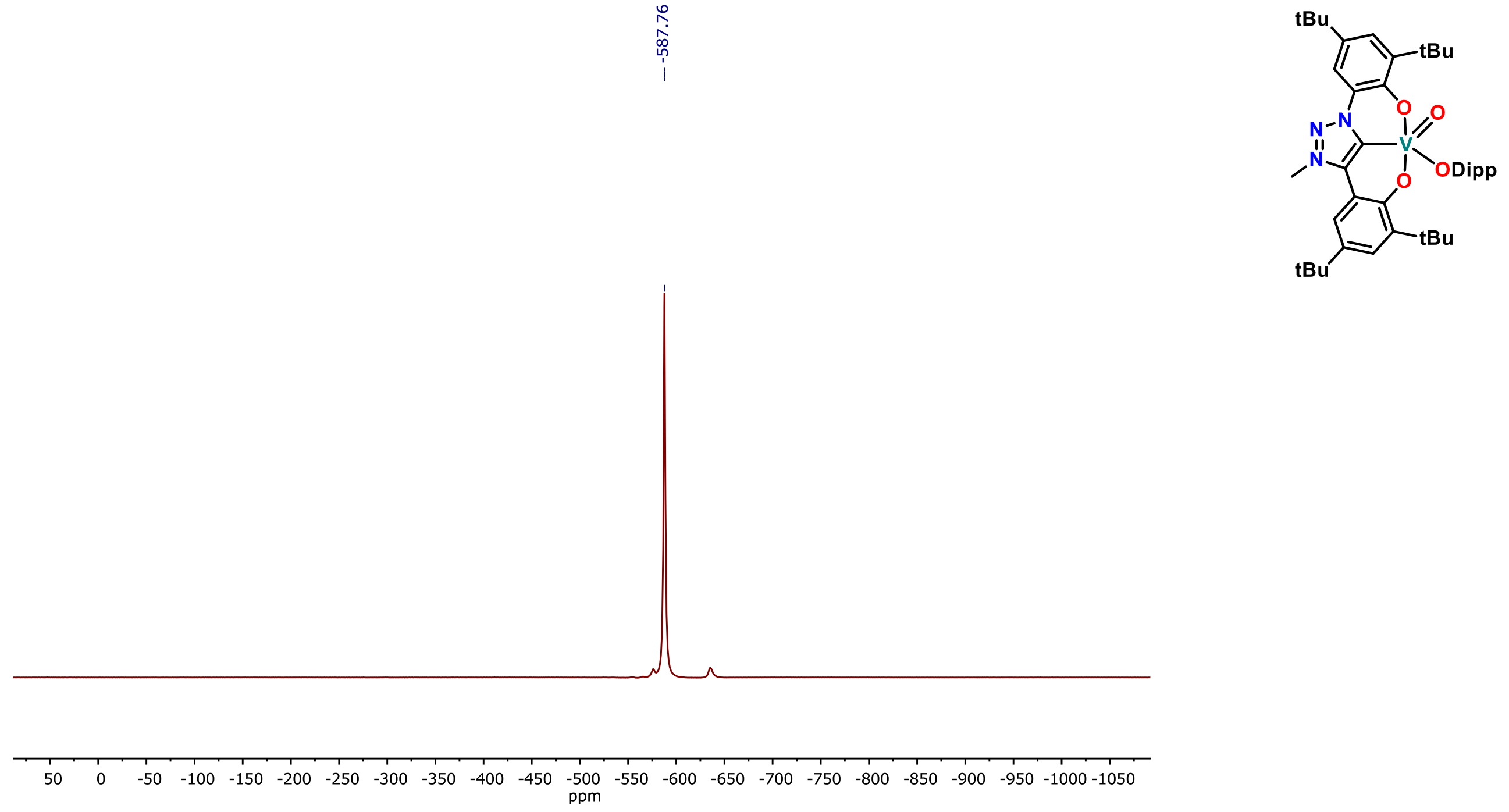

Figure S 20: $\quad{ }^{51} \mathrm{~V}$ NMR of 4 in $\mathrm{C}_{6} \mathrm{D}_{6}$ at $298 \mathrm{~K}$ 


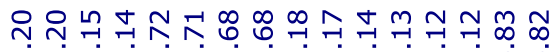

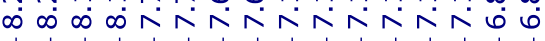

年

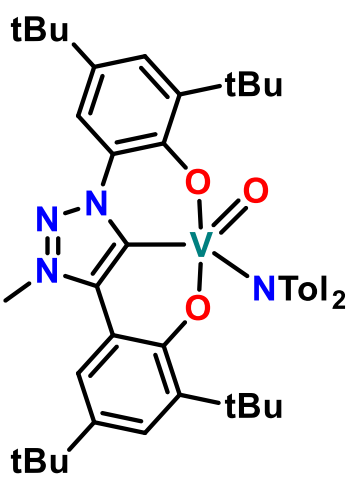

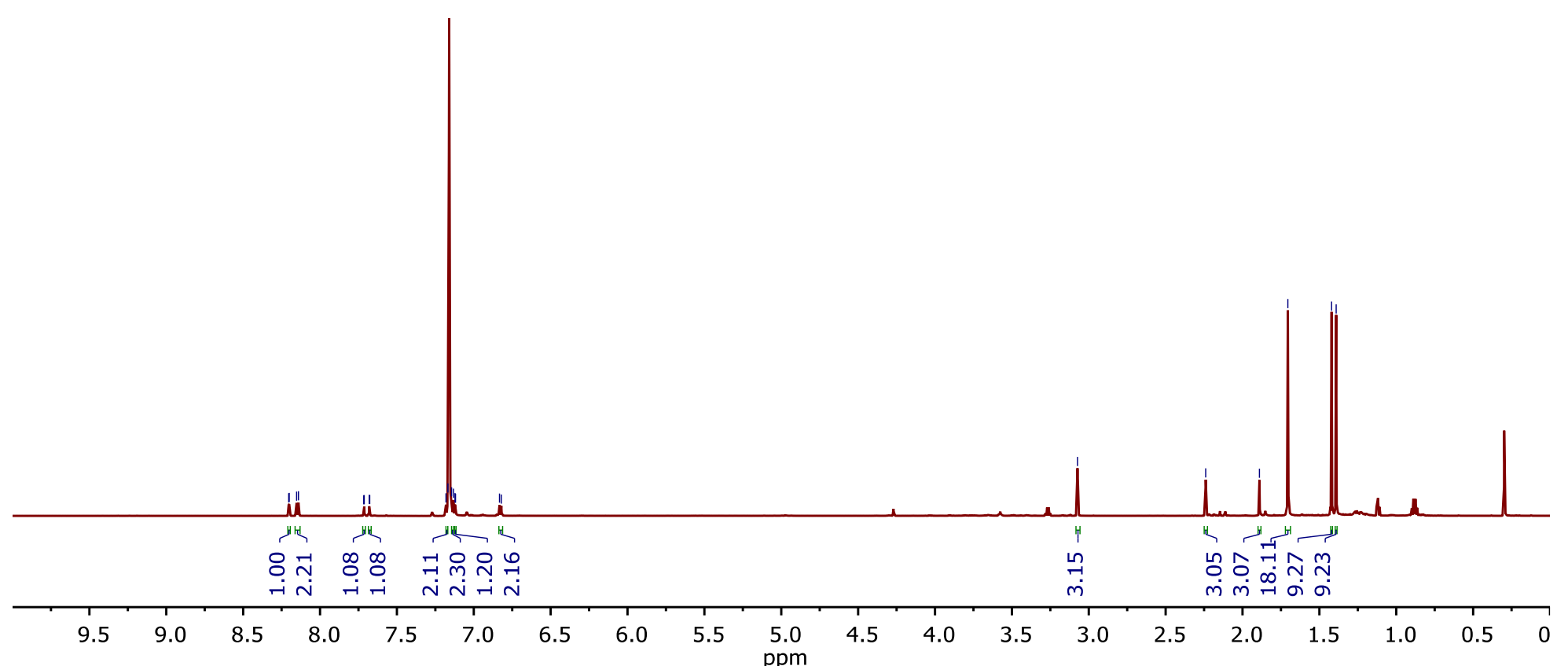

Figure $\mathrm{S} 21: \quad{ }^{1} \mathrm{H}-\mathrm{NMR}$ of 5 in $\mathrm{C}_{6} \mathrm{D}_{6}$ at $298 \mathrm{~K}$ 

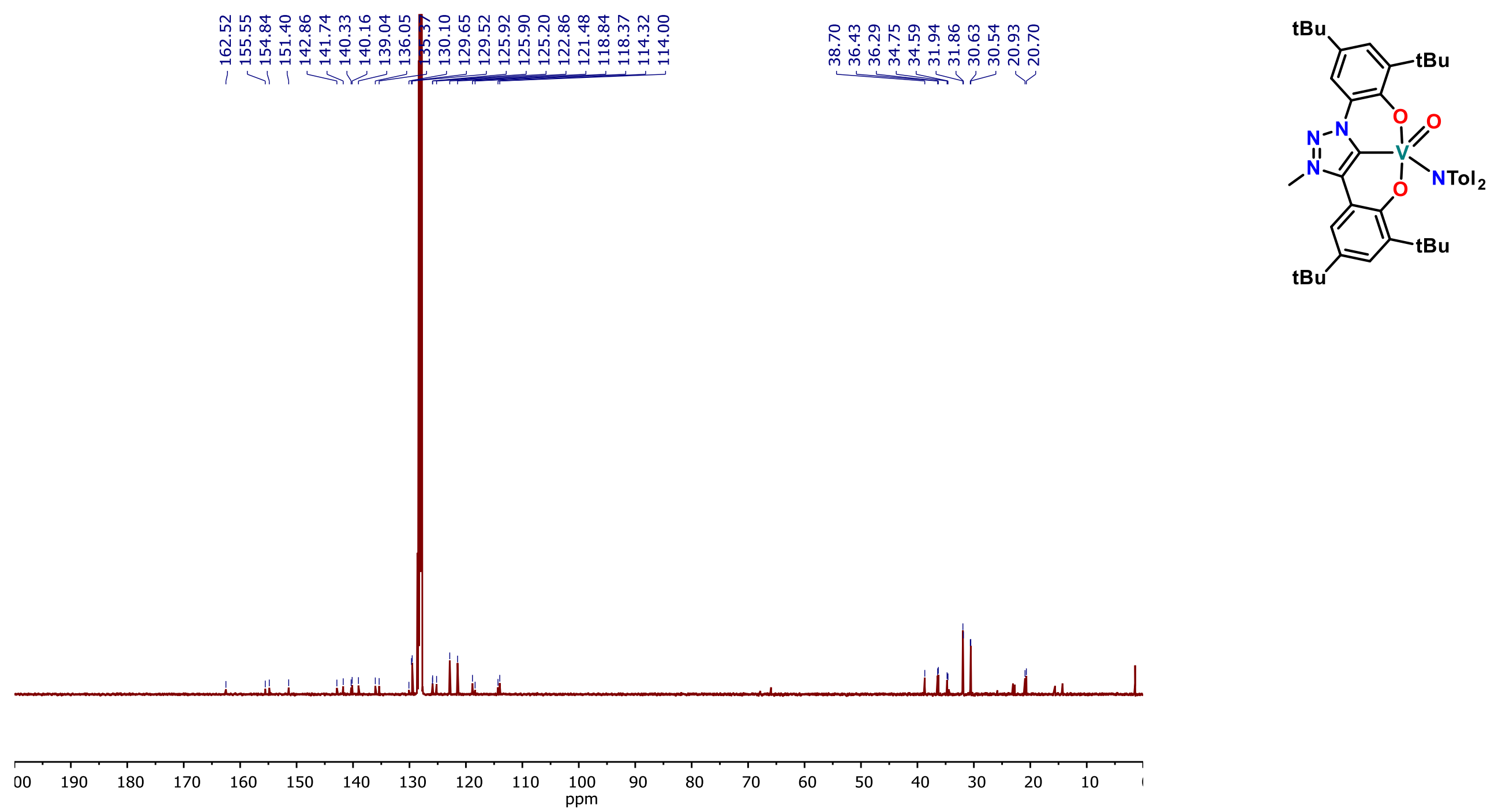

Figure $S$ 22: $\quad{ }^{13} \mathrm{C}$ NMR of 5 in $\mathrm{C}_{6} \mathrm{D}_{6}$ at $298 \mathrm{~K}$ 

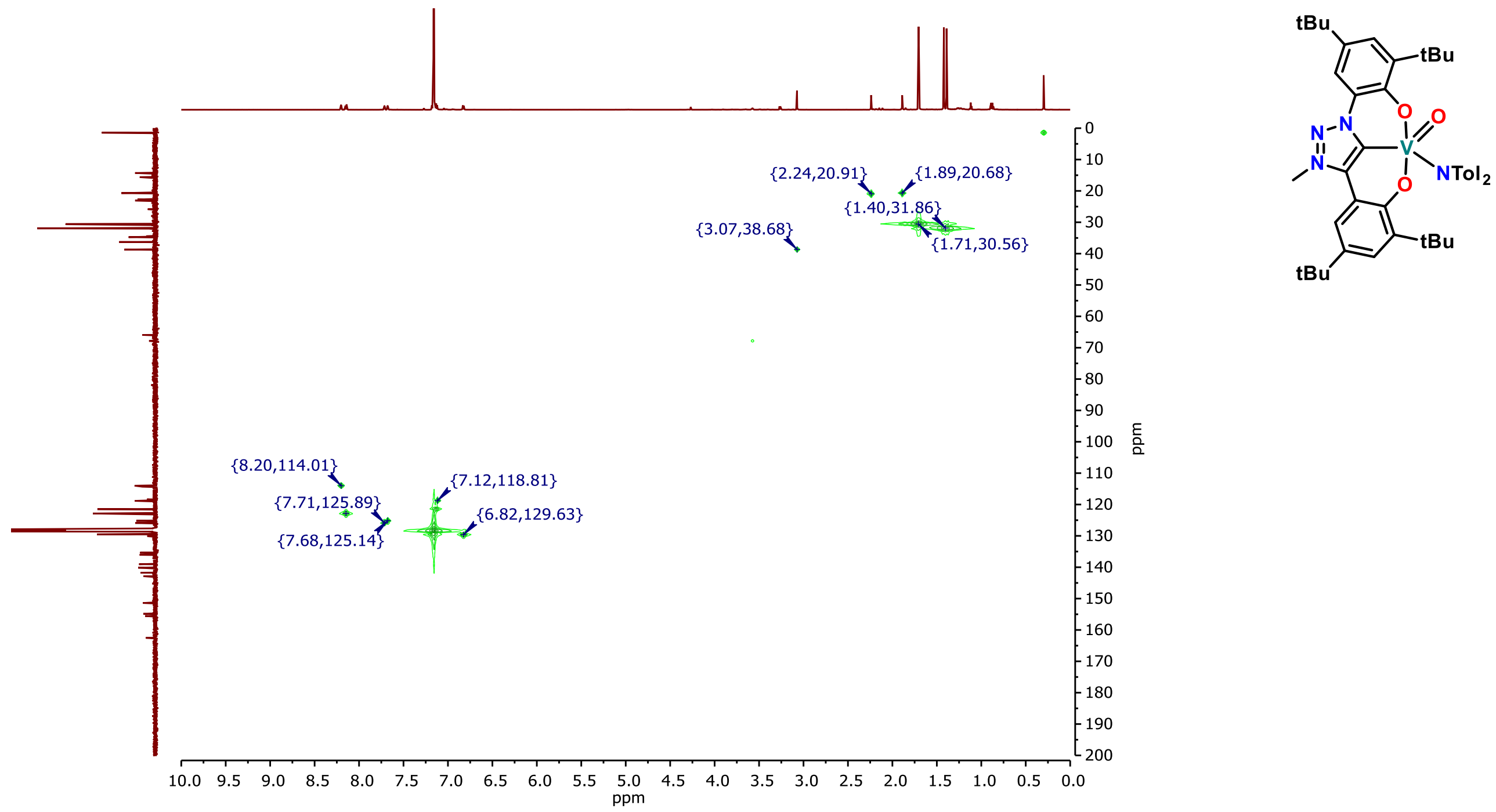

Figure $\mathrm{S} 23: \quad{ }^{1} \mathrm{H}-{ }^{1} \mathrm{H}$ COSY of 5 in $\mathrm{C}_{6} \mathrm{D}_{6}$ at $298 \mathrm{~K}$ 

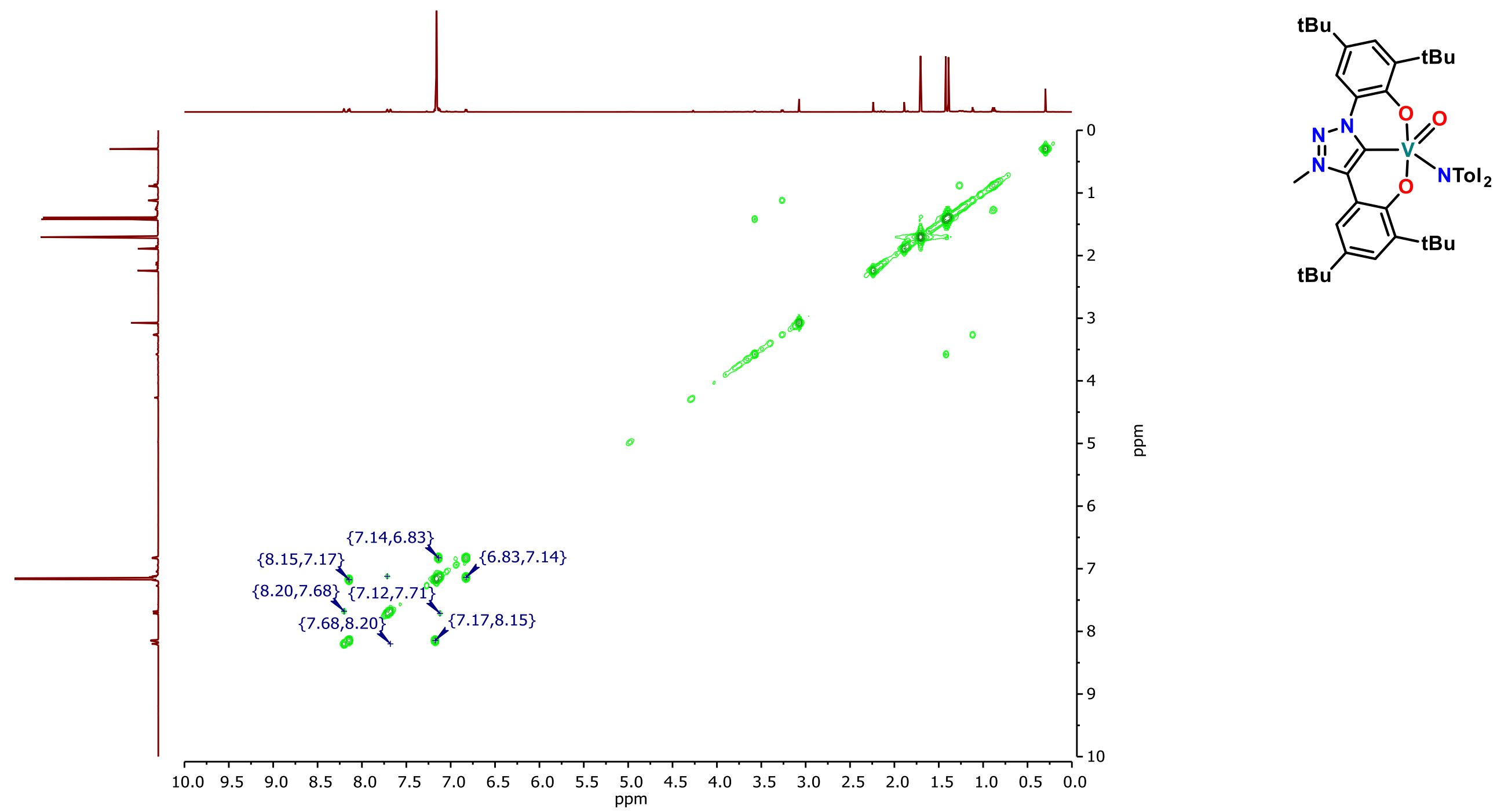

Figure $\mathrm{S} 24: \quad{ }^{1} \mathrm{H}^{13}{ }^{13} \mathrm{HSOC}$ of 5 in $\mathrm{C}_{6} \mathrm{D}_{6}$ at $298 \mathrm{~K}$ 

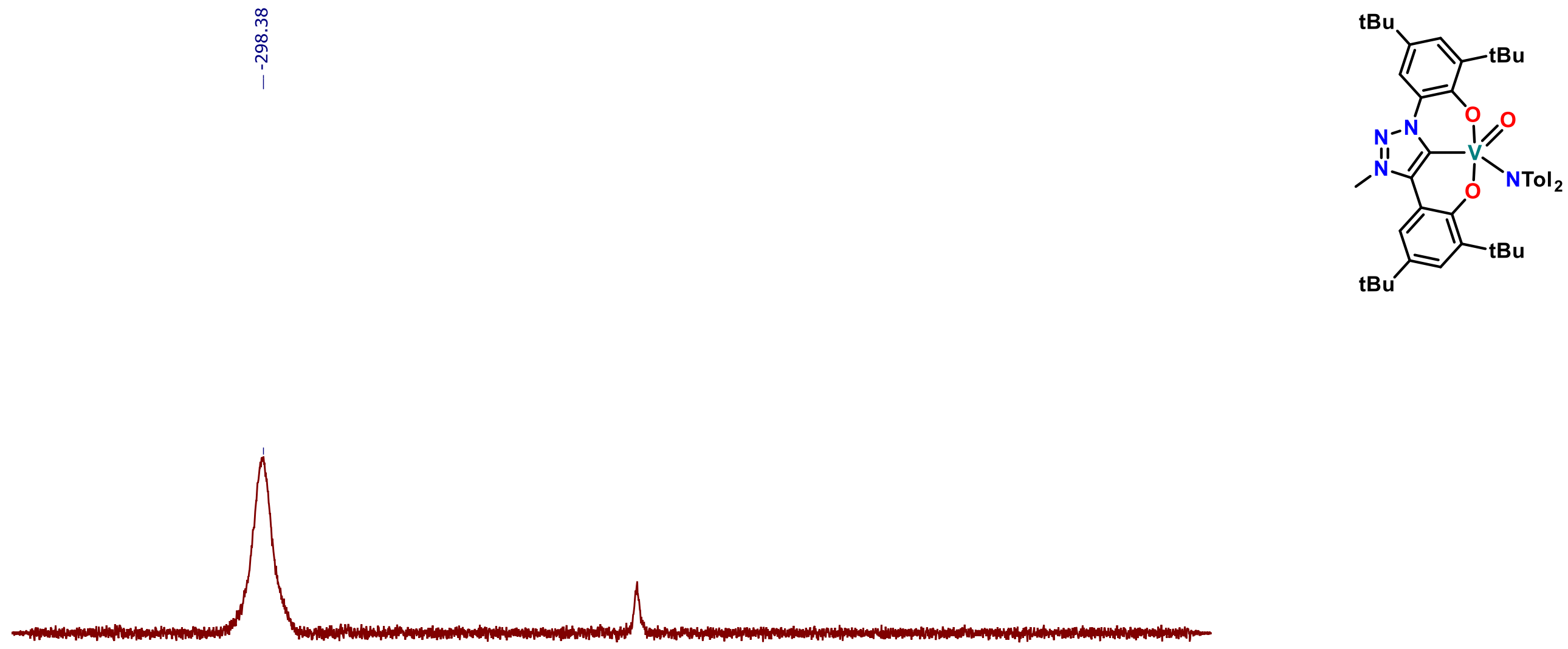

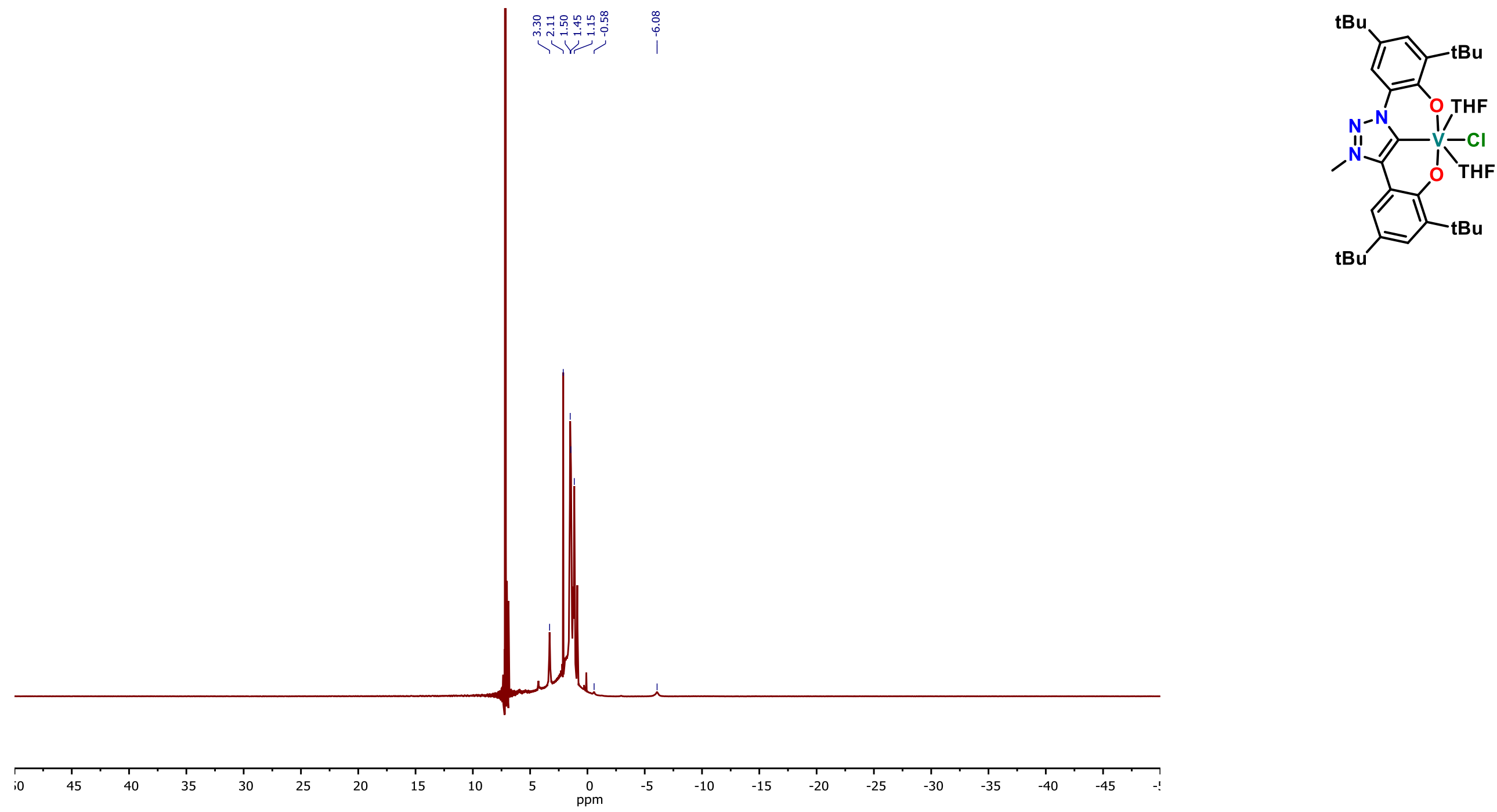

Figure S 26: $\quad{ }^{1} \mathrm{H}$ NMR of 6 in $\mathrm{C}_{6} \mathrm{D}_{6}$ at $298 \mathrm{~K}$. 

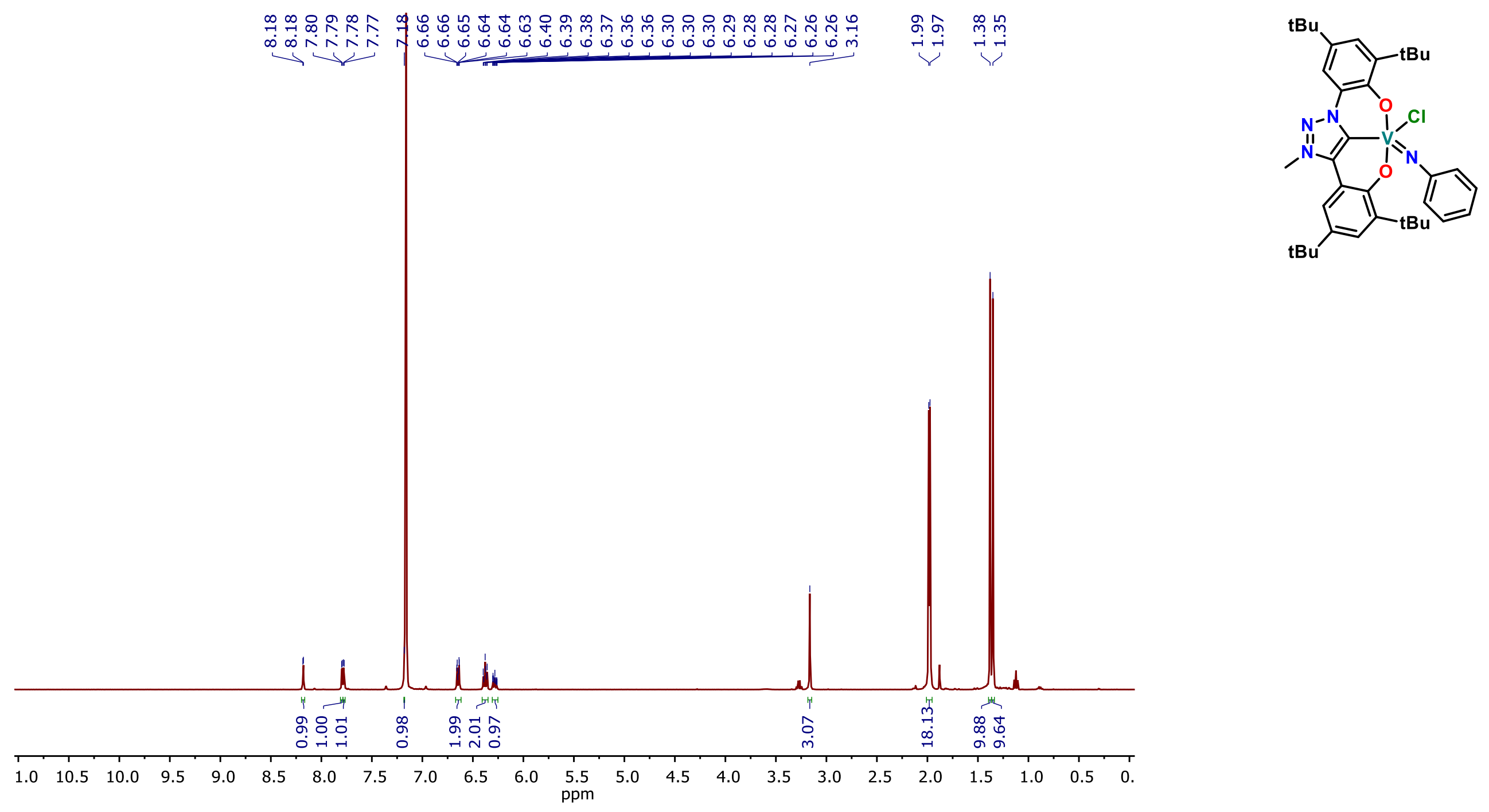

Figure $S 27: \quad{ }^{1} \mathrm{H}$ NMR of 7 in $\mathrm{C}_{6} \mathrm{D}_{6}$ at $298 \mathrm{~K}$. 


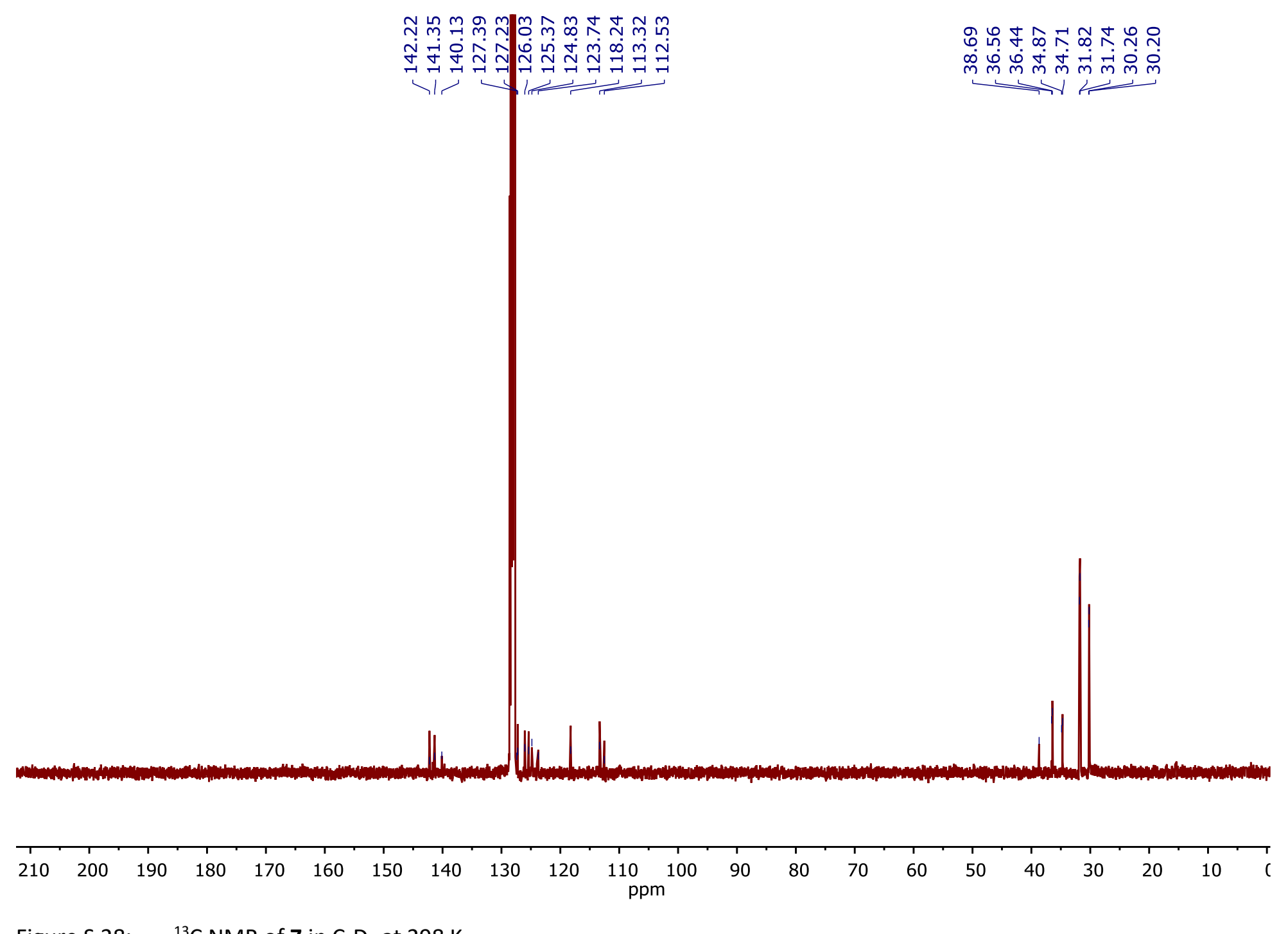



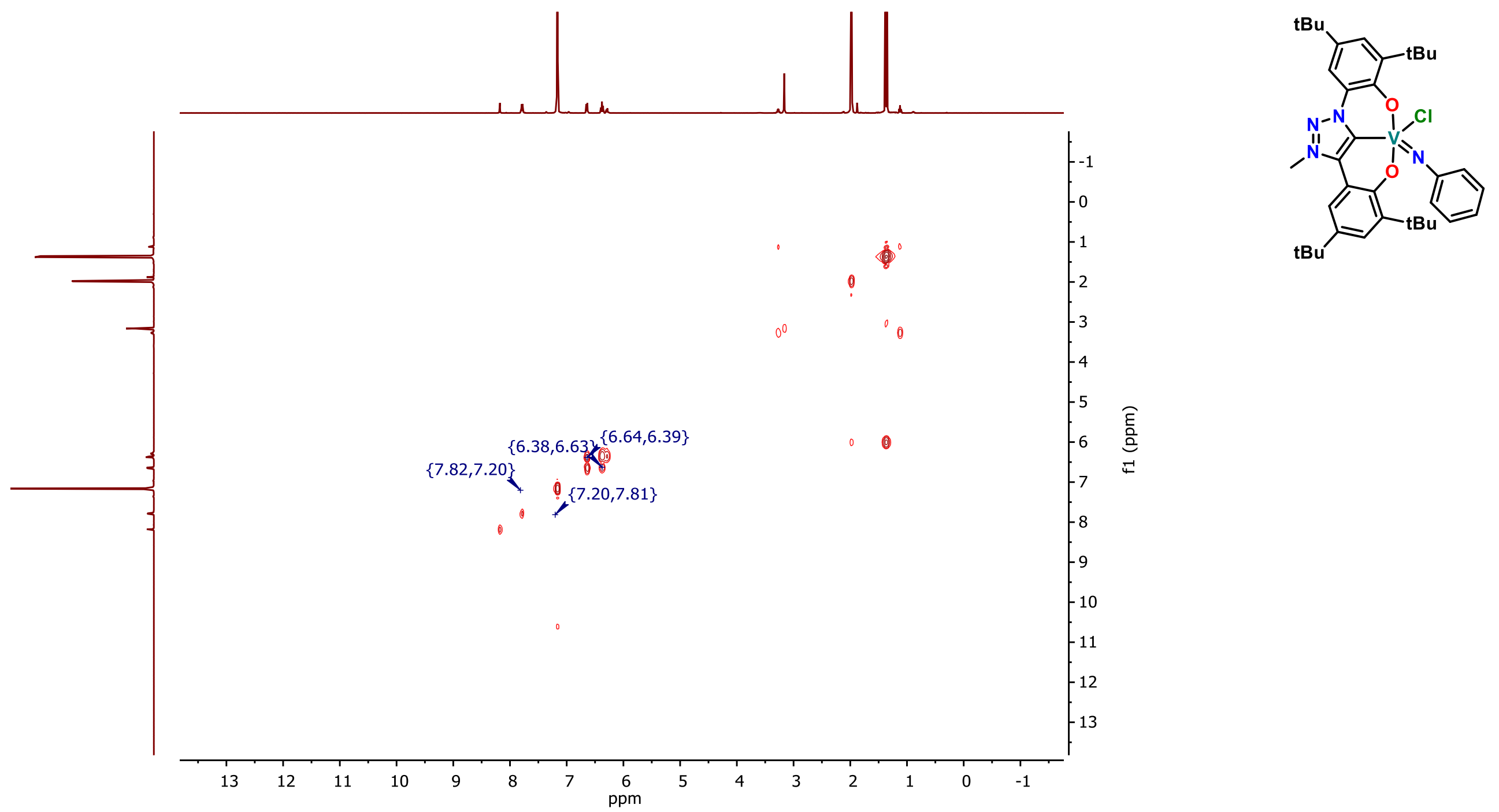

Figure $S$ 29: $\quad{ }^{1} \mathrm{H}^{1} \mathrm{H}$ COSY NMR of 7 in $\mathrm{C}_{6} \mathrm{D}_{6}$ at $298 \mathrm{~K}$. 

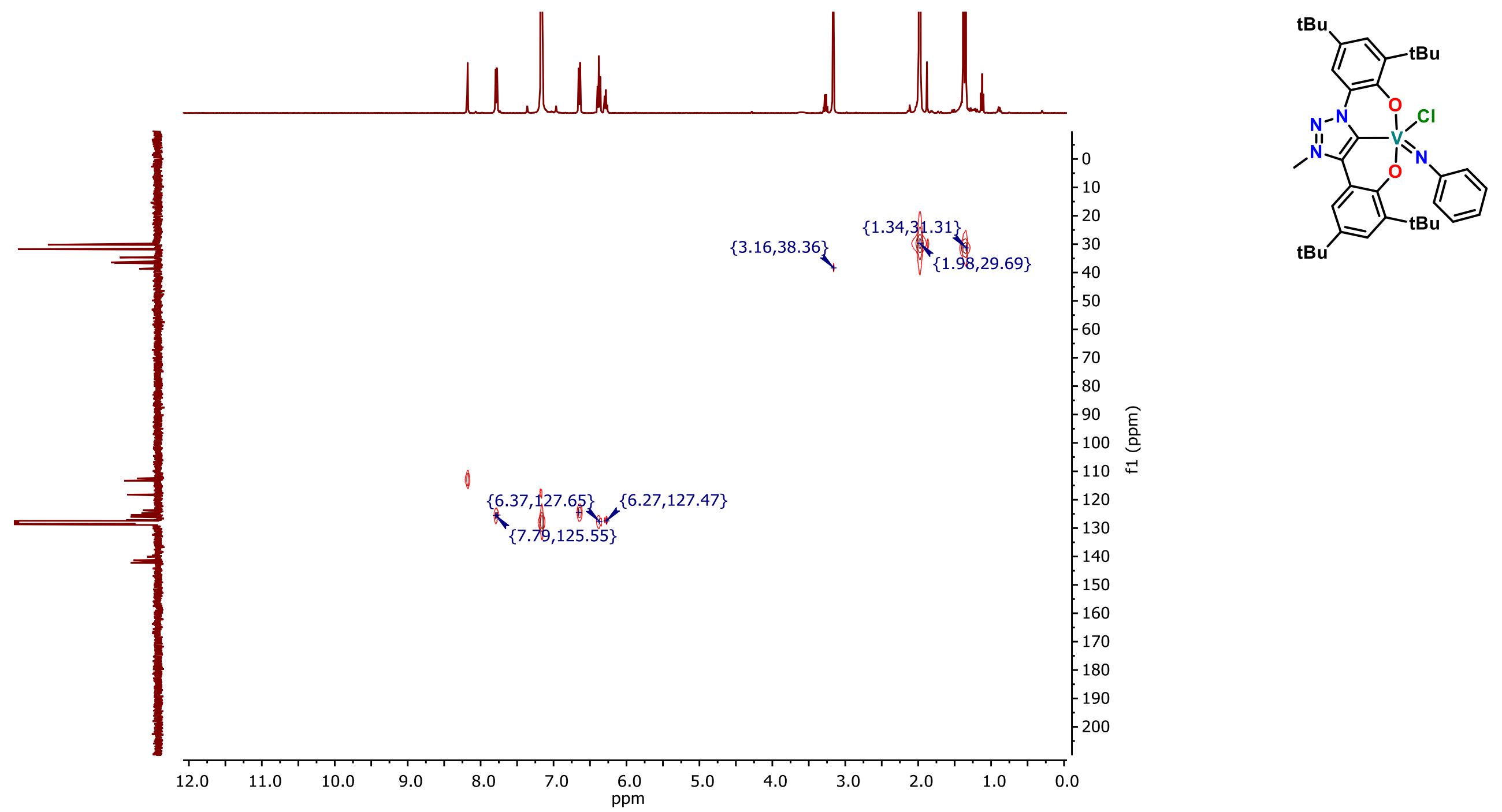

Figure $\mathrm{S} 30: \quad{ }^{1} \mathrm{H}^{13} \mathrm{C}$ HSQC NMR of 7 in $\mathrm{C}_{6} \mathrm{D}_{6}$ at $298 \mathrm{~K}$. 

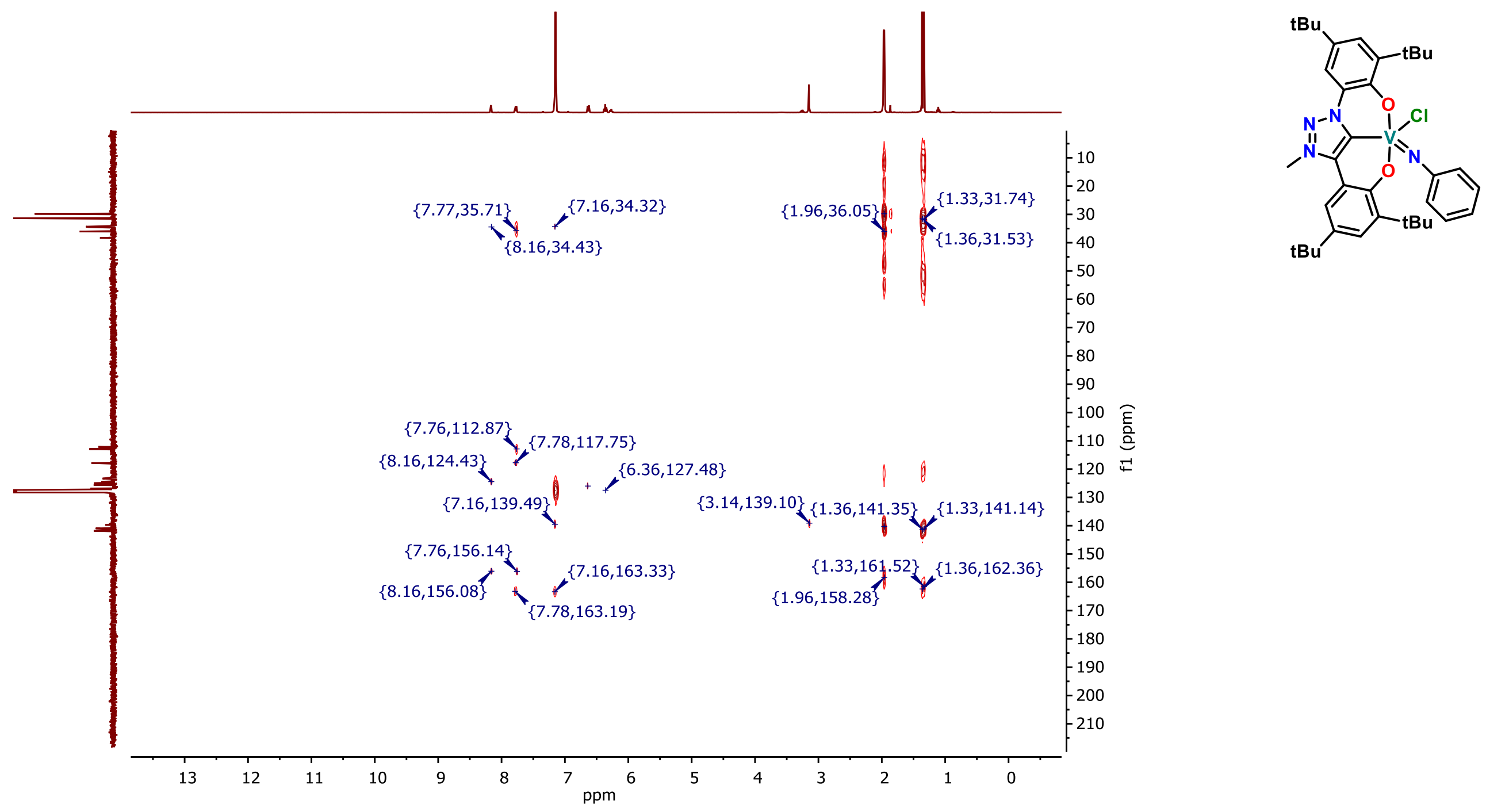

Figure $\mathrm{S} 31: \quad{ }^{1} \mathrm{H}^{13} \mathrm{C}$ HMBC NMR of 7 in $\mathrm{C}_{6} \mathrm{D}_{6}$ at $298 \mathrm{~K}$. 

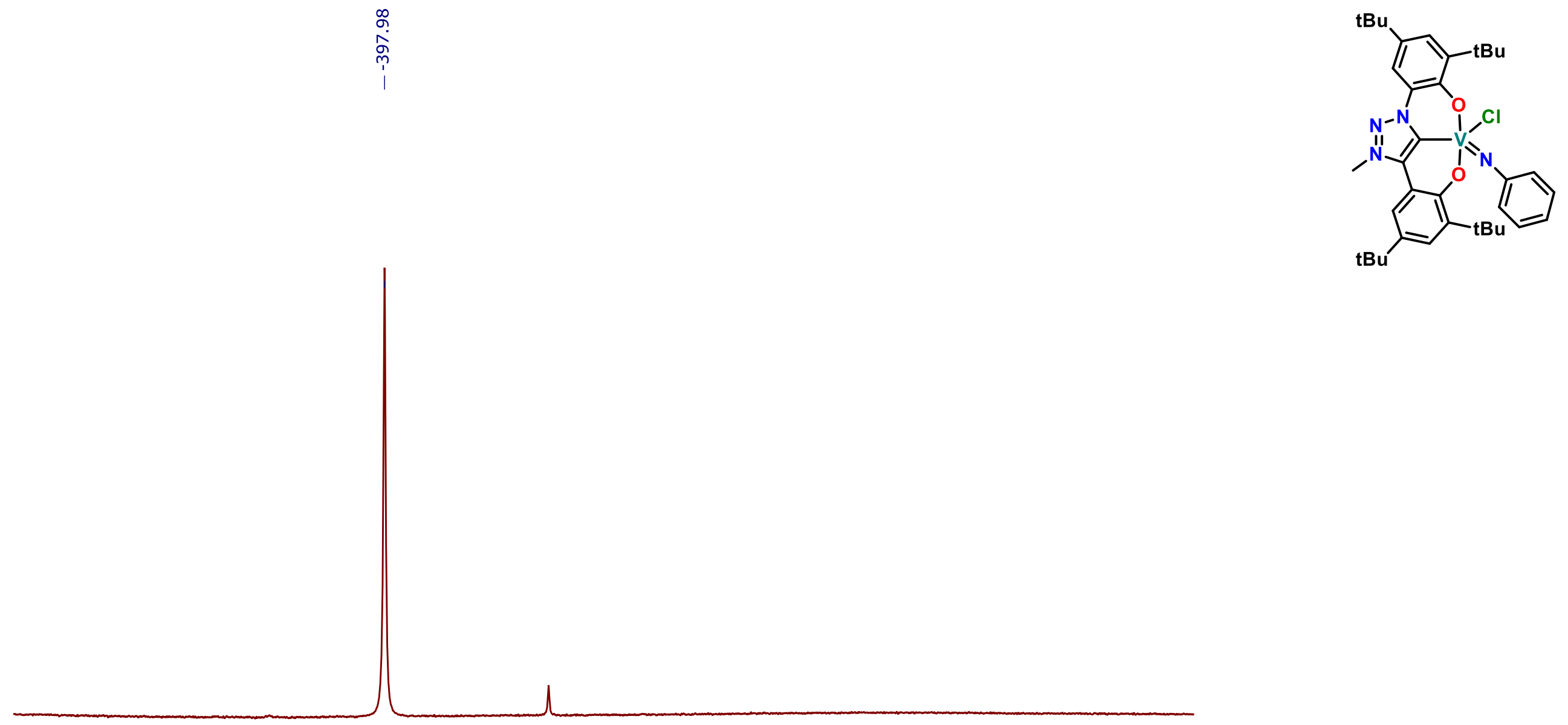

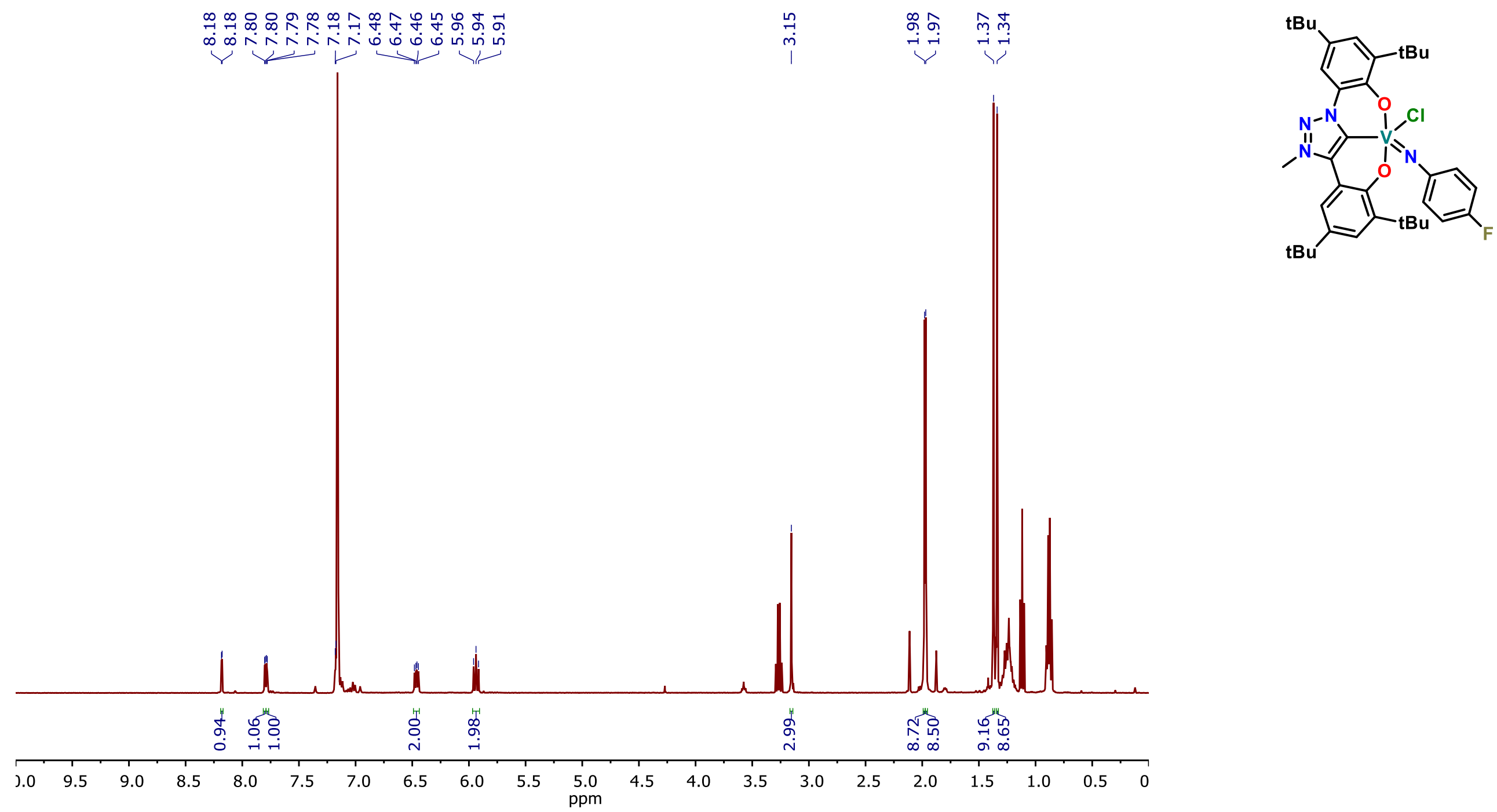

Figure $S$ 33: $\quad{ }^{1} \mathrm{H}$ NMR of 8 in $\mathrm{C}_{6} \mathrm{D}_{6}$ 


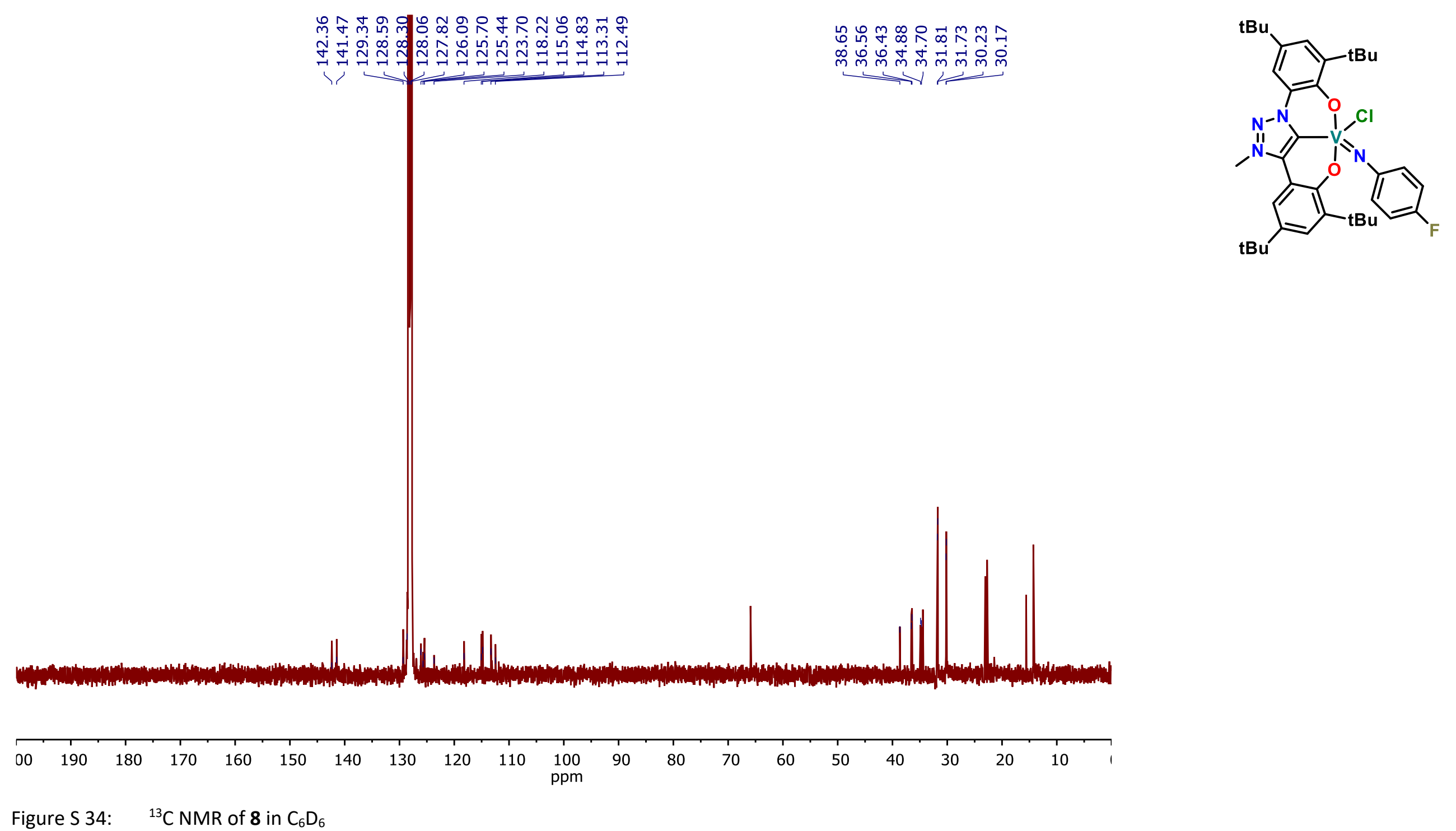



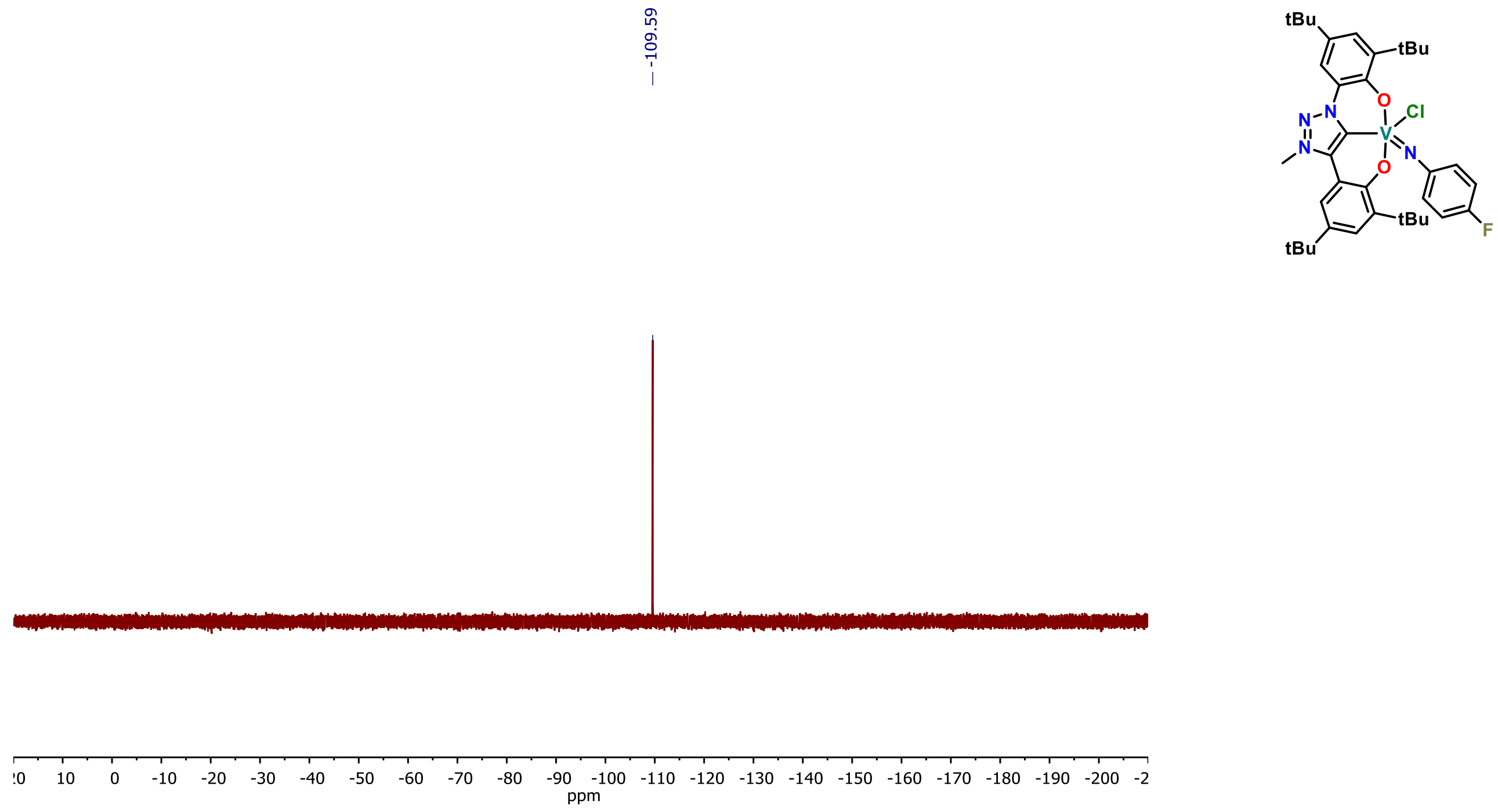

Figure $S 35: \quad{ }^{19} \mathrm{~F}$ NMR of 8 in $\mathrm{C}_{6} \mathrm{D}_{6}$ 

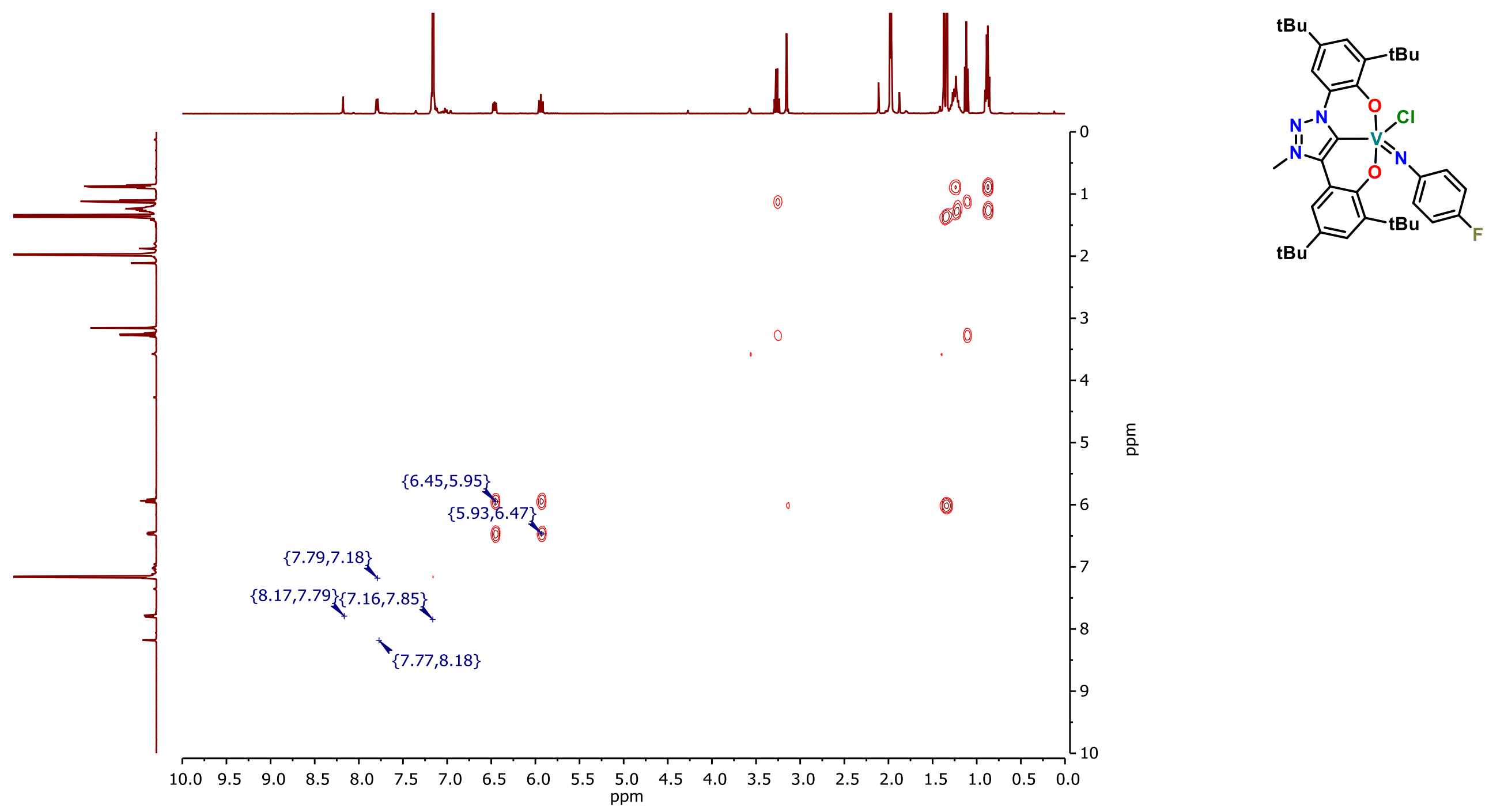

Figure $S$ 36: $\quad{ }^{1} \mathrm{H}^{1} \mathrm{H}$ COSY of 8 in $\mathrm{C}_{6} \mathrm{D}_{6}$ 

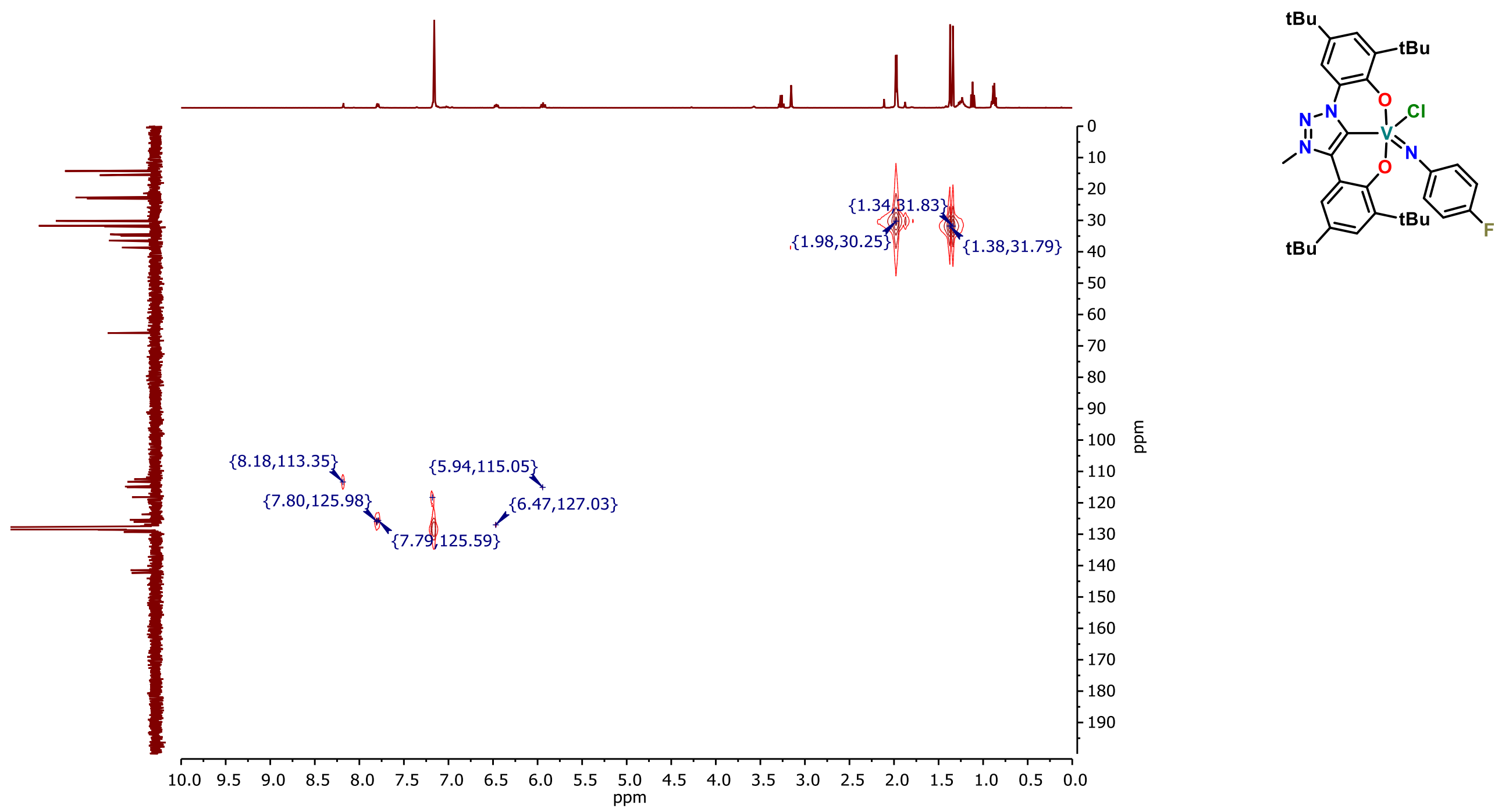

Figure S 37: $\quad{ }^{1} \mathrm{H}^{13} \mathrm{C} H S Q C$ of 8 in $\mathrm{C}_{6} \mathrm{D}_{6}$ 

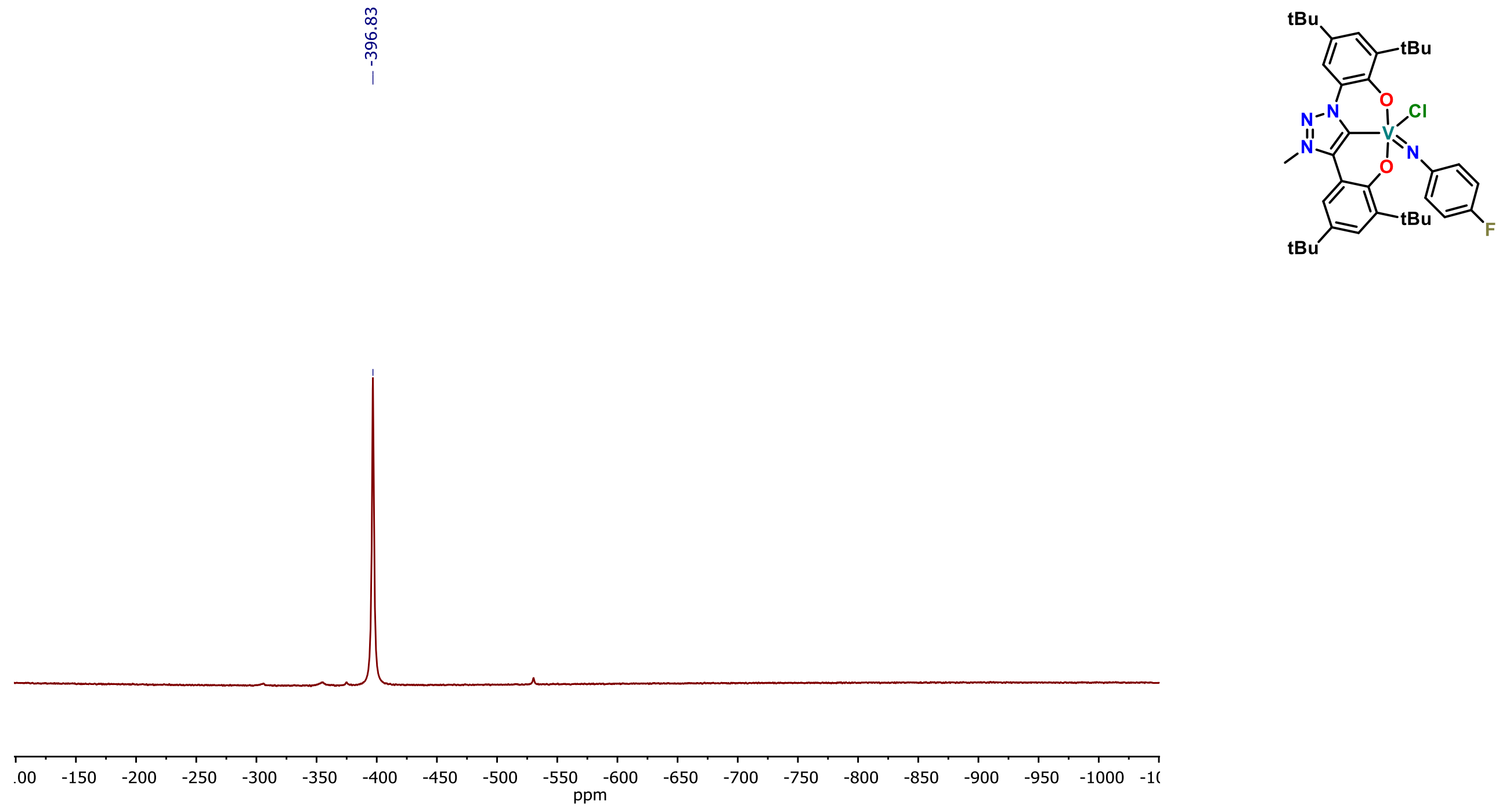

Figure $S$ 38: $\quad{ }^{51} \mathrm{~V}$ NMR of 8 in $\mathrm{C}_{6} \mathrm{D}_{6}$ 

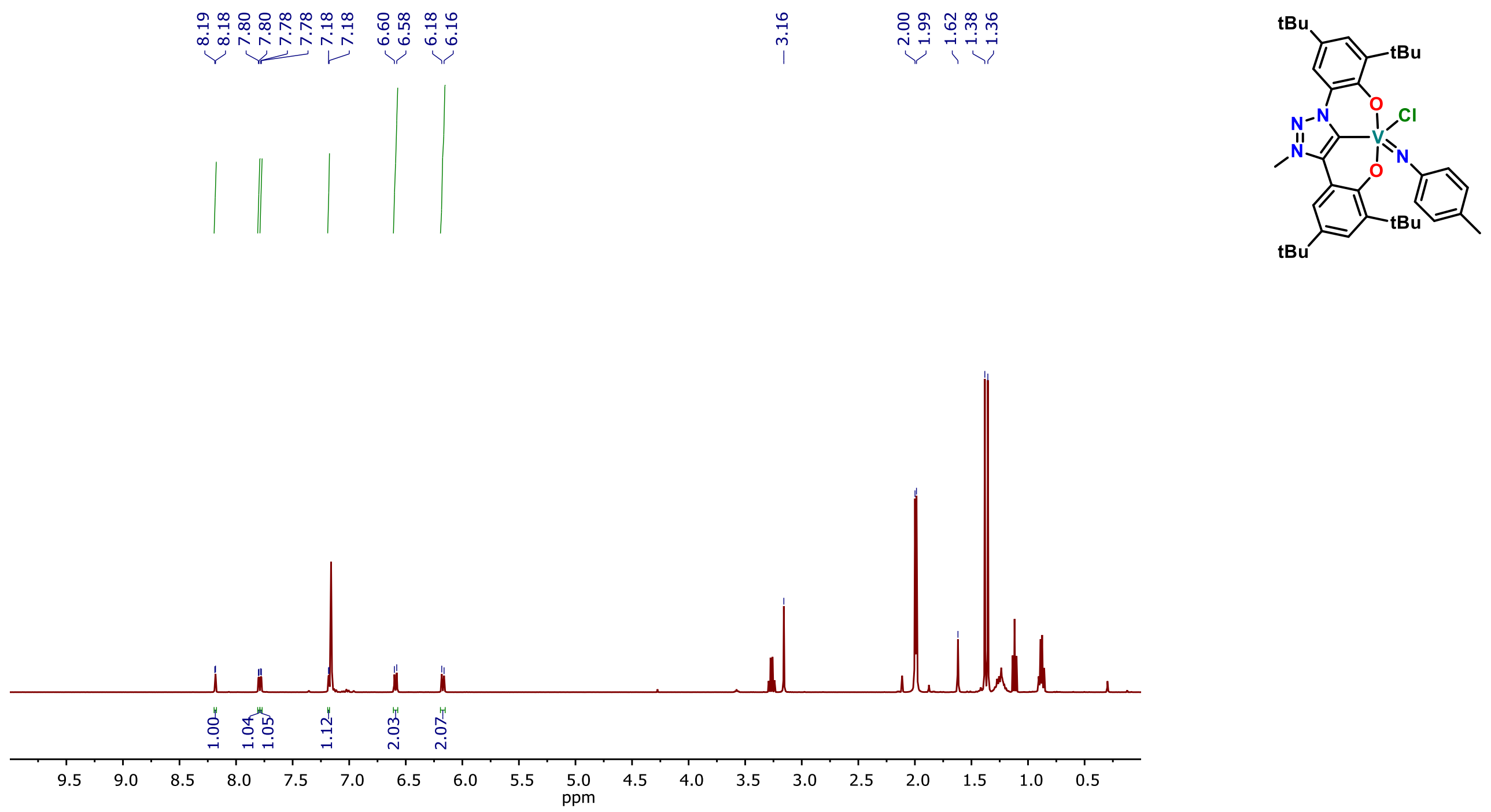

Figure $S$ 39: $\quad{ }^{1} \mathrm{H}$ NMR of 9 in $\mathrm{C}_{6} \mathrm{D}_{6}$ 


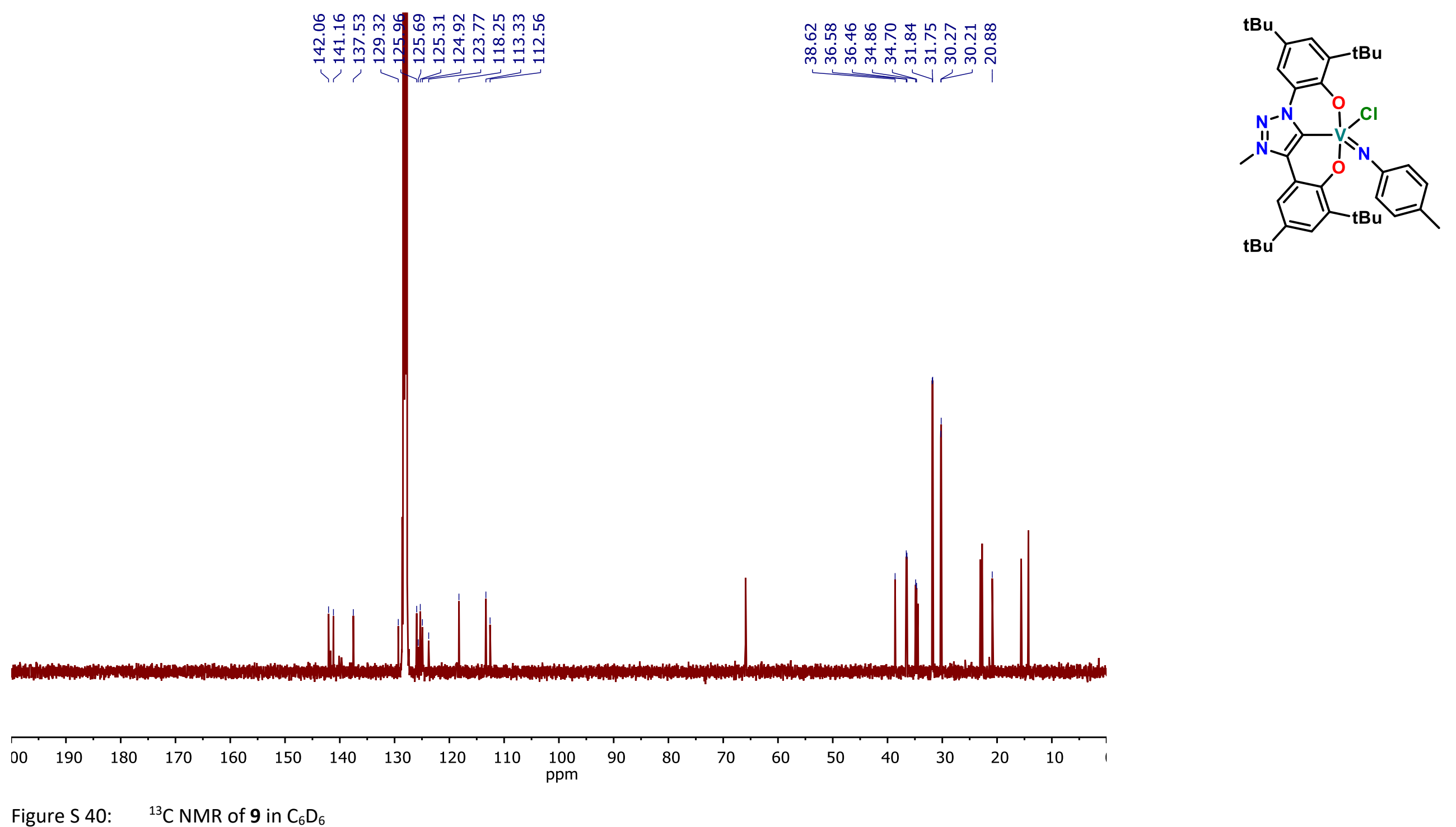



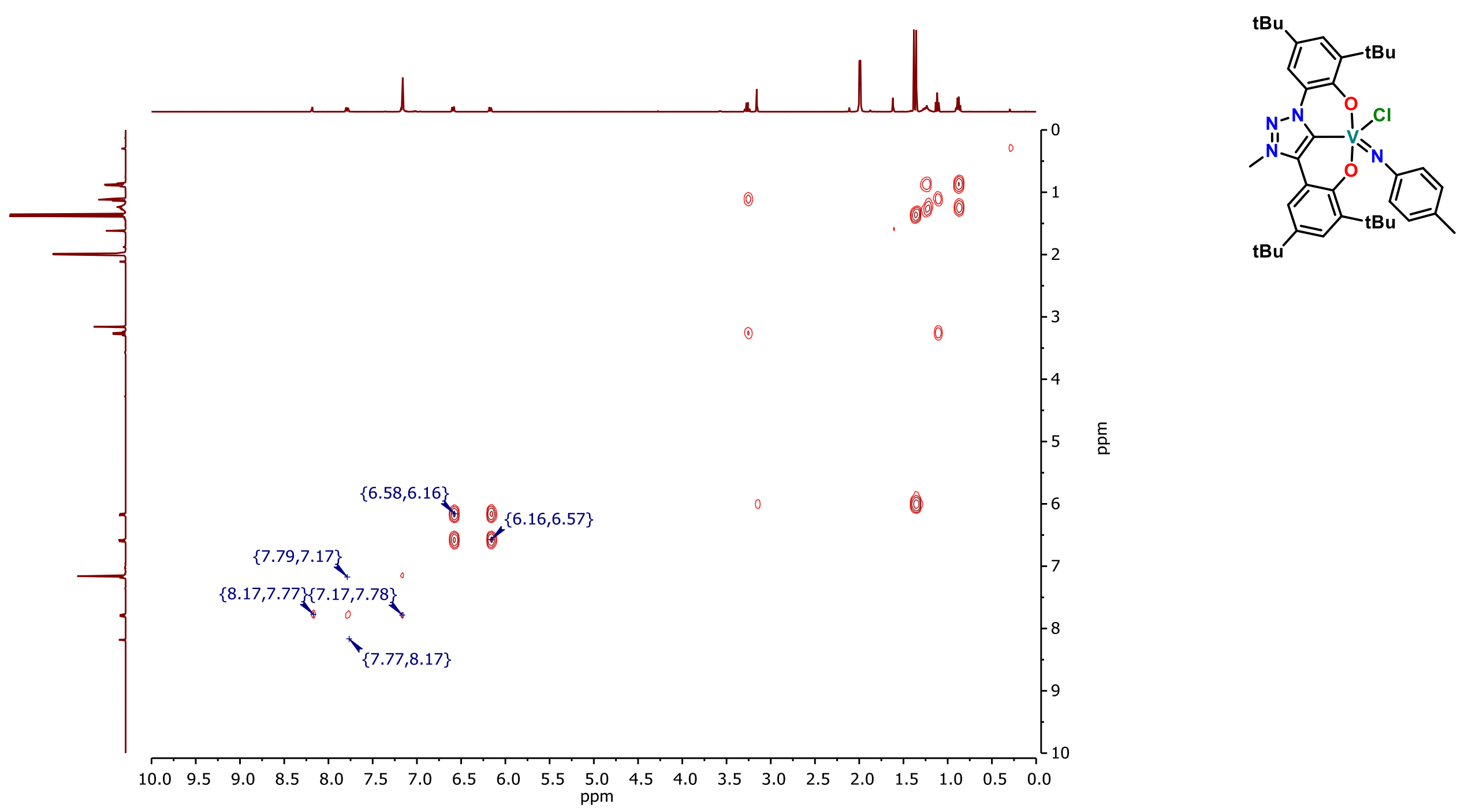

Figure $\mathrm{S} 41: \quad{ }^{1} \mathrm{H}^{1} \mathrm{H}$ COSY of 9 in $\mathrm{C}_{6} \mathrm{D}_{6}$ 

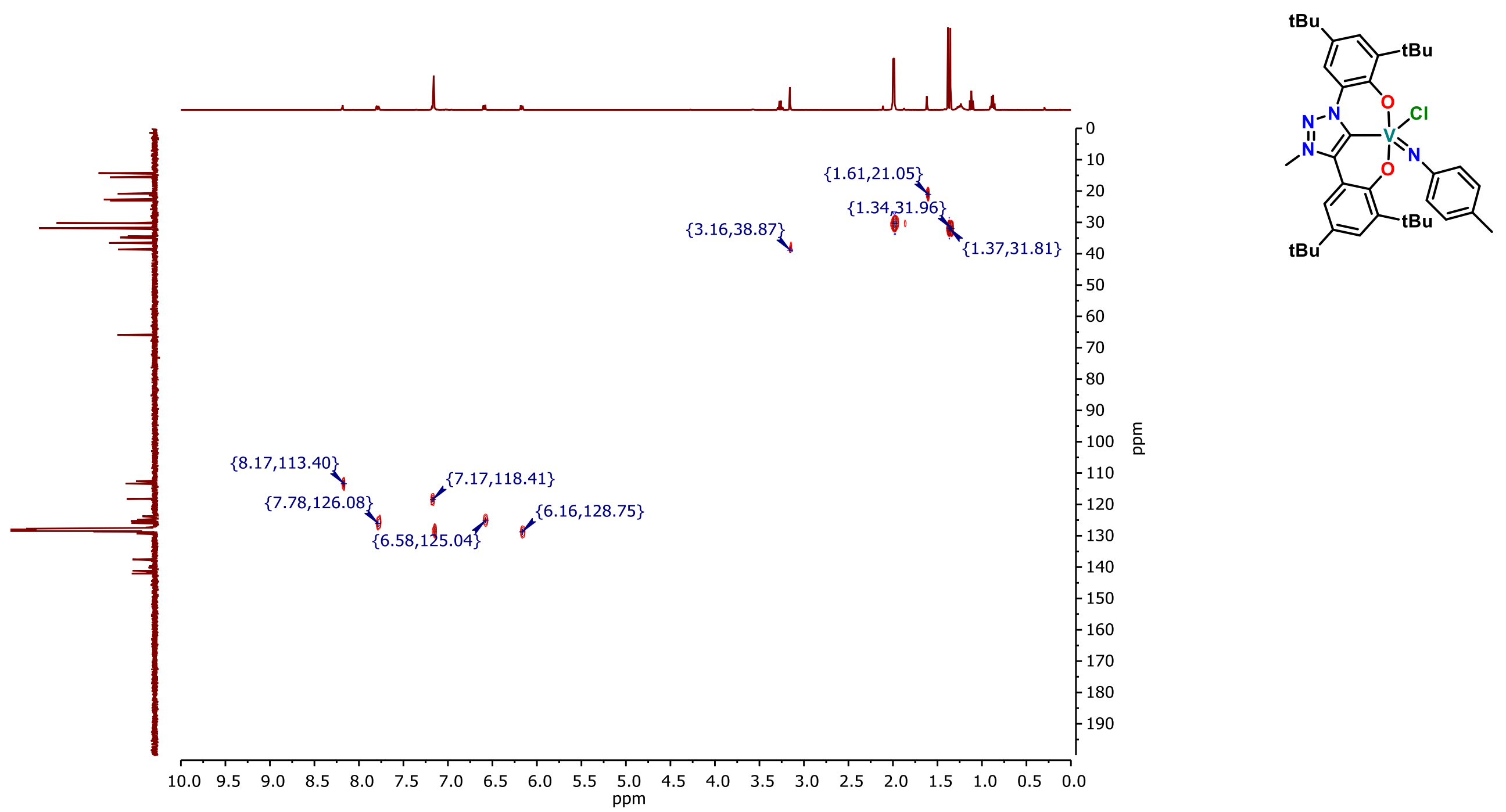

Figure $S 42: \quad{ }^{1} H^{13} \mathrm{C}$ HSQC of 9 in $C_{6} D_{6}$ 

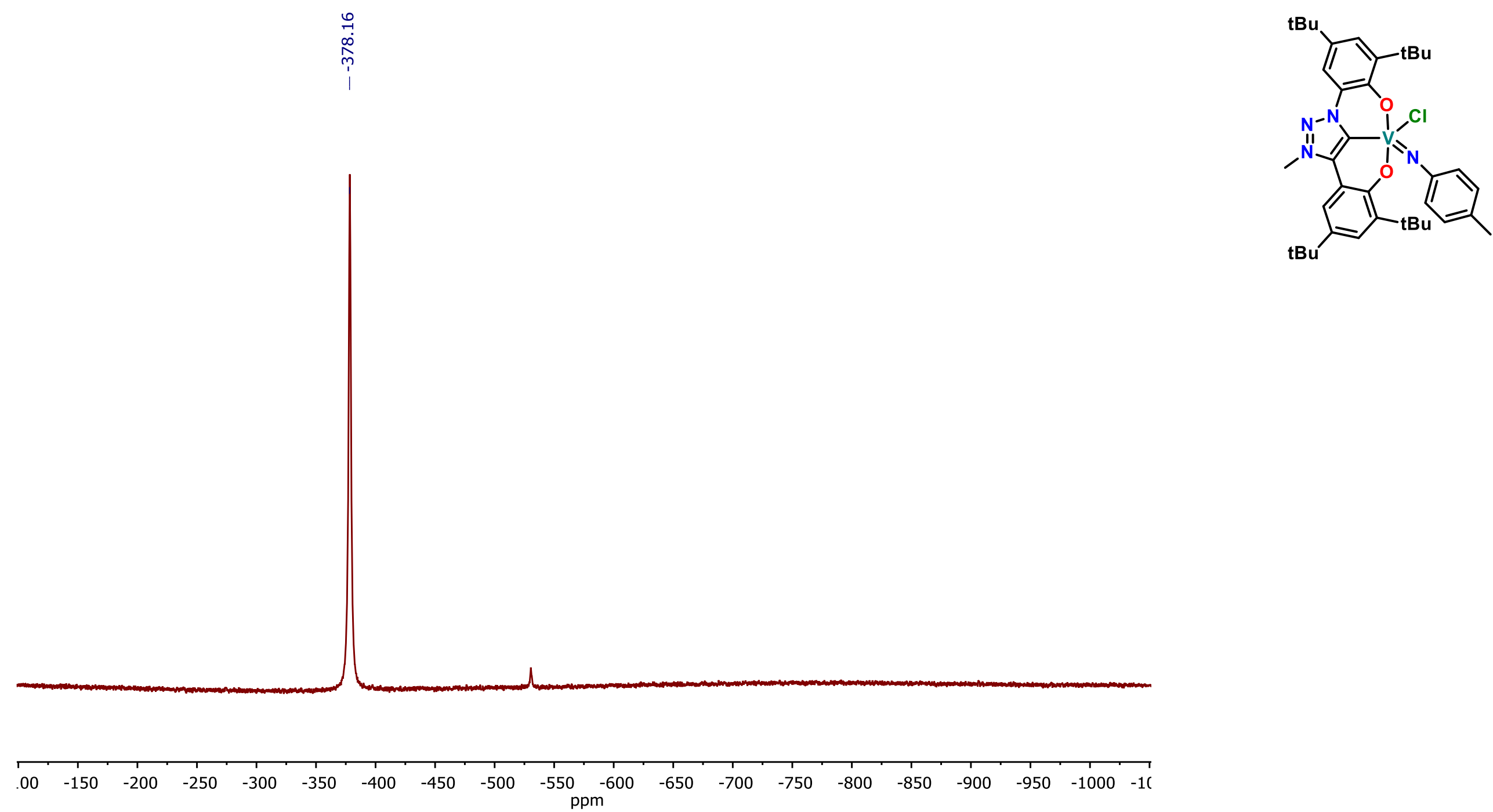

Figure $S 43: \quad{ }^{51} \mathrm{~V}$ NMR of 9 in $\mathrm{C}_{6} \mathrm{D}_{6}$ 

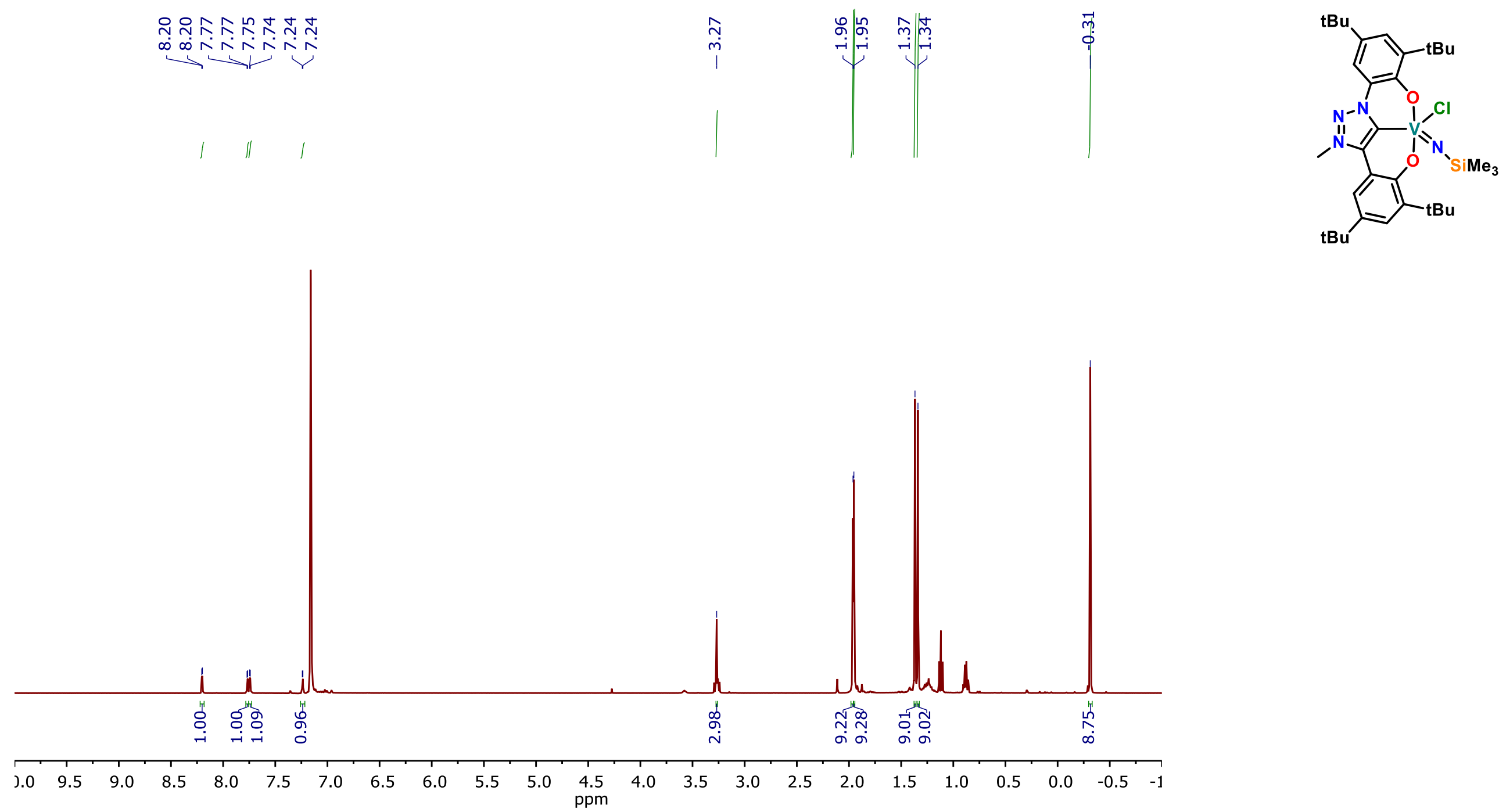

Figure $S 44: \quad{ }^{1} \mathrm{H}$ NMR of 10 in $C_{6} D_{6}$ 


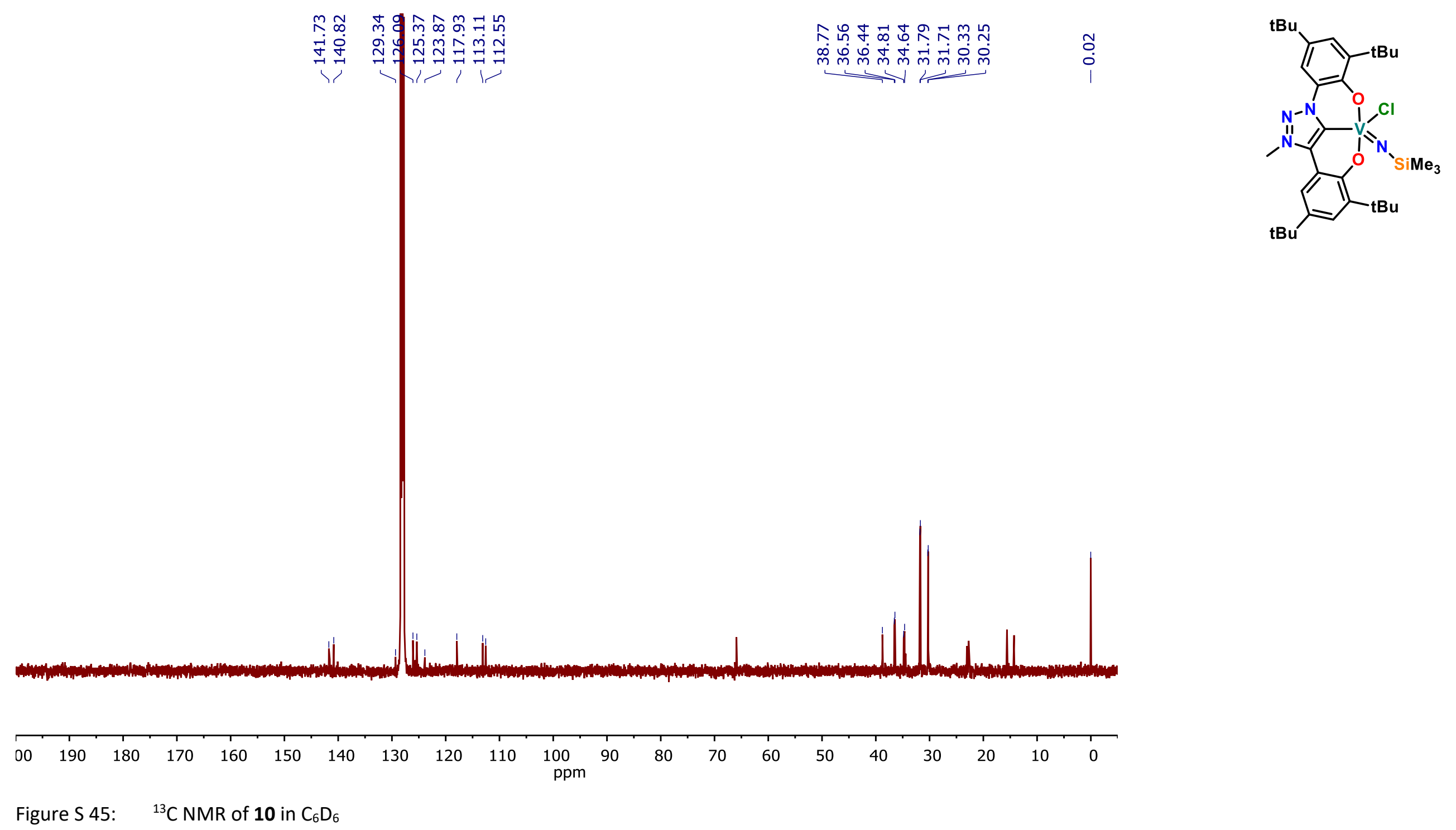




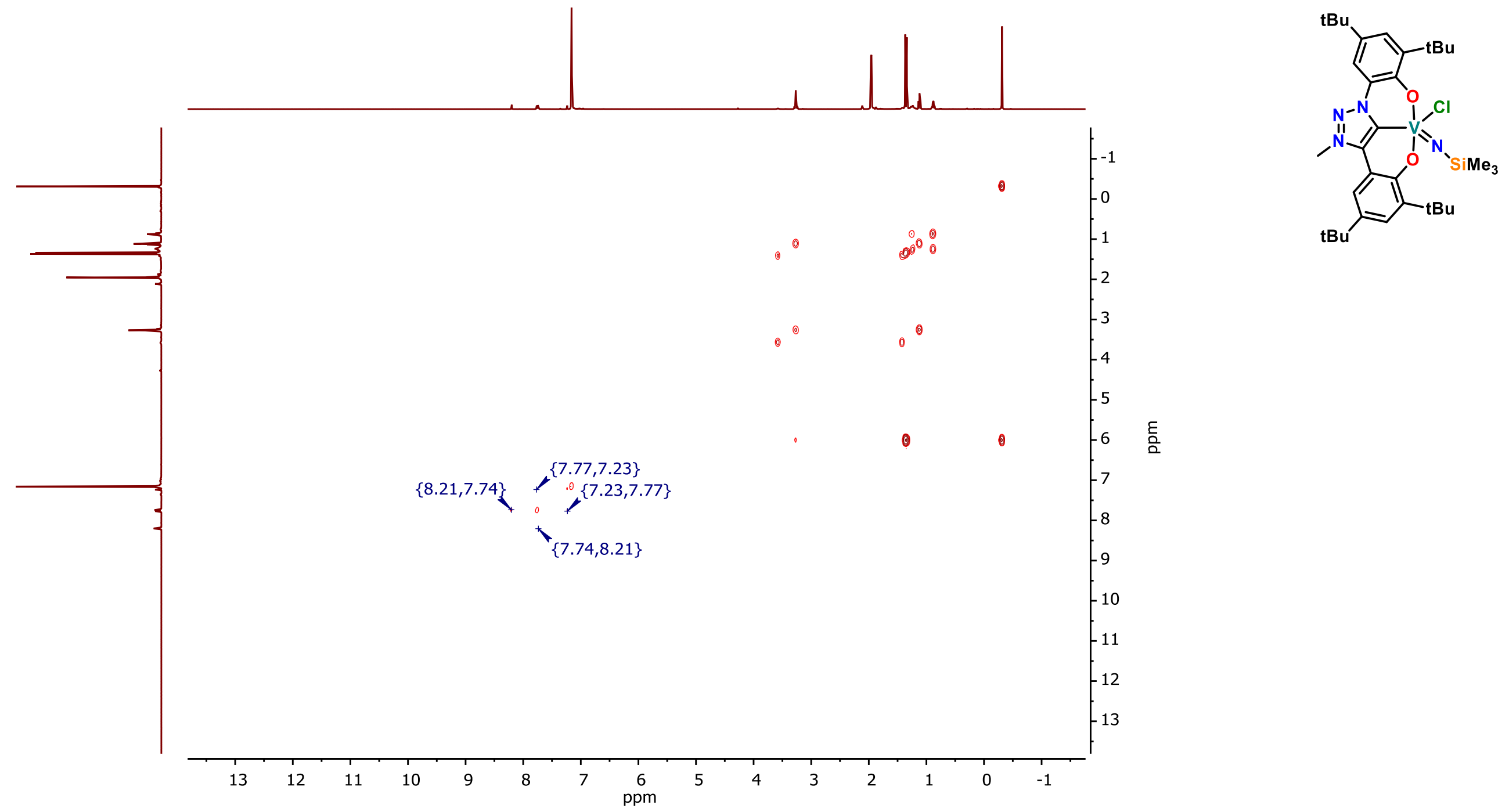

Figure $S$ 46: $\quad{ }^{1} \mathrm{H}^{1} \mathrm{H}$ COSY of 10 in $\mathrm{C}_{6} \mathrm{D}_{6}$. Cross peaks in the aliphatic region arise from solvent impurities (diethyl ether, pentane and THF as well as from large T1 noise from the ${ }^{t} \mathrm{Bu}$ and $\mathrm{SiMe}_{3}$ groups. 

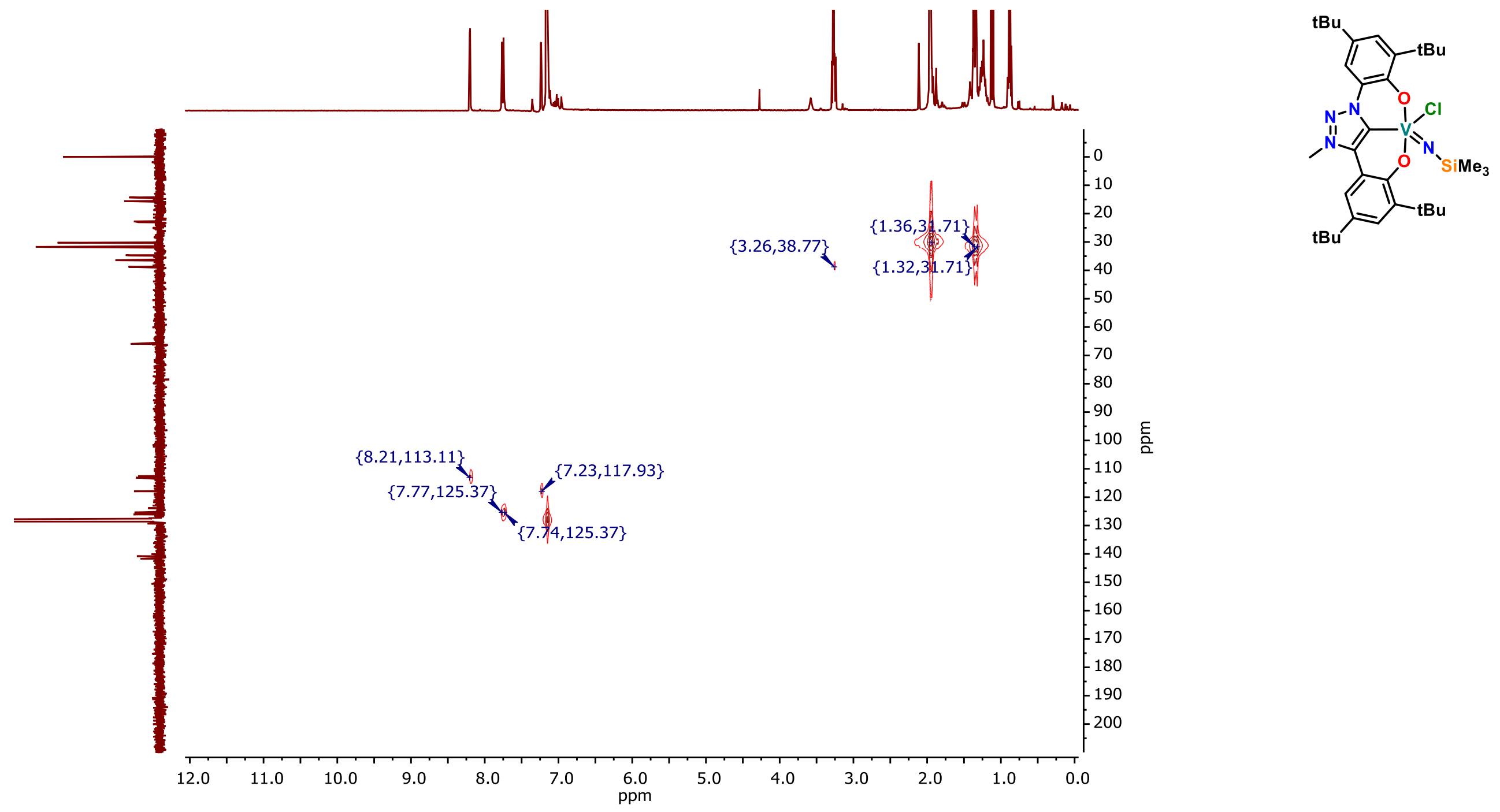

Figure $\mathrm{S} 47: \quad{ }^{1} \mathrm{H}^{13} \mathrm{C}$ HSQC of $\mathbf{1 0}$ in $\mathrm{C}_{6} \mathrm{D}_{6}$ 

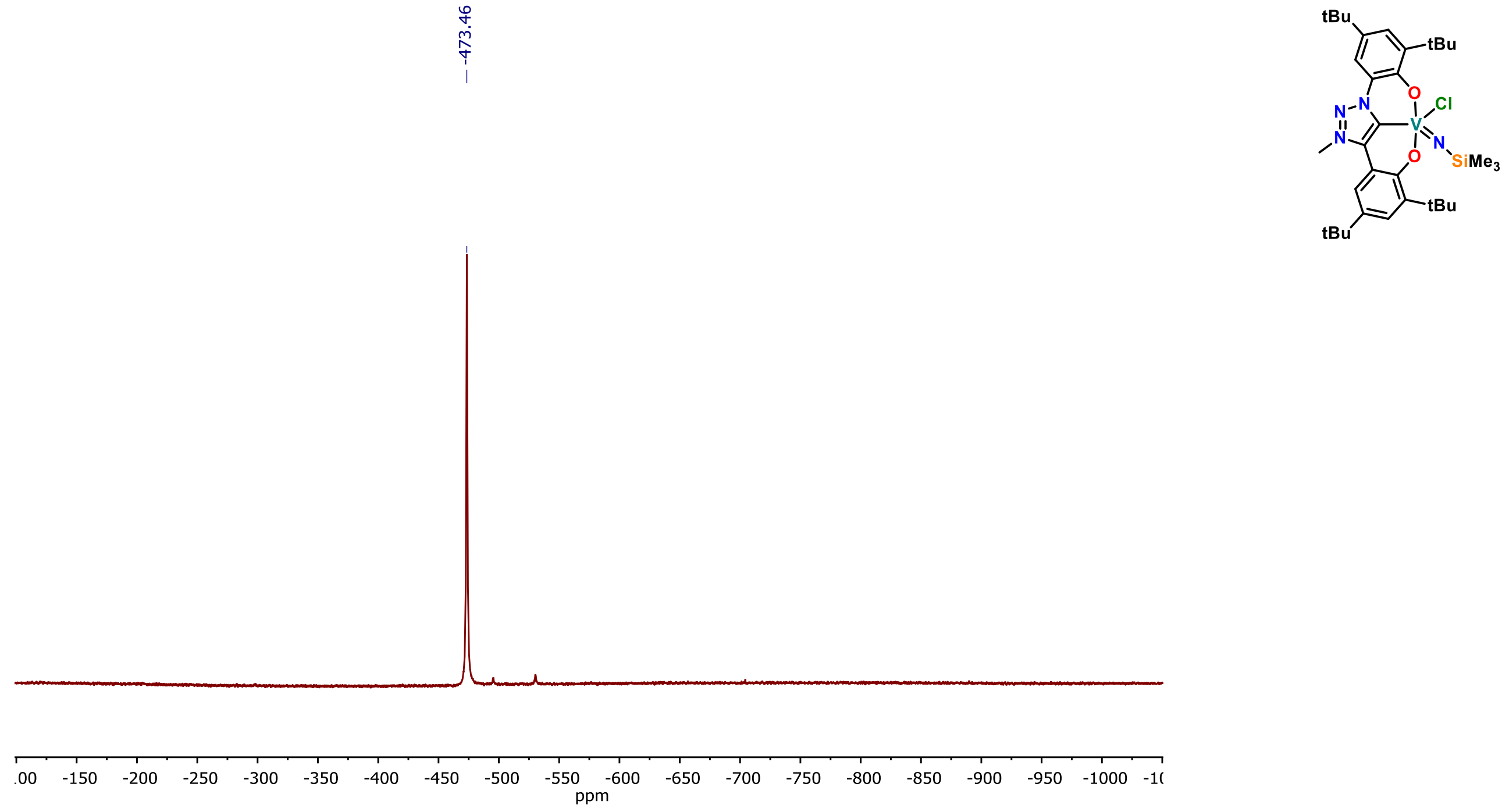

Figure $S 48: \quad{ }^{51} \mathrm{~V}$ NMR of 10 in $\mathrm{C}_{6} \mathrm{D}_{6}$ 


\section{IR spectroscopy}

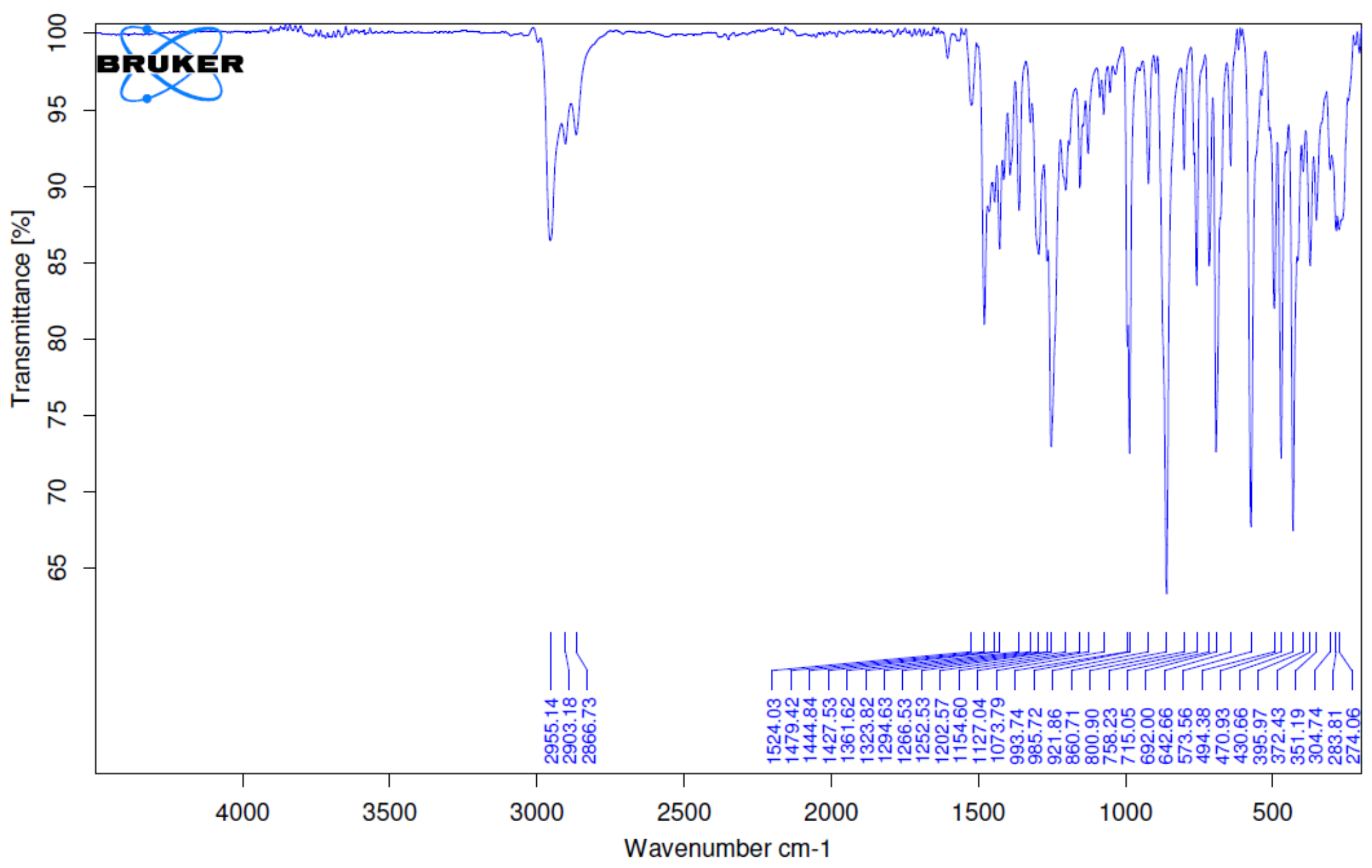

$\mathrm{tBu}$

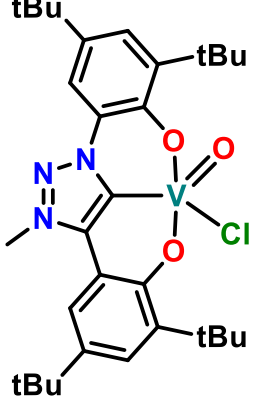

Figure S 49: $\quad$ ATR-IR spectrum of 1 at 298 K. 

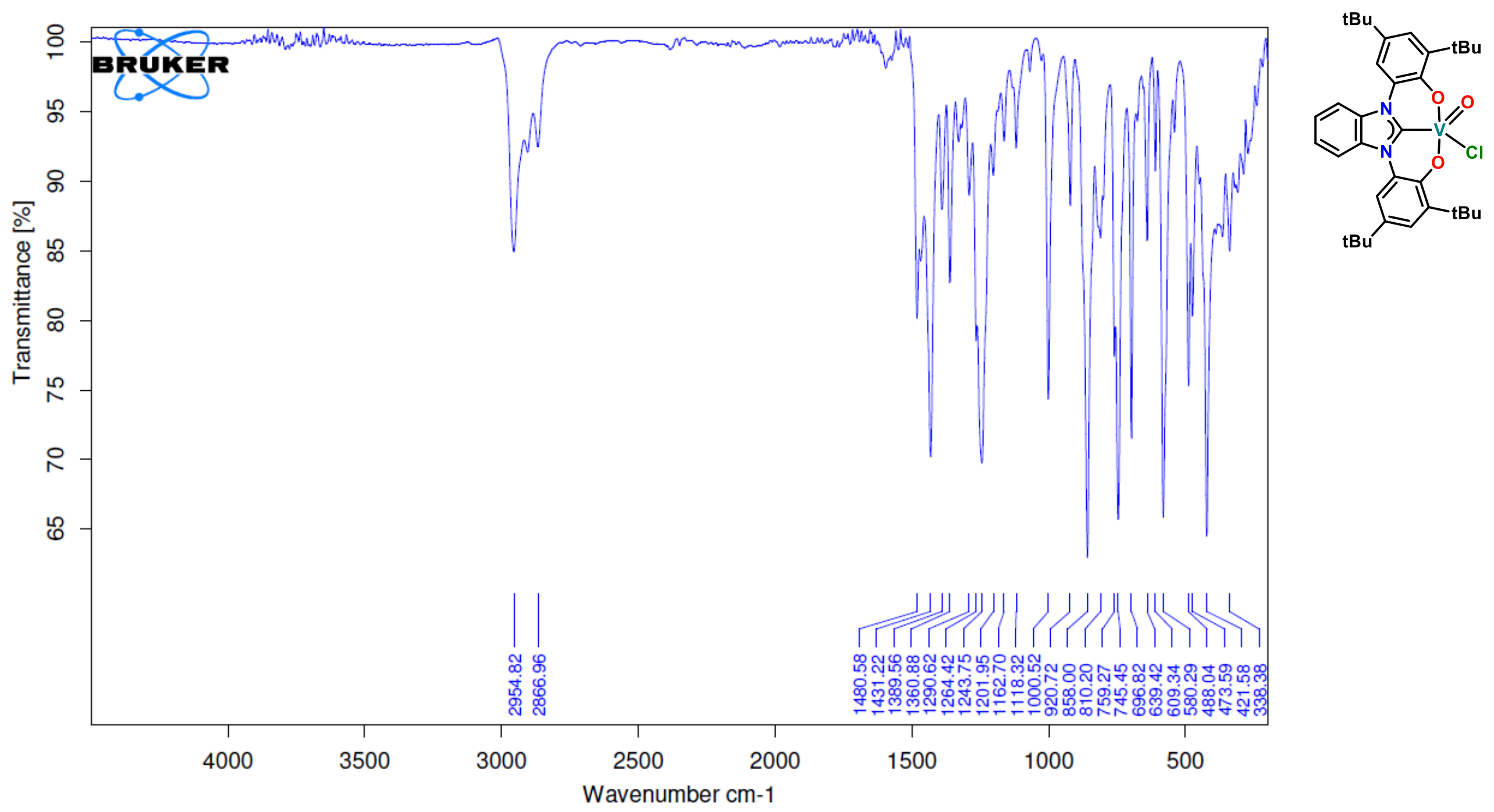

Figure S 50: $\quad$ ATR-IR spectrum of $\mathbf{2}$ at $298 \mathrm{~K}$. 

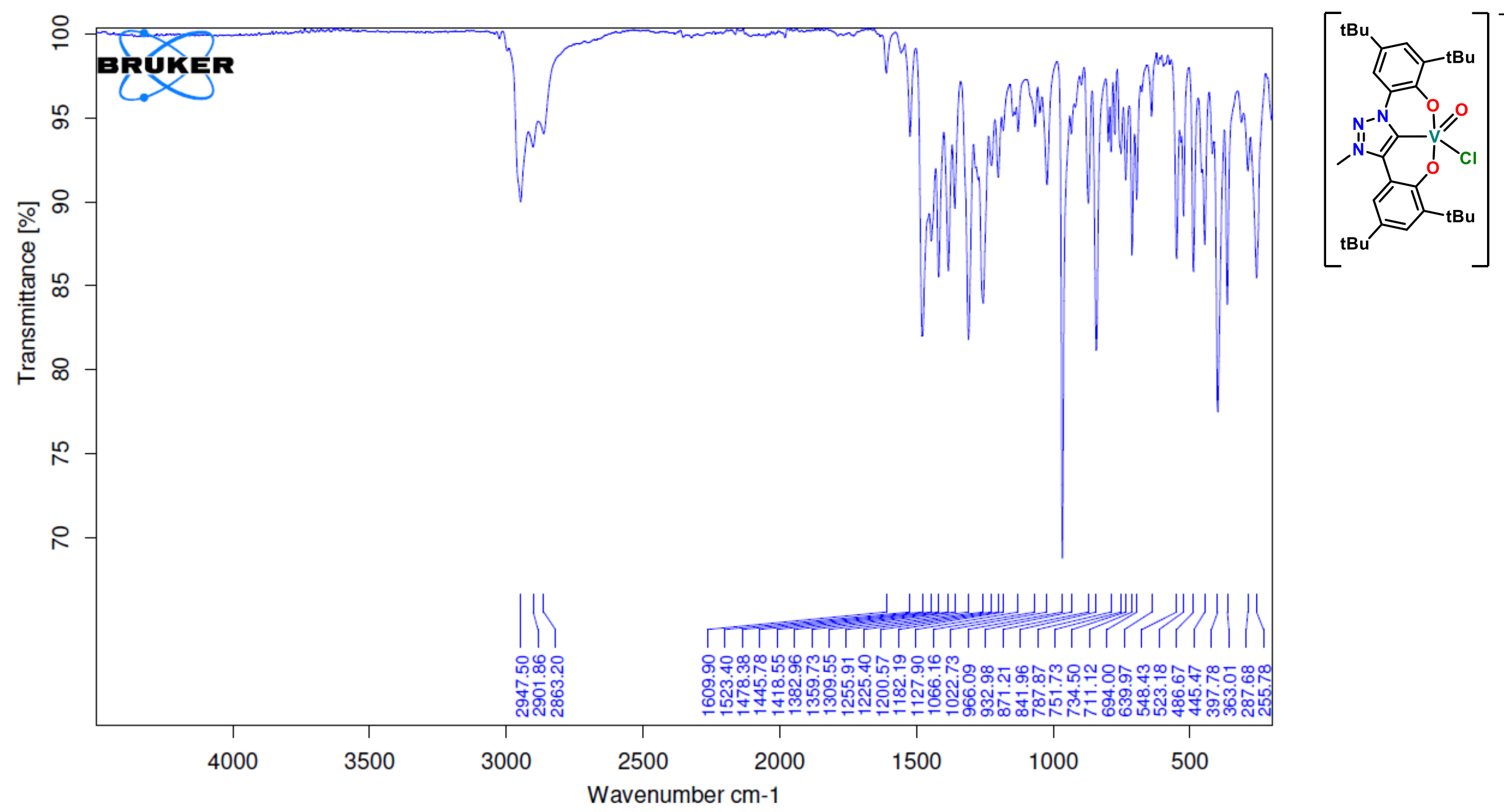

Figure S 51: $\quad$ ATR-IR spectrum of $\left[\mathrm{Co}\left(\mathrm{Cp}^{*}\right)_{2}\right][1]$ at $298 \mathrm{~K}$. 


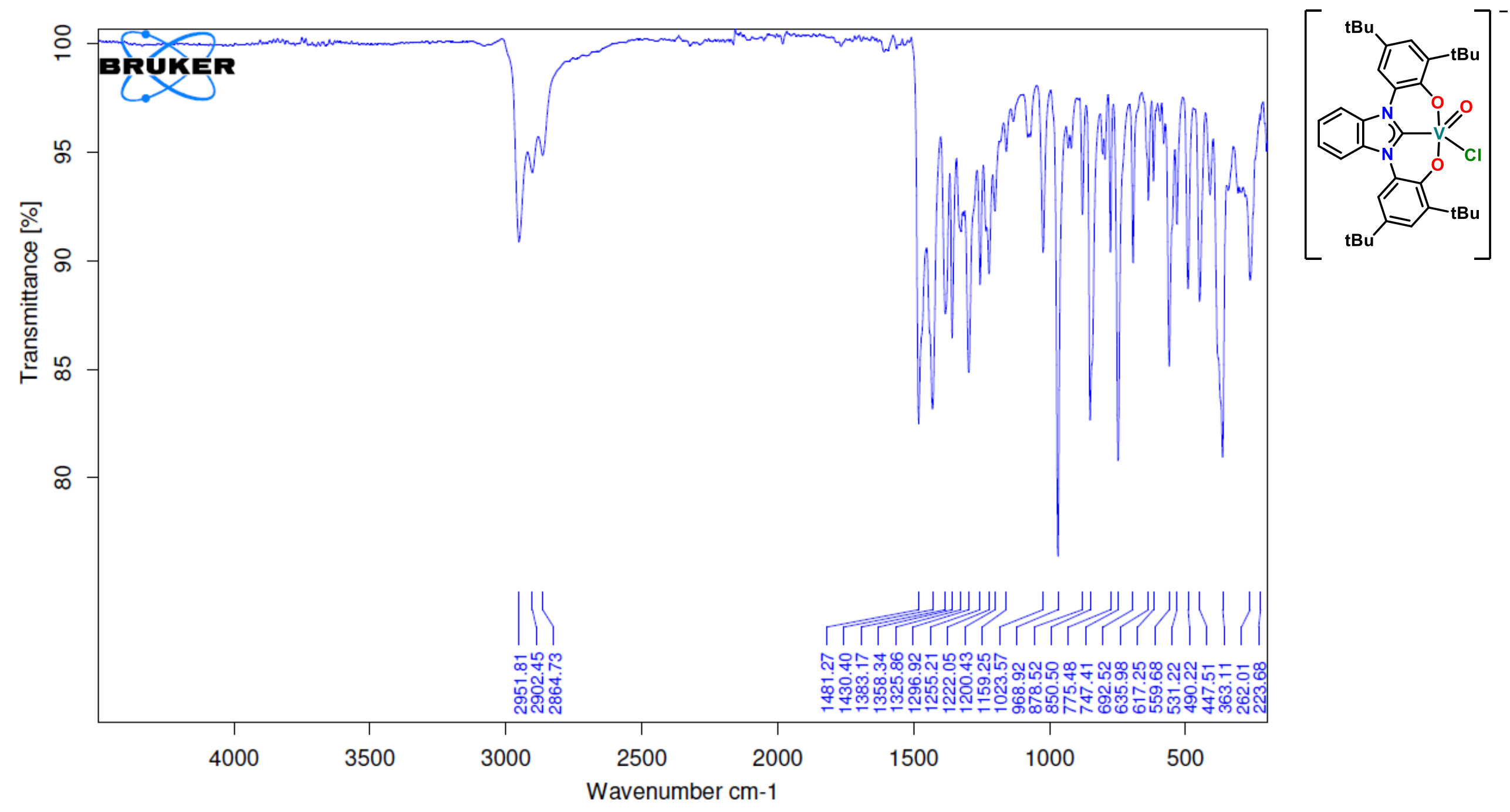

Figure S 52: ATR-IR spectrum of $\left[\mathrm{Co}\left(\mathrm{Cp}^{*}\right)_{2}\right][2]$ at $298 \mathrm{~K}$. 

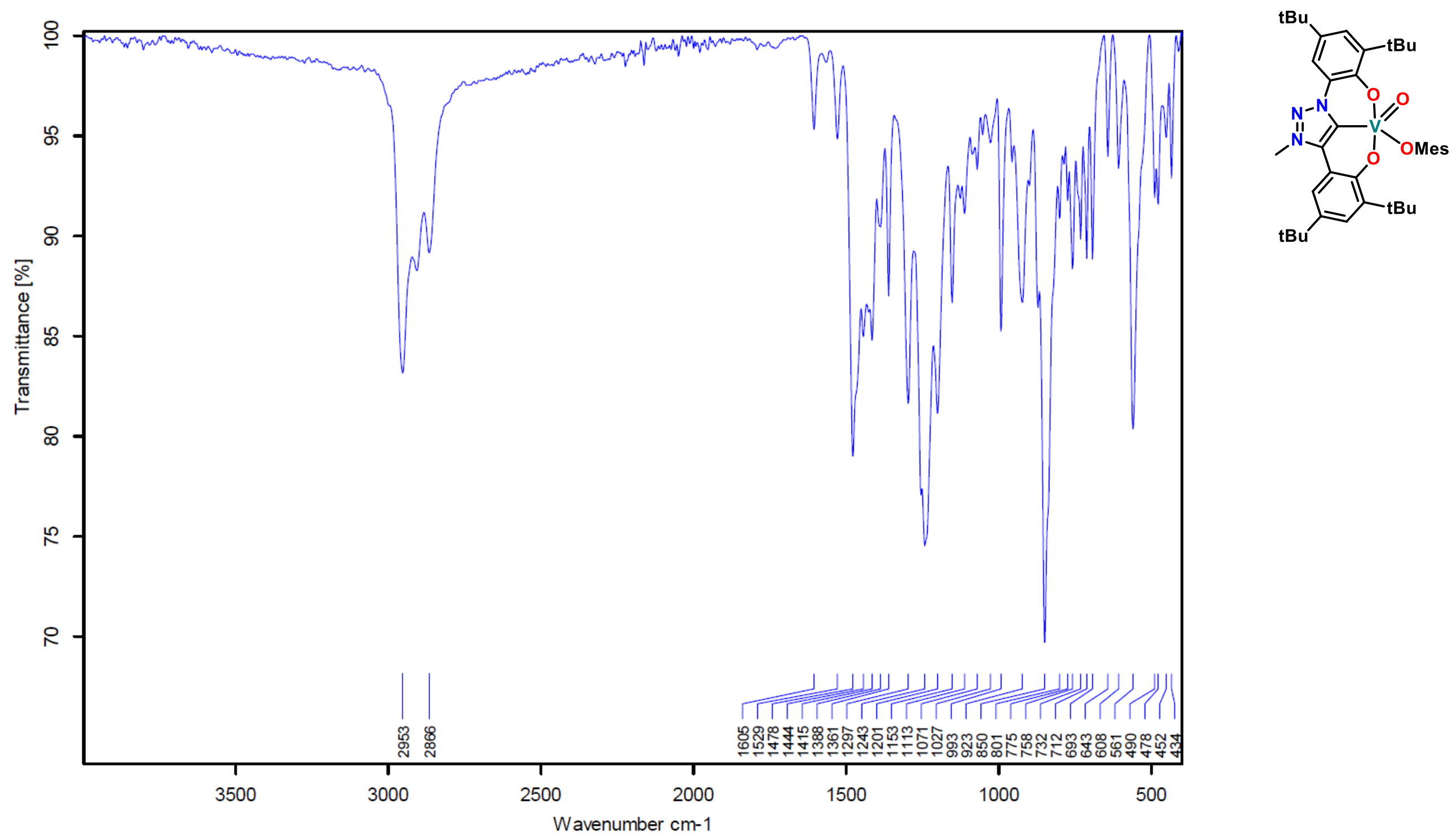

Figure S 53: ATR-IR spectrum of 3 at 298K. 

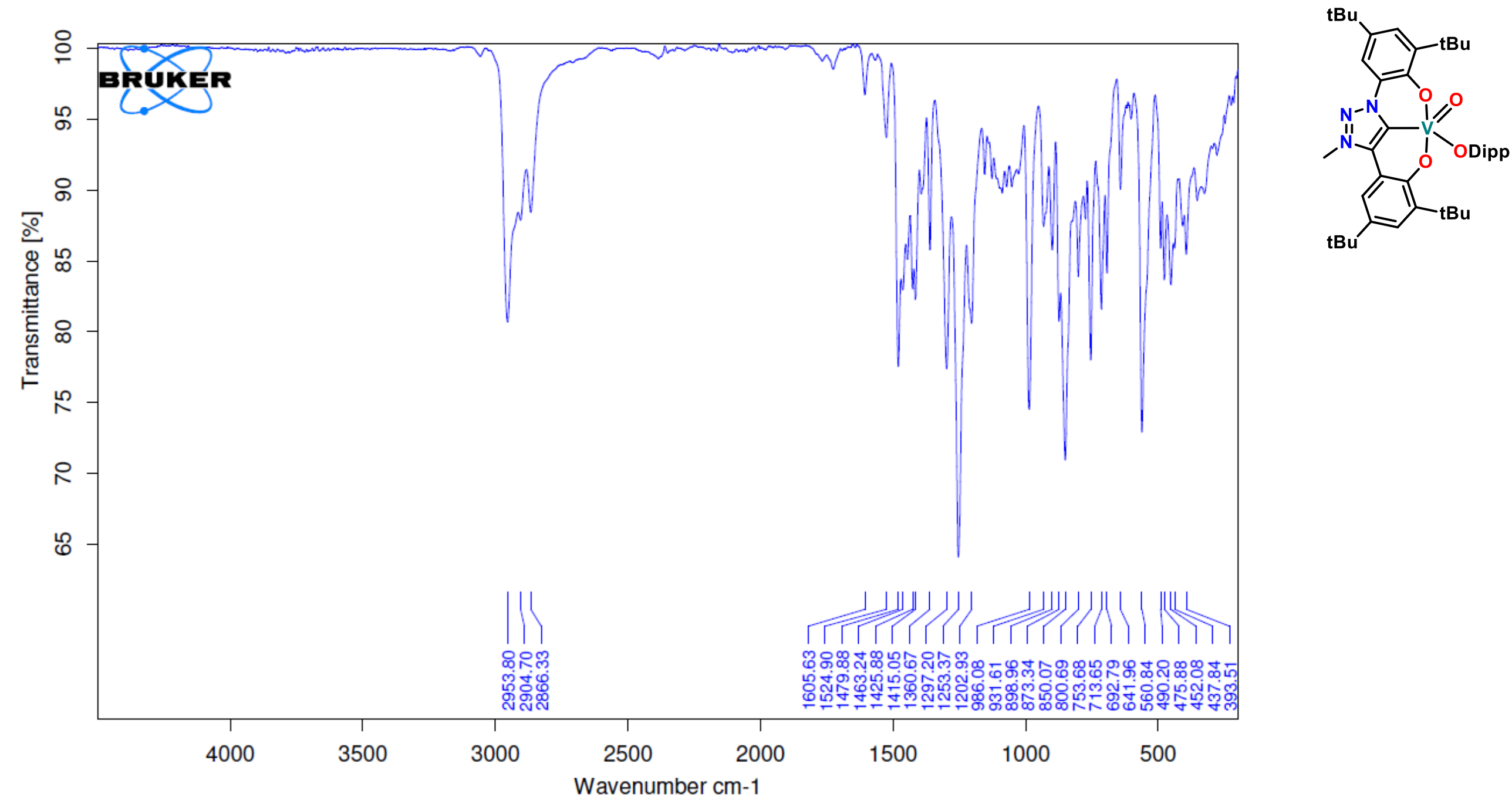

Figure S 54: $\quad$ ATR-IR spectrum of 4 at $298 \mathrm{~K}$. 

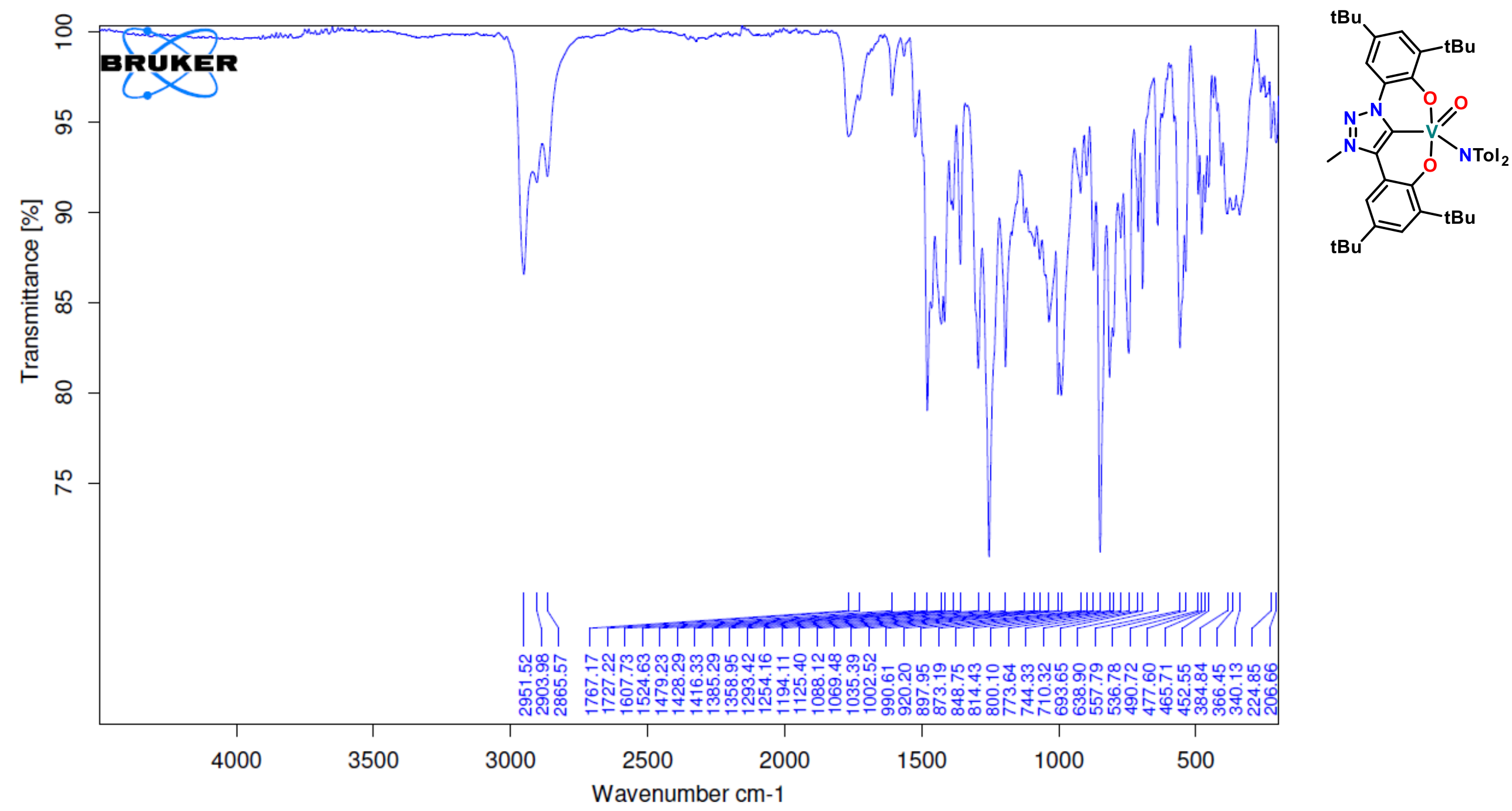

Figure S 55: $\quad$ ATR-IR spectrum of 5 at 298K. 

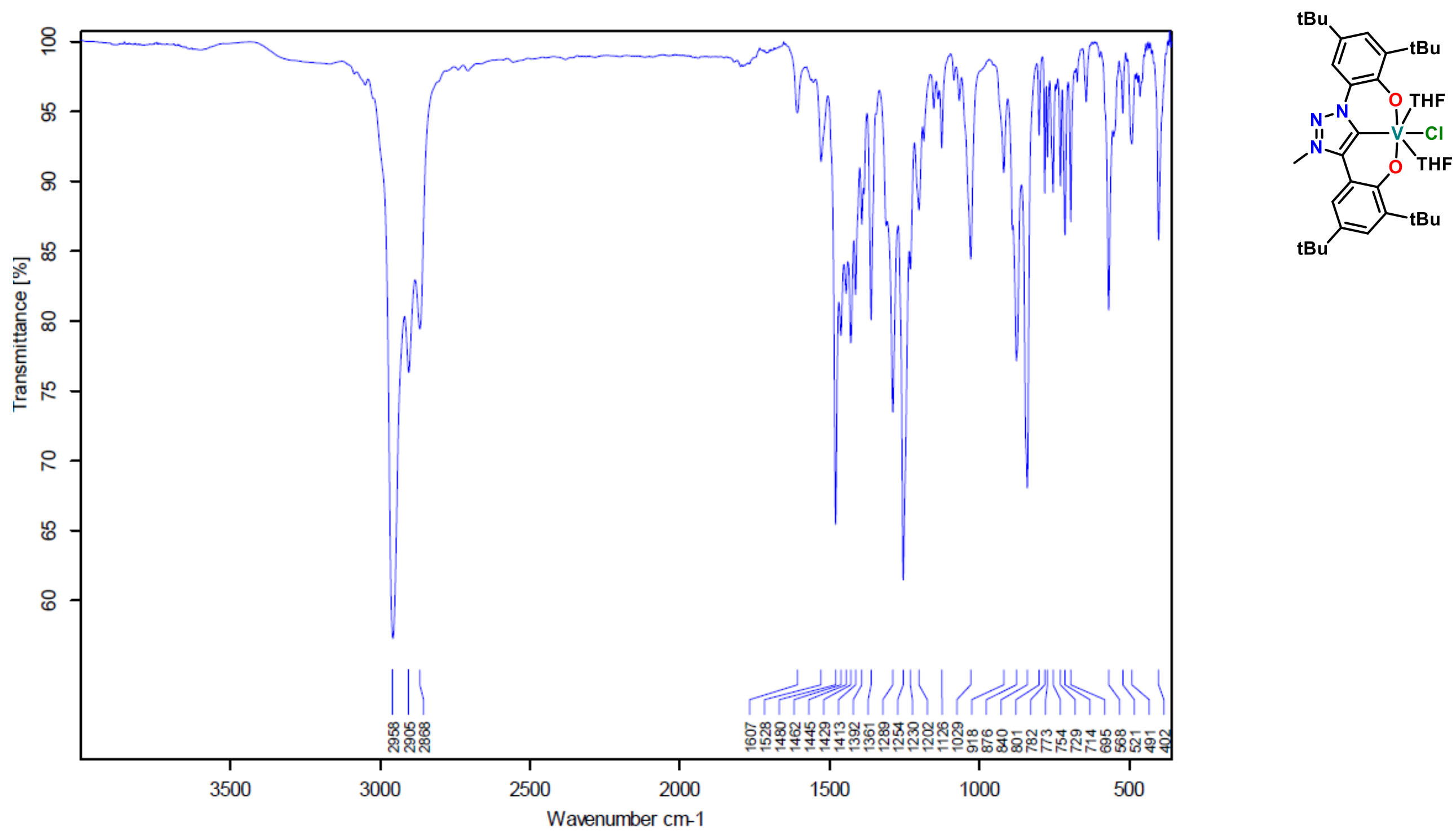

Figure S 56: $\quad$ ATR-IR spectrum of 6 at 298K 

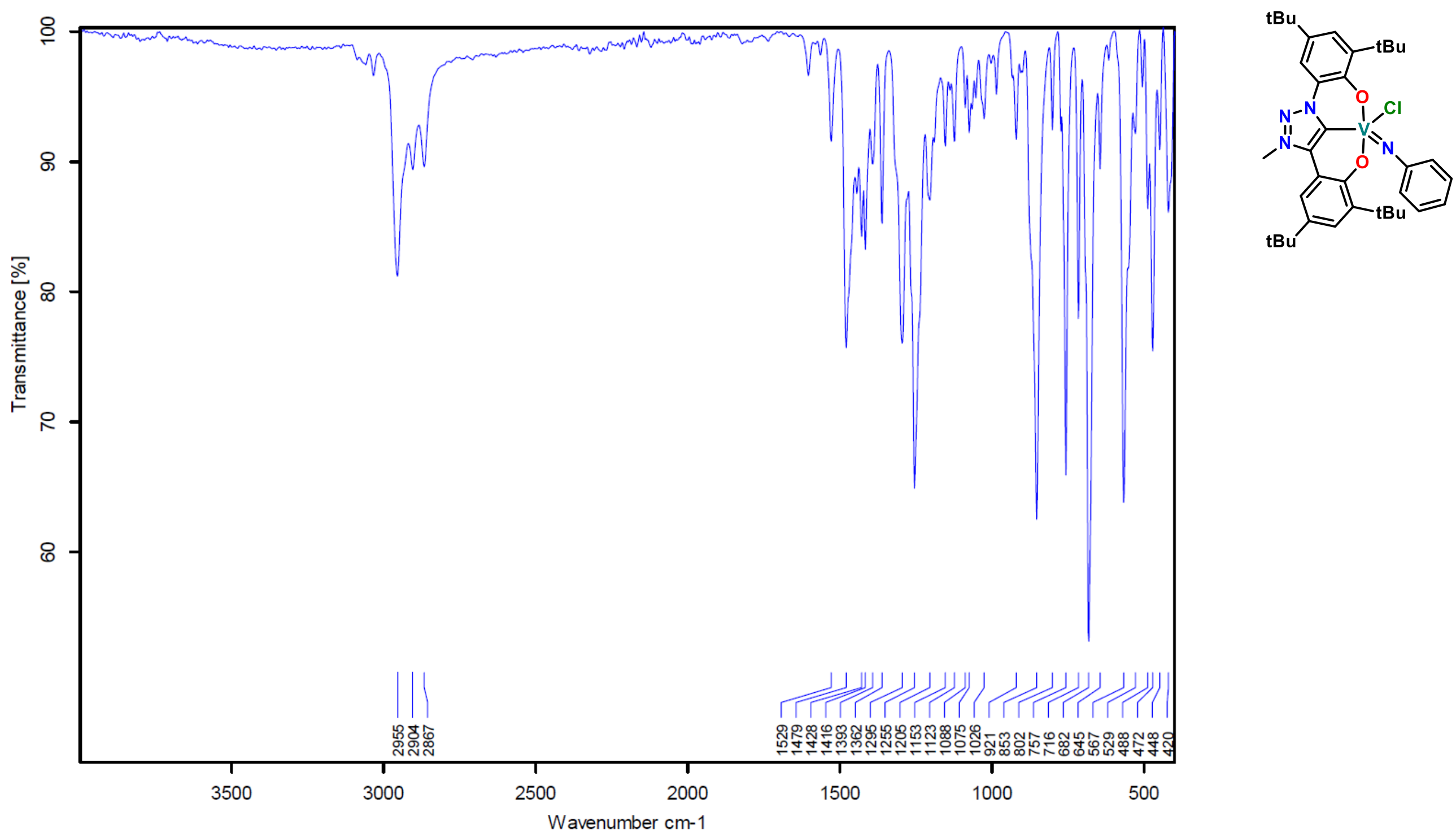

Figure S 57: $\quad$ ATR-IR spectrum of 7 at $298 \mathrm{~K}$ 

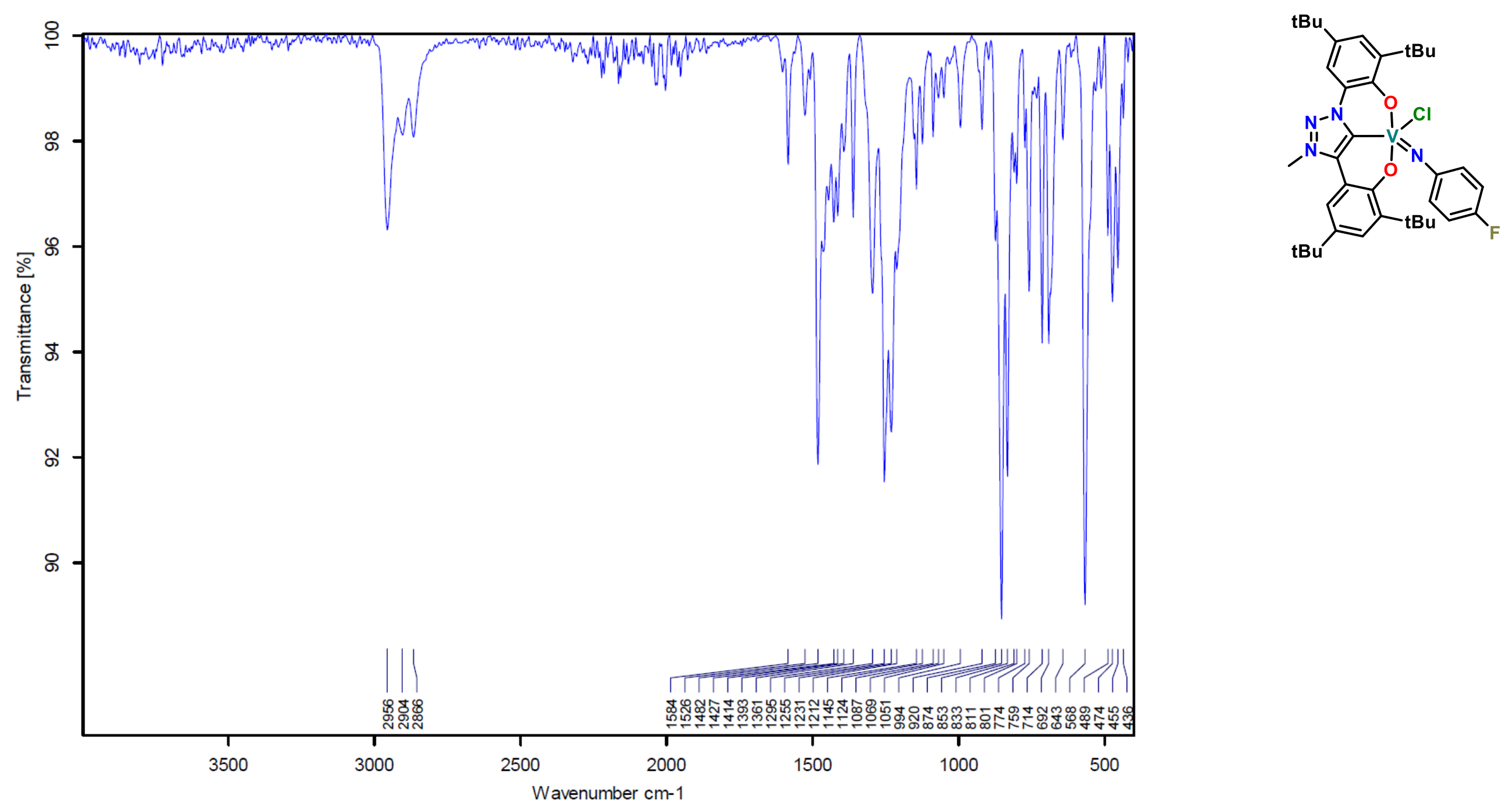

Figure S 58: $\quad$ ATR-IR spectrum of 8 at $298 \mathrm{~K}$ 

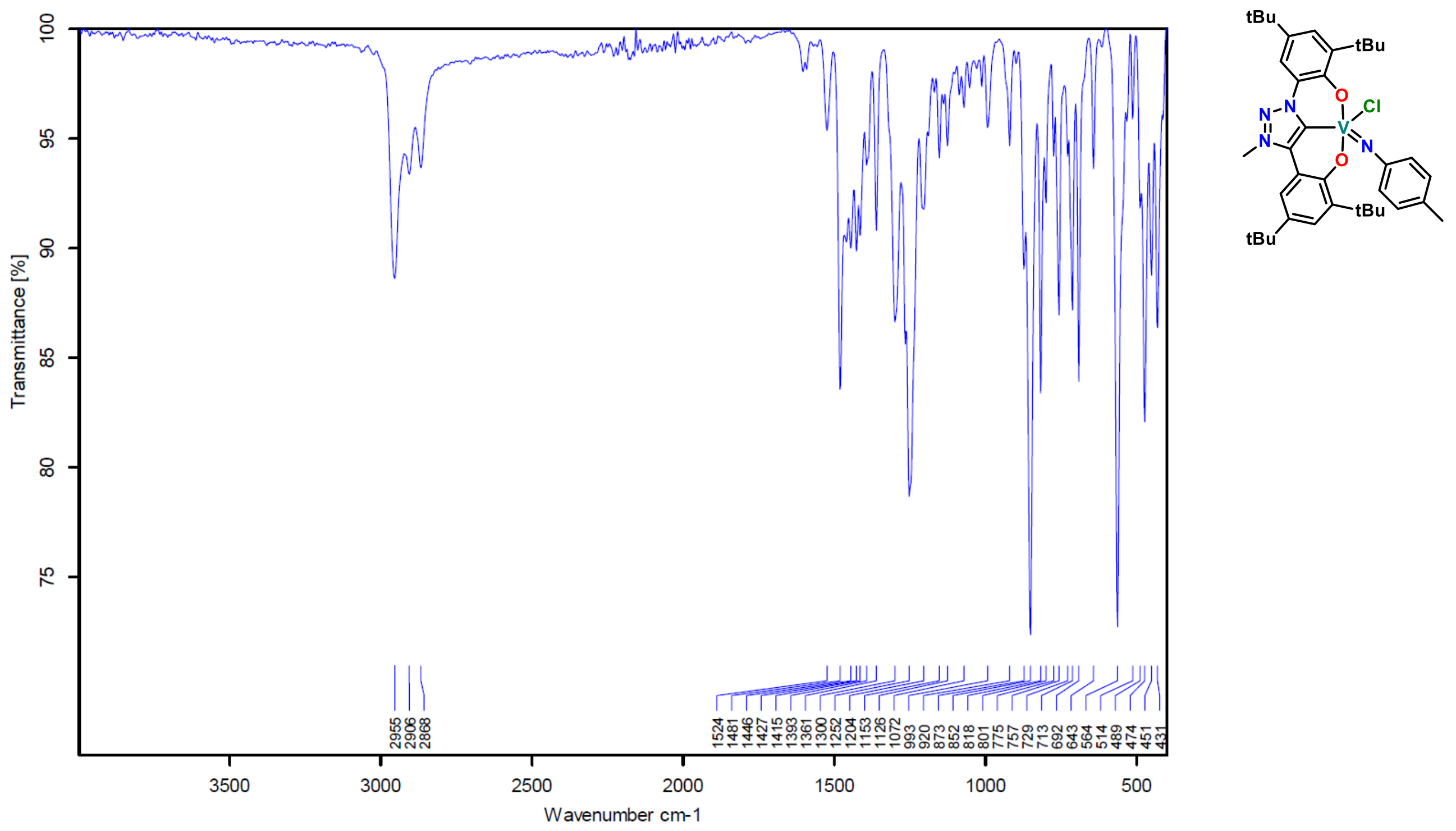

Figure S 59: $\quad$ ATR-IR spectrum of 9 at $298 \mathrm{~K}$ 

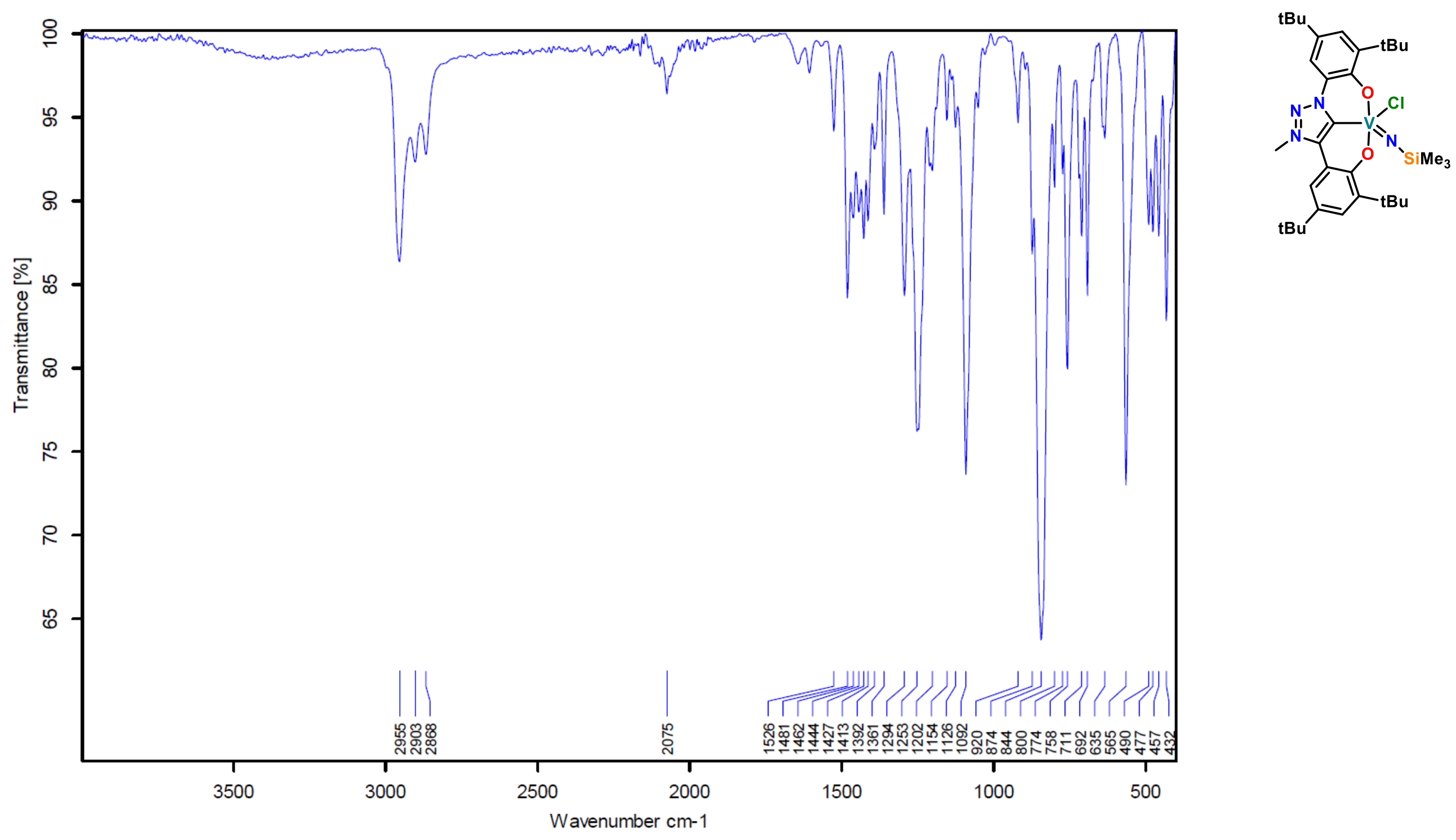

Figure S 60: $\quad$ ATR-IR spectrum of 10 at $298 \mathrm{~K}$ 
3. UV-Vis spectra

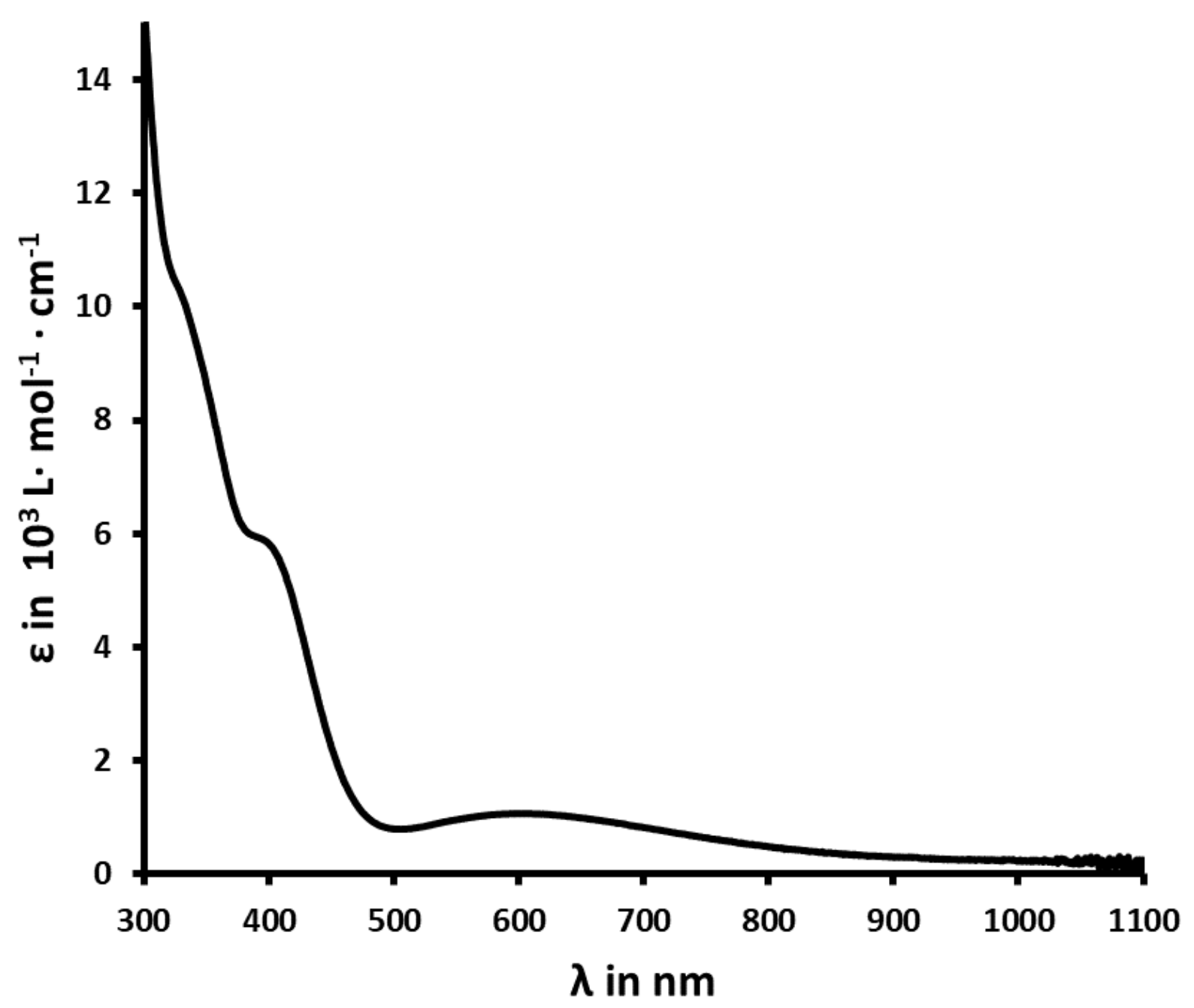

Figure S 61: UV-Vis of 1 in $\mathrm{CH}_{2} \mathrm{Cl}_{2}$ at 298K. 


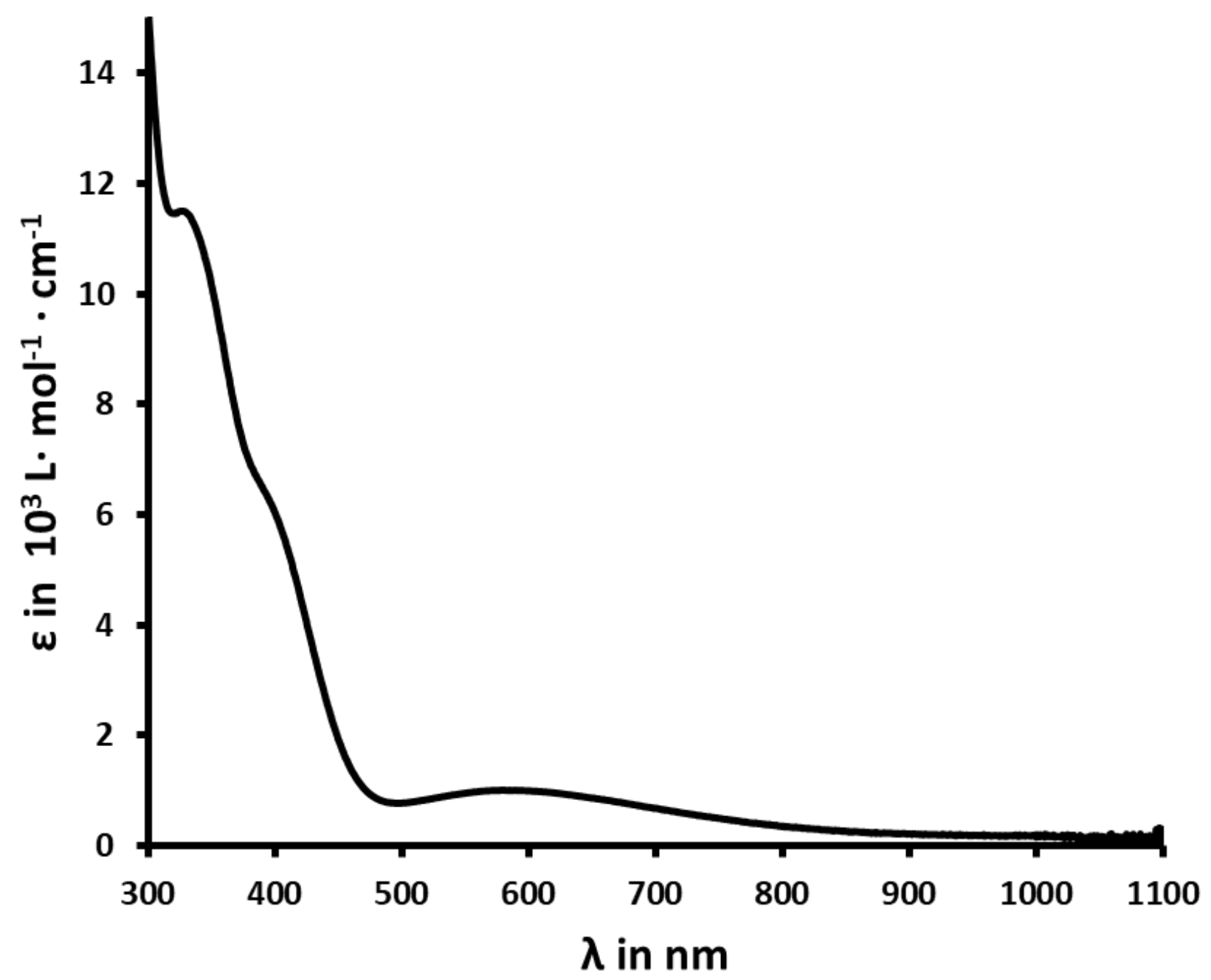

Figure S 62: UV-Vis of 1 in THF at 298K. 


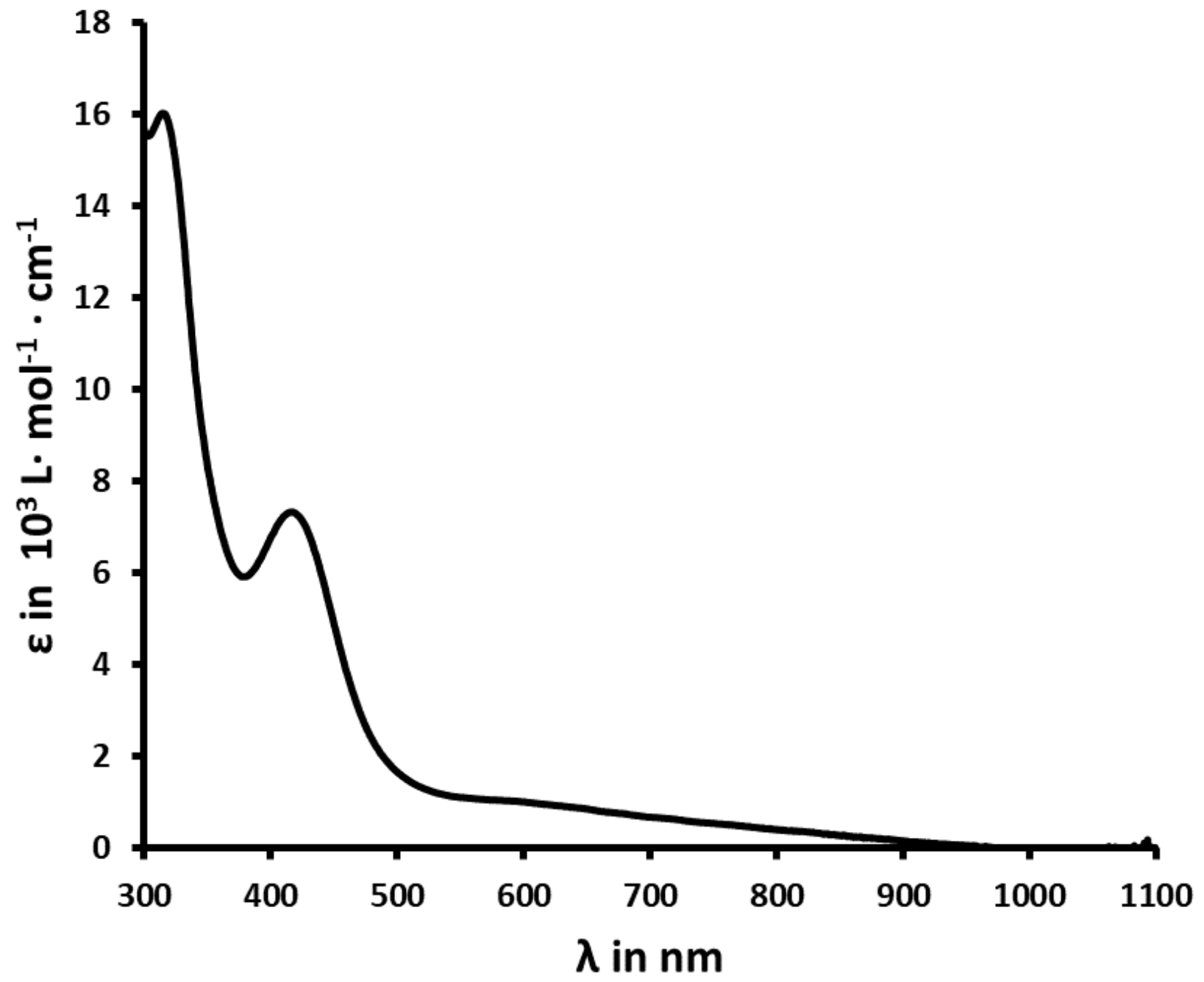

Figure S 63: UV-Vis of 2 in $\mathrm{CH}_{2} \mathrm{Cl}_{2}$ at $298 \mathrm{~K}$. 


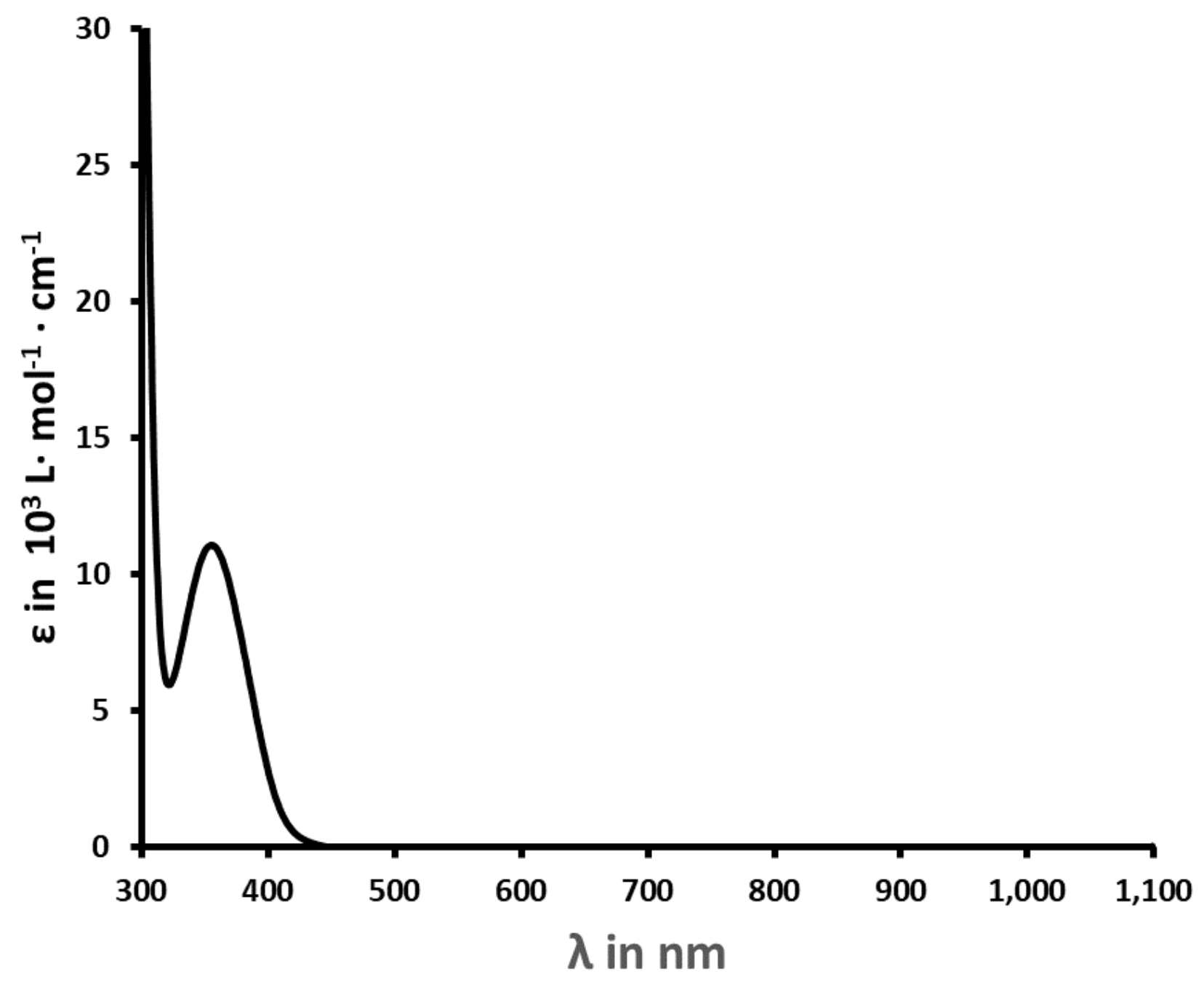

Figure $\mathrm{S} 64$ : UV-Vis of $\left[\mathrm{Co}\left(\mathrm{Cp}^{*}\right)_{2}\right][1]$ in $\mathrm{CH}_{2} \mathrm{Cl}_{2}$ at $298 \mathrm{~K}$. 


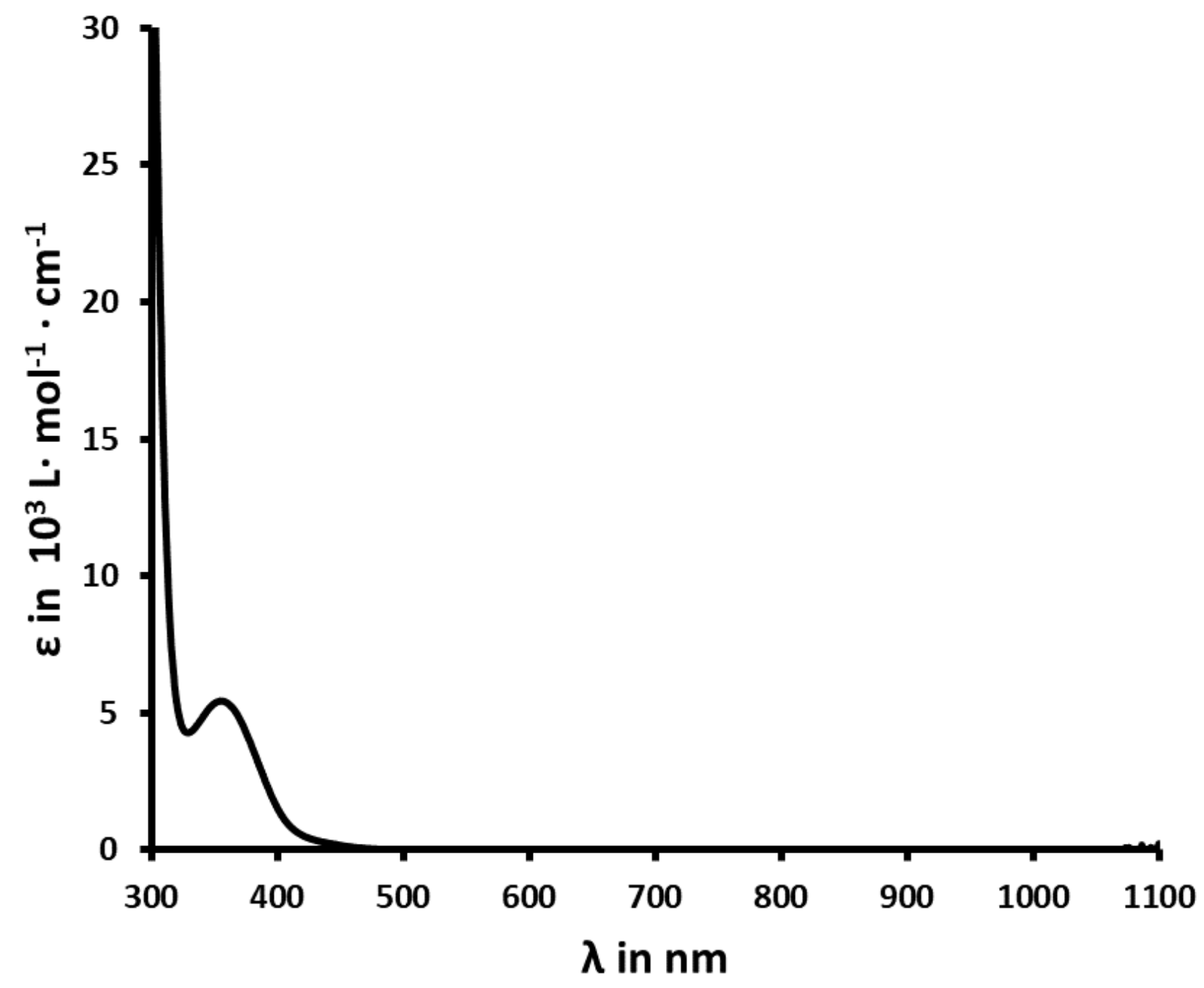

Figure $\mathrm{S} 65$ : UV-Vis of $\left[\mathrm{Co}\left(\mathrm{Cp}^{*}\right)_{2}\right][2]$ in $\mathrm{CH}_{2} \mathrm{Cl}_{2}$ at $298 \mathrm{~K}$. 


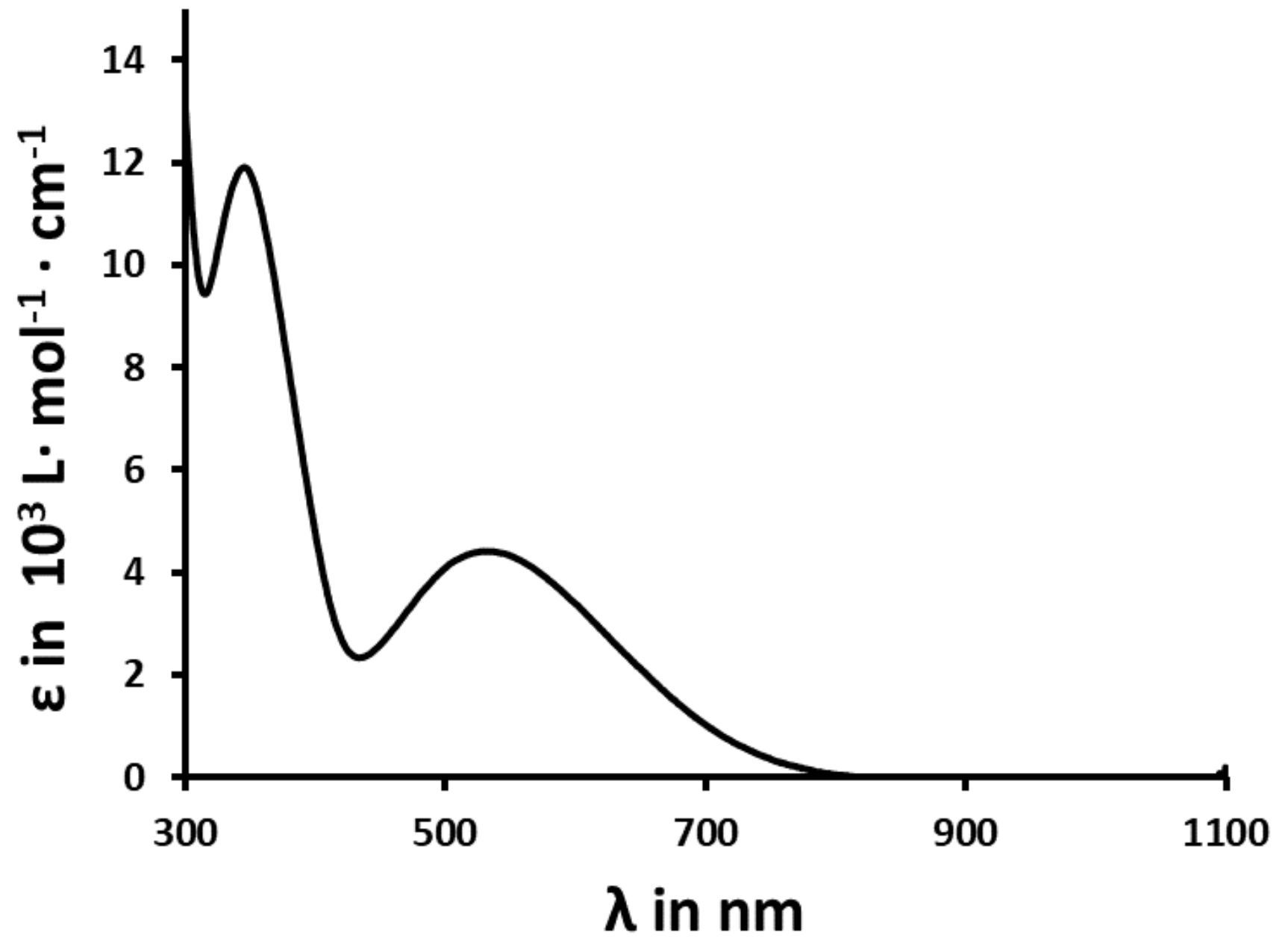

Figure S 66: UV-Vis of $\mathbf{4}$ in THF at 298K. 


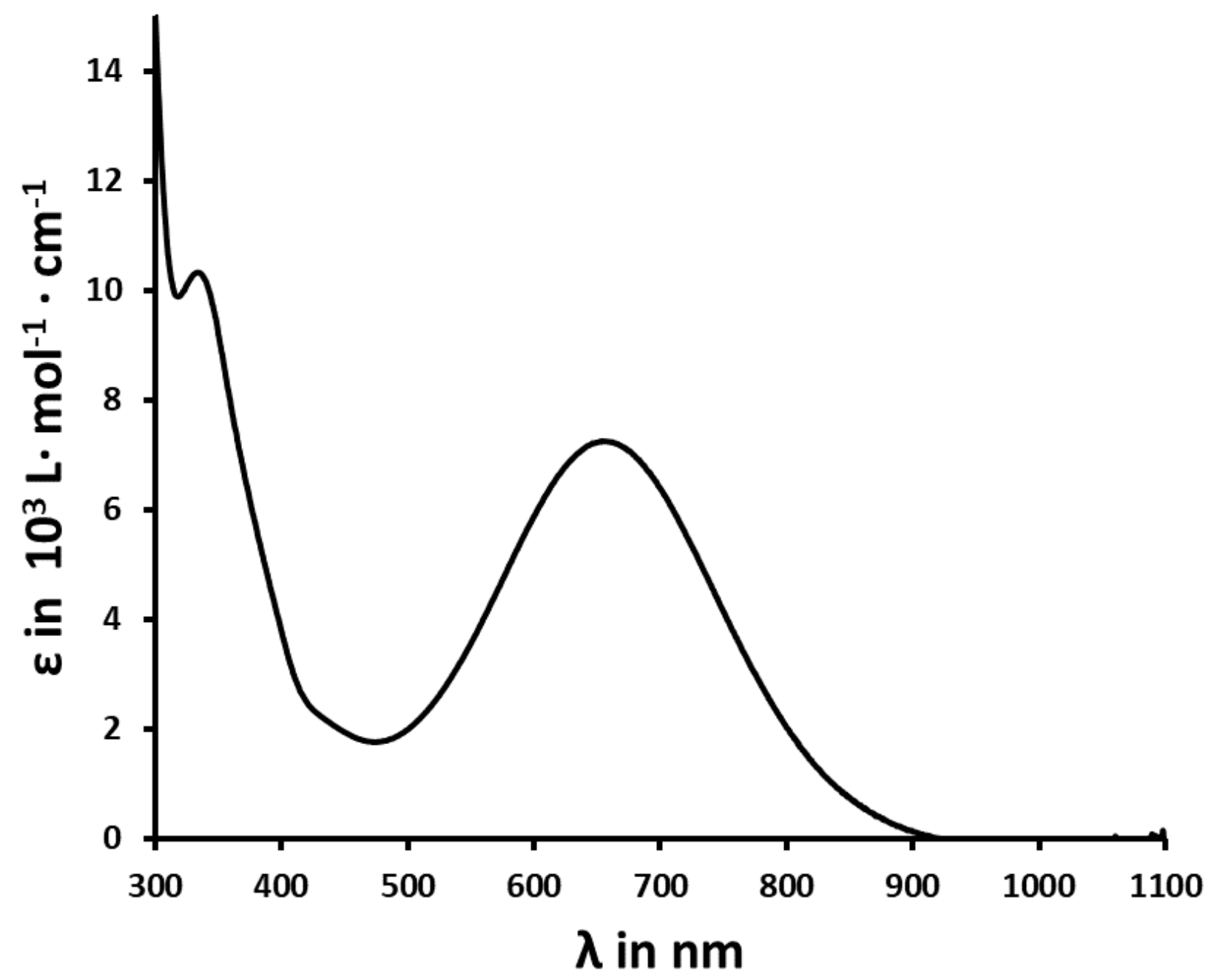

Figure S 67: UV-Vis of 5 in THF at 298K.

S 68 


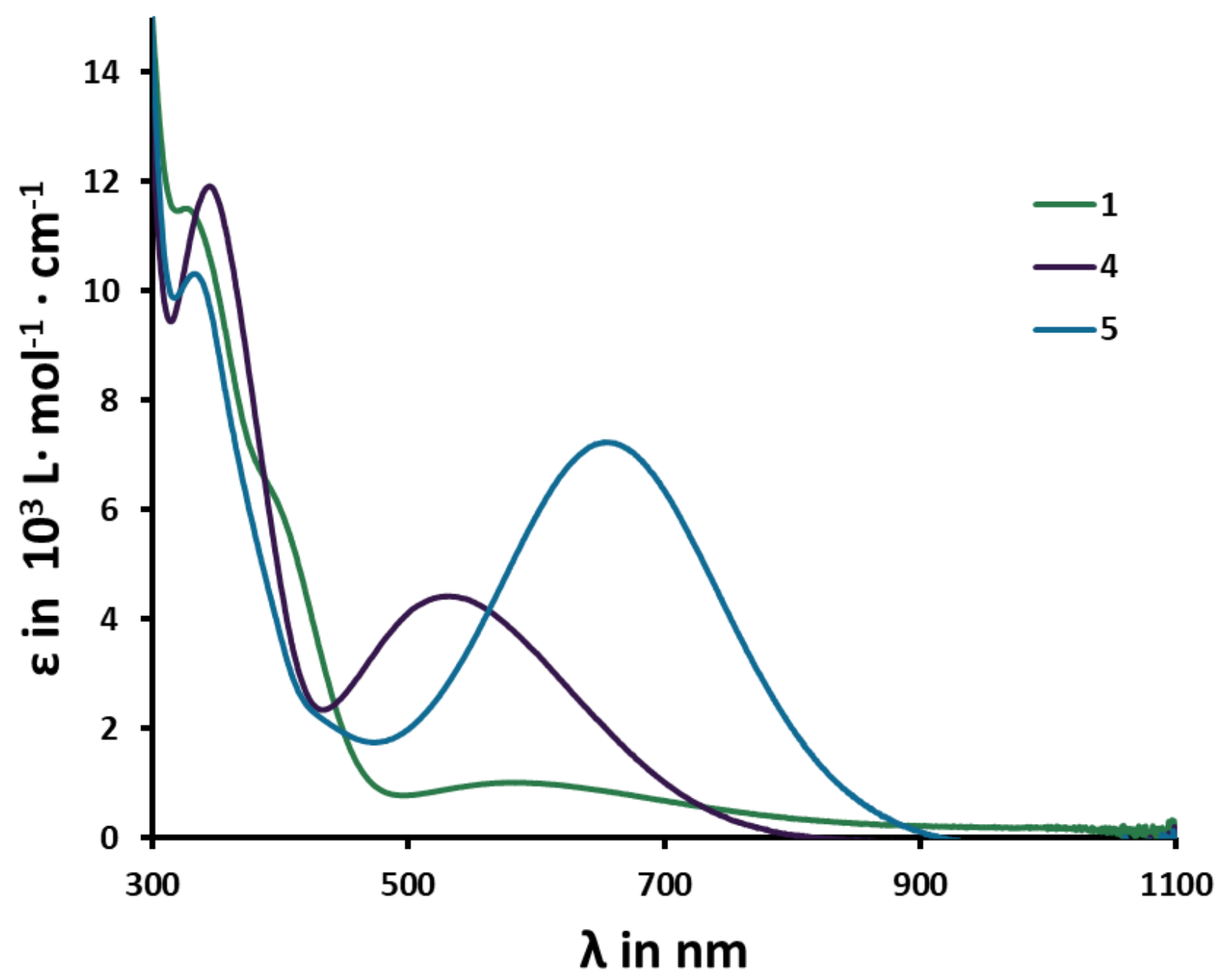

Figure S 68: Combined UV-Vis spectra for $\mathbf{1}, \mathbf{4}$ and $\mathbf{5}$ in THF at $298 \mathrm{~K}$. 


\section{Electrochemistry (Cyclic Voltammetry)}

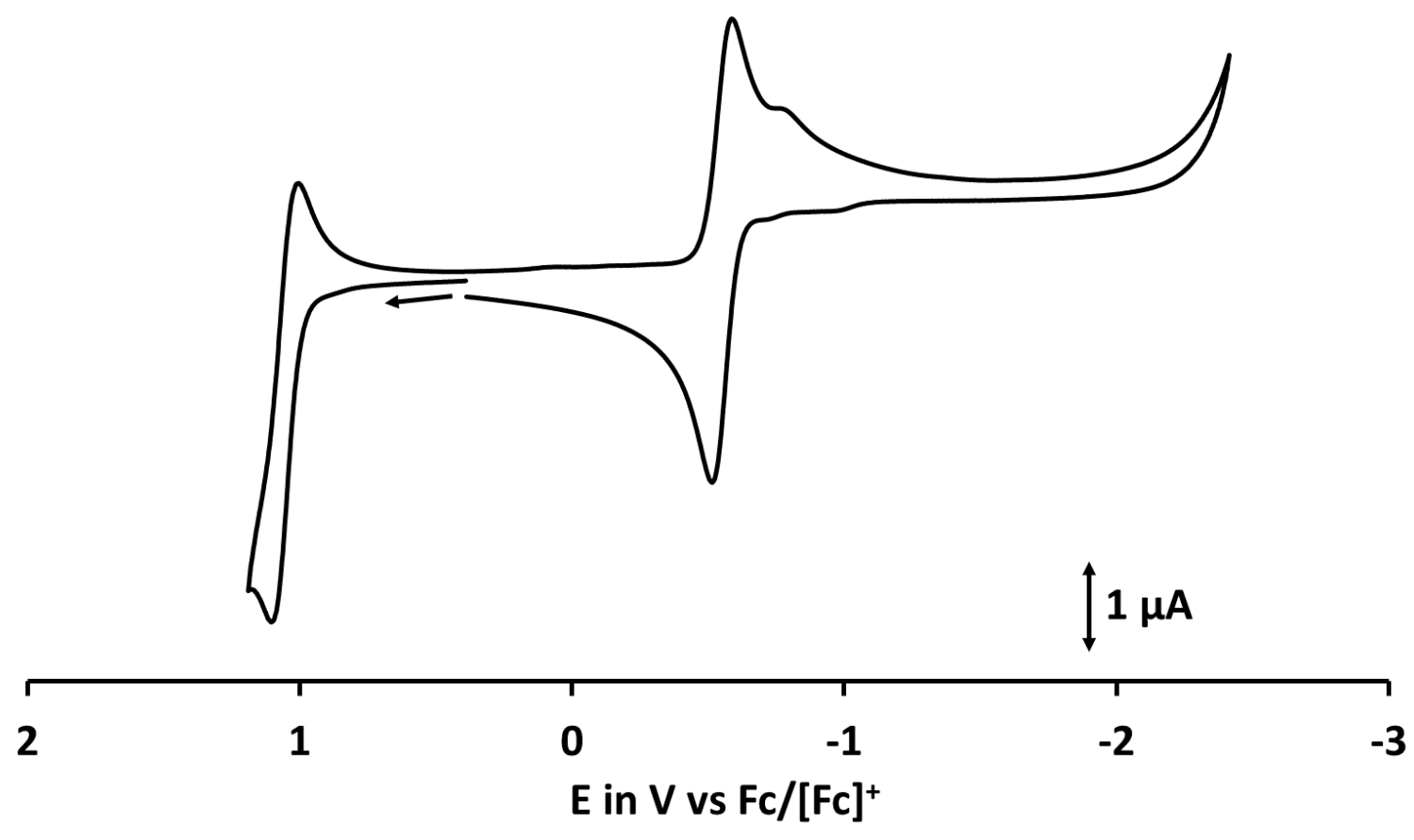

Figure S 69: Cyclic voltammogramm of 1 in $0.1 \mathrm{M} \mathrm{NBu}_{4} \mathrm{PF}_{6}$ in $\mathrm{CH}_{2} \mathrm{Cl}_{2}$ at $298 \mathrm{~K}$. Scan Rate $100 \mathrm{mVs}^{-1}$
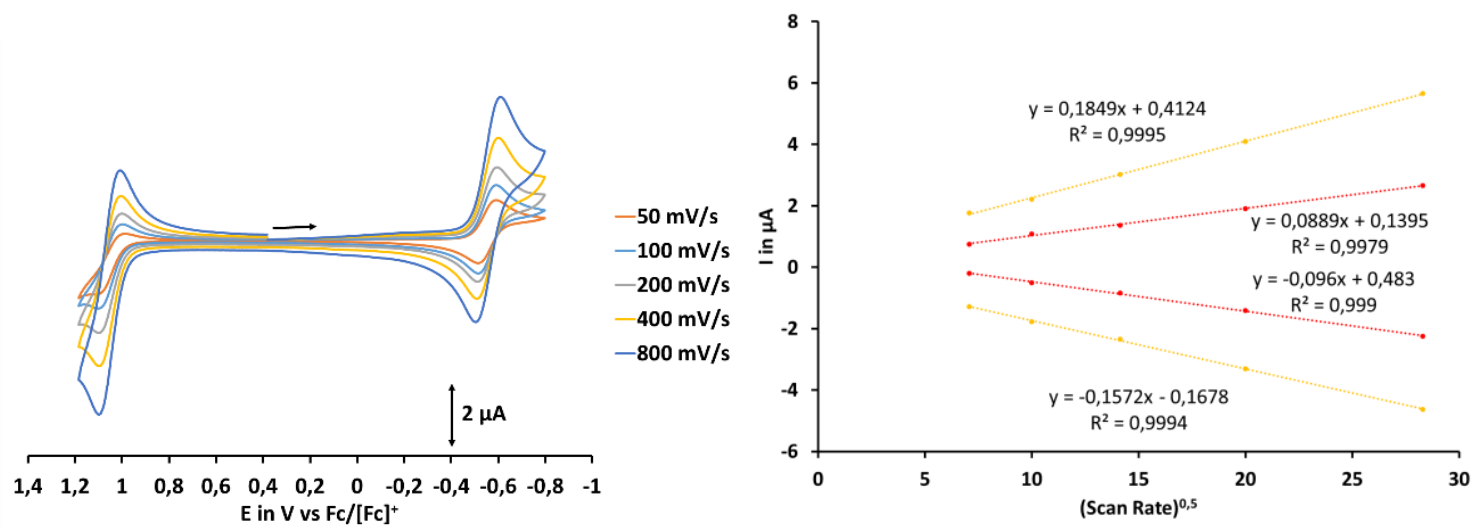

Figure S 70: Reversibility plots of the first reduction and the first oxidation of 1 Please note that the yellow lines in the left diagram belong to the first oxidation, while the red lines belong the first reduction process. 


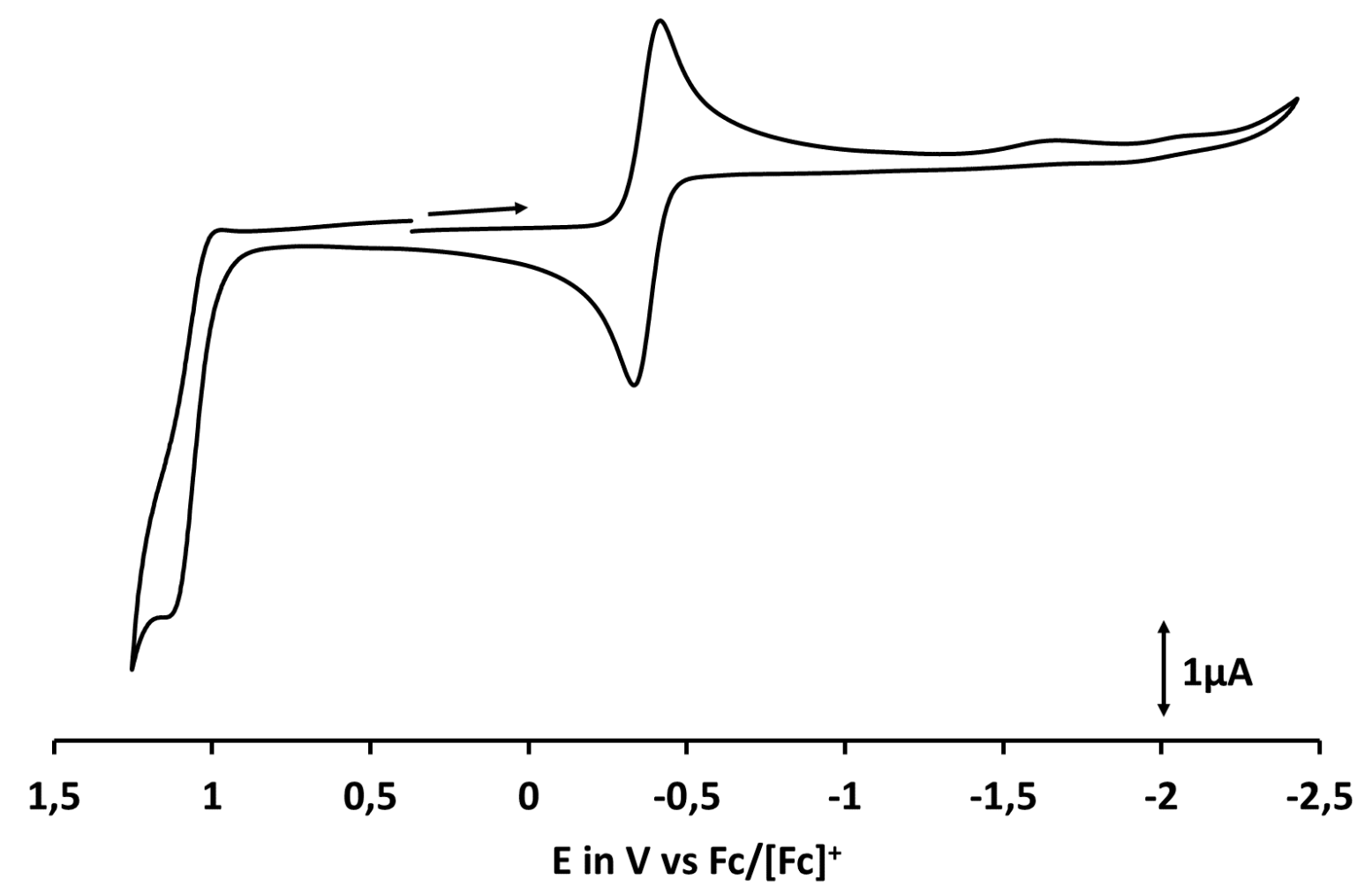

Figure S 71: Cyclic voltammogramm of 2 in $0.1 \mathrm{M} \mathrm{NBu}_{4} \mathrm{PF}_{6}$ in $\mathrm{CH}_{2} \mathrm{Cl}_{2}$ at 298K. Scan Rate $100 \mathrm{mVs}^{-1}$
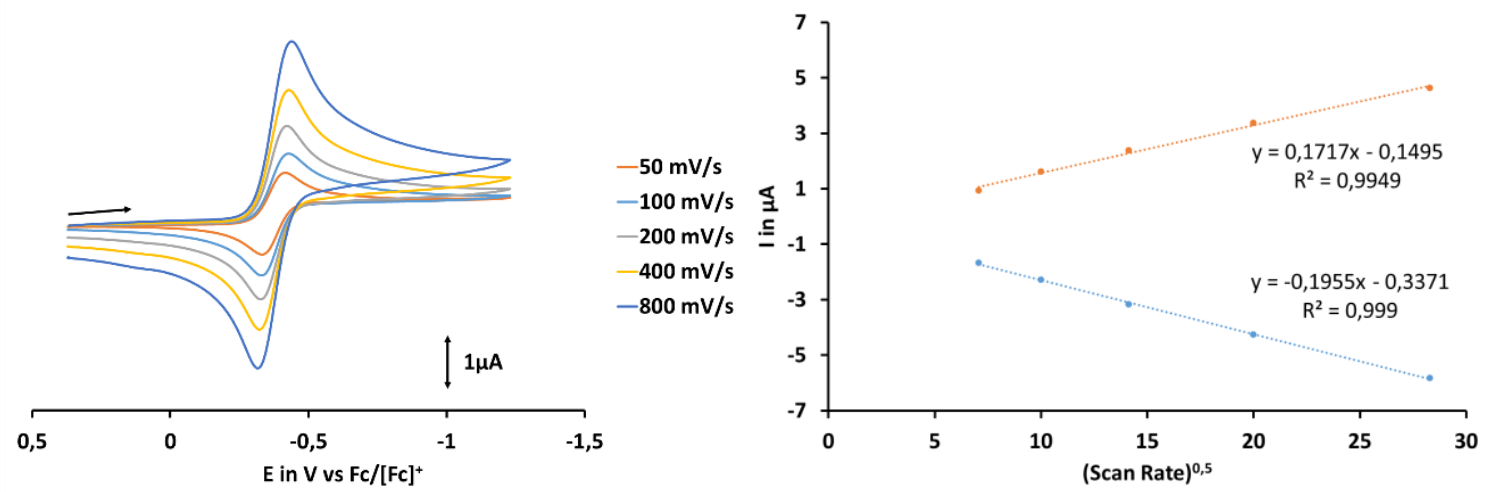

Figure S 72: Reversibility plots of the first reduction in $\mathbf{2}$. 


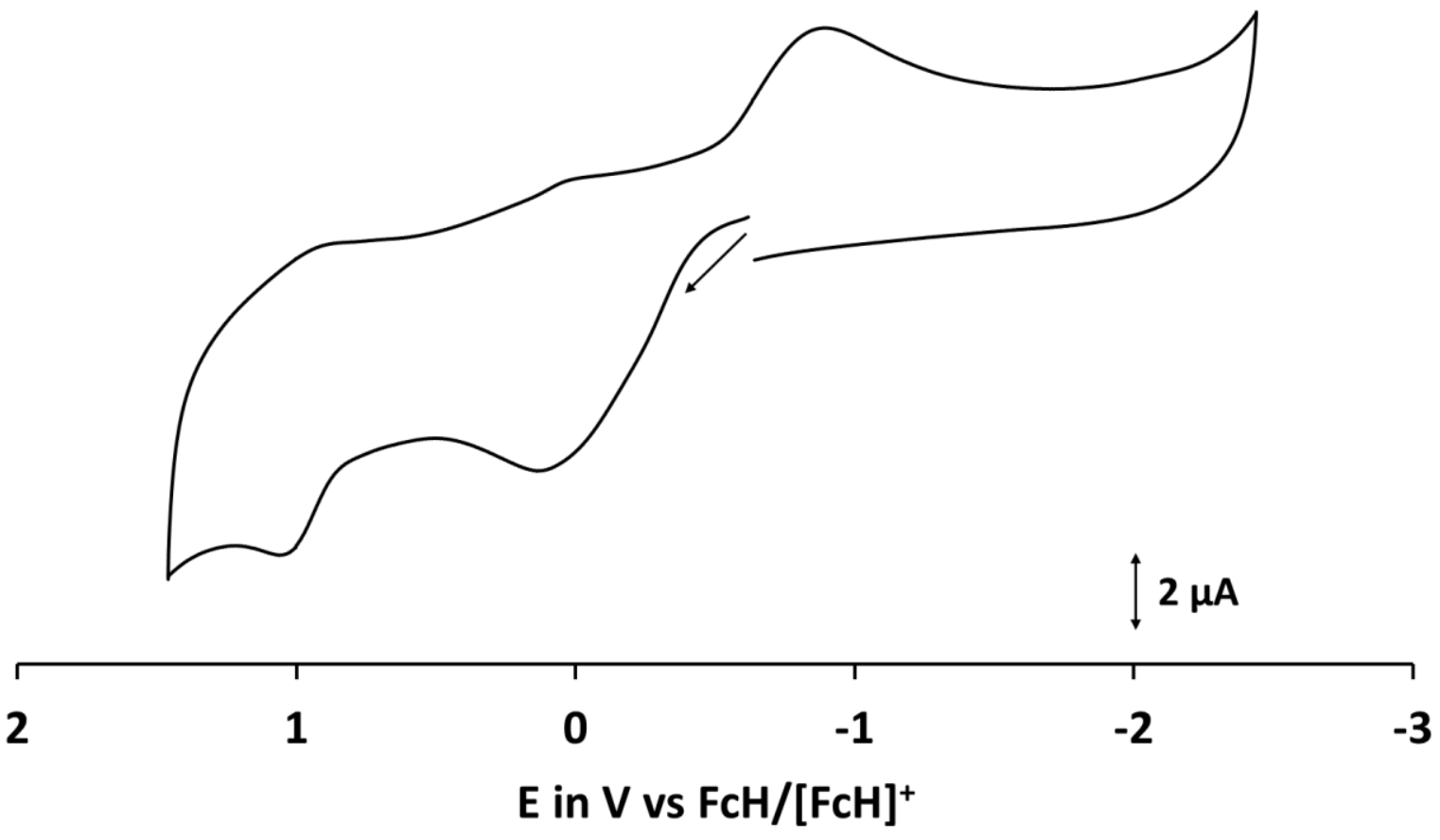

Figure S 73: Cyclic voltammogramm of 6 in $0.1 \mathrm{M} \mathrm{NBu}_{4} \mathrm{PF}_{6}$ in $\mathrm{MeCN}$ at 298K. Scan Rate $100 \mathrm{mVs}^{-1}$

\section{Quantum Chemical Calculations}

All calculations were performed with ORCA v. 4.2.1. ${ }^{1}$ The geometric parameters of $\mathbf{1}, \mathbf{2}$, as obtained from the solid-state structures, were optimized at the ZORA-PBE-D3BJ/ZORA-def2-SVP level of theory. ${ }^{2}$ For vanadium, the all-electron ZORA-def2-TZVP basis set was used. ${ }^{3}$ Scalar relativistic effects were modeled with the "Zeroth Order Regular Approximation" (ZORA). ${ }^{4}$ The RI approximation with the related auxilary basis set SARC/J was used to speed up the calculations. ${ }^{5}$ Tighter-than-default scf ("tightscf') and optimization criteria ("tightopt") were chosen in conjunction with finer-than-default grid values ("grid6"; "finalgrid7"). All calculated structures were verified as true minima by the absence $\left(N^{\text {imag }}=0\right)$ of negative eigenvalues in the harmonic vibrational frequency analysis. In case of compounds 2, two conformers were each obtained, whereas the calculations converged to only one minimum for compounds 1 . TD-DFT calculations were performed to model the experimental UV-Vis spectra. 50 roots were calculated at the ZORA-PBE/ZORA-def-TZVPP//ZORA-PBE-D3BJ/ZORA-def2-SVP as well as ZORA-PBEO/ZORA-def-TZVPP//ZORA-PBE-D3BJ/ZORA-def2-SVP level of theory. For the PBEO functional, the chain of spheres approximation (COSX) was used with finer than default grid value (gridx4). The TD-DFT calculations were performed using the Tamm-Dancoff approximation; the weight of the orbitals given below to illustrate the nature of the calculated absorption bands was found to be (typically considerably) larger than 0.75 . 
Table S 1: Energies of calculated compounds; values are given in [Eh].

\begin{tabular}{|c|c|c|c|c|}
\hline & $E(S V P)$ & G(SVP) & $E$ (TZVPP) & $E(P B E 0$, TZVPP) \\
\hline $1_{-}^{-} d$ & -3009.91345 & & -3011.74072 & 3011.87911 \\
\hline 1_s & -3009.84908 & -3009.23916 & -3011.67189 & -3011.78633 \\
\hline 1_t & -3009.78805 & & -3011.61206 & -3011.73874 \\
\hline 2_d & -3108.09705 & -3107.4607 & -3110.01755 & -3110.17175 \\
\hline 2-isomer_d & -3108.10613 & -3107.47197 & -3110.02848 & -3110.18464 \\
\hline 2_t & -3107.97446 & -3107.33828 & -3109.89223 & -3110.03536 \\
\hline 2_isomer_t & -3107.98012 & -3107.34394 & -3109.89886 & -3110.04327 \\
\hline 2_s & -3108.02928 & -3107.38985 & -3109.94584 & -3110.07388 \\
\hline 2_isomer_s & -3108.03519 & -3107.39575 & -3109.95238 & -3110.08265 \\
\hline 9_q & -3398.97129 & -3398.15215 & -3398.15215 & -3401.39744 \\
\hline 9_t & -3399.05277 & -3398.22861 & -3401.27253 & -3401.48935 \\
\hline 9_s & -3399.03494 & n.a. & -3401.25527 & -3401.4498 \\
\hline 9_S_UKS & -3399.0423 & n.a. & -3401.26217 & -3401.44772 \\
\hline
\end{tabular}

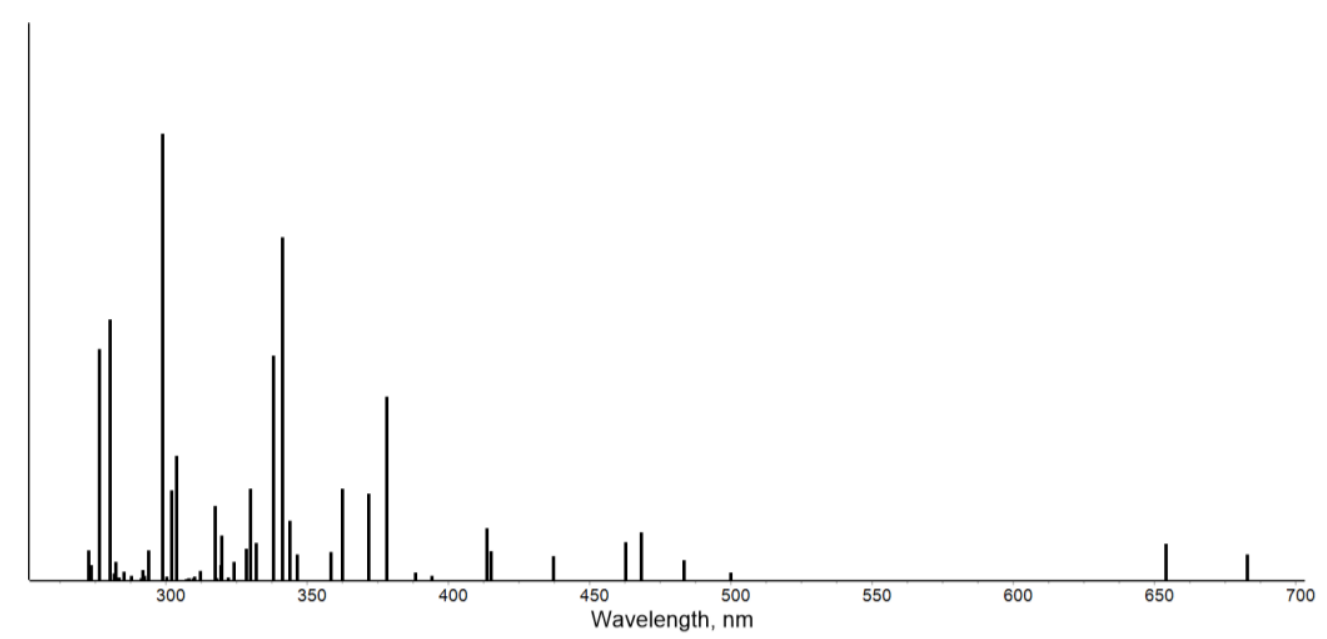

Figure S 74: Calculated (TD-DFT, PBE) transitions for 1.

LMCT (M0156 $\rightarrow$ MO157), 683 nm LMCT

LMCT (M0155 $\rightarrow$ M0157), 654 nm LMCT

LLCT (MO156 $\rightarrow$ MO158), 483 nm LLCT

LLCT (MO155 $\rightarrow$ MO158), 468 nm LLCT 


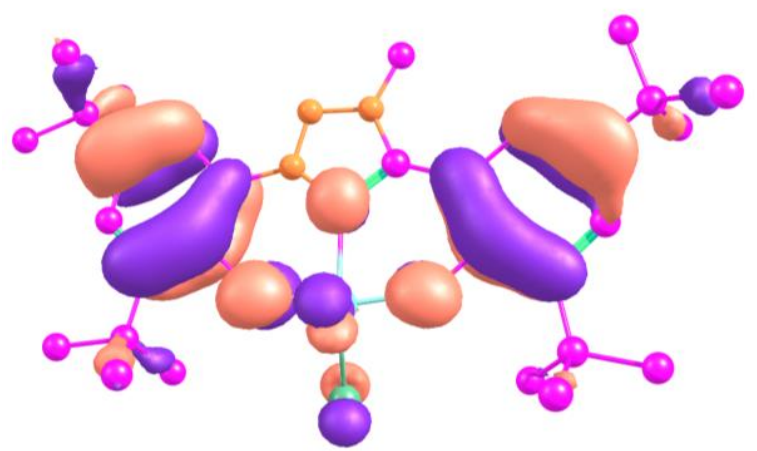

M0155

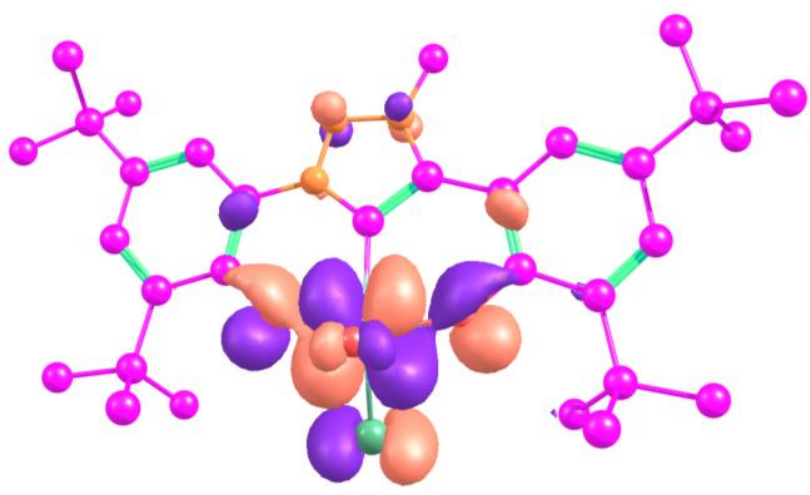

M0157

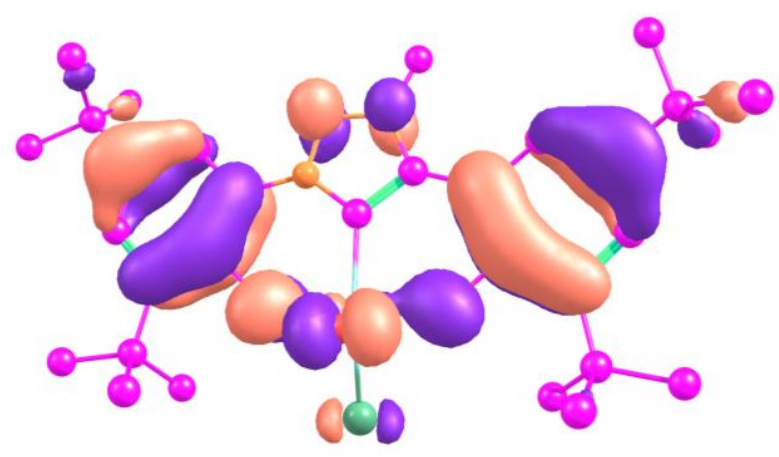

M0156

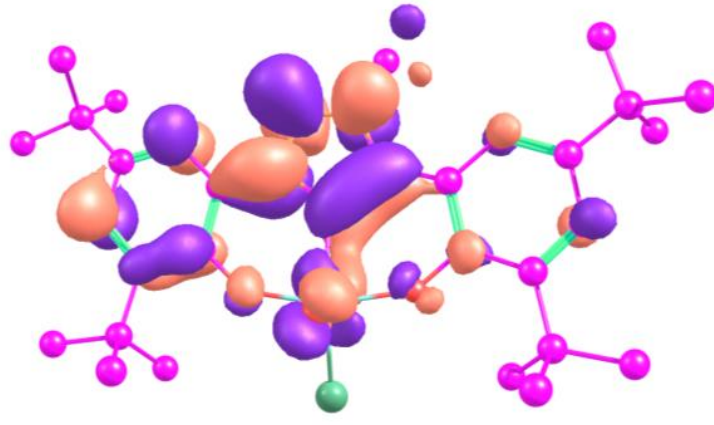

M0158

Figure S 75: Molecular orbitals associated with calculated (TD-DFT, PBE) transitions for 1.

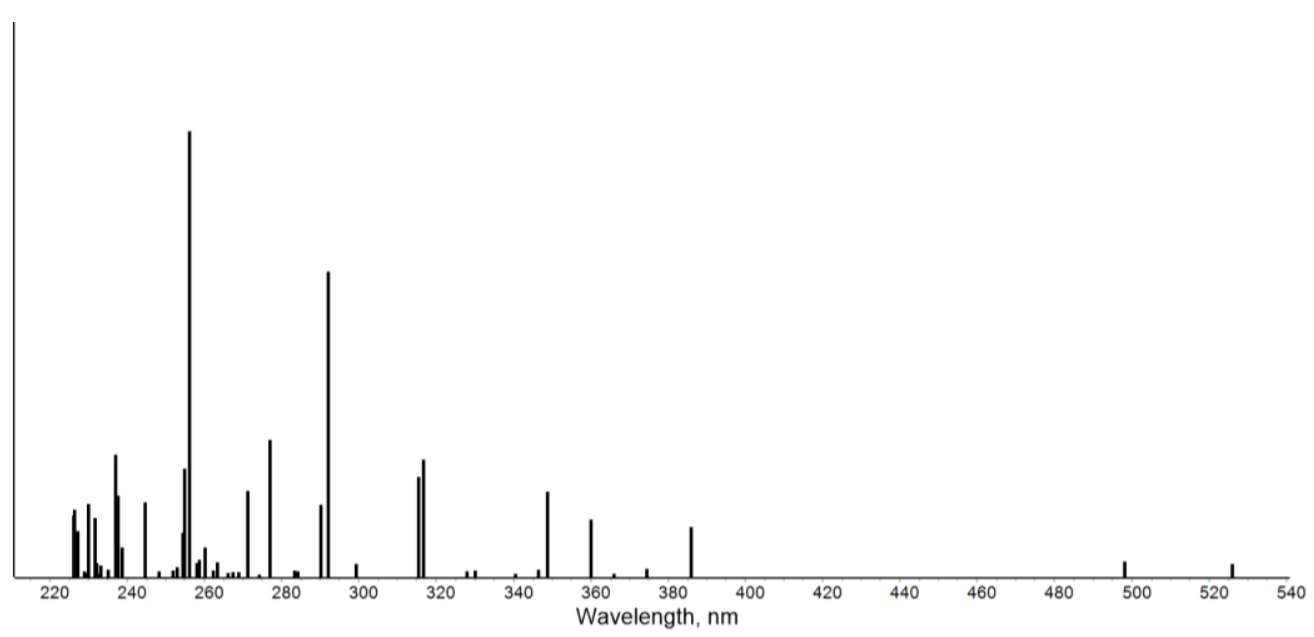

Figure S 76: Calculated (TD-DFT, PBE0) transitions for 1.

LMCT (M0156 $\rightarrow$ M0157), 526nm LMCT

LMCT (M0155 $\rightarrow$ MO157), 498 nm LMCT

LLCT (MO156 $\rightarrow$ MO158), 386 nm LMCT 


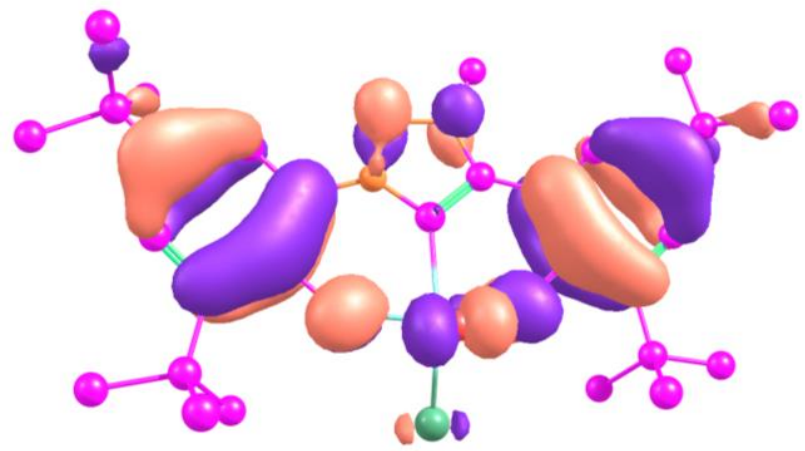

M0155

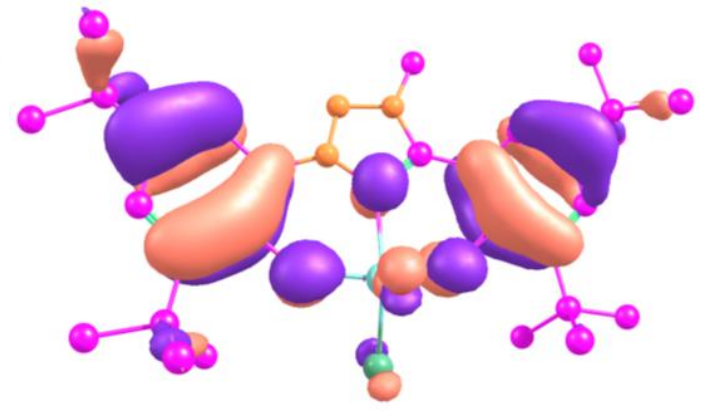

M0156

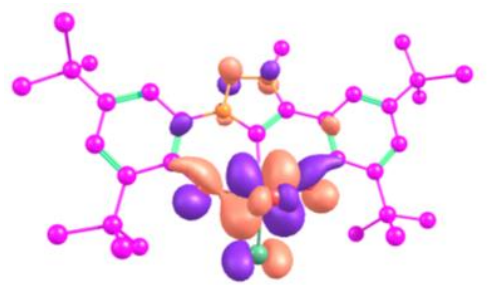

M0157

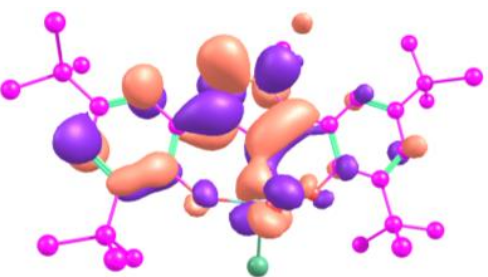

M0158

Figure S77: Molecular orbitals associated with calculated (TD-DFT, PBE0) transitions for 1.

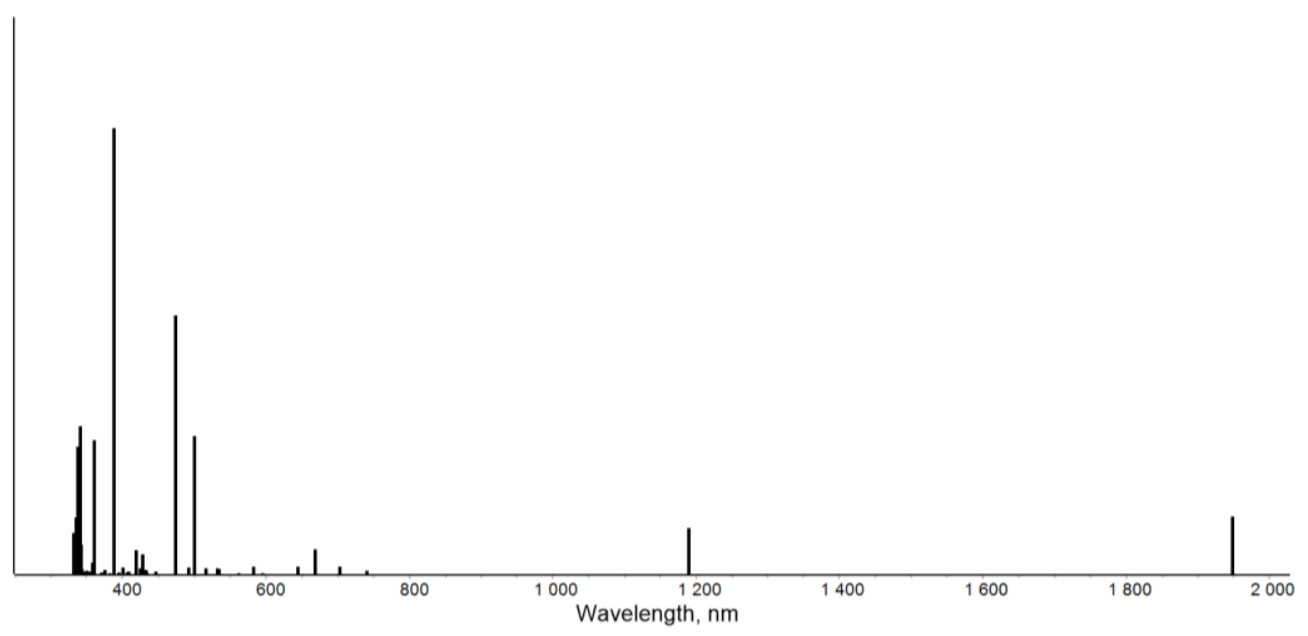

Figure S 78: $\quad$ Calculated (TD-DFT, PBE) transitions for 1-.

LLCT (M0155 $\rightarrow$ MO158), $500 \mathrm{~nm}$

LLCT (M0156 $\rightarrow$ MO158), $473 \mathrm{~nm}$ 


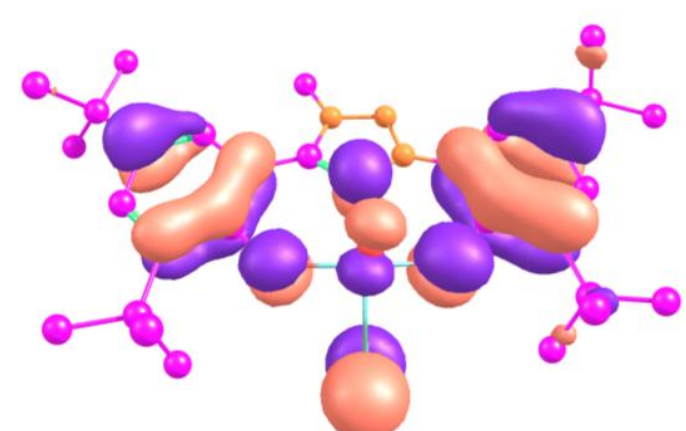

M0155

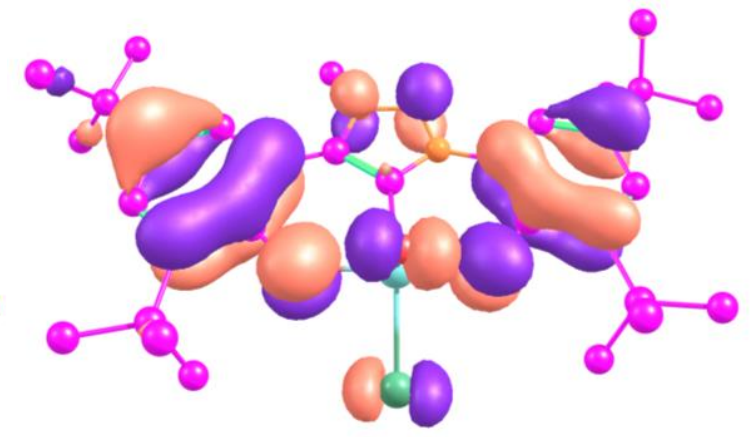

M0156

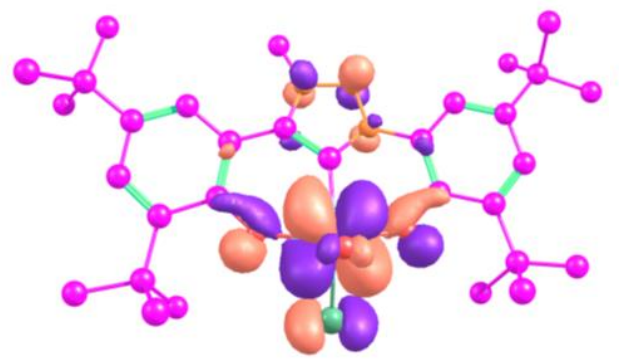

M0157 (SOMO)

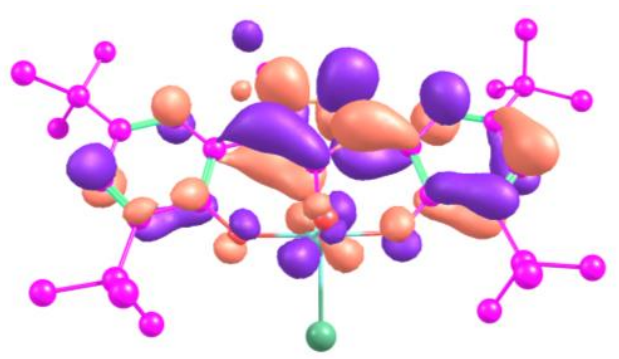

M0158

Figure S 79: Molecular orbitals associated with calculated (TD-DFT, PBE) transitions for 1.

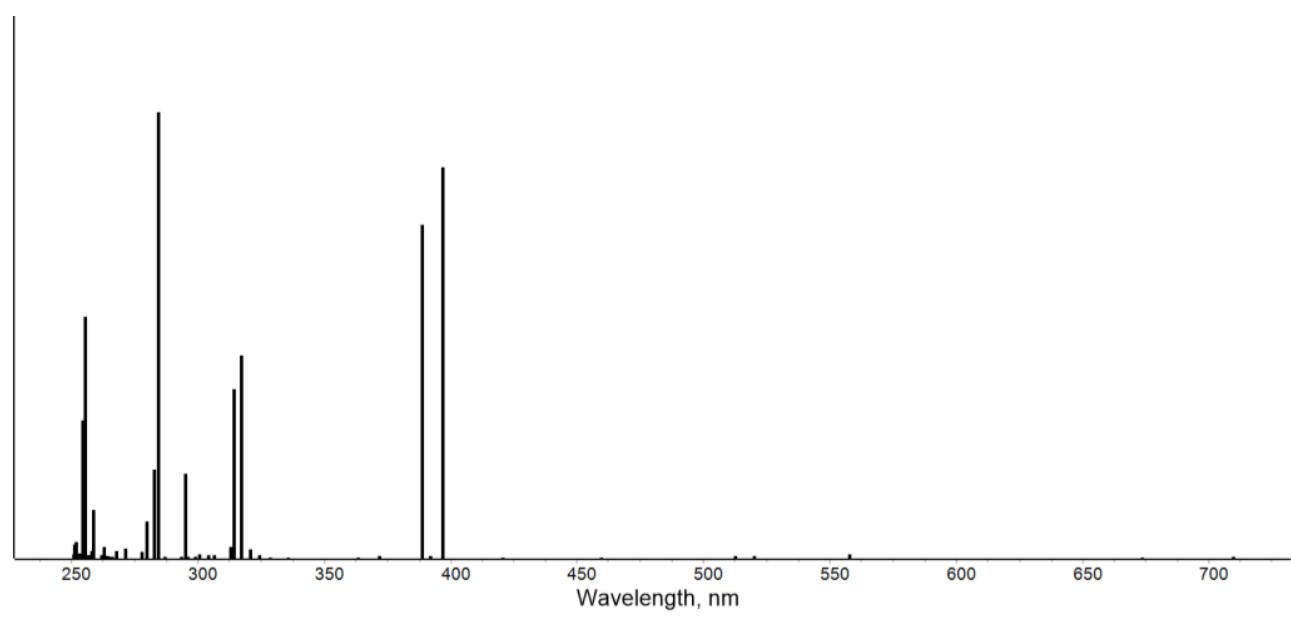

Figure S 80: Calculated (TD-DFT, PBE0) transitions for 1 :

LLCT (MO156 $\rightarrow$ M0158), $386 \mathrm{~nm}$

LLCT (MO155 $\rightarrow$ MO158), $357 \mathrm{~nm}$ 


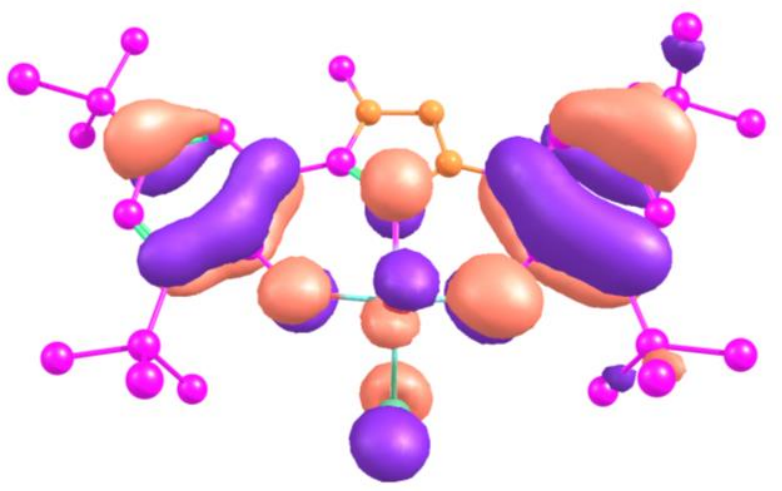

M0155

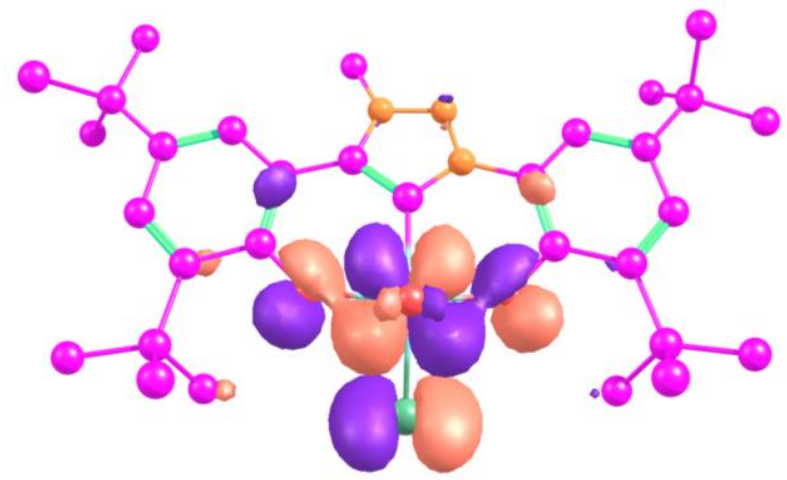

M0157 (SOMO)

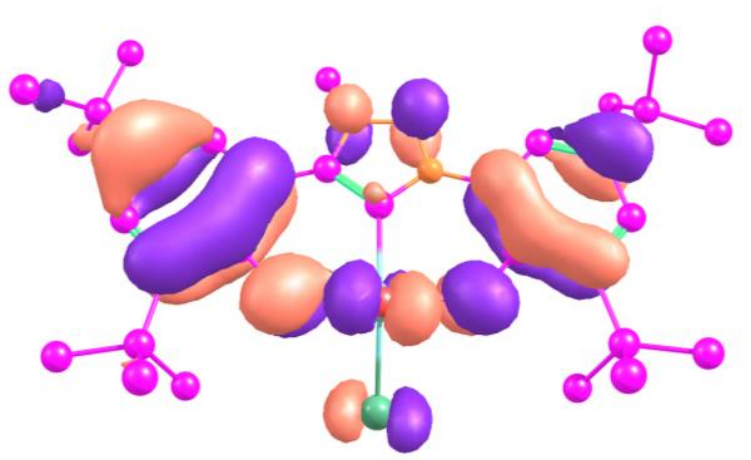

M0156

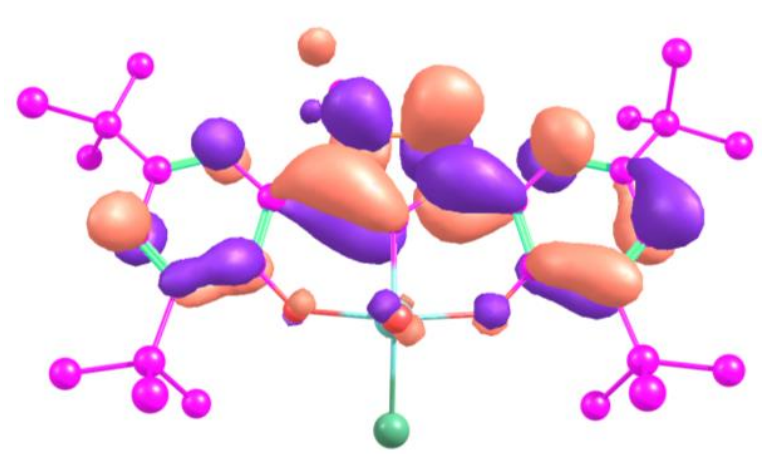

M0158

Figure S 81: Molecular orbitals associated with calculated (TD-DFT, PBE0) transitions for 1:

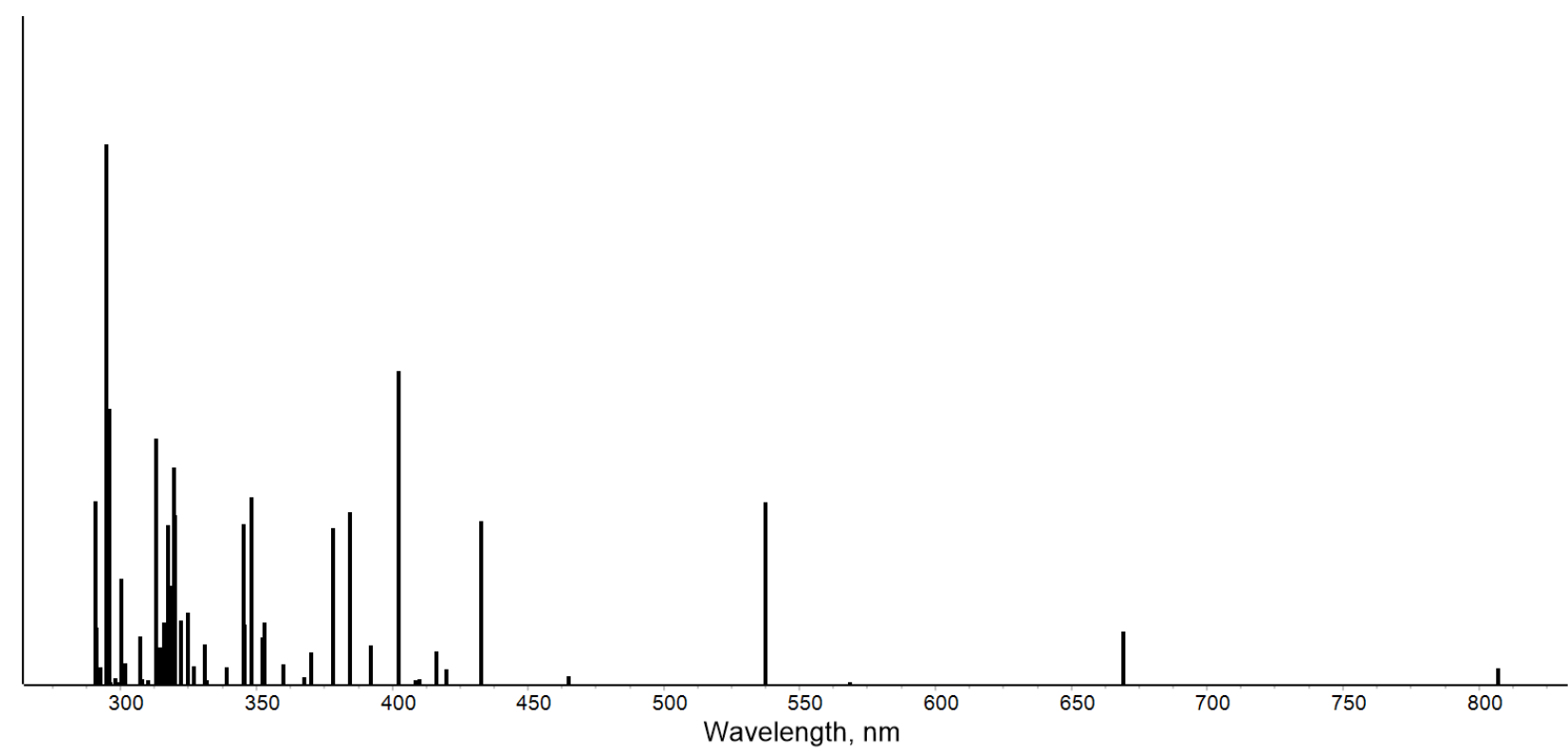

Figure S 82: $\quad$ Calculated (TD-DFT, PBE) transitions for 2. 


$$
\begin{aligned}
& \text { LMCT (M0166 } \rightarrow \text { MO167), } 807 \mathrm{~nm} \\
& \text { LMCT (M0165 } \rightarrow \text { M0167), } 669 \mathrm{~nm} \\
& \text { LLCT (MO166 } \rightarrow \text { M0168), } 537 \mathrm{~nm}
\end{aligned}
$$

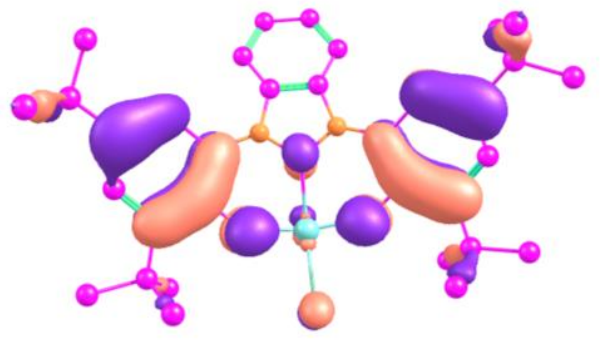

MO165

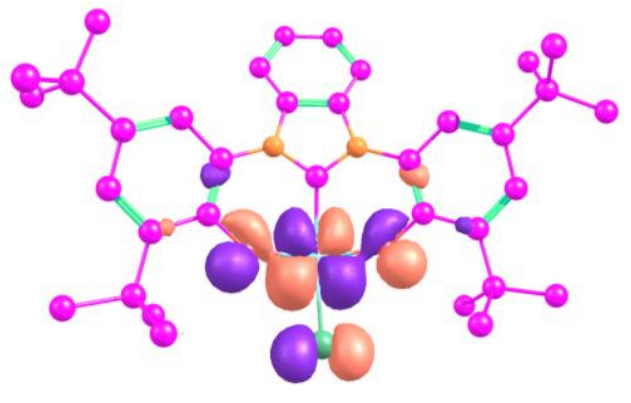

M0167

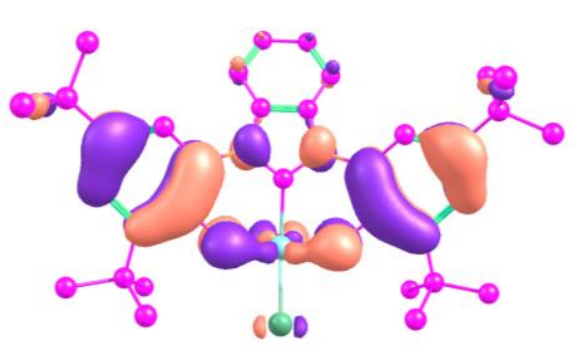

M0166

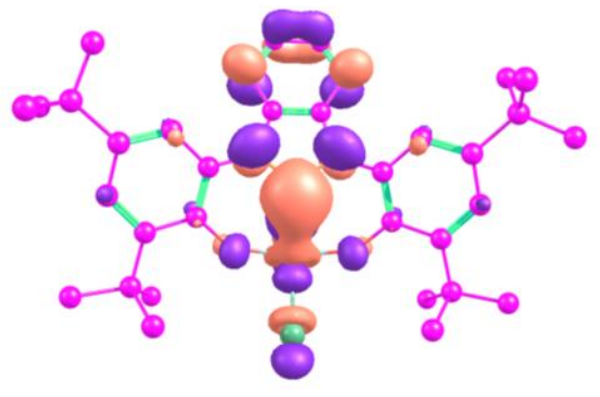

M0168

Figure S 83: Molecular orbitals associated with calculated (TD-DFT, PBE) transitions for 2.

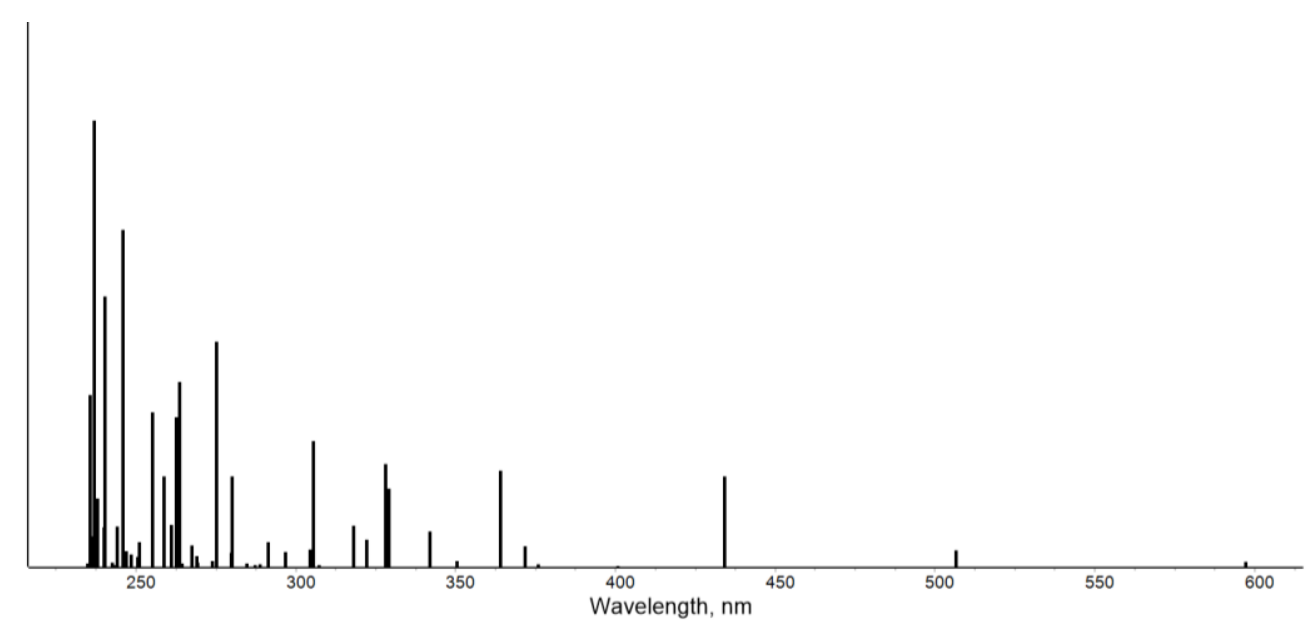

Figure S 84: Calculated (TD-DFT, PBE0) transitions for 2.

$$
\begin{aligned}
& \text { LMCT (M0166 } \rightarrow \text { M0167), } 597 \mathrm{~nm} \text { LMCT } \\
& \text { LMCT (M0165 } \rightarrow \text { M0167), } 506 \mathrm{~nm} \text { LMCT } \\
& \text { LLCT (M0166 } \rightarrow \text { M0168), } 434 \mathrm{~nm} \mathrm{LLCT}
\end{aligned}
$$




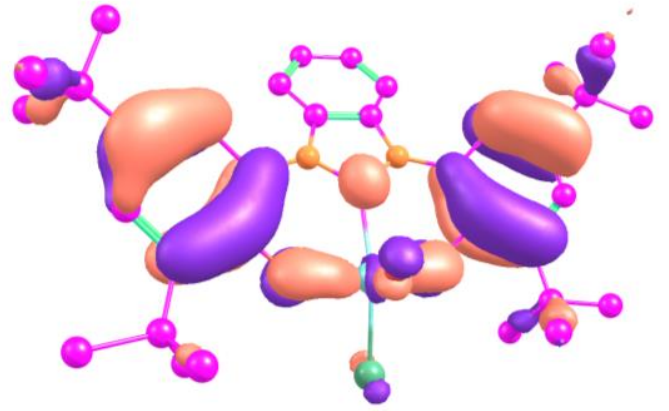

M0165

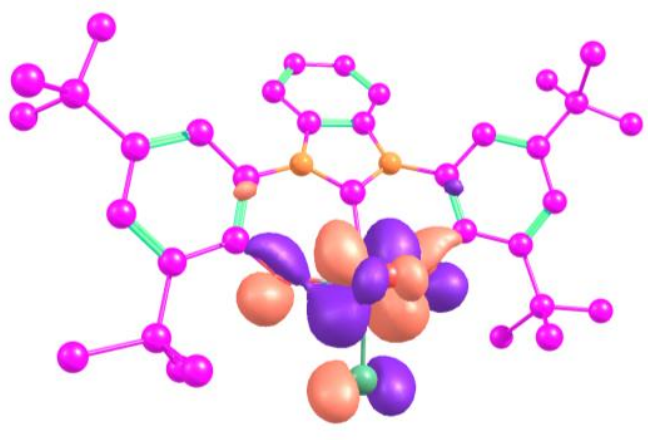

M0167

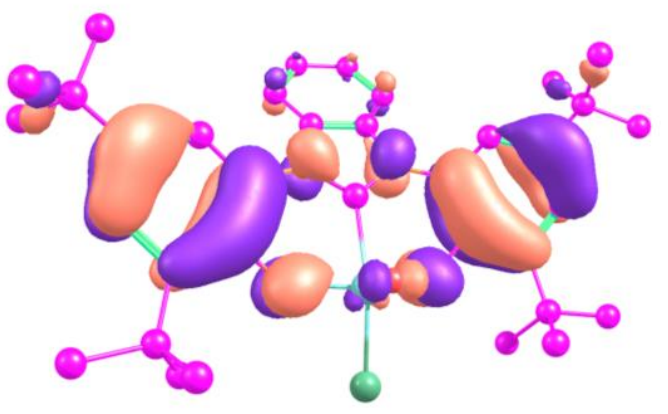

M0166

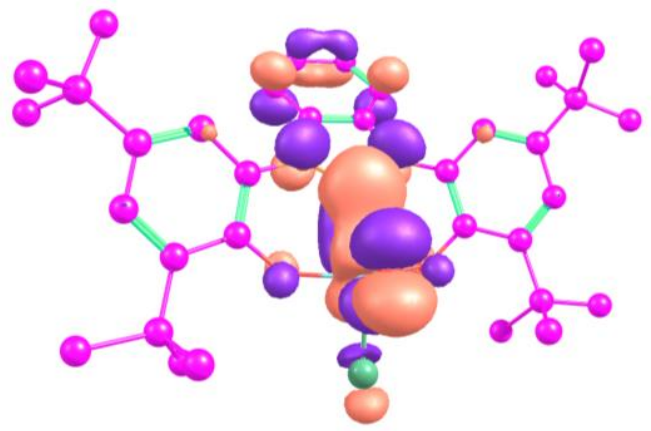

M0168

Figure S 85: $\quad$ Molecular orbitals associated with calculated (TD-DFT, PBE0) transitions for 2.

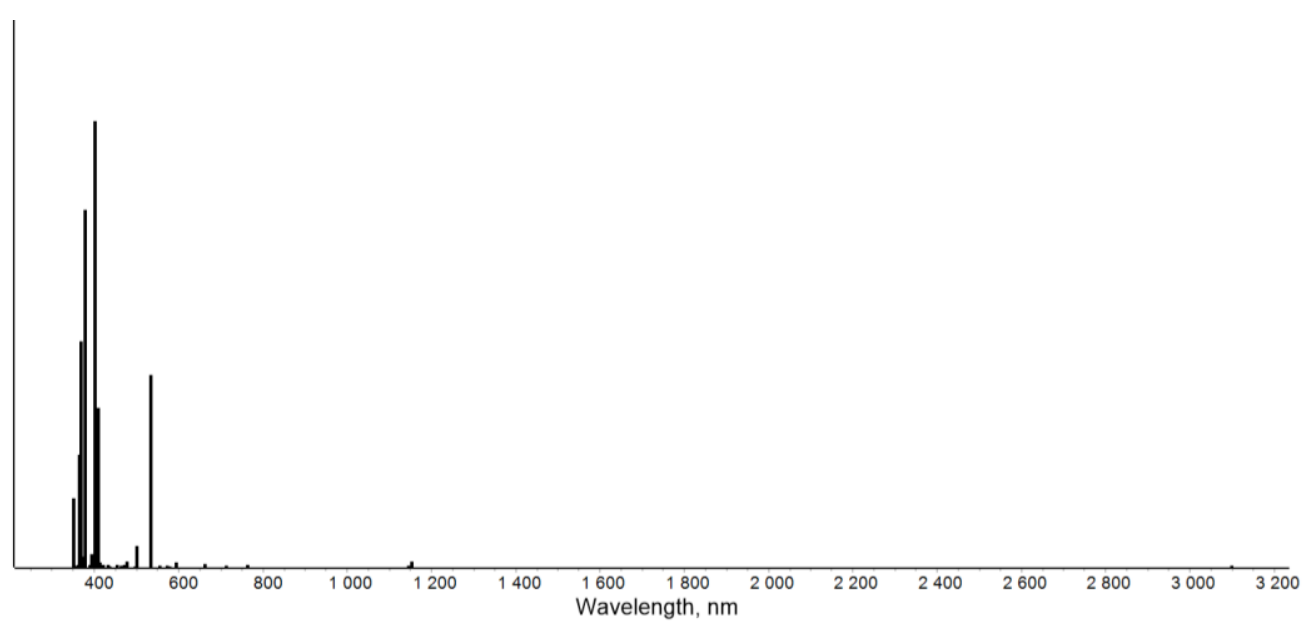

Figure S 86: $\quad$ Calculated (TD-DFT, PBE) transitions for $\mathbf{2}$.

LLCT (M0165 $\rightarrow$ M0167), 532 nm 


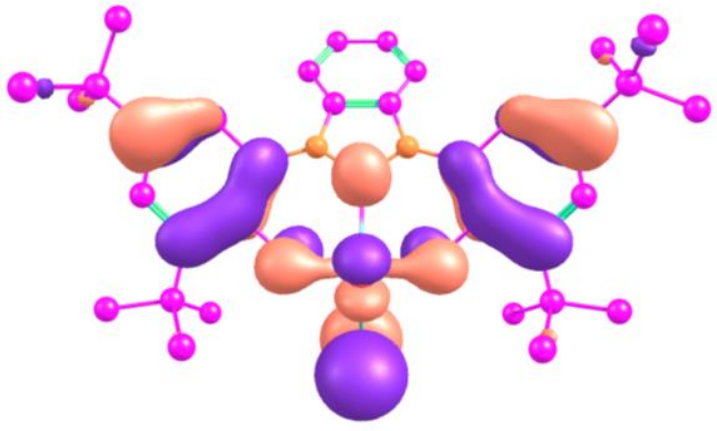

M0165

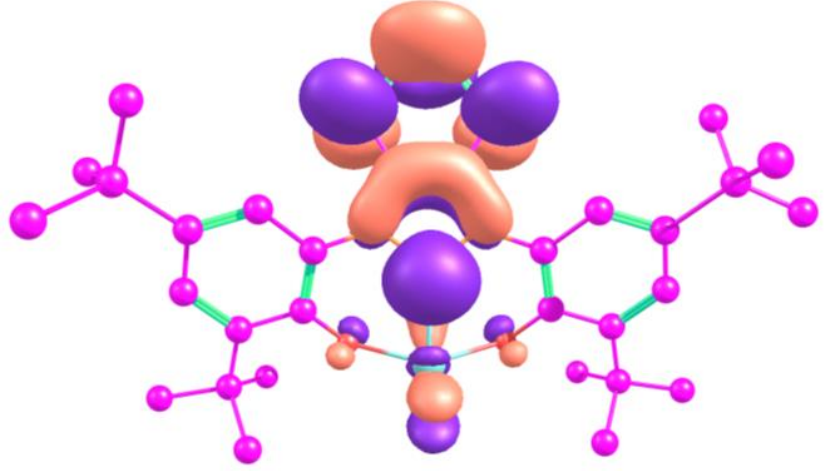

M0167

Figure S 87: Molecular orbitals associated with calculated (TD-DFT, PBE) transitions for 2:

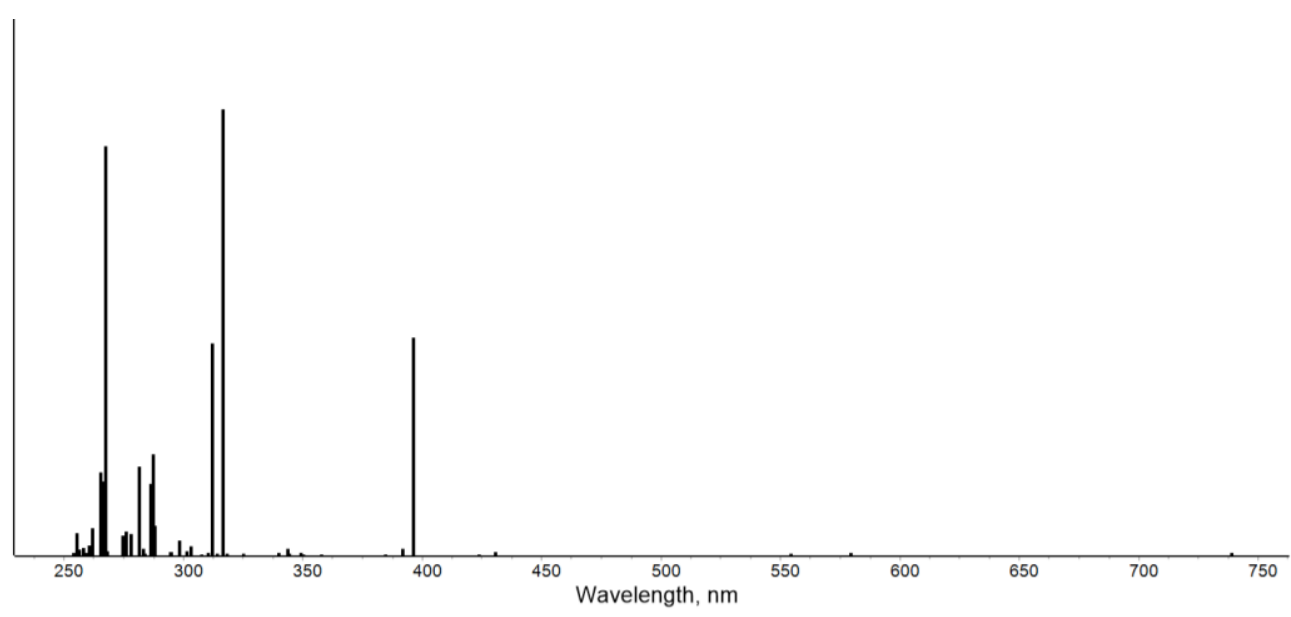

Figure S 88: Calculated (TD-DFT, PBE0) transitions for 2:

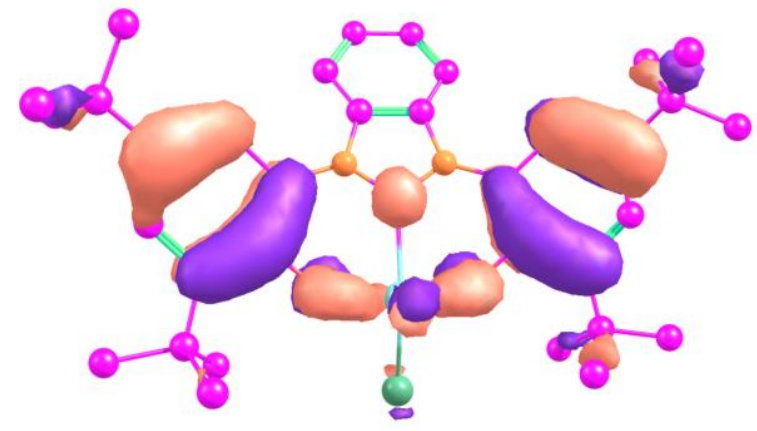

M0165

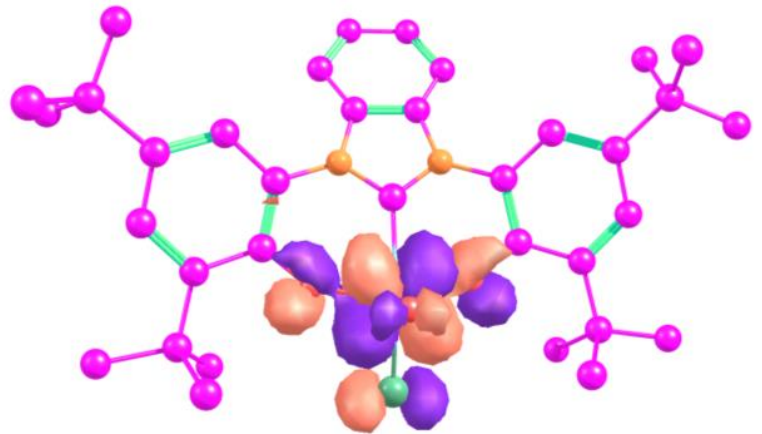

M0166 


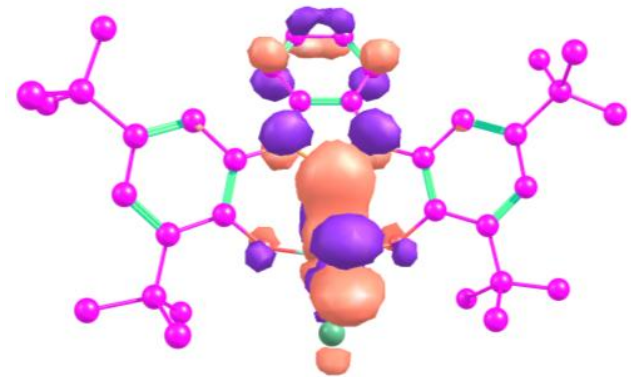

M0176

Figure S 89: $\quad$ Molecular orbitals associated with calculated (TD-DFT, PBE0) transitions for 2: 


\begin{tabular}{|c|c|c|c|}
\hline \multicolumn{4}{|c|}{${ }^{-}$-d } \\
\hline $\mathrm{V}$ & 3.36031568666981 & 15.41141833953448 & 12.11578300482809 \\
\hline $\mathrm{O}$ & 3.50131634989984 & 14.75983172769659 & 10.27949774355623 \\
\hline $\mathrm{N}$ & 5.99003923719238 & 13.91933919676474 & 11.31743988702488 \\
\hline $\mathrm{C}$ & 5.88469522685000 & 14.11346890057776 & 13.50019389355470 \\
\hline $\mathrm{C}$ & 5.19456603051946 & 14.44592386534269 & 12.31561165241519 \\
\hline $\mathrm{C}$ & 5.47987997429701 & 14.42118666773571 & 14.86142913763688 \\
\hline $\mathrm{C}$ & 4.12918190011037 & 14.90252425731538 & 15.03913425355397 \\
\hline $\mathrm{C}$ & 3.67084687065672 & 15.19195460505227 & 16.37884642178260 \\
\hline $\mathrm{C}$ & 4.45376660594690 & 14.40584637724942 & 9.46720162779355 \\
\hline $\mathrm{C}$ & 5.74117775810144 & 13.96462873168490 & 9.91940120364776 \\
\hline $\mathrm{C}$ & 6.33129318962552 & 14.28921291734943 & 15.98805722 \\
\hline $\mathrm{H}$ & 7.36796507878097 & 13.98524312495264 & 15.83831047140159 \\
\hline $\mathrm{C}$ & 4.23341445359935 & 14.42191412007491 & 8.04232892975002 \\
\hline $\mathrm{C}$ & 4.55971217307814 & 15.01189267145841 & 17.44118474216059 \\
\hline $\mathrm{H}$ & 4.20703090378915 & 15.23411600497886 & 18.45461949820884 \\
\hline $\mathrm{N}$ & 7.01988447858215 & 2344248854 & 13.08957170 \\
\hline $\mathrm{C}$ & 1.20743586699852 & 710833958696 & 88129208206 \\
\hline $\mathrm{H}$ & 1.30205025320645 & 5616559817 & 82593 \\
\hline $\mathrm{H}$ & 0.18101097056478 & 121517415 & 2013377016 \\
\hline $\mathrm{H}$ & 1.34188474440412 & 17225815 & 65390 \\
\hline $\mathrm{C}$ & 2.23542439582477 & 20507623 & 42105 \\
\hline $\mathrm{C}$ & 2.87873158517977 & 6691 & 97828 \\
\hline $\mathrm{N}$ & 7.09627825444031 & 2113 & 45057 \\
\hline $\mathrm{C}$ & 6.74289110519667 & 71266 & 47689 \\
\hline $\mathrm{H}$ & 7.69389914108935 & 68108 & 67161 \\
\hline $\mathrm{C}$ & 1.72647337776531 & 13249875 & 8.05524599064278 \\
\hline $\mathrm{H}$ & 1.88108483645370 & 653075860 & 68083880734 \\
\hline $\mathrm{H}$ & 0.76453769155663 & 70013292387 & 530197 \\
\hline $\mathrm{H}$ & 1.65152106114623 & 77030588887 & 591067 \\
\hline $\mathrm{C}$ & 5.89945751538395 & 14.57478632805354 & 17.28463634366801 \\
\hline $\mathrm{C}$ & 1.92604140770994 & 15.92205146055028 & 18.08994191544628 \\
\hline $\mathrm{H}$ & 2.00935900570238 & 14.98369072913324 & 18.66991402419105 \\
\hline $\mathrm{H}$ & 0.88902950212307 & 16.28916442348993 & 662621482544 \\
\hline $\mathrm{H}$ & 2.59358261142158 & 16.67440414406059 & 18.55027751071207 \\
\hline $\mathrm{C}$ & 6.52526492780425 & 13.54647126578226 & 7.64737663632358 \\
\hline $\mathrm{C}$ & 5.26228536746205 & 13.99364441892965 & 7.19207251535837 \\
\hline $\mathrm{H}$ & 5.07488158627850 & 14.01012465778314 & 6.11552917854342 \\
\hline $\mathrm{C}$ & 6.80685930419476 & 14.44431640464213 & 18.52016601000289 \\
\hline $\mathrm{C}$ & 2.66226460280803 & 16.39027262728386 & 7.92030614680284 \\
\hline $\mathrm{H}$ & 2.67282900333565 & 16.49977168713380 & 9.01618703433256 \\
\hline $\mathrm{H}$ & 1.68599838365600 & 16.75060446863723 & 7.54378492126080 \\
\hline $\mathrm{H}$ & 3.45418693067696 & 17.03419019650941 & 7.49488060268135 \\
\hline $\mathrm{C}$ & 2.06091762496735 & 17.06692778359017 & 15.87487012554292 \\
\hline $\mathrm{H}$ & 2.76127087459365 & 17.81666969227456 & 16.28751060243172 \\
\hline $\mathrm{H}$ & 1.02984242073763 & 17.44126101842542 & 16.02113060063991 \\
\hline $\mathrm{H}$ & 2.24439062829780 & 16.97330671523710 & 14.79317722182791 \\
\hline $\mathrm{C}$ & 8.22353452364801 & 13.96381479278450 & 18.15534989613310 \\
\hline $\mathrm{H}$ & 8.20396252044454 & 12.97026164668753 & 17.67117087009638 \\
\hline $\mathrm{H}$ & 8.84174825674334 & 13.87982930493593 & 19.06816041689356 \\
\hline $\mathrm{H}$ & 8.72924816743911 & 14.66985086225103 & 17.47175700768370 \\
\hline $\mathrm{C}$ & 6.19187434828129 & 13.42313115528661 & 19.50614391379289 \\
\hline $\mathrm{H}$ & 25029 & 13.72929391460590 & 19.82466584556901 \\
\hline $\mathrm{H}$ & 6.81844995012950 & 13.32224169189409 & 20.41346815173639 \\
\hline
\end{tabular}


H 6.10390213571824

C 8.89224248273452

H 8.65592942158753

H 9.71622916049561

H 9.25842201839082

C 7.64345373483344

C 7.22290510273390

H 6.34568221534044

H 8.05199161005285

H 6.97102387737606

C 2.82036000561694

H 3.59506990075455

H 1.83593671004415

H 2.93903149283883

C 8.01090059561022

H 8.34938626917840

H 8.82311266593676

H 7.13480843088100

C 6.93060855765150

H 7.37660545110681

H 7.56767901782616

H 5.94341652694329

O 3.31304691184045

Cl 1.09796836294486

O 3.62434686827106

C 8.08056096090182

H 8.76733474602299

H 7.65420493007046

H 8.64027808267981

1_S

V 3.19761222524894

O 3.64249657951492

N 6.09163315565675

C 5.99898363458586

C 5.20826612776049

C 5.56537204532675

C 4.25163489439058

C 3.74051686379365

C 4.53046836331288

C 5.81051943895671

C 6.33618825432506

H 7.31444810309191

C 4.23145739893886

C 4.56906576700919

H 4.18868828781028

N 7.26506226781627

C 1.28027607933376

H 1.42116534878421

H $\quad 0.26395999223331$

H 1.33702703320239

C 2.32927738560857

C 2.85122725200111

N 7.32700742663655

C 6.78144637864103

H 7.74458645896384
12.42911470474391

13.97115760475612

15.02652131326994

13.64841169097356

13.92809465324393

13.07975163077696

13.15899690524668

12.52073715780588

12.81496114445743

14.19280086191551

14.84915815001514

15.48072099058428

15.21734425005356

13.81563210160958

11.61153494896495

11.49976021675045

11.25424598671500

10.95264112622278

15.81838691481213

16.56348572351565

15.74694872085447

16.20259117940375

15.06892377175742

14.82619101724858

16.98313146190742

12.79632653390738

13.54193536989002

12.18015286773140

12.15763475979869

14.85617613980170

14.86055831446618

14.03818737621171

14.24776814055339

14.33844866450736

14.48387618697107

15.03143096126365

15.33365406240282

14.43444491733926

13.99144934469293

14.20173094156217

13.73366581596803

14.41659185750115

15.05079947585174

15.27313891652586

13.91275876581136

14.90537319010708

14.67114637944987

15.32127668184029

13.96233519075188

15.92199158361379

14.87448006187961

13.78337172504649

13.53495493059565

13.20147574756730
19.03174940118856

6.89215989017490

6.66645839959086

6.22656788827599

7.93277515642192

6.69713028066163

5.21798407011114

5.00870070541037

4.57219707740944

4.92047868963751

5.96156718984878

5.48802534086640

5.61938155423594

5.58571991052925

7.01617568397247

8.06111278721584

6.35371524916899

6.87827505953902

19.21989765802731

18.53693514894612

20.12269931745895

19.53056077263020

14.04212345387295

12.05868836700373

11.98274319512791

13.84699086519666

14.28713528110797

14.65559048932353

13.14756756415363

12.17146778913163

10.37304479033076

11.31358195979519

13.49599685203444

12.33015578992541

14.86107041663321

15.01204017044507

16.31494285168782

9.49412189404158

9.92098717650527

16.01617929023463

15.91038782870782

8.09647792190710

17.40649303398374

18.40833263098733

13.05243329925978

15.97230566920364

14.90593555229871

16.09808488429362

16.54537098491411

16.48836029154489

7.59444274679907

11.72942869600555

9.01797432145420

9.41673128419252 
C 1.75772520295882

H 1.91744392121642

H $\quad 0.76293398729157$

H 1.74531094262039

C 5.85974472700202

C 2.01530720425124

H 2.03505060250347

H 0.99984773140598

H 2.71706924654586

C 6.50766803965395

C 5.23288456290517

H 5.00521940216715

C 6.66800327485841

C 2.59056832478144

H 2.55663853703290

H 1.61844574251229

H 3.37490239391987

C 2.20842512662780

H 2.96722256305150

H 1.21060789006289

H 2.32580491554440

C 8.04531914874835

H 7.95478848622648

H 8.59354384039650

H 8.66612144261147

C 5.88165111546538

H 4.89512882231138

H 6.44048577335826

H 5.71199653204201

C 8.84402740961964

H 8.61560446095658

H 9.63065245485558

H 9.26059125189773

C 7.57933252812171

C 7.09907188065815

H 6.21033002896677

H 7.89834907671664

H $\quad 6.85111958425400$

C 2.75593302397687

H 3.49771551697623

H 1.75363932914950

H 2.89145683904345

C 7.93422168316340

H 8.32548383255662

H 8.70716918329761

H 7.04494505503602

C 6.88816616561924

H 7.45084003690013

H 7.45998077095091

H 5.93149529049566

O 3.50596374434936

O 2.42454053373906

Cl 1.83015383092360

C 8.51287238541271

H 8.68876181890109

H 8.48974134572465
13.94675282476745

12.90263083251727

14.26791709823633

13.96968283238137

14.47860227036640

16.21310969877617

15.29805205999387

16.63979613268806

16.94709068667017

13.51190230136699

13.95811501399600

13.94115209054653

14.17970649728054

16.33971441127897

16.45262578067564

16.67708633854151

17.00861285752830

17.25470831849214

17.97999127362812

17.69703182528522

17.11359532471705

13.56865079420003

12.60517316350598

13.37439388114975

14.25044428816249

13.17905740965140

13.57977366316588

12.95479381559256

12.23007525120204

13.88750537724226

14.94174520644815

13.53980701106571

13.85637992072167

13.00667823815877

13.05925589238021

12.42400799275180

12.69104581793250

14.08877713387269

14.80740909545503

15.46344812734892

15.14658298988339

13.77930997663739

11.54122689601584

11.44680880238369

11.15734869307909

10.89118914014920

15.49451178207620

16.22618082620298

15.30161809193508

15.96366918880794

15.26386093780447

13.47682221455567

16.63314276245236

13.73283531613011

14.59390660224361

12.80500754658324
8.18081713625482

7.85642551264403

7.82169397897938

9.28168444391693

17.29857105151130

17.96887507035504

18.58853373353109

18.04578211985884

18.40569972566757

7.64485581642244

7.22631896206160

6.15843778948142

18.57236767848603

8.02301310365044

9.11894613371052

7.61914452950774

7.62425681714928

15.70935481725581

16.05563586109765

15.88407177996087

14.62271927760100

18.25629702025969

17.72211665045245

19.19513552686396

17.64640962451798

19.45158523541186

19.74265838111307

20.37884993765858

18.91230105932266

6.79180681459678

6.55392061109769

6.09696944508832

7.81390316011258

6.66242349767393

5.20055651566893

5.03614243525554

4.53306255956366

4.88572227052533

6.05804649804548

5.56701107966561

5.74234574401103

5.67573160680827

7.00681689992478

8.03499743905417

6.31567695630701

6.92470648292572

19.35676652299043

18.74974967989851

20.28313048947600

19.64516521068344

13.94729949057897

12.29591689782847

11.90562908562499

13.77896396317681

14.44303407695393

14.37458330837621 


\begin{tabular}{|c|c|c|c|}
\hline \\
\hline 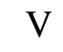 & 3.14788073191411 & 14.89857270963997 & 12.14980064139321 \\
\hline $\mathrm{O}$ & 3.56630948861174 & 14.94035333069374 & 10.23282172839999 \\
\hline $\mathrm{N}$ & 6.01226722243709 & 14.03984541917154 & 11.30677303685771 \\
\hline $\mathrm{C}$ & 5.93025495334569 & 14.26885680174630 & 13.48653396191971 \\
\hline $\mathrm{C}$ & 5.13530279770044 & 14.34348143074870 & 12.32390388020204 \\
\hline $\mathrm{C}$ & 5.51065683743106 & 14.51039909208535 & 14.85490638300768 \\
\hline $\mathrm{C}$ & 4.16594782825560 & 15.04205741200301 & 15.05334001290032 \\
\hline $\mathrm{C}$ & 3.69590790259874 & 15.30415589170021 & 16.40497487161985 \\
\hline $\mathrm{C}$ & 4.47003288669708 & 14.47734714798602 & 9.43663717622754 \\
\hline $\mathrm{C}$ & 5.74528050491358 & 13.99375561240355 & 9.91354072182160 \\
\hline $\mathrm{C}$ & 6.31205480545207 & 14.24544066869850 & 15.98624088730204 \\
\hline $\mathrm{H}$ & 7.30221477842254 & 13.81158540590284 & 15.85750743459478 \\
\hline $\mathrm{C}$ & 4.22666914350844 & 14.43936097842045 & 8.00828233014613 \\
\hline $\mathrm{C}$ & 4.56086157959328 & 15.02951103671544 & 17.45884174408912 \\
\hline $\mathrm{H}$ & 4.21528264992614 & 15.22671040072048 & 18.47810524275550 \\
\hline $\mathrm{N}$ & 7.20201179422116 & 13.91318720829274 & 13.04188914646855 \\
\hline $\mathrm{C}$ & 1.23341538592078 & 14.86241554690214 & 16.07856940040230 \\
\hline $\mathrm{H}$ & 1.34998924106491 & 14.69740076579700 & 14.99677958593898 \\
\hline $\mathrm{H}$ & 0.21795750169894 & 15.25939150782365 & 16.25908370310157 \\
\hline $\mathrm{H}$ & 1.31403431152207 & 13.88764946787956 & 16.59315302557092 \\
\hline $\mathrm{C}$ & 2.28264166877300 & 15.86185751532228 & 16.63107638374424 \\
\hline $\mathrm{C}$ & 2.88049097720513 & 14.93003394961082 & 7.45482611429152 \\
\hline $\mathrm{N}$ & 7.25872896423811 & 13.77132165741991 & 11.72260067261949 \\
\hline $\mathrm{C}$ & 6.72268837550534 & 13.51283776393607 & 9.03738528362820 \\
\hline $\mathrm{H}$ & 7.67064553220788 & 13.16565376027800 & 9.45937273506023 \\
\hline $\mathrm{C}$ & 1.73403402875051 & 14.06602734336319 & 8.04121318750346 \\
\hline $\mathrm{H}$ & 1.86219651593105 & 13.00491090675042 & 7.76031754898617 \\
\hline $\mathrm{H}$ & 0.76668828705965 & 14.41173739803434 & 7.63354380300134 \\
\hline $\mathrm{H}$ & 1.68812516425232 & 14.13394407281045 & 9.13896819303012 \\
\hline $\mathrm{C}$ & 5.86755829507372 & 14.49529607509828 & 17.29054033356573 \\
\hline $\mathrm{C}$ & 1.99048943719926 & 16.06721582363130 & 18.13043402030579 \\
\hline $\mathrm{H}$ & 2.04257538691152 & 15.12130517839162 & 18.70034278421269 \\
\hline $\mathrm{H}$ & 0.96734455765776 & 16.46521433808367 & 18.24674549569056 \\
\hline $\mathrm{H}$ & 2.68150915163566 & 16.79515164810602 & 18.59402955493974 \\
\hline $\mathrm{C}$ & 6.48967522069073 & 13.47899132323945 & 7.65203350337738 \\
\hline $\mathrm{C}$ & 5.23802226285289 & 13.94752535525919 & 7.17946665519632 \\
\hline $\mathrm{H}$ & 5.05084649266873 & 13.92144595564494 & 6.10415926596784 \\
\hline $\mathrm{C}$ & 6.72915897765707 & 14.20842723809244 & 18.52737748272583 \\
\hline $\mathrm{C}$ & 2.66715485338213 & 16.42078256149386 & 7.82479793268420 \\
\hline $\mathrm{H}$ & 2.62084241434307 & 16.57622202668705 & 8.91422029217340 \\
\hline $\mathrm{H}$ & 1.71575619626656 & 16.77394709531243 & 7.38665327502173 \\
\hline $\mathrm{H}$ & 3.48143281410582 & 17.04420434901732 & 7.41231591132714 \\
\hline $\mathrm{C}$ & 2.13621652985503 & 17.23540751723693 & 15.92727425286550 \\
\hline $\mathrm{H}$ & 2.88105560497626 & 17.95314731407704 & 16.31716967867992 \\
\hline $\mathrm{H}$ & 1.13100358720605 & 17.64619042851364 & 16.13372132010683 \\
\hline $\mathrm{H}$ & 2.25493411678046 & 17.16051215863445 & 14.83473198641294 \\
\hline $\mathrm{C}$ & 8.11102094111433 & 13.63694991531550 & 18.16099689489340 \\
\hline $\mathrm{H}$ & 8.02920242324728 & 12.67501842360601 & 17.62279587997956 \\
\hline $\mathrm{H}$ & 8.69354892921292 & 13.45066996678327 & 19.08036267633242 \\
\hline $\mathrm{H}$ & 8.69431354896693 & 14.33968623767183 & 17.53822688062514 \\
\hline $\mathrm{C}$ & 5.99852279186241 & 13.18120084983743 & 19.42597093549190 \\
\hline $\mathrm{H}$ & 5.01333146666760 & 13.55293289984683 & 19.75629616604455 \\
\hline $\mathrm{H}$ & 6.59818952496081 & 12.96812209205653 & 20.32979705132805 \\
\hline
\end{tabular}


H 5.83617673016482

C 8.85653242749518

H 8.65447493431715

H 9.65922395993361

H 9.23747860558548

C 7.58242055584555

C 7.15507440996837

H 6.26173079341689

H 7.97110177962521

H 6.93756236404167

C 2.82267209162241

H 3.59630146243448

H 1.84026128674184

H 2.93375613863604

C 7.90153147439052

H 8.25746365144176

H 8.69109448783056

H 7.00595136867796

C 6.93885494038945

H 7.46052199642569

H 7.54830103862734

H 5.98096996403626

O 3.37946413415477

O 2.16832234429455

Cl 2.23615667099115

C 8.45063307433004

H 8.67444056819583

H 8.39956135875483

H 9.23985645817635

\section{2-_d}

V 3.68192619046694

O 3.79348578397051

N 6.40189517034208

N 6.25891995201833

C 5.51584807103927

C 5.69791841325095

C 4.32229553954805

C 3.81349811851796

C 4.68582148789766

C 6.01231383904640

C 6.48231129511535

H 7.47839693503403

C 4.35444544968194

C 4.65637672291073

H 4.25669897511202

C 7.62370270716902

C 1.38629725285685

H 1.51413643009887

H $\quad 0.33980293491747$

H 1.55190599993953

C 2.35424305135911

C 2.95838512515036

C 7.71497761791417

C 6.90142329905699

H 7.84814549845351
12.23091502439914

13.80574668555846

14.85902638800608

13.43213488107480

13.78497506706206

12.94187126218676

12.97656919800309

12.35377589630235

12.58252465150096

14.00418852353953

14.81161007163295

15.42709089824865

15.17152954977730

13.76673832915636

11.47653257004282

11.39275192998701

11.07181920136046

10.83842954223089

15.52539573867356

16.27630284289118

15.33903414421464

15.96570510558361

15.28764313381908

13.63988065738547

16.99614882143888

13.70531640144339

14.57851226654048

12.79736781001142

13.58434291143236

16.11954370673028 15.48140776415086 14.94384581928129 15.18571109116724 15.11135821520467 15.10744081921519 15.45676107694190 15.35403150272957 14.90137939459429 14.59151323127542 14.57086039728702 14.19361847458307 14.52503458802930 14.87686509255090 14.80407459764478 15.24816836811135 14.85781166851231 14.99891921669668 15.10923953832979 13.79221802441244 15.75774080738895 14.86793022380840 15.09064514076964 13.84717028256080 13.51952456033673
18.88700682150570

6.87733799608399

6.61342048891661

6.21533718643409

7.91329789098016

6.71582810495148

5.23716426581569

5.05219424192487

4.60590321174278

4.89532398725936

5.91956253413039

5.42487620950374

5.56702135041295

5.57625911692599

7.09852036605879

8.14016306100866

6.43901864277552

6.99575194024546

19.31364297266260

18.69392258976673

20.21681347894778

19.63994330392166

14.06671252153465

12.20638073697397

11.92966047506604

13.75870877982738

14.39239781392741

14.38314435322357

13.00304929193687

12.05899268752137

10.20424206324646

11.24095329694807

13.43160399597809

12.27794358664712

14.74498310112199

14.95290682261760

16.30238131860152

9.45483855650405

9.91157593456239

15.78491116563249

15.54382920990208

8.10246523406443

17.31111797642992

18.32849383731444

13.11667876239846

15.77884855269471

14.69277066446776

16.03798060480922

16.02482701878310

16.58798281185176

7.54724386422178

11.70612513098305

9.11881873460005

9.55983904430176 
C 8.76434318223297

H 8.68821815692458

C 1.86596223181641

H 2.02719869239684

H $\quad 0.86970450735054$

H 1.85752310487162

C 5.98780549285431

C 8.94783144708151

H 9.01050253704760

C 9.99180896423357

H 10.89073761296128

C 10.08188877195369

H 11.04996339472240

C 2.00143849355458

H 2.08986607076427

H $\quad 0.95351620505128$

H 2.63999421148553

C 6.57736660757056

C 5.30437684539662

H 5.03120367748504

C 6.81753091463398

C 2.73518102711973

H 2.79502614799761

H 1.73508000580002

H 3.49326664858528

C 2.13359108611462

H 2.80248808596925

H 1.08812802102033

H 2.32743798423532

C 8.23202249431418

H 8.20221077235458

H 8.79734749389897

H 8.80052358055779

C 6.10853799840562

H 5.09453692617926

H 6.68061424684931

H 6.00595726667061

C 8.92431041847913

H 8.77637426845629

H 9.66314739269433

H 9.36403258061576

C 7.58440540676236

C 7.07808942320708

H 6.14014202554639

H 7.83236942670689

H $\quad 6.89244930223967$

C 2.79757193663225

H 3.53438530196800

H 1.78949406914011

H 2.89628705092511

C 7.82961407378269

H 8.22504393604560

H 8.55826867128734

H 6.88694351501693

C 6.95456435427904

H 7.46928686164226
15.55126960728840

15.74084211960456

14.15713327129461

13.06285698154737

14.35658616902309

14.51471579073992

14.45386670311556

15.23457167821997

15.18399063074890

15.66742309671823

15.91426692697817

15.51185055605099

15.64024522164202

15.60739964211715

14.56023995137669

15.92137101260972

16.24004818955657

13.46582902779774

13.84120427871758

13.56739320285882

13.87958452864108

16.39943879523426

16.77003330659039

16.64789796061901

16.92302472111967

17.23987159002653

17.90008990786510

17.53383102499879

17.39369089791212

13.46562074959346

12.68034445927565

13.06427907803886

14.32354380675105

12.63183248916601

12.87626596917805

12.20932496397227

11.85016166143458

13.41222361482130

14.39443511247173

12.83949012692390

13.59311528644891

12.64598253100905

12.37763902166519

11.79412427858200

11.79910207362745

13.31850333102708

14.41593700320427

14.89935160812639

14.69590036497205

13.31989111515034

11.28348202225244

11.41566473420015

10.67545813458235

10.71303008175022

14.94783402238130

15.84614288168395
13.88261512780284

14.95648656652292

8.38546608157312

8.38213655716370

7.94571418136219

9.42863935961234

17.08818595191096

11.04259697835192

9.95227542386415

13.21735201373938

13.79490195586776

11.81625910226161

11.31724509921474

18.08090982636504

18.42592647758466

18.23856668259845

18.72559509874792

7.80864084110916

7.32947070212624

6.30745545670077

18.24892697889794

7.59579106382788

8.63099370319089

7.19246194241733

6.98401572960176

16.19717524259648

16.77999096230239

16.40954761805499

15.12422681917529

17.80374102706519

17.02698731209496

18.66498487674293

17.40065511820422

18.82623680605637

19.18797155819270

19.67479081136127

18.05232590147284

6.88072226476359

6.39693719417712

6.28758547873433

7.87771385216142

6.98202780895918

5.55282558993855

5.55581523060640

4.98804454289107

5.00410057699330

6.08228266746992

5.41370907864383

5.72580716060668

5.97174987009395

7.67251557803547

8.69509614806817

7.10198025233245

7.75181423962100

19.35947447168006

18.97365896531092 
H 7.53516262942305

H 5.96704255889600

O 3.52873529582143

Cl 1.36848494760113

O 4.19649078350144

\section{2-_isomer_d}

V 3.26615975192305

O 3.71979198530555

N 6.26189737942148

N 6.12038934948087

C 5.37595622346730

C 5.57045242564007

C 4.23141705384400

C 3.73519541813703

C 4.58582039671754

C 5.88508362662194

C 6.34150425875198

H 7.31673079534783

C 4.27294164619170

C 4.55908346923486

H 4.16902996026872

C 7.46384946234724

C 1.27888245758613

H 1.39870840033750

H 0.25328279464637

H 1.38506265135454

C 2.31163849472341

C 2.90369698514908

C 7.55509999889204

C 6.77556463808374

H 7.71065381517445

C 8.57538959544201

H 8.49214568523139

C 1.77560093627319

H 1.91142019882632

H $\quad 0.79453352884078$

H 1.75232910491163

C 5.85320926297384

C 8.75862970735814

H 8.81439157756041

C 9.77836094312768

H 10.65841098210401

C 9.86866803867516

H 10.81770880008772

C 1.98846016927863

H 2.01487443901933

H $\quad 0.96849313659757$

H 2.68593050216110

C 6.46396608371844

C 5.21807183098944

H 4.95933920913872

C 6.65454984024163

C 2.70364632968291

H 2.68389704045760

H 1.74114138980973
14.55448913318069

15.26685690769709

15.84019864870166

15.83091725348745

17.63064114131736

14.70186288065948

15.19991572834351

14.50825484843810

14.73517351111276

14.51505545295296

14.75863000274416

15.23117833840889

15.34796953441413

14.67500001160889

14.25441399046843

14.30824192097194

13.85797197883799

14.51279924672848

14.93532456484117

15.01702218764882

14.95673886436998

14.92778722162738

14.88593257900646

15.28227312377861

13.90658024979317

15.88645029444986

14.98348952935213

14.81011639309674

13.59868369395783

13.20403566540312

15.36517001886274

15.52780200280901

14.17061092526688

13.08800744255007

14.46833802155849

14.35258569387643

14.38968227502849

15.06947047583107

15.00843235534304

15.60056546189294

15.92472417308135

15.45523160610480

15.66841591894872

15.99830370053146

15.01540099092829

16.40591750513290

16.67773408321947

13.40804489539529

13.89736204202855

13.77191001276609

13.91048277833649

16.48896249870299

16.66687987339530

16.83144872104361
20.21626985421287

19.73622896731037

13.99566049219593

11.91655657762335

11.96181820563100

12.16180154754794

10.31543880209757

11.27118419329565

13.45454646597046

12.32114676115854

14.76970234813117

14.93496940329376

16.28274021385659

9.48773781412163

9.92021280250218

15.85527337037182

15.64995670594014

8.09435795768471

17.33795426160115

18.35844520997095

13.12539043196671

15.87640845442797

14.78200407380922

16.09406183812170

16.28623675352019

16.52017516664812

7.56753467147238

11.71586727648295

9.05912082323455

9.47278544601265

13.88317060725718

14.96148175271615

8.25057108565811

8.07302098338601

7.83371623458149

9.33693889245270

17.16551765823930

11.03712554604061

9.94644777444250

13.20339003473576

13.77192239546267

11.80111019136996

11.29447820806991

18.02283047927242

18.52925692332419

18.14513550193463

18.54802849388577

7.70310151004456

7.25623536170726

6.20192063430168

18.38802247638061

7.87168176518804

8.95900991719520

7.44613622884045 
H 3.51331318715159

C 2.16198433333896

H 2.90889110716279

H 1.15420748794888

H 2.28507540835799

C 8.02991758147406

H 7.93314486345147

H 8.57620465003974

H 8.65336909972264

C 5.86607239079851

H 4.87768394184941

H $\quad 6.41926720449148$

H 5.69599936825262

C 8.82829110086845

H 8.72917113893260

H 9.55880048930903

H 9.25127787663010

C 7.45847378061014

C 6.97767282903252

H 6.01848714430877

H 7.72298974231753

H 6.84199721090719

C 2.77977365006975

H 3.55297810575940

H 1.79268084584933

H 2.85134681939356

C 7.63346007063152

H 8.00705056731544

H 8.35177260825536

H 6.66755836873112

C 6.88130947147618

H 7.45387129427898

H 7.44346751671849

H 5.92408023865762

O 3.48097772270247

O 2.79366522673760

Cl 1.37674689331091

\section{2_isomer_s}

V 3.33201607774665

O 3.83978787927865

N 6.32442304009536

N 6.18406263995647

C 5.43857169695739

C 5.64055678431159

C 4.30495131990337

C 3.73708589279113

C 4.65900161591506

C 5.95183631113961

C 6.39048819853931

H 7.37556056127581

C 4.26734926175920

C 4.54456978753810

H 4.12170780037459

C 7.54548423229655

C 1.32517762645569
17.09109551010843

17.29474141909322

17.99292541821397

17.69563547138870

17.24935090752490

13.34230536378833

12.46988016439471

13.01281308753871

14.09875863190118

12.80057897997846

13.16162797466694

12.44803028350158

11.93745734023337

13.38756594792939

14.43194812661900

12.87205824802093

13.41200806089385

12.66971995788459

12.62109087919964

12.08187547194321

12.09439281462453

13.63500844527534

14.78572204186565

15.34822411214370

15.15371337559365

13.72062407427316

11.21674186713190

11.19141196954826

10.66226096428308

10.68120695671009

15.09879721908884

15.90128709743017

14.77666693266340

15.53388360735750

15.56110447107025

13.18028934599073

16.07589868247719

15.07789062662602

15.31482494462265

14.63745989813411

14.87116778183428

14.72022983323767

14.86607525248180

15.30392927675755

15.36052796235318

14.74939128095687

14.35268290766936

14.39376507235794

13.95922291605396

14.53548407868801

14.93389447271054

14.96927784357233

14.99407972466722

14.81506798884486
7.41842319605369

15.89304387838303

16.31521285921281

16.11294833770491

14.79892045126111

17.99350719339641

17.32259123801324

18.89643465150695

17.48279970449045

19.12217141134320

19.45594039098065

20.01405992720243

18.45407969873326

6.82016179073133

6.47321590804003

6.16734037232868

7.84027798619286

6.78919404684179

5.32727967862904

5.23126456010738

4.70307240370076

4.90976200131728

6.04414359191780

5.48775577568712

5.70983177358877

5.75492934318635

7.29056867869751

8.32938637990758

6.65602241699211

7.27271622957963

19.35211653200975

18.85295605685492

20.24989397316903

19.68862051839181

13.91655465705058

12.28296235849081

11.90001698257695

12.12603266584641

10.37302344276842

11.26085784889110

13.44671831013881

12.30490996974071

14.76334820900743

14.92894123689778

16.23652407236647

9.48946539036111

9.91623567219619

15.85423668421625

15.67214202169155

8.13853844911372

17.30146657906328

18.31000992040295

13.12409866038403

15.77793304913116 
H 1.49881626109375

H $\quad 0.27827891117165$

H 1.44395754129843

C 2.28670972673733

C 2.86946760973608

C 7.63535046637219

C 6.81612762943571

H 7.75999337483816

C 8.67551695720090

H 8.60241732291955

C 1.80148001591515

H 1.94978312296622

H 0.79229992121831

H 1.82987207461747

C 5.85537402930498

C 8.85617834836893

H 8.91929387160089

C 9.89335761968578

H 10.78929574713995

C 9.98239806390381

H 10.94622210208701

C 1.92758383157167

H 1.98338912597732

H 0.88939040504596

H 2.58205857487099

C 6.45373264052102

C 5.19290022058255

H 4.90025339152392

C 6.63078913740505

C 2.62640199363356

H 2.62592938519400

H 1.64290508319770

H 3.39963087248884

C 2.08263984656466

H 2.78482478821366

H 1.05427661518600

H 2.22369205364199

C 8.03675971225951

H 7.99645995065051

H 8.56118308195371

H 8.65406418767024

C 5.84959855686586

H 4.84192687482823

H 6.38634565095196

H 5.72837239395962

C 8.78265068651088

H 8.66686613504760

H 9.48769106717775

H 9.24872630066713

C 7.41948049307439

C 6.88641713110560

H 5.93128155476571

H 7.61418569343927

H 6.73161438409867

C 2.70832384361962

H 3.44623258873030
14.72789048269476

15.13693554341943

13.81415671937091

15.83283642637494

14.95735649988504

14.84333716774006

13.66351697289278

13.27326537744825

15.32727025475701

15.50338715292327

14.09984663803655

13.02677678122837

14.38433899554085

14.23946103572924

14.42795091310963

15.02144113299316

14.96603944894623

15.47839836469164

15.74440261011380

15.32805259347870

15.47949734473869

15.92329113787755

14.94141742592821

16.28504178949012

16.63149845585663

13.42925662648986

13.89408050168440

13.72596000579587

13.92676661333483

16.45829501578234

16.67286968682084

16.75962376780812

17.08209206823467

17.23540809267648

17.96645351226567

17.58513394331807

17.22465479735697

13.41979388001655

12.56668723259841

13.07582831753412

14.21548014839310

12.76470498874398

13.08095994127403

12.39298093916375

11.92350289103481

13.39519576600541

14.42870457831291

12.86563996728825

13.44554704515019

12.66542655900367

12.56569199993096

12.01307181245746

12.02393256699770

13.56266713686908

14.73776652103768

15.31846146041214
14.69419342549854

15.92676942927312

16.23015189957490

16.44089898055717

7.65151006506148

11.71864473390868

9.05539655806565

9.44765542505551

13.89199988162298

14.96819575155554

8.37537735443282

8.15875890524363

8.02548108581890

9.46713569465639

17.14818669386143

11.04401012443285

9.95406591658081

13.21853570793818

13.79097785583008

11.81669522127225

11.31742347617679

17.93666379964897

18.44063916081586

18.03889569156868

18.47695732191372

7.71852897092587

7.29403838075326

6.25567693885429

18.37840031927679

7.94196608989171

9.02285619534433

7.53763528067192

7.45775966371112

15.81829643044916

16.25868694904439

16.02235266455423

14.72545038103577

18.00965737693842

17.30859772730414

18.91870813763604

17.55376764651361

19.03554768201793

19.35657902121157

19.92774652571498

18.33009698428447

6.75812771018620

6.38560640405737

6.09138596359077

7.75840470075814

6.79508742526945

5.35418338956202

5.30650510019614

4.72429032112580

4.90407805951656

6.13471459834685

5.55161016642032 
H 1.70231839381197

H 2.80122508366513

C 7.61677347149062

H 8.03449853961017

H 8.31215041730158

H $\quad 6.65563384120823$

C 6.78086022202683

H 7.33808213322923

H 7.32930437671314

H 5.80020430620830

O 3.60357030612008

O 2.83322844081399

Cl 1.58284387885845

2_S

V 3.63749098273354

O 3.91010940885910

N 6.44740404006923

N 6.30737423347707

C 5.56333221484108

C 5.75837407337832

C 4.39523970150841

C 3.82504860948795

C 4.75000984380719

C $\quad 6.06800943630857$

C 6.52543013148758

H 7.53012708475004

C 4.35593692548369

C 4.64960460478920

H $\quad 4.22447117836857$

C 7.67567709995560

C 1.42582189076230

H 1.58750874692519

H $\quad 0.37110513615790$

H 1.57749435516498

C 2.35981233008638

C 2.94877395088646

C 7.76504740194292

C 6.94487624168441

H 7.90541490073600

C 8.81908680753120

H 8.75155470408715

C 1.88590947918210

H 2.03804953052231

H $\quad 0.87592046176407$

H 1.90816635908808

C 5.98689029055776

C 8.99821919123779

H 9.06497314716844

C 10.04627712730760

H 10.94969105883051

C 10.13471871364249

H 11.10566651258082

C 1.97051388449118

H 2.05329016895827

H $\quad 0.91829157751880$
15.07158949408788

13.67307924861483

11.23287793973793

11.24080770555333

10.66292794588387

10.68998674363681

15.08628188758932

15.92950438463008

14.74653665199910

15.47003275584719

15.65233116265363

13.58101329227818

16.47909978486135

15.96848909623233

15.44621183927198

14.91480395550266

15.14799179221767

15.08863876440360

15.06559926795217

15.40940595518272

15.34606075847126

14.85953592690749

14.55459941390570

14.56081600580183

14.18570900298600

14.53000463733597

14.89431145934409

14.84330276761687

15.18909947951644

14.82223971077349

14.91565459394419

15.08343893057249

13.76667932378777

15.75247087992646

14.88770011927461

15.03860115146226

13.83454748093476

13.50275848892469

15.46481495367748

15.65584496291211

14.14856952300273

13.05502890510212

14.37022753083976

14.45873041648221

14.47274140125019

15.15975015048743

15.11967274789236

15.55679386327845

15.78360628786157

15.40673840209750

15.51900105036089

15.63545172846012

14.59795732089141

15.94663267235704
5.82532750377935

5.85398101918719

7.34432562404293

8.36659161444119

6.70080965933513

7.37933861916355

19.39093352904784

18.94493993299155

20.28873724435710

19.72181936813606

13.85416861105400

12.23571424885407

11.87274788186404

12.06901373552894

10.32724495637410

11.24880242890091

13.43971299858279

12.28600742030346

14.75204347632836

14.93793714650495

16.24860694490242

9.48970540124700

9.92377957542817

15.81918336346118

15.62011081336277

8.15849368979058

17.28695882584201

18.29376005622266

13.12378353700114

15.67184726846772

14.58563602169687

15.87545395342232

15.96208275790408

16.48329586466359

7.64911986331810

11.71752457418525

9.09780335730050

9.50024663019608

13.89630641661823

14.96982761621972

8.49761802579182

8.44777927243828

8.10648519009110

9.55500086538495

17.10713229292843

11.05093856115651

9.96098394826251

13.23032542281704

13.80782364189907

11.82889967392215

11.33315389333338

17.96964779741849

18.34151624144940

18.09298936590659 
H 2.58666577466634

C 6.57671725231221

C 5.29352277723461

H 4.99694988742111

C 6.78591154316567

C 2.73197689466526

H 2.81098847156753

H 1.72520519670945

H 3.47495639432070

C 2.14877868451621

H 2.80007696403972

H 1.09945958523040

H 2.36257414001933

C 8.21539254772761

H 8.21785413204722

H 8.75505510748583

H 8.79347934612708

C 6.06336172882642

H 5.04064201535879

H 6.61679430720213

H 5.98567284340903

C 8.89057435659980

H 8.72586058466380

H 9.60743388224802

H 9.36881905264249

C 7.56025430846344

C 7.00941779783114

H 6.07680330085247

H 7.74867580174276

H 6.80713975551580

C 2.75393416412789

H 3.47149202001905

H 1.73833197879037

H 2.84864360091631

C 7.82749563820628

H 8.26229015308138

H 8.53566270823568

H 6.89116280083411

C 6.87792979729373

H 7.39306961783117

H 7.44163608231549

H 5.87924377132296

O 3.66769107319848

Cl 1.42196824459045

O 3.97431937650406

\section{2_isomer_t}

V 3.23782586235472

O 3.66884483096868

N 6.20473765720031

N 6.06509686895158

C 5.31406138007764

C 5.54475765570954

C 4.18951125164393

C 3.71066284580535

C 4.55219955194237
16.28865923648760

13.48178682218250

13.86425815451838

13.61259090704223

13.92900834285995

16.41812550192499

16.78133160109250

16.67611226252932

16.95555803369767

17.22465035583050

17.90020502934354

17.52015551441966

17.37194421159229

13.51774839007516

12.71342487736329

13.13865323690138

14.37200368873199

12.68884278158007

12.93247966634922

12.28677940577503

11.89046109301441

13.46342391007115

14.45688655468246

12.90936092846015

13.61609179431508

12.68415908823660

12.44343086275021

11.85185955872023

11.88018843162028

13.39247466802489

14.47120826257622

14.97374554454401

14.75814611617004

13.37904297642217

11.31134775909515

11.42120221778604

10.71898293859276

10.73396448261750

15.02510174626609

15.92227921739427

14.65425053305732

15.33760626221139

15.78369638172989

15.57730535662772

17.51439657551386

15.03511162570453

15.23397321944831

14.61075442431611

14.84623095019217

14.66468561388319

14.83116358356253

15.27603918483765

15.34808240468614

14.71269513681396
18.61412367626694

7.79081626107141

7.35061636489185

6.33052920660822

18.30343199951822

7.74069306978521

8.77719969120277

7.36468027580695

7.12412249929002 16.05224027604085 16.63567796039459 16.23415069268198 14.98222605603515 17.90726298776942 17.14953073469147 18.79314264470950 17.50967453518980 18.87975372899938 19.21612198439638 19.74829986093963 18.12035229523580 6.79315577056838 6.33919374869314 6.15966603943277 7.77722092619895 6.91574509326129 5.49840698852319 5.51508744831478 4.90159897114431 4.97033201483791 6.17823470114400 5.50434715213873 5.85347190568148 6.03819819611155 7.57652579543284 8.58579331359822 6.96811416748036 7.67552064748688 19.39104575393403 19.00370484289386 20.26687567481305 19.74239804262415 13.89905179562639 11.96025570571083 11.94683761624974

12.12481031070296 10.20727349282116 11.25605352907044 13.43997380839254 12.30183030641350 14.75777883838610 14.98291997567461 16.35121784339551 9.42602691629303 
C 5.85464822618611

C 6.33605702764862

H 7.31051199927073

C 4.25819100349347

C 4.56487438055859

H 4.20486644236023

C 7.42588919376461

C 1.26403041563043

H 1.38675609798676

H $\quad 0.23783263787380$

H 1.37103991910005

C 2.28605176277466

C 2.89818241080074

C 7.51531179677826

C 6.75463390941807

H 7.68096518906176

C 8.54183014777706

H 8.46566892895880

C 1.77181886742707

H 1.90153276261391

H $\quad 0.79327204634975$

H 1.74993741237234

C 5.86619631954493

C 8.72160095913535

H 8.78115943675435

C 9.75043365417520

H 10.63575787813370

C 9.83901941454306

H 10.79206991088542

C 1.99620183634271

H 2.05419510842562

H 0.97104521200453

H 2.68604592010071

C 6.46196432958817

C 5.22286578015988

H 4.99596393770392

C 6.69415235283729

C 2.68127905707537

H 2.63958351197729

H 1.72452570754601

H 3.48864534886659

C 2.09969858455520

H 2.83550407836022

H 1.08958021573460

H 2.20144618504030

C 8.06950284527986

H 7.97722336044153

H 8.63033474678025

H 8.68054938499324

C 5.92261561277884

H 4.93936182083822

H 6.49930425342105

H 5.75045534693531

C 8.84108062375168

H 8.75927517683243

H 9.57614605614477
14.31752897091206

14.35906950148213

13.91997072155900

14.50603842789194

14.91984325389586

14.96180175533998

15.01675649955351

14.87932038159809

14.82178476275324

15.23008197551209

13.86229590877930

15.84958309114465

14.94458444816591

14.86503688039636

13.63592071453340

13.24698287667376

15.40528761700654

15.58398585871948

14.12950147041623

13.04817360443765

14.43290281183082

14.29564808931373

14.39388748394633

15.09719876930234

15.04226890957559

15.60858324975781

15.91682792062714

15.45716191336821

15.64965182908956

15.90607153736041

14.90968751516598

16.28449062180428

16.58836236789934

13.40132624125744

13.87378483980164

13.71012333215034

13.87964964814407

16.45951655321606

16.69765158886801

16.77123053653996

17.05199338294734

17.27677699905395

17.97483286705492

17.64492694025352

17.29438547339058

13.34441355153941

12.49194900153356

12.99033990900757

14.12671215255681

12.73437825342523

13.06985740616108

12.35671696775973

11.89323039558911

13.34835783684416

14.38338281996506

12.81118192657579
9.91448347639660

15.81582079485441

15.59085930089837

8.02242682498633

17.36393000038824

18.39628633561708

13.11146958483389

15.98420216516969

14.89181539106233

16.19715129640007

16.40291682402339

16.63133755315046

7.45987208104796

11.70838365963815

9.08965430605049

9.52352933937389

13.87136883351697

14.94707409111092

8.14719787321057

7.96027552130411

7.73265070446273

9.23511198036048

17.13577578828889

11.02700121441092

9.93668933733671

13.19204740206151

13.75976791994005

11.79205181081095

11.28630005946909

18.14356160358619

18.61882266773593

18.30054811501919

18.67366096179956

7.73420116291068

7.23447532168087

6.17903117648784

18.32054095599909

7.70807134145774

8.78264577584462

7.25093791290371

7.24007877402660

16.05564369064627

16.49526609229089

16.31176428903945

14.95904255732850

17.88298546730887

17.18626290121858

18.76582872749236

17.39674460418649

19.02009123981336

19.39245162755289

19.88419259221893

18.32546707829697

6.91507673430394

6.53792006682914

6.28835357300076 
H 9.25031860922448

C 7.47013935040983

C 7.02366526838869

H $\quad 6.06900802821550$

H 7.78380694837417

H 6.90539235856944

C 2.80745786670696

H 3.58019353560713

H 1.82289936746297

H 2.89599666573635

C 7.61897042521671

H 7.97642601115099

H 8.34499255987382

H 6.65225180468242

C 6.91588127755885

H 7.46637870181391

H 7.50334008299748

H 5.96169973459815

O 3.41323204769295

O 2.45187036783515

Cl 1.98378764533644

\section{2_t}

V 3.57292186627723

O 3.75540775558408

N 6.35062122669788

N 6.21041816556436

C 5.46442704076232

C 5.67323036961128

C 4.29622313755417

C 3.81163876185632

C 4.65775501663743

C 5.98323712532858

C 6.46790377287749

H 7.45417577718735

C 4.35628453188606

C 4.67191481470113

H 4.30494598204038

C 7.58337474092552

C 1.37125531746369

H 1.46983950843869

H 0.33849155678455

H 1.51744020987061

C 2.37208935570543

C 2.98147674016072

C 7.67283009994493

C 6.88524543565939

H 7.82392015666457

C 8.71643260539859

H 8.64486447121669

C 1.87284615817503

H 2.03383015436815

H 0.89024305960321

H 1.83026924826834

C 5.99109240057806

C 8.89594697069192
13.39022759605361

12.63192451887831

12.53812471594832

11.99324692582680

11.99038096651511

13.53638449540129

14.69447866728614

15.25236391991368

15.03701762634550

13.62236351461176

11.19679598803932

11.20018987069273

10.62186801334190

10.66353315369718

15.03959583204078

15.87115700172715

14.68799323325954

15.44212141911490

15.60323694878929

13.65164044844422

16.95469514517991

15.90804461555780

15.39258570462326

14.88425453825057

15.11595713121066

15.03581287763316

15.04055062778813

15.41845926174303

15.38162633254249

14.85277205103175

14.53324573177413

14.52258908610192

14.12282267756829

14.53668810980034

14.91628430346930

14.87974375700617

15.19088770693496

14.88348773580611

14.93199232324999

15.17765665881363

13.83673396739192

15.82171057978299

14.90102912354322

15.04187998047381

13.81381804586919

13.47102817730326

15.51215527121426

15.70005026439394

14.12370914491328

13.03371786502060

14.35816266211736

14.38952782331554

14.45064329684783

15.21068178081864
7.94012687934167

6.86770054522439

5.39723318487407

5.29063975096045

4.81267045977085

4.93921248334619

5.94213614599365

5.38183374653387

5.57867363638252

5.68796418510222

7.42890263034227

8.47360464987995

6.82519311322978

7.40606282592538

19.32138299874554

18.84635562534577

20.18919200769488

19.70283674656891

14.00717712408149

12.20317206399199

11.85440609835962

12.07145439687799

10.16811537344813

11.24493486334130

13.43569131275798

12.28394003085023

14.74661033340948

14.98953191615752

16.36019054571205

9.42481590128141

9.92007509861686

15.78364387843165

15.54215107392436

8.04017867205530

17.34957998839631

18.37955445956167

13.11627455817408

15.94917440851977

14.85288617468432

16.21182418683196

16.27289479846583

16.67102148201966

7.45819956535228

11.71104741959386

9.12602574856243

9.56985389767731

13.88293229910989

14.95704432377107

8.21344809610862

8.12493475918007

7.76437580512218

9.28189421209494

17.09743318550121

11.04022135962519 
H 8.95982246717437

C 9.93932860750156

H 10.83554577118234

C 10.02781967658098

H 10.99181789517944

C 2.07653022788630

H 2.15532641618325

H 1.04219621619069

H 2.74780529654450

C 6.58246487623241

C 5.32536862710487

H 5.08980834505523

C 6.82777504612783

C 2.74173637646087

H 2.73728163006082

H 1.76332750412355

H 3.52103661916410

C 2.15679693560812

H 2.85824249485294

H 1.12838894665015

H 2.29465873739263

C 8.22519127383005

H 8.17029244363304

H 8.79120950303548

H 8.81118127188504

C 6.09219871771676

H 5.09343901026127

H 6.67312456823054

H 5.95817762998736

C 8.94502509694996

H 8.82019156637086

H 9.68569032720800

H 9.37210401091384

C 7.59866977828376

C 7.12539872542901

H $\quad 6.18547136419562$

H 7.88969033507256

H $\quad 6.96767764747390$

C 2.88426855280752

H 3.64211450849920

H 1.89080432914506

H 2.98904296310013

C 7.80917845590554

H 8.19073786627011

H 8.54079391122564

H 6.86124476286200

C 6.99981654213572

H 7.52398890782875

H 7.59216582502942

H 6.02882440406457

O 3.49851105616995

Cl 1.41535582671618

O 3.88134557002204
15.17036538069795

15.64945286458027

15.90870454912023

15.50132558500543

15.64725873381539

15.75699841224867

14.72863984397690

16.09889446298990

16.41226697183767

13.47217003659733

13.87574782499375

13.63396871757783

13.88007236635359

16.42713761422723

16.75175190295951

16.68371141793376

16.99281845956915

17.28579861913049

17.96637182782665

17.60296658816507

17.39440186321297

13.42762288612120

12.62593078067222

13.02970029741845

14.26516383156003

12.66015926426906

12.93257146604601

12.24122204790485

11.86621975360882

13.42694265998586

14.42398995648410

12.86369013779998

13.56733235869270

12.66506814625686

12.43440556796109

11.85580694514358

11.86121443933595

13.38636529026596

14.53340592381277

15.05898670805471

14.83006454724491

13.44639169392193

11.28714085793975

11.38861454903389

10.68778596232565

10.72241850995342

14.97019394609702

15.85316926970116

14.57666259972408

15.31000696935431

15.77512978926202

15.13297814181626

17.46767544550006
9.94982935460550

13.21299812097804

13.78797458721812

11.81317488100388

11.31272490912251

18.18221767623244

18.58001784612566

18.36132591125242

18.76733952886411

7.79803451105150

7.28415400092185

6.24592850093848

18.24997737213497

7.58482927321077

8.63627453618274

7.13974753112532

7.04237518407037

16.21049901260438

16.72660557821618

16.46150488510823

15.12412668385575

17.78953743687494

17.03121098830871

18.65015147809428

17.36889860241515

18.85532567751272

19.23784553217809

19.69731081012976

18.09935054944781

6.93202220031227

6.47341564484577

6.33543746896708

7.94092745525493

6.97573260948763

5.52897410942044

5.49094963263839

4.97500843203235

4.99126251627978

5.96460149556149

5.35494279188639

5.58528048853849

5.79349709869237

7.64892363979844

8.68012824848083

7.07649424025305

7.69465839550257

19.33545262170150

18.92838781302735

20.18171269003926

19.73508125068079

14.04360049567043

12.00208155444907

11.93819402147767

9_q

V $7.52693884165833 \quad 5.30478316760423 \quad 4.83393130157467$ 
Cl 6.80892915313863

O 8.41098599709191

O 6.70064732005897

O 9.34237352197695

N 9.34832662379617

O 5.87750684554752

N 9.46343860249709

N 8.23256505096400

C 5.95718025290900

C 11.63906605468324

H 11.63032885269996

C 7.34536861152994

C 10.46400630457422

C 8.09974773456147

C 5.29740300256925

C 3.95716116826047

C 12.76234283453130

C 11.54948843982716

C 5.19132882810865

H 5.63520907776831

C 10.38796266169854

C 12.67936779826740

H 13.55465908525549

C 3.30051875208755

C 3.86987457790299

C 3.09210990281374

C 14.06131281134952

C 11.49135138456166

C 1.90157244951924

H 1.93458384709972

H 1.47870658788414

H 1.20062610842998

C 15.22210144399694

H 15.03162537696398

H 16.17207546522616

H 15.35856500757185

C 3.29443868277109

H 2.27564757365369

C 5.50126699086819

H 4.66575034475400

H 5.68549501595124

C 14.36337085743529

H 14.47593837981029

H 15.30244305115011

H 13.54849144567626

C 11.23961059362093

H 12.02049833566057

H 11.27042259778478

H 10.25260432377391

C 8.08141189602100

H 9.08791464950610

H 7.46958551439831

H 7.60755401943497

C 3.84983560616871

H 3.95454384371414

H 3.30436769330104
7.06664419746982

6.73445148501441

3.91827973384275

5.10552558238367

3.21813420069351

5.37489648085421

2.32573703002869

2.32834527296787

3.41418949212359

2.69061555547839

1.99297732909270

3.18328676820189

3.43001860074752

3.79526521133200

4.60695214357997

4.93889040326189

2.83814476078107

4.50786312923443

2.54886843048907

1.60491786714263

4.38644892248060

3.74653697828771

3.84534724983952

6.24729133600797

2.82741397104606

1.82246625013560

2.05442861786176

5.45683629488551

6.43594302482852

6.46314632889753

7.39640327020972

5.63822245611637

3.05276932233578

3.67944628167974

2.50909649519620

3.72696720311169

4.04626056080532

4.28588265197911

4.13524119271272

4.34769883878216

5.03624833982333

1.19013055187159

1.80646472326152

0.62378323507845

0.46702424611775

6.91193464196806

7.23268269716383

7.59534842778082 7.00762575522070

1.61738418258784

1.47217532489069

2.21170328877978

0.62764389340020

1.59434296513363

2.54064393852824

0.87451715547000
6.31374777027479

3.51747475486025

6.17019028628970

5.66466303738903

3.50507775403560

3.71025511559335

2.45680924745777

1.91542876971426

2.28883794919320

4.08761481872843

3.24749037674596

2.59657293122226

4.32597072867114

3.61665209341860

2.85313693745798

2.40701847154837

4.90599807366866

6.29103152896327

1.47258434133370

1.14354581066274

5.42929270415179

6.00123095792808

6.65021650749931

2.88367500583779

1.10727364370189

0.24054084024008

4.67510301590160

7.50177176455011

2.26560426681837

1.16101352460451

2.60943568837715

2.57249421443239

4.44789745374912

3.55846805019386

4.29058697890359

5.31123865055528

1.56574712763989

1.25457629009087

6.97460758935769

6.28621289049797

7.58608025713773

5.92313411292159

6.83196768561093

5.78104321547074

6.10543661172806

7.03238508332523

6.31852185316730

7.90089458035595

6.55471377112333

0.66229512641775

0.24038416155843

$-0.03610386470569$

0.80250215418794

$-1.08931524273945$

$-1.64950459731197$

$-1.72729534362639$ 
H 4.86296570633357

C 13.97170021233947

H 13.17529384915509

H 14.92721958445208

H 13.77451936669682

C 3.14437070823642

H 2.58408142003965

H 2.57465209698711

H 4.12304188633038

C 8.30103687547349

H 7.23908327640928

H 8.89402919937814

C 4.17523233127891

H 5.16526825857753

H 3.68676189615339

H 4.30356771634034

C 10.35000950586680

H 9.36759588176217

H 10.34384116078553

H 10.49873361138881

C 6.77030503689572

H 7.33799494881319

H $\quad 6.82540726379572$

C 7.32181068472046

H 8.41432068352432

H 7.03639487953686

C 12.80873075085879

H 13.03816859280568

H 12.72314294151795

H 13.66787614210011

C 2.97694950030953

H 3.96963259827916

H 2.42436016031766

H 2.43900882518160

C 5.33181238945484

H 4.87175101238647

H 4.69209323454707

C 8.91639056191979

H 10.01051562136530

H 8.41528952963297

C 8.80792448398198

H 8.27631470040158

H 9.88670753631246

C 1.67087965667979

H 1.68578936739161

H 1.06388989294299

H 1.15456336711230

C 8.57340192723485

H 9.19317576789044

H 7.51120860326595

\section{9_s_UKS}

V 7.53599768793587

Cl 6.86433081161813

O 8.36747437175672

O 6.73657974847685
1.18918711212143

1.12630734023791

0.36985653590676

0.58800019887612

1.69240050117960

6.25020265125560

5.35827838664308

7.14438874319630

6.27754523744054

6.65540575547872

6.47826291713171

5.79142466870144

7.45371959093861

7.40940905274118

8.39734692577654

7.47493330952078

5.01805378159171

5.10571974062943

5.66315884479032

3.97381117547409

2.31444174374258

2.85997091563554

1.23569485102975

2.65501425513124

2.79597588156943

1.90605399365113

5.43712619093561

4.43219919851724

6.12410915757986

5.77388453130592

0.47989448773340

0.05533327746142

$-0.26246070490615$

0.61530532353957

2.84790018664401

3.03748773857776

2.12540449915788

8.03475008751911

7.95377396333712

8.28047931733668

8.00465047162841

8.31942071577200

7.95034328718954

2.31234239311021

3.26159632212134

2.46270679859294

1.56117410323188

8.94030228344612

9.85246367420922

9.24265312896606

5.26632023871845

7.05205616254341

6.65119845252561

3.90344781137958
$-0.91936205232420$

3.45061108948581

3.56787547757791

3.31811627009372

2.52277854945144

4.42605753974403

4.76286774589251

4.73991384725402

4.93108165787945

2.07375989432694

1.82495650624021

1.72304731565063

2.45550476716660

2.93602376252049

2.76036140977442

1.35750794698848

8.45553888214967

7.96489993579677

9.35335456570621

8.78770939659667

7.87942781587779

8.65573784250515

8.10356661514754

6.49932393734219

6.46920666139617

5.73281017419103

8.30032156062467

8.69962556015450

9.16076972441677

7.69176094093283

1.00173955217040

1.23313247964211

0.39632613562561

1.95684476628206

7.78390695488801

8.76836217824923

7.24370615422425

3.92305420301075

4.08358651684219

4.87497887547832

1.55038675746861

0.63632578987760

1.31436143759525

$-0.09354233234997$

$-0.65834073218024$

0.81686912639988

$-0.71754311892345$

2.74788141734307

2.71685806166973

2.80589660766859

4.82510520212553

6.23386060541083

3.44979204455561

6.22457677809548 
O 9.22705904946877

N 9.33198683841866

O 5.98780067720816

N 9.41115263626873

N 8.18563367137431

C 5.93807298758266

C 11.66493293928973

H 11.68385060353098

C 7.32563586657488

C 10.46422692631326

C 8.08861318958582

C 5.33113285183572

C 4.01491192003344

C 12.78043573175501

C 11.47484864818566

C 5.16293580527010

H 5.58479435520263

C 10.34883395725038

C 12.64678996608981

H 13.51077006455856

C 3.39792003249625

C 3.85176703741726

C 3.05509652728913

C 14.11838741996494

C 11.36165919921672

C 1.99734308564260

H 2.01903068697296

H 1.60243163269109

H 1.28074787878299

C 15.23074480232698

H 15.00783141661249

H 16.20632101615605

H 15.33600164089175

C 3.32049542664877

H 2.31100364719207

C 5.58316044116890

H 4.72542602995562

H 5.81434880834240

C 14.46604155352376

H 14.55033200250134

H 15.43095206216590

H 13.68664530259308

C 11.03669574153978

H 11.79853992346805

H 11.03363255036886

H 10.04495435185259

C 7.99199984471915

H 8.98384074505224

H 7.54785287409109

H 7.33931646838529

C 3.81366702540816

H 3.94545317477279

H 3.25727612109750

H 4.81643301967783

C 14.06685150868238

H 13.30428552044897
5.00630791988779

3.11526473403186

5.20023204874094

2.16314381584830

2.15263574144456

3.33384074566141

2.68107671398239

1.96750812881585

3.06149313041637

3.38086729205290

3.72468084961399

4.49529546915900

4.89762133185921

2.89764295509864

4.54120561875219

2.54615333869138

1.62486737451131

4.33895845553227

3.82373753120338

3.98410135377850

6.19997061762268

2.88706978448677

1.97180087873351

2.16990090972856

5.50538356081412

6.45743392202198

6.54693830454701

7.40683288326773

5.66017513612165

3.21249179674355

3.80525745376439

2.71236461658206

3.91655886690161

4.07741941636654

4.37555019881895

4.14182110782793

4.39073242653432

5.02070611195019

1.35374411819259

1.99958494797543

0.82870102267720

0.59826926637113

6.93735088970072

7.28544832792298

7.63637730592744

6.98051754509499

1.24467623833087

0.85981463382127

1.78030205095052

0.39912656957434

1.82671806900591

2.81036541283666

1.17053661311872

1.38608833570718

1.20548383125641

0.41860946465376
5.57357743931808

3.52056414453774

3.77881135651244

2.57105229229962

2.03070526942633

2.27802789835465

4.09675881716343

3.26965980865928

2.62266516407807

4.32725859063476

3.61398659807705

2.88116170256284

2.48644824793603

4.90926634190057

6.24392510450309

1.39623649600473

0.98845548637646

5.37883886308081

5.97396595873905

6.62625722803953

3.03532626953616

1.04123816038628

0.09247798068441

4.69641810968681

7.44042530739665

2.44615428527572

1.34474812924362

2.84918638648856

2.71531765794279

4.43560887888210

3.53042984939990

4.28960117970127

5.27953825206573

1.58392519315266

1.29286107792341

7.08267074250373

6.43591604613209

7.71041844302458

5.96354190393035

6.85497942170333

5.83534816480077

6.16904706818226

6.94987968572204

6.22838807552697

7.80654108768026

6.47415592181313

0.91445714444978

0.63618230313594

0.05943332001162

1.19357006036746

$-1.24755663384985$

$-1.73271014240072$

$-1.94250617623732$

$-1.10418997663043$

3.49790449351722

3.63914619625105 
H 15.04451200976491

H 13.84234424012811

C 3.26020941358844

H 2.68516568326818

H 2.71972003112844

H 4.24613769845625

C 8.19858823871114

H 7.11919250569764

H 8.72251220894026

C 4.29848341736566

H 5.28953912877511

H 3.83613589660544

H 4.42363844068184

C 10.23710134553017

H 9.25760753476762

H 10.17625550594189

H 10.44386699741968

C 6.84378198487373

H 7.43616309052001

H $\quad 6.89157116409709$

C 7.36734697767428

H 8.45899763063427

H 7.06829584162454

C 12.67192293887508

H 12.95096136326120

H 12.54407595571956

H 13.51625348719163

C 2.89508158578354

H 3.87433586212598

H 2.32927670073134

H 2.35323041817109

C 5.41063027189421

H 4.97316367810393

H 4.75007320999344

C 8.95405088579148

H 10.04511295957962

H 8.49338533206777

C 8.77103904493700

H 8.23190743647586

H 9.83668730228707

C 1.65124732747828

H 1.69928559217526

H 1.04409542347832

H 1.11528695862428

C 8.63065983698677

H 9.30385461881241

H 7.59128999755591

9_S

V 7.63891514005946

Cl 6.83939185462997

O 8.33517804253266

O 6.84170630991579

O 9.30373727737341

N 9.40870185042173

O 6.04886912581628
0.70560823659744

1.73698212238308

6.13045633417314

5.23553414203216

7.02156692853897

6.11213622051586

6.53203609515952

6.41452281245340

5.62076833524192

7.40385643244033

7.31754739119477

8.34467898149096

7.47284494863234

5.02032835912078

5.03524714001779

5.68403118789947

3.99464061626414

2.29378339212273

2.82410635905840

1.21227554219409

2.64049462946521

2.78229207156358

1.89394375300810

5.56558173877766

4.57941595065595

6.25858533572242

5.93845908268225

0.57817818543680

0.11020545256091

$-0.10183105886207$

0.65390599269509

2.84559699812920

3.02540582683847

2.14201350977058

7.93482859784717

7.79861336691529

8.24715394006020

7.82697560844898

8.14277030096910

7.69718142843200

2.52809832030537

3.51760686247481

2.62693718137874

1.84380089765311

8.81689733615119

9.68703928760558

9.18680997558112

5.21309167561456

7.06156689020761

6.59726376611751

3.90549817852216

5.04895063012537

3.06027607911082

5.08732583768753
3.37564614597462

2.55572040160848

4.57570896994633

4.87721644663334

4.94501124137638

5.06616181464191

2.01278323961116

1.80939123120227

1.67429237438995

2.66012545879403

3.13334156904998

3.01131170450900

1.56377244480868

8.39051543802384

7.88604783982871

9.27264661506658

8.74790820284932

7.93027618038508

8.69869215473799

8.14222406540053

6.54070676782404

6.49311227536042

5.77728544463482

8.24841605902196

8.66206633398527

9.09887976218750

7.64041970510641

0.74393292511325

0.94915848040287

0.07994168811937

1.70350273694849

7.87516808090716

8.87170858295057

7.33521398331392

3.79363688746086

3.93334546210716

4.74541429215087

1.42709192053885

0.51791479719085

1.16282116028249

$-0.20870848579447$

$-0.69763680435003$

0.70880993143005

$-0.89061889054061$

2.59508935507036

2.51323665505349

2.67057104889267

4.82051282637724

6.14609471197810

3.39877146964091

6.25239005433687

5.51947533270068

3.59455647564382

3.86007423224160 
N 9.49085043515203

N 8.27939370245441

C 6.04260926797453

C 11.71662963098144

H 11.72376565076599

C 7.42707896426856

C 10.52657008716795

C 8.16693609473992

C 5.39859373926916

C 4.05214555231722

C 12.83573233074858

C 11.57067279326846

C 5.29810172720896

H 5.75524726523393

C 10.43857087268630

C 12.73182904310536

H 13.60589207760709

C 3.37415735113467

C 3.96896779226414

C 3.22026102833905

C 14.15698942515209

C 11.50483583034815

C 1.93230100058017

H 1.89643039593067

H 1.48565238038122

H 1.29069164475642

C $\quad 15.29014027422645$

H 15.07448274020903

H 16.25307480062495

H 15.41645821173320

C 3.38056449589939

H 2.34912714139674

C 6.71126938414680

H 6.66157740416238

H 7.61644716779470

C 14.49064985893450

H 14.59245691601137

H 15.44298394011697

H 13.69537223015066

C 11.18756406206197

H 11.93476480032012

H 11.21278555137052

H 10.18520254244128

C 8.08278721621577

H 9.04954117499566

H 7.76813303712874

H 7.32557520967179

C 3.97057221450392

H 4.02765265371880

H 3.45230612453019

H 5.00357495355642

C 14.08061615233076

H 13.30029470742912

H 15.04635744982173

H 13.86655403503927

C 3.31502461699375
2.08265187013259

2.09992898155855

3.31025977343168

2.59381403570330

1.81838358996367

3.03320097043164

3.33111212439473

3.70481291847127

4.40267354357181

4.75733907978513

2.85923989451468

4.64318539295091

2.58904121323526

1.74069866855866

4.36027320685302

3.88258701806944

4.09170842131769

5.93334884313293

2.91175807728415

2.07809776186688

2.08554077260580

5.74608008969018

6.15284899594082

6.39175156098237

7.00435847720888

5.27122888550323

3.07585745939291

3.59033896678141

2.54224254385473

3.84945083354811

3.99842588834669

4.26639524400436

4.17671751602076

5.27318540604380

3.78852783627091

1.38226811003787

2.10561464299046

0.82591665210059

0.66675943323091

7.11061375586720

7.35393944800888

7.91145695195736

7.10877522074573

1.14825502604974

0.65256497078741

1.66820229546360

0.39386437304235

2.16516377073116

3.21123587054975

1.56943004865951

1.78252798168409

1.01650942697449

0.26405729485205

0.48520098822836

1.46392851541072

5.64663355823591
2.64851112412931

2.08759168533232

2.25282657017275

4.22587477652539

3.45669791836957

2.62758632426670

4.39615597177697

3.64602892941813

2.93401182631691

2.62400343650053

5.01991670872899

6.20113353292764

1.29465655297269

0.78267398716872

5.37930858706123

5.99270235506457

6.61611261988614

3.35382938330612

0.97392031673062

$-0.08242233876615$

4.87085252347890

7.27611591793915

2.85670826130116

1.77805897814896

3.39993284959163

3.03803607948845

4.51335384115846

3.55985411567149

4.40804344708818

5.29068652701152

1.64841464606243

1.40939358944446

7.67918215465982

7.78700922450613

8.18612757882508

6.20738406836434

7.03507909316376

6.12600434362119

6.48296786307767

6.61762807749463

5.84024062261315

7.37939463549279

6.16283713792005

1.00621047517321

0.83836074856654

0.08653664057792

1.27875598077076

$-1.43229725307930$

$-1.78254335408786$

$-2.20657676458029$

$-1.35091245665162$

3.76608146924294

3.97909903041869

3.69057231911298

2.77893393379241

4.87592429666435 
H 2.77993020874033

H 2.76896169968442

H 4.32414146319821

C 8.12724894986661

H 7.05106860449079

H 8.69712570682455

C 4.16728657717891

H 5.17820127583676

H 3.64462444086871

H 4.24497464547530

C 10.40147074954661

H 9.40847963383627

H 10.38639773920298

H 10.58844429695553

C 5.53944579962751

H 6.25410136354407

H 4.57109039955209

C 6.07016181499112

H 6.73274175040268

H 5.25518974622265

C 12.83882199126878

H 13.11323864890433

H 12.74524611408542

H 13.67145942445891

C 3.15919648247560

H 4.16817229345157

H 2.62726339054537

H 2.62779647533957

C 5.45505789590869

H 5.42951789893222

H 4.55756219793800

C 8.78567621157686

H 9.88602324469029

H 8.30482654251281

C 8.58489136433858

H 8.01685920515605

H 9.65707611719140

C 1.77905440809770

H 1.75487369387884

H 1.18054811148310

H 1.27912045792559

C 8.36271871925117

H 8.94687280479169

H 7.29308568762565

9_t

V 7.49418379352451

Cl 6.86611591774995

O 8.44712293115285

O 6.60666391664989

O 9.17553891538972

N 9.31761190599147

O 5.98032008182590

N 9.41245727977028

N 8.18609666434258

C 5.91558754942798
4.69894511458507

6.45902211756921

5.59404952469996

6.41462371618050

6.22181102585014

5.52562739988402

7.24139948319145

7.18656879522691

8.09262217927770

7.44922515482786

5.40729525353454

5.39553664227411

6.16845392856638

4.42216680617138

2.16554725719184

1.44105008448031

1.65306267663089

2.72810892730293

2.04285211301247

3.05381979279031

5.88347844830220

4.95325263596597

6.68168568968763

6.15917504136685

0.60218457416532

0.17286711472801

$-0.01688581404045$

0.51279574648702

3.42149564531043

3.19749280178164

4.01606067311751

7.95561037068171

7.93454743521257

8.25898548729433

7.72149932557971

7.94648523899302

7.67164374255014

2.57431920709303

3.61985695542916

2.51224232799462

1.95066263396169

8.74567998903001

9.67244740478743

9.01636407685428

5.31368049096934

7.12646348567669 6.64116600860764 3.95673462805983 4.97360495479526 3.16396814462300 5.28073052957290 2.23623341585342 2.20566005317473 3.33563968896265
5.07256923587685

5.38982317070869

5.31483836601041

1.97164835286026

1.81141372175601

1.65164384879689

3.10476936395972

3.53887985955443

3.57846775941806

2.02139118325680

8.30730367352794

7.83266174756812

9.10901115686352

8.77229187132992

7.20732188724572

7.63984838963756

7.07780412762688

5.88821813418473

5.33279468742714

5.21689149901190

8.03462576830920

8.56430373806728

8.79224397088447

7.36233724129770

0.37645777152642

0.50941411447665

$-0.36972943817117$

1.34072510498595

8.08932040386104

9.16926772717880

7.83619979489782

3.67570633972973

3.80206423675565

4.62064620026451

1.32126497774718

0.40263675303971

1.05566571299833

$-0.29977250623127$

$-0.65558131930245$

0.62665751044738

$-1.06235706623676$

2.44551633368521

2.31526312513743

2.52119045517567

4.81808490808056

6.18623484450566

3.47314423325273

6.16978498039713

5.63427436547653

3.49120205438352

3.68231342519265

2.52875916400037

1.99127647904207

2.28170757287223 


\begin{tabular}{|c|c|c|c|}
\hline & 10.012120 & 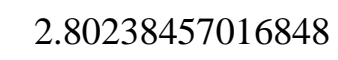 & \\
\hline & 11.70771786924318 & 3483481083404 & \\
\hline & 7.30927150586540 & 3.08402080892662 & 0 \\
\hline & 10.45096546542338 & 3.4450580529718 & 59 \\
\hline & 8.06736079722815 & 2877614363 & 3.60377956376755 \\
\hline & 5.31381457488418 & 4.5251 & \\
\hline & 3.98292177567042 & & 2.44410745225736 \\
\hline & 12.78819619478967 & & 063 \\
\hline & 49592 & 4157 & 29830756112 \\
\hline & 5.13517270822402 & 2.49241002843560 & 1.45768822580864 \\
\hline & & 1.55045940351746 & \\
\hline & & & \\
\hline & & & \\
\hline & & & \\
\hline & & 6.2061 & \\
\hline & & & \\
\hline & & 1.8 & \\
\hline & & & \\
\hline & & & \\
\hline & & 6.41 & \\
\hline & & & \\
\hline & & & \\
\hline & & 5.6 & \\
\hline & & & \\
\hline & & & \\
\hline & & & \\
\hline & & & \\
\hline & & 4.0 & \\
\hline & & & \\
\hline & & & \\
\hline & & & \\
\hline $\mathrm{H}$ & & & \\
\hline $\mathrm{C}$ & & & \\
\hline $\mathrm{H}$ & & & \\
\hline $\mathrm{H}$ & & & \\
\hline $\mathrm{H}$ & & & \\
\hline $\mathrm{C}$ & & & \\
\hline $\mathrm{H}$ & & & \\
\hline 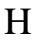 & & & 659 \\
\hline 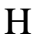 & & & \\
\hline t & & & \\
\hline 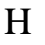 & & & \\
\hline 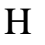 & & & \\
\hline 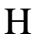 & & & \\
\hline 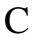 & & & -1.1 \\
\hline $\mathrm{H}$ & & & 999 \\
\hline 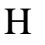 & & 0.9 & 3971 \\
\hline $\mathrm{H}$ & & 1.19 & -0.9 \\
\hline $\mathrm{C}$ & 14. & 0256 & 38541 \\
\hline $\mathrm{H}$ & & 62648 & 5683 \\
\hline $\mathrm{H}$ & & 5644 & 458344 \\
\hline $\mathrm{H}$ & 560702 & 1685968039 & 7816408470 \\
\hline $\mathrm{C}$ & & 6.211 & 989778 \\
\hline $\mathrm{H}$ & & & 62593 \\
\hline $\mathrm{H}$ & & & \\
\hline$U_{0}$ & 4.22828109255076 & 6.22315612842535 & 4.95654398568371 \\
\hline
\end{tabular}


C 8.17611211892735

H 7.12957131187359

H 8.28599204478691

C 4.22347695463855

H 5.22005419110718

H 3.74868262810167

H 4.33827213772416

C 10.19328429908461

H 9.21432935445470

H 10.11034244034705

H 10.43585917198322

C 6.64483675104399

H 7.21129950368649

H 6.68117460108089

C 7.21893155669964

H 8.31171722093923

H 6.94045458920121

C 12.60794640291079

H 12.92145175785803

H 12.46103338101434

H 13.43694959138634

C 2.87292637180211

H 3.85960667486992

H 2.30185471978292

H 2.34684922226733

C 5.21582689971213

H 4.74475034942989

H 4.57251522832432

C 9.30975571801292

H 10.30954934670891

H 8.87223855421804

C 9.18224721921614

H 8.82826206227949

H 10.14839051722329

C 1.59625592575600

H 1.62685828767509

H 1.00436595346389

H 1.05447600151798

C 9.33000244947452

H 10.25124499247674

H 8.46673238432381
6.69648953894602

7.02217429741319

5.67660386270722

7.40532054247692

7.35746662295349

8.35480462581558

7.42104439605487

4.80033408531910

4.81806605848082

5.39469993631755

3.75879612461783

2.39682990207118

2.94850078428605

1.32213127722286

2.70036521070063

2.83758219482779

1.93308014297380

5.43361583507369

4.42795216879053

6.05305963662335

5.88136813362627

0.47106783033345

0.02256598266169

$-0.24812440684649$

0.59387672098704

2.94898803179696

3.16302379282383

2.22813359591602

7.73613125955772

7.31933598185734

8.16279413716946

7.70772740210063

8.19167452229877

7.21516261978319

2.35806096270146

3.32102787319586

2.50021622436363

1.63487183307336

8.67979117479753

9.28506441842438

9.36937212454397
2.05100687012626

1.90386410138981

1.64405810846057

2.49224698537457

2.95881991189591

2.80095803258934

1.39285683369624

8.45184785593074

7.94656174227914

9.38041538221526

8.73216102599509

7.92359342523766

8.69652479625303

8.16979089300699

6.54408411690051

6.52835713753096

5.79338918012622

8.34819230815084

8.68261174424034

9.25075864157357

7.77029943074865

0.95218621099743

1.16523659353218

0.33561724938070

1.91569021579623

7.80029449545841

8.77444983651945

7.26236262726936

3.88698236588118

4.11473951664153

4.80527708967758

1.50609913813030

0.58008102381188

1.28926721409600

$-0.07993327271190$

$-0.62057506918139$

0.84187795722208

$-0.71562105130045$

2.68777169479459

2.64524105632872

2.72915954674709 


\section{EPR Spectroscopy}

Simulated EPR-Spectra for complexes $\left[\mathrm{Co}\left(\mathbf{C p}^{*}\right)_{2}\right][1]$ and $\left[\mathrm{Co}\left(\mathbf{C p}^{*}\right)_{2}\right][2]$ were obtained using the garlic function (non linear least squares fitting) of the EasySpin package in MATLAB ${ }^{\circledR}{ }^{6}$ The lowest RMSD values were found at $\mathrm{g}_{\text {iso }}=1.9715, \mathrm{a}=[274.1915 \mathrm{MHz}, 268.8744 \mathrm{MHz}, 258.604 \mathrm{MHz}]$, Lorentzian linewidth $=1.344 \mathrm{MHz}$ and $28.9 \mathrm{~ns}$ correlation time for $\left[\mathrm{Co}\left(\mathrm{Cp}^{*}\right)_{2}\right][1]$ and at $\mathrm{g}_{\text {iso }}=1.9666, \mathrm{a}=[256.8$ $\mathrm{MHz} 267.7 \mathrm{MHz}, 261.9 \mathrm{MHz}$, Lorentzian linewidth $=1.616 \mathrm{MHz}$ and $31.0 \mathrm{~ns}$ correlation time for $\left[\mathrm{Co}(\mathrm{Cp} *)_{2}\right][2]$.

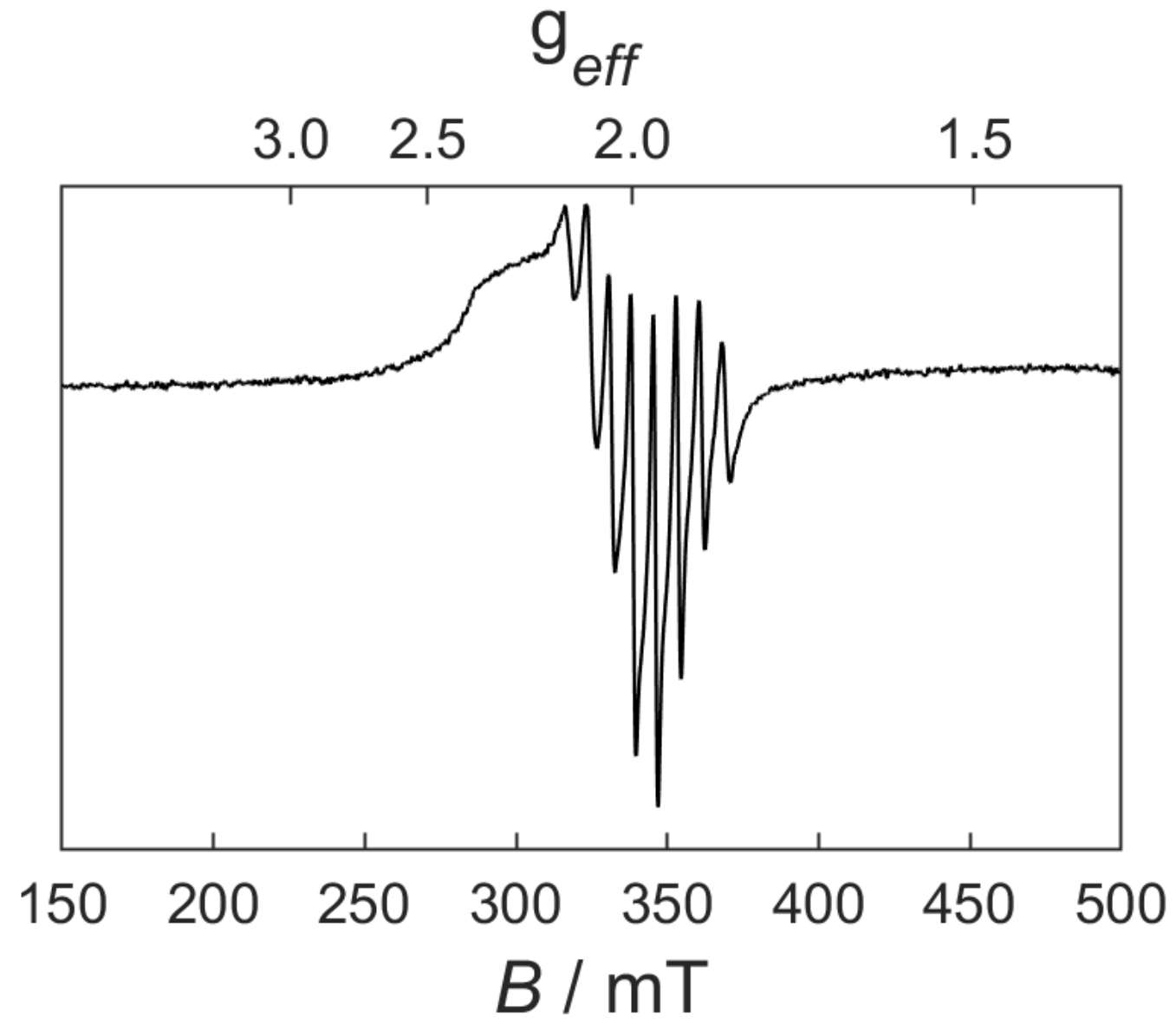

Figure S 90: $\quad X$-band EPR spectrum of a $5 \mathrm{mM}$ solution of 6 in toluene at $300 \mathrm{~K}$. The eight-line signal observed at $g_{\text {iso }}=1.9681$ results most likely from a V(IV) impurity. 


\section{Crystallographic details}

Table S 2: Crystallographic details

\begin{tabular}{|c|c|c|c|c|c|c|c|c|c|}
\hline & $1^{*}$ & 2 & $\left.\left[\mathrm{Co}(\mathrm{Cp})_{2}\right)_{2}\right][1]^{* *}$ & {$\left[\mathrm{Co}\left(\mathrm{Cp}^{*}\right)_{2}\right][2]^{* *}$} & $3^{* *}$ & 5 & $6 * * *$ & 8 & $10^{* *}$ \\
\hline Chemical formula & $\begin{array}{l}\mathrm{C}_{31} \mathrm{H}_{43} \mathrm{~N}_{3} \mathrm{O}_{3} \mathrm{Cl}_{1} \mathrm{~V}_{1} \\
\mathrm{C}_{7} \mathrm{H}_{8}\end{array}$ & $\mathrm{C}_{35} \mathrm{H}_{44} \mathrm{~N}_{2} \mathrm{O}_{3} \mathrm{Cl}_{1} \mathrm{~V}_{1}$ & $\begin{array}{l}\mathrm{C}_{20} \mathrm{H}_{30} \mathrm{Co}_{1} \\
\mathrm{C}_{31} \mathrm{H}_{43} \mathrm{~N}_{3} \mathrm{O}_{3} \mathrm{Cl}_{1} \mathrm{~V}_{1}\end{array}$ & $\begin{array}{l}\mathrm{C}_{20} \mathrm{H}_{30} \mathrm{Co}_{1} \\
\mathrm{C}_{35} \mathrm{H}_{44} \mathrm{~N}_{2} \mathrm{O}_{2} \mathrm{Cl}_{1} \mathrm{~V}_{1}\end{array}$ & $\mathrm{C}_{40} \mathrm{H}_{54} \mathrm{~N}_{3} \mathrm{O}_{4} \mathrm{~V}_{1}$ & $\mathrm{C}_{45} \mathrm{H}_{57} \mathrm{~N}_{4} \mathrm{O}_{2} \mathrm{~V}_{1}$ & $2\left(\mathrm{C}_{39} \mathrm{H}_{59} \mathrm{~N}_{3} \mathrm{O}_{4} \mathrm{Cl}_{1} \mathrm{~V}_{1}\right)$ & $\begin{array}{l}\mathrm{C}_{37} \mathrm{H}_{47} \mathrm{~N}_{4} \mathrm{O}_{2} \mathrm{~F}_{1} \mathrm{Cl}_{1} \mathrm{~V}_{1} \\
\mathrm{C}_{4} \mathrm{H}_{10} \mathrm{O}_{1}\end{array}$ & $2\left(\mathrm{C}_{34} \mathrm{H}_{52} \mathrm{~N}_{4} \mathrm{O}_{2} \mathrm{Si}_{1} \mathrm{Cl}_{1} \mathrm{~V}_{1}\right)$ \\
\hline$M_{\mathrm{r}}$ & 684.20 & 627.11 & 921.44 & 956.48 & 691.80 & 752.88 & 1440.56 & 759.29 & 1326.55 \\
\hline Crystal system & Monoclinic & Orthorhombic & Monoclinic & Orthorhombic & Monoclinic & Triclinic & Triclinic & Monoclinic & Monoclinic, \\
\hline Space group & $P 2_{1} / n$ & $\mathrm{Pbca}$ & $P 2_{1} / n$ & Pbca & $P 2_{1} / c$ & $P-1$ & P-1 & $P 2_{1} / n$ & $P 2_{1} / n$ \\
\hline a ( $(\AA)$ & $10.707(2)$ & $13.701(1)$ & $12.5450(5)$ & $19.5719(6)$ & $29.460(4)$ & $13.1076(8)$ & $15.404(3)$ & $16.8943(10)$ & $18.8519(15)$ \\
\hline b $(\AA ̊)$ & $22.859(4)$ & $16.588(1)$ & $19.1392(8)$ & $23.6798(8)$ & $14.896(2)$ & $13.5852(9)$ & $16.355(3)$ & $14.5056(9)$ & $10.7090(10)$ \\
\hline$c(\AA)^{\prime}$ & $15.010(3)$ & $29.021(3)$ & $22.4206(9)$ & $24.1624(7)$ & $20.399(2)$ & $14.0621(9)$ & $17.174(3)$ & $17.7402(12)$ & $40.126(3)$ \\
\hline$\alpha\left(^{\circ}\right)$ & 90 & 90 & 90 & 90 & 90 & $118.469(3)$ & $90.146(7)$ & 90 & 90 \\
\hline$\beta\left(^{\circ}\right)$ & $92.85(1)$ & 90 & $103.168(2)$ & 90 & $96.179(4)$ & $94.955(3)$ & $96.074(8)$ & $107.698(2)$ & $99.247(2)$ \\
\hline$y\left({ }^{\circ}\right)$ & 90 & 90 & 90 & 90 & 90 & 104.110() & $97.907(8)$ & 90 & 90 \\
\hline$V\left(\AA^{3}\right)$ & $3669(1)$ & $6595.8(9)$ & $5241.7(4)$ & $11198.3(6)$ & $8900(2)$ & 2073.7(2) & $4261.0(14)$ & $4141.7(5)$ & $7995.5(11)$ \\
\hline z & 4 & 8 & 4 & 8 & 8 & 2 & 2 & 4 & 4 \\
\hline Densitiy $\left(\mathrm{g} \mathrm{cm}^{-3}\right)$ & 1.239 & 1.263 & 1.168 & 1.135 & 1.033 & 1.206 & 1.123 & 1.218 & 1.102 \\
\hline$F(000)$ & 1456 & 2656 & 1964 & 4072 & 2960 & 804 & 1544 & 1616 & 2832 \\
\hline Radiation Type & MoK $\alpha$ & MoKa & MoK $\alpha$ & MoK $\alpha$ & MoKa & MoK $\alpha$ & MoKa & MoKa & MoKa \\
\hline$\mu\left(\mathrm{mm}^{-1}\right)$ & 0.382 & 0.418 & 0.586 & 0.550 & 0.259 & 0.282 & 0.333 & 0.349 & 0.376 \\
\hline Crystal size & $0.09 \times 0.08 \times 0.06$ & $0.04 \times 0.03 \times 0.004$ & $0.15 \times 0.13 \times 0.05$ & $0.20 \times 0.15 \times 0.01$ & $0.11 \times 0.10 \times 0.02$ & $0.08 \times 0.07 \times 0.06$ & $0.25 \times 0.15 \times 0.03$ & $0.45 \times 0.10 \times 0.07$ & $0.45 \times 0.25 \times 0.10$ \\
\hline Meas. Refl. & 101324 & 27019 & 82542 & 109455 & 71804 & 142685 & & 56058 & 78415 \\
\hline Indep. Refl. & 6470 & 5999 & 9202 & 9863 & 7840 & 9522 & & 7658 & 14841 \\
\hline Obsvd. $[I>2 \sigma(I)]$ & 5363 & 4402 & 8231 & 6483 & 4512 & 7698 & & 303 & 9958 \\
\hline $\mathrm{R}_{\text {int }}$ & 0.0909 & 0.1015 & 0.0424 & 0.1203 & 0.1753 & 0.1031 & & 0.1031 & 0.0957 \\
\hline$R\left[F^{2}>2 \sigma\left(F^{2}\right)\right]$ & 0.0632 & 0.0613 & 0.0426 & 0.1028 & 0.0725 & 0.0443 & & 0.0452 & 0.0741 \\
\hline $\mathrm{WR}\left(\mathrm{F}^{2}\right)$ & 0.1774 & 0.1327 & 0.1275 & 0.3127 & 0.1673 & 0.1153 & & 0.1026 & 0.1769 \\
\hline$S$ & 1.092 & 1.073 & 1.027 & 1.18 & 1.016 & 1.055 & & 1.019 & 1.026 \\
\hline$\Delta \rho_{\max }$ & 2.453 & 0.385 & 1.484 & 0.7446 & 0.329 & 0.820 & & 0.281 & 0.751 \\
\hline$\Delta \rho_{\min }$ & -0.844 & -0.461 & -0.720 & -0.452 & -0.428 & -0.322 & & -0.345 & -0.743 \\
\hline CCDC & 2079467 & 2079465 & 2079472 & 2079471 & 2079474 & 2079469 & 2096422 & 2079473 & 2080686 \\
\hline
\end{tabular}

* Due to moderate quality of the crystals a minor positive electron density remains close to the vanadium center and cannot be refined. This most likely resulted as an artefact from the measurement and is typical for heavy atom containing structures. ${ }^{* *}$ Due to heavily disordered solvent molecules, the SQUEEZE algorithm was applied. For the cationic complexes $\left[\mathrm{Co}\left(\mathbf{C p}^{*}\right)_{2}\right][1]$ and $\left[\mathbf{C o}(\mathbf{C p} *)_{2}\right][2]$ disordered dichloromethane was squeezed, for the neutral complexes $\mathbf{3}$ and $\mathbf{1 0}$ heavily disordered diethyl ether was removed from the lattice. ${ }^{* * *}$ Due to the high sensitivity of this compound (less than 30 seconds in air) and the propensity of the compound to only form small and disordered crystals, these values are only given for orientation. 
Table S 3: Selected Bond lengths and angles

\begin{tabular}{|c|c|c|c|c|c|c|c|c|}
\hline & 1 & 2 & {$\left[\mathrm{Co}\left(\mathrm{Cp}^{*}\right)_{2}\right][1]$} & {$\left[\mathrm{Co}\left(\mathrm{Cp}^{*}\right)_{2}\right][2]$} & 3 & 5 & 8 & 10 \\
\hline $\mathrm{V} 1-\mathrm{C} 1$ & $2.055(3)$ & $2.131(3)$ & $2.070(3)$ & $2.153(7)$ & $2.067(3)$ & $2.0830(18)$ & $2.048(2)$ & $2.048(4)$ \\
\hline $\mathrm{V} 1-01$ & $1.823(2)$ & $1.830(2)$ & $1.935(2)$ & $1.916(6)$ & $1.853(2)$ & $1.9037(13)$ & $1.835(2)$ & $1.845(3)$ \\
\hline $\mathrm{V} 1-02$ & $1.843(2)$ & $1.827(2)$ & $1.945(2)$ & $1.901(6)$ & $1.901(3)$ & $1.9050(13)$ & $1.858(2)$ & $1.864(3)$ \\
\hline V1 - 010/N40 & $1.583(3)$ & $1.585(2)$ & $1.603(2)$ & $1.618(6)$ & $1.589(3)$ & $1.5865(13)$ & $1.644(2)$ & $1.621(4)$ \\
\hline $\mathrm{V} 1-\mathrm{X}^{*}$ & $2.273(1)$ & $2.275(1)$ & $2.366(1)$ & $2.331(3)$ & $1.811(2)$ & $1.9161(15)$ & $2.3045(7)$ & $2.3184(12)$ \\
\hline $\mathrm{N} 1-\mathrm{C} 8$ & - & $1.426(1)$ & - & $1.400(10)$ & - & - & - & - \\
\hline $\mathrm{C} 8-\mathrm{C} 13$ & - & $1.399(5)$ & - & $1.410(11)$ & - & - & - & - \\
\hline $\mathrm{C} 13-\mathrm{O} 1$ & - & $1.351(4)$ & - & $1.294(10)$ & - & - & - & - \\
\hline $\mathrm{C} 2-\mathrm{C} 5$ & $1.446(5)$ & - & $1.438(4)$ & - & $1.454(5)$ & $1.449(2)$ & $1.453(3)$ & $1.460(5)$ \\
\hline $\mathrm{C} 5-\mathrm{C} 10$ & $1.405(5)$ & - & $1.415(4)$ & - & $1.418(5)$ & $1.413(3)$ & $1.412(3)$ & $1.409(5)$ \\
\hline $\mathrm{C} 10-\mathrm{O} 1$ & $1.347(4)$ & - & $1.322(3)$ & - & $1.340(4)$ & $1.337(2)$ & $1.343(3)$ & $1.337(4)$ \\
\hline $\mathrm{N} 1-\mathrm{C} 11$ & $1.435(4)$ & - & $1.427(3)$ & - & $1.430(5)$ & $1.425(2)$ & $1.433(3)$ & $1.426(5)$ \\
\hline $\mathrm{C} 11-\mathrm{C} 16$ & $1.389(5)$ & - & $1.402(3)$ & - & $1.400(5)$ & $1.402(2)$ & $1.392(3)$ & $1.399(5)$ \\
\hline $\mathrm{C} 16-\mathrm{O} 2$ & $1.345(4)$ & - & $1.322(3)$ & - & $1.341(4)$ & $1.330(2)$ & $1.342(3)$ & $1.345(5)$ \\
\hline $01-\mathrm{V} 1-02$ & $146.4(1)$ & $142.5(1)$ & $145.2(1)$ & $146.1(3)$ & $146.81(12)$ & $148.01(6)$ & $145.98(8)$ & $144.69(12)$ \\
\hline $\mathrm{C} 1-\mathrm{V} 1-\mathrm{X}^{*}$ & 149.6(1) & 154.2(9) & $143.4(1)$ & 139.1(2) & $151.07(15)$ & 147.31(8) & $152.21(7)$ & $150.57(1)$ \\
\hline O10/N40-V1 - C1 & $99.6(2)$ & $98.1(1)$ & $107.6(1)$ & 109.1(3) & $100.05(13)$ & $104.23(7)$ & $94.81(9)$ & $94.86(17)$ \\
\hline 010/N40 - V1 - X* & $110.8(1)$ & 107.7(1) & 109.1(8) & $111.8(2)$ & $108.68(13)$ & $108.45(7)$ & $112.94(7)$ & $114.5(14)$ \\
\hline$\tau_{5}$ & 0.05 & 0.20 & 0.03 & 0.12 & 0.07 & 0.01 & 0.10 & 0.10 \\
\hline
\end{tabular}

${ }^{*} \mathrm{X}=\mathrm{Cl} 1, \mathrm{O} 40, \mathrm{~N} 10$ 


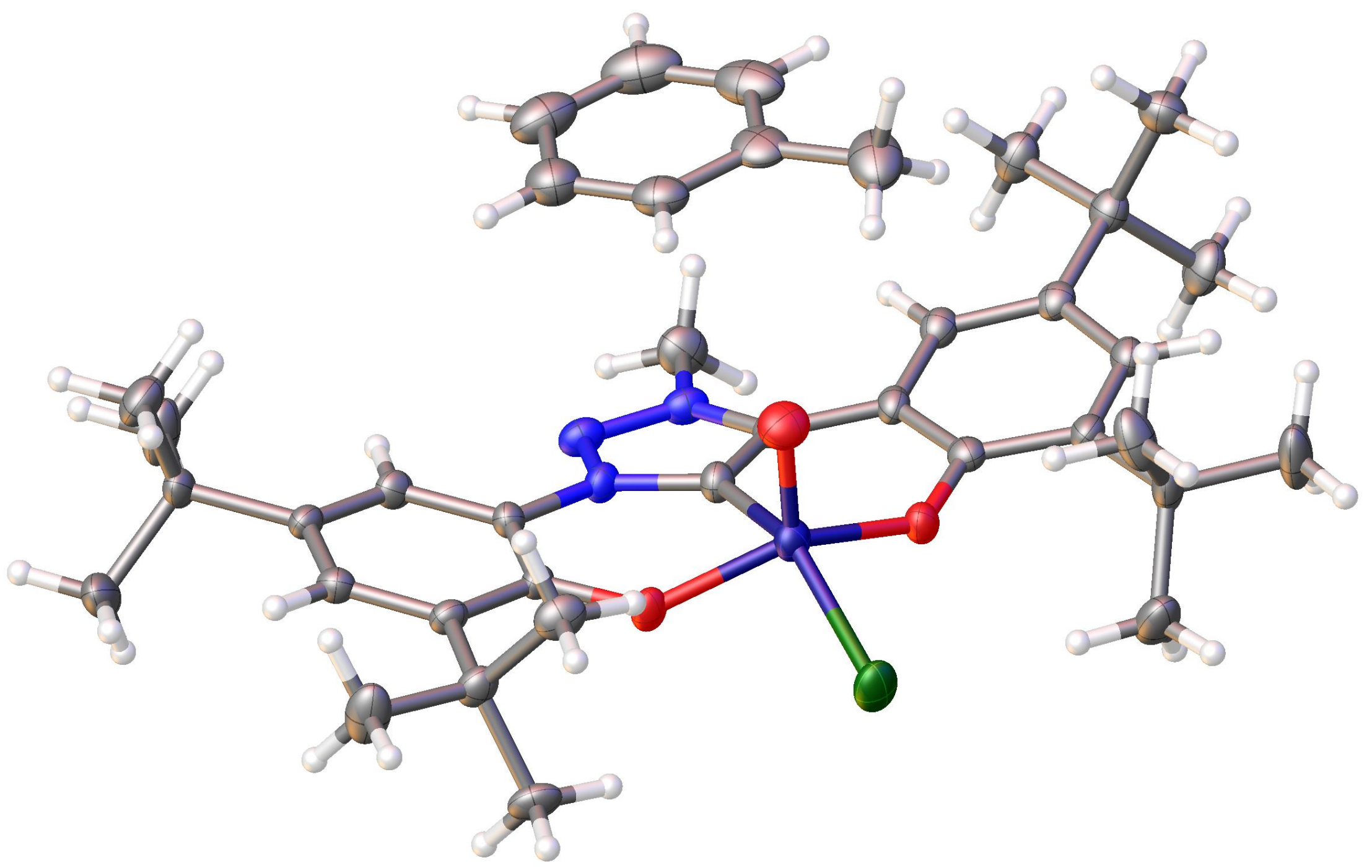

Figure S 91: Ellipsoid plot of 1 with all solvents in the asymmetric unit. Ellipsoids are shown at a probability level of $50 \%$. 


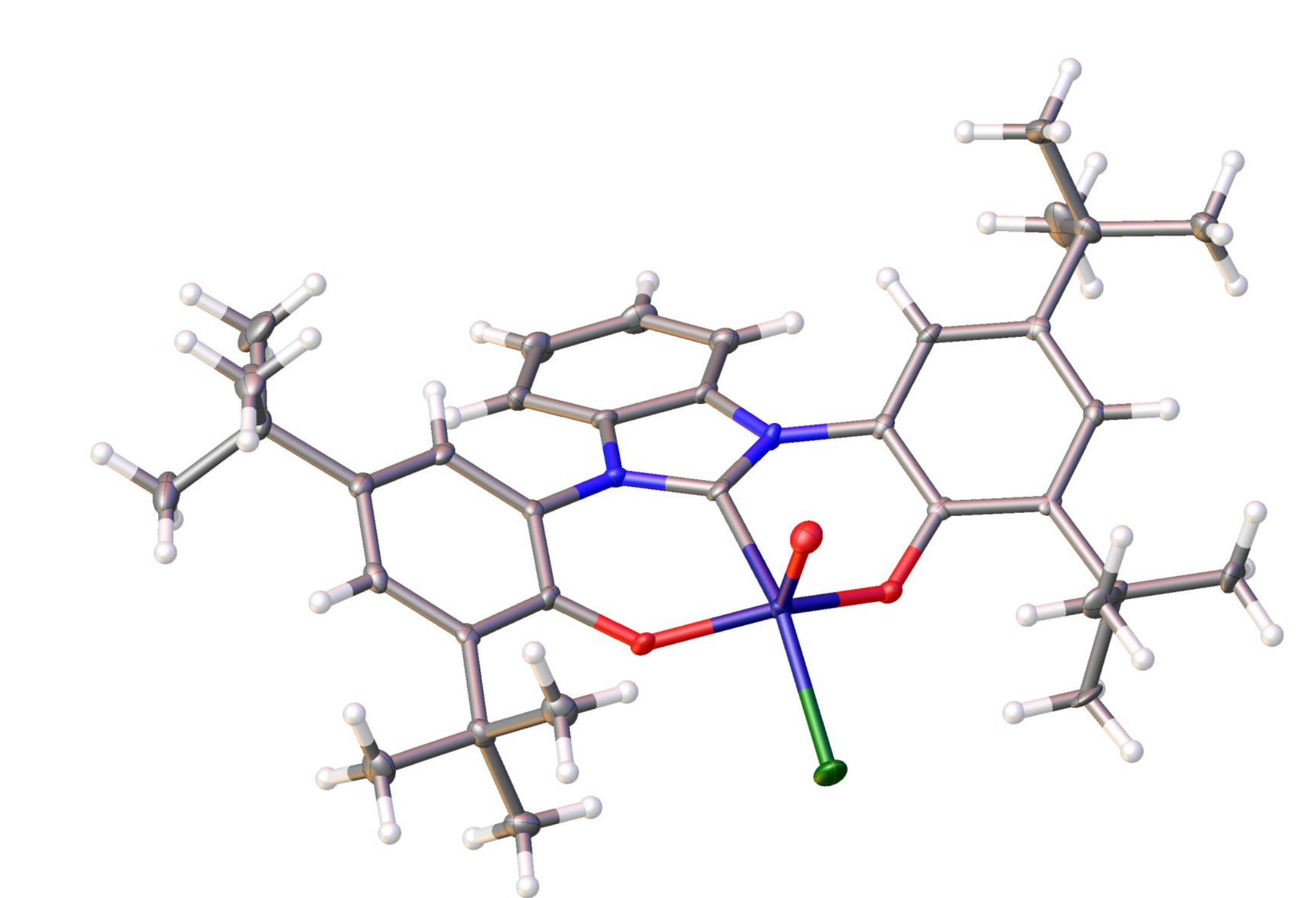




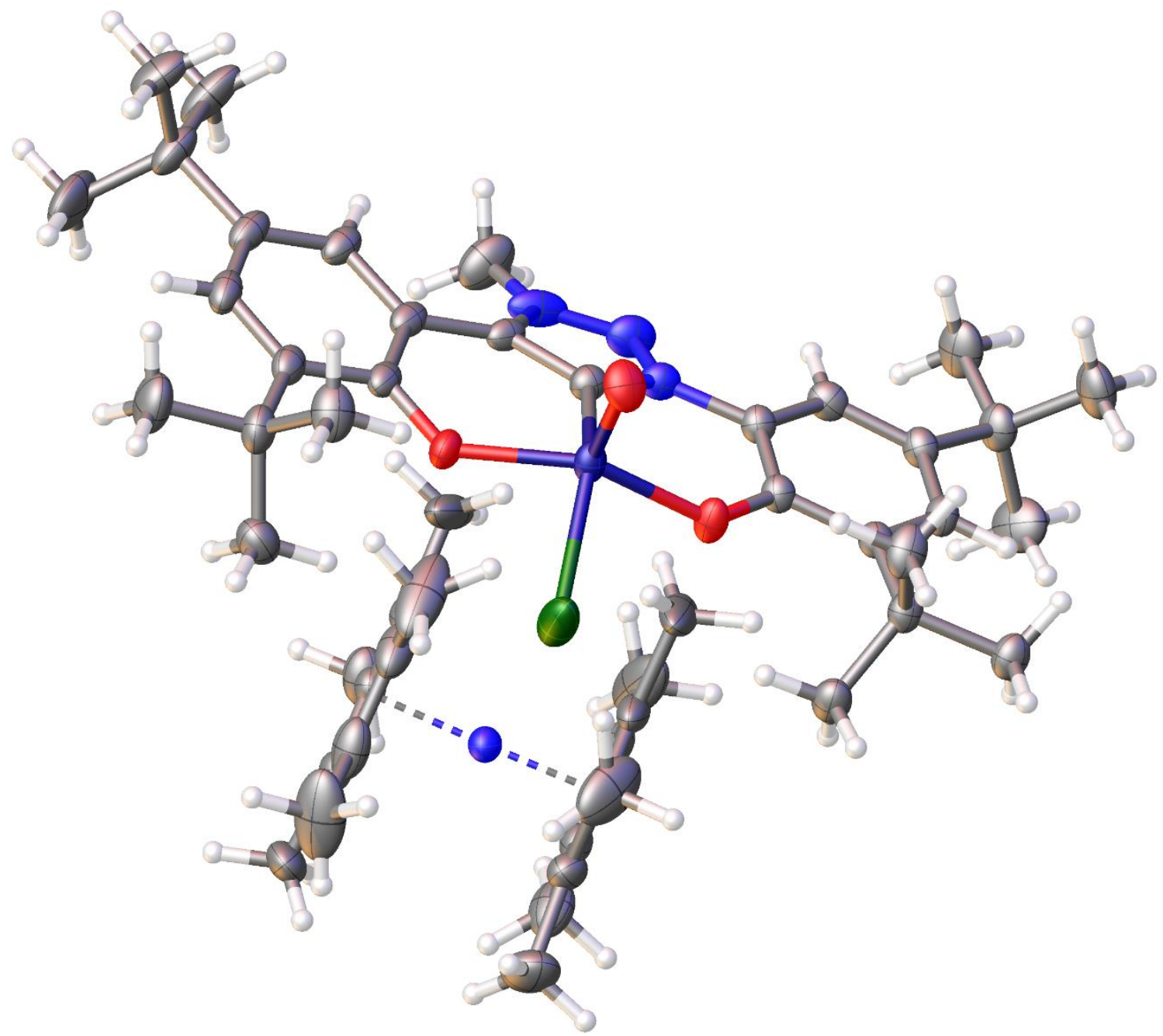

Figure S 93: Ellipsoid plot of $\left[\mathrm{Co}\left(\mathrm{Cp}^{*}\right)_{2}\right][1]$ including the decamethyl cobaltocenium counterion. Ellipsoids are shown at a probability level of $50 \%$. A heavily disordered molecule of dichloromethane could not be sufficiently modeled and was squeezed from the lattice. 


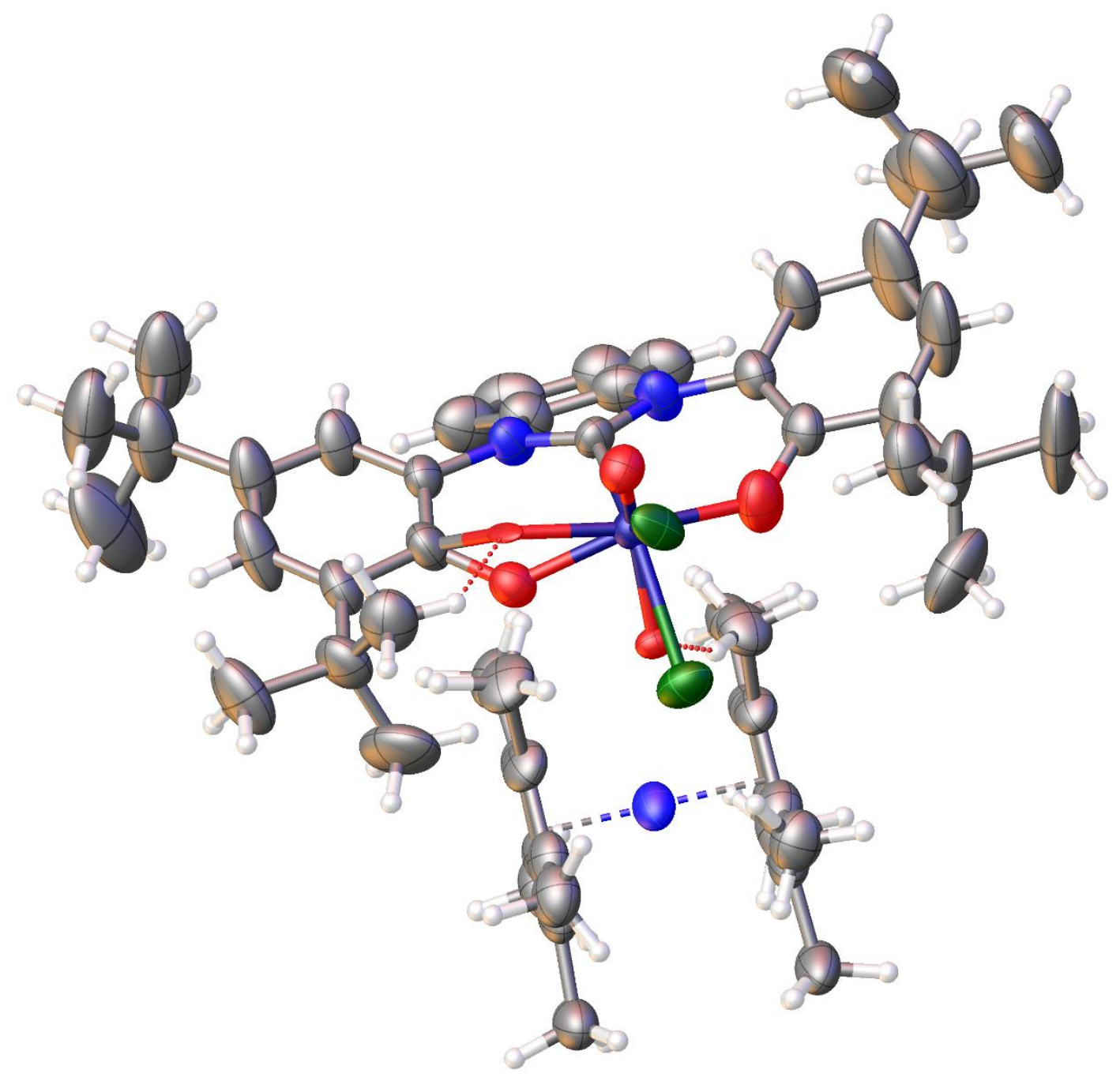

Figure S 94: Ellipsoid plot of [ $\left.\mathrm{Co}\left(\mathrm{Cp}^{*}\right)_{2}\right][2]$ including the decamethyl cobaltocenium counterion. Ellipsoids are shown at a probability level of $50 \%$. The full molecule disorder $\left(2^{\text {nd }}\right.$ part is less than $10 \%$ ) could not be completely resolved and was only refined for the central vanadium atom, the vanadium oxygen and one phenolate oxygen atom. A heavily disordered molecule of dichloromethane could not be sufficiently modeled and was squeezed from the lattice. 


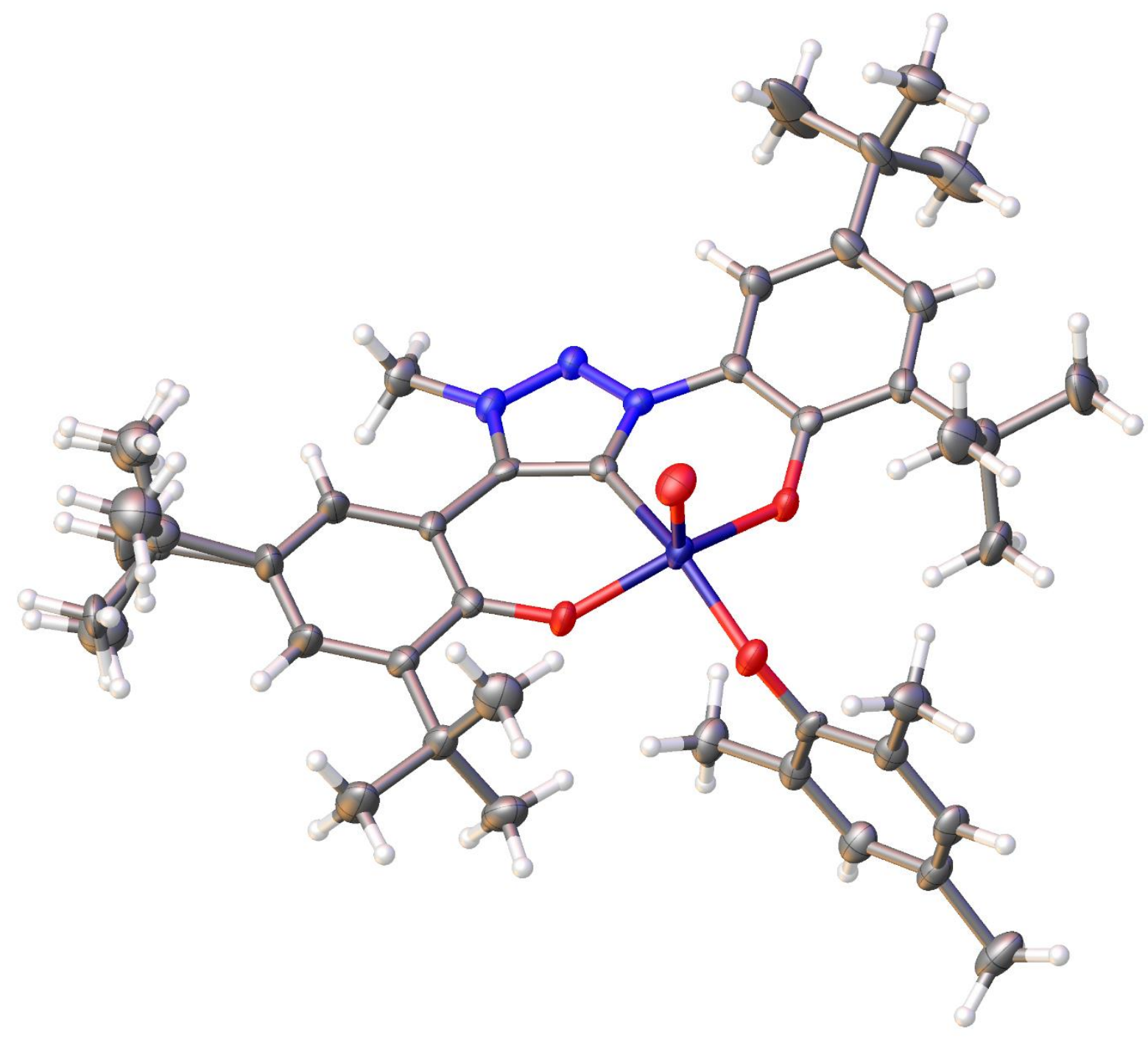

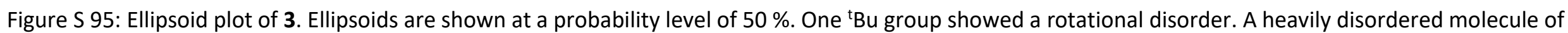
diethyl ether could not be sufficiently modeled and was squeezed from the lattice. 


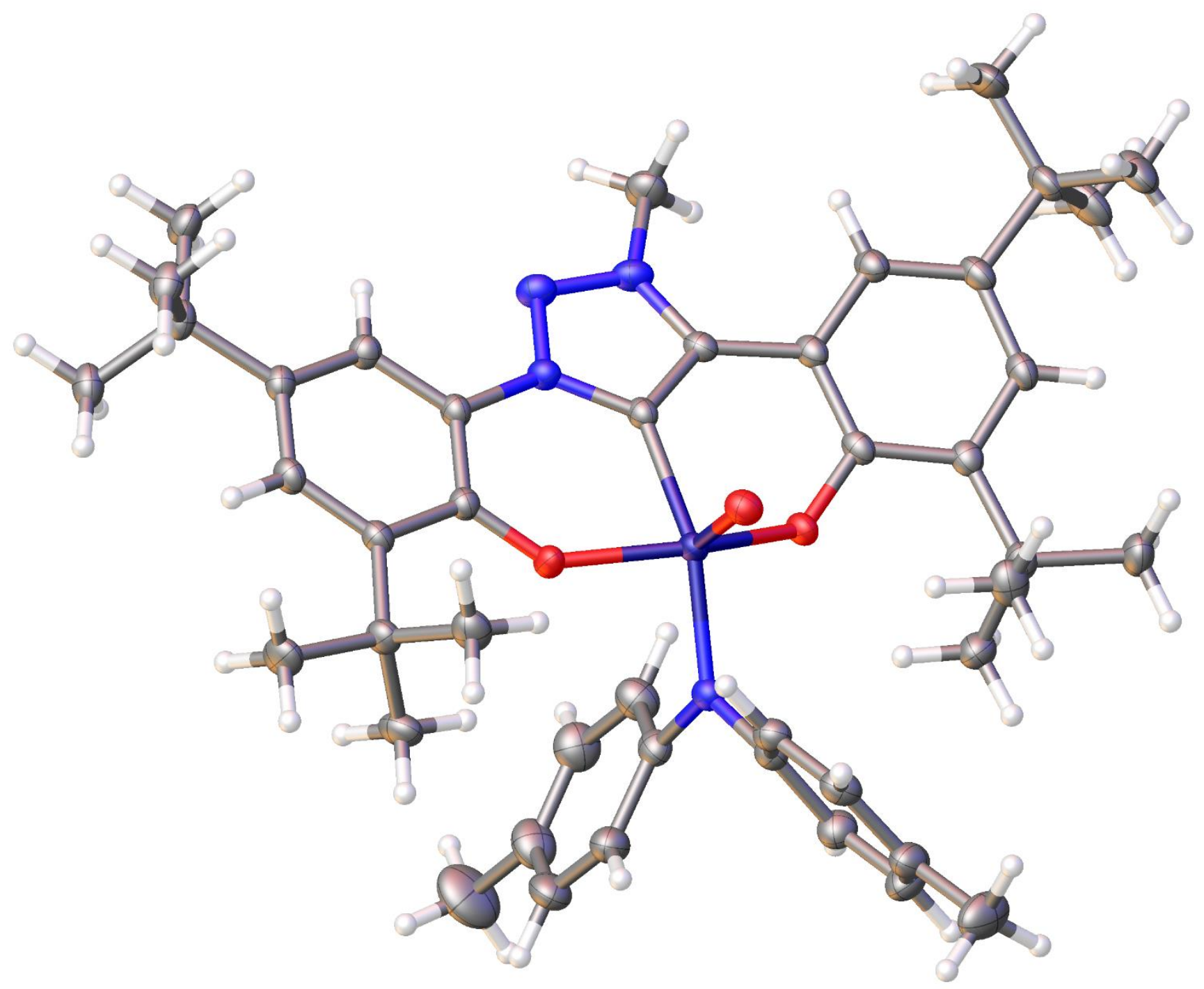

Figure S 96: Ellipsoid plot of 5. Ellipsoids are shown at a probability level of $50 \%$. 


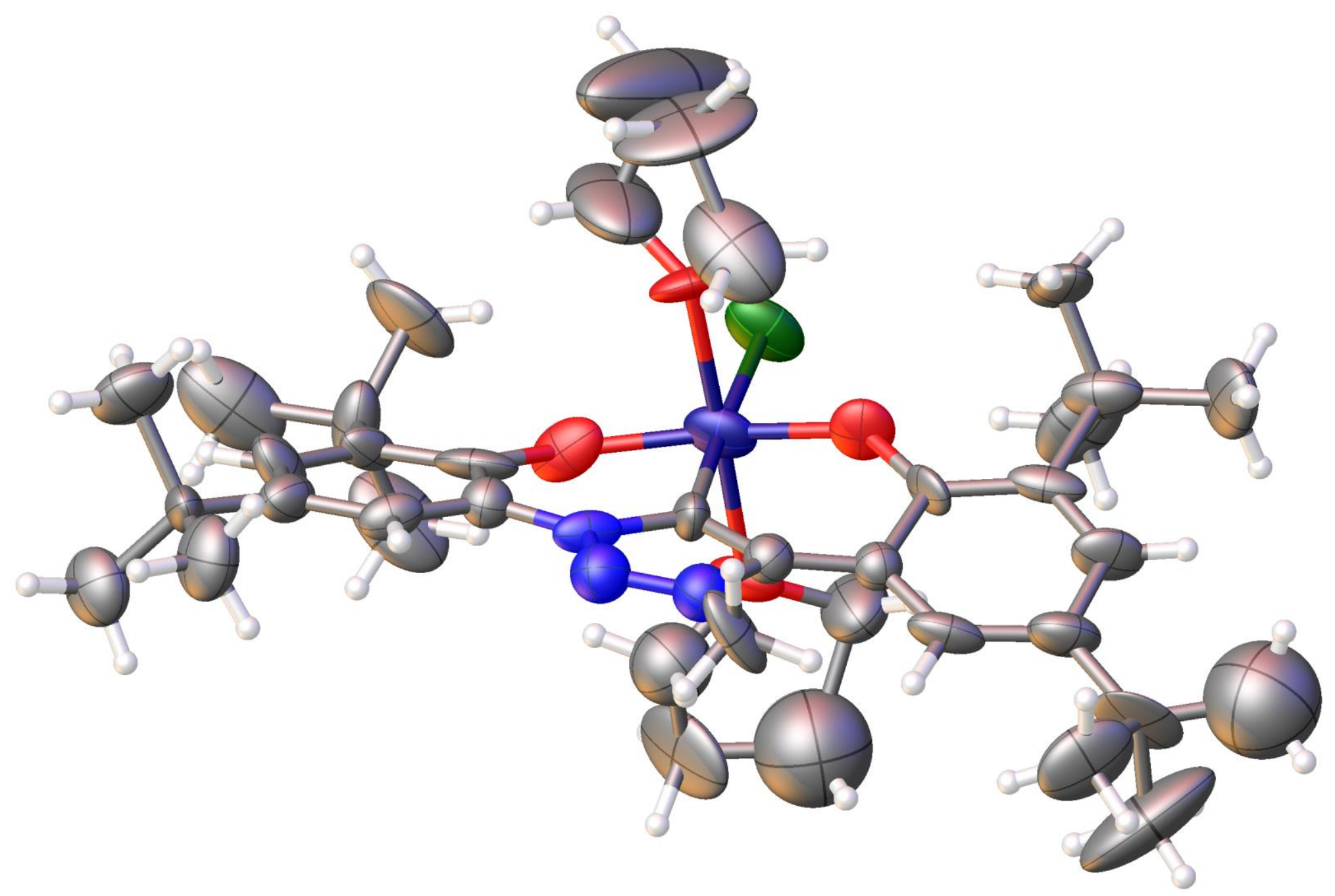

Figure S 97: Ellipsoid plot of 6 . Ellipsoids are shown at a probability level of $50 \%$. Due to the very low crystal quality, it was only possible to get sufficient data up to $35^{\circ}$ in $2 \theta$. The model as such cannot be considered reliable however it matches well with other analytical means suggesting that at least the connectivity and overall conformation of the molecule are correctly described. 


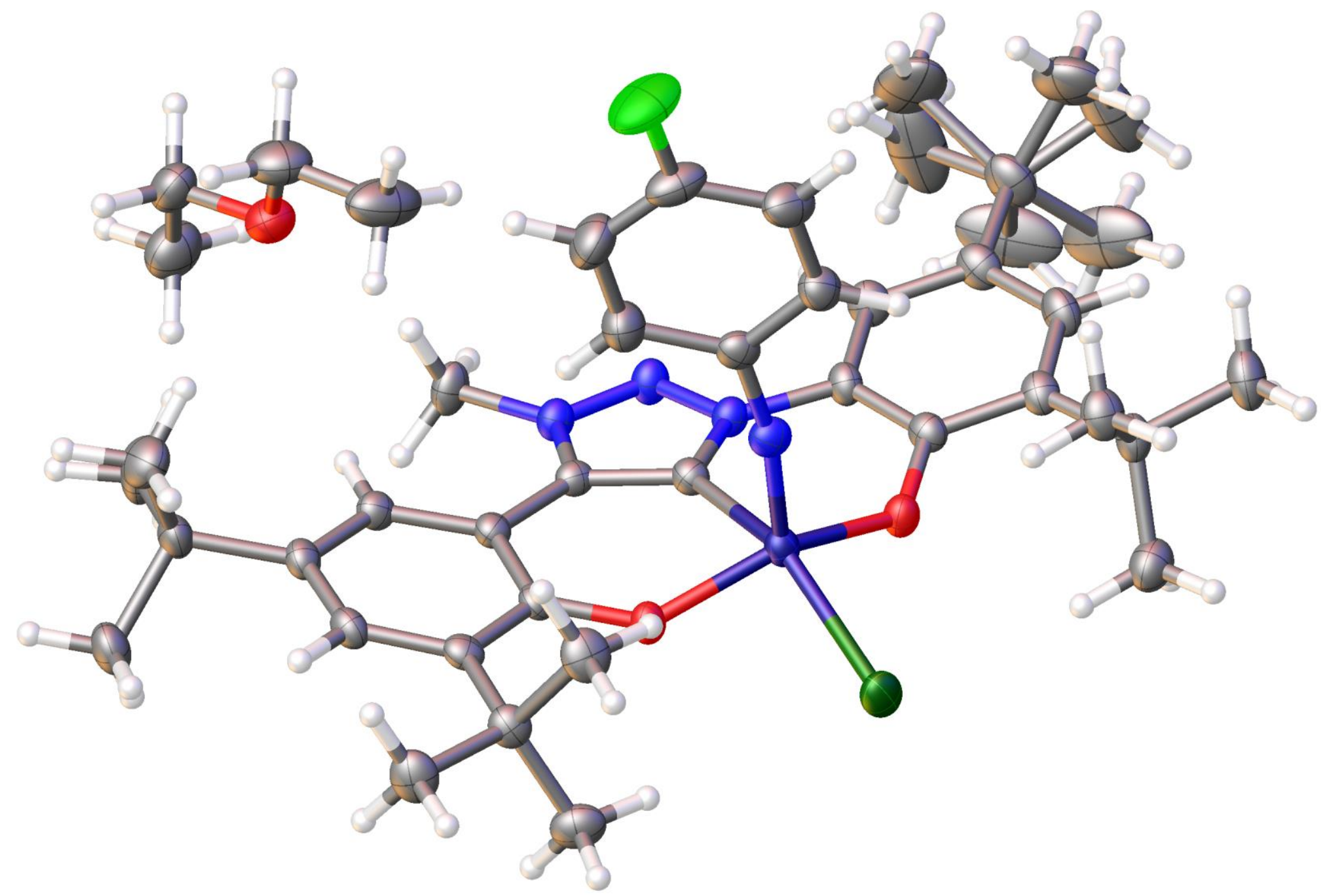

Figure S 98: Ellipsoid plot of 8 . Ellipsoids are shown at a probability level of $50 \%$. One of the ${ }^{\mathrm{t} B u}$ groups showed a rotational disorder. 


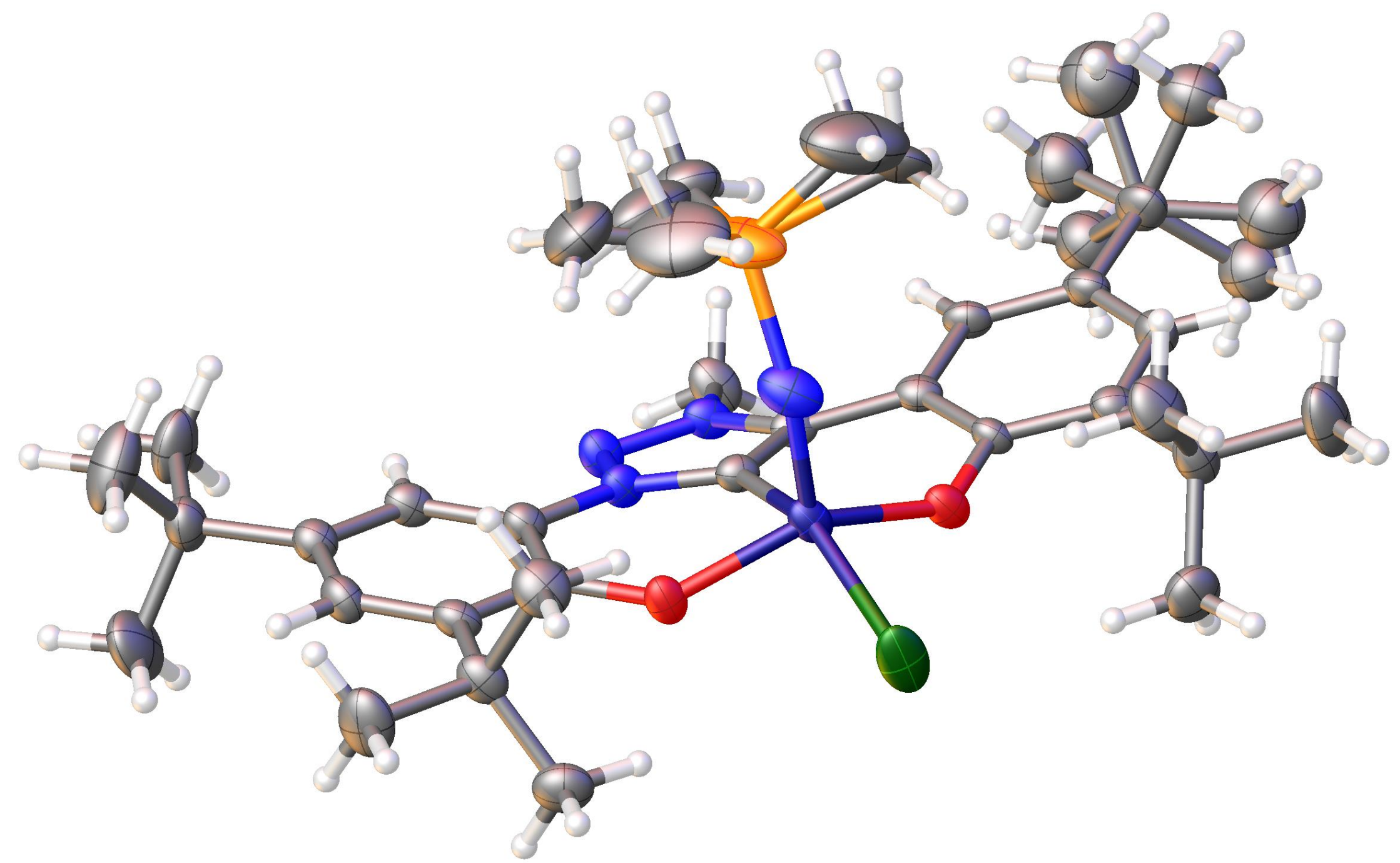

Figure S 99: Ellipsoid plot of 10. Ellipsoids are shown at a probability level of $50 \%$. The TMS group and one of the ${ }^{\mathrm{t} B u}$ groups showed a rotational disorder. A molecule of heavily disordered diethyl ether could not be sufficiently modeled and was therefore squeezed from the lattice. 


\section{Literature}

(1) a) F. Neese, Wiley Interdiscip. Rev.: Comput. Mol. Sci. 2018, 8, e1327; b) F. Neese, Wiley Interdiscip. Rev.: Comput. Mol. Sci. 2012, 2, 73-78.

(2) a) S. Grimme, S. Ehrlich, L. Goerigk, J. Comput. Chem. 2011, 32, 1456-1465; b) S. Grimme, J. Antony, S. Ehrlich, H. Krieg, J. Chem. Phys. 2010, 132, 154104; d) J. P. Perdew, Phys. Rev. B 1986, 33, 8822-8824; e) F. Weigend, R. Ahlrichs, Phys. Chem. Chem. Phys. 2005, 7, 32973305; g) J. P. Perdew, K. Burke, M. Ernzerhof, Phys. Rev. Lett. 1996, 77, 3865-3868.

(3) D. A. Pantazis, X.-Y. Chen, C. R. Landis, F. Neese, J. Chem. Theor. Comput. 2008, 4, 908-919.

(4) a) E. v. Lenthe, E. J. Baerends, J. G. Snijders, J. Chem. Phys. 1993, 99, 4597-4610; b) C. van Wüllen, J. Chem. Phys. 1998, 109, 392-399.

(5) F. Weigend, Phys. Chem. Chem. Phys. 2006, 8, 1057-1065.

(6) S. Stoll, A. Schweiger, J. Magn. Reson., 2006, 178, 42-55. 